\title{
Klaus Hartenstein
}

\section{Das erklärend-kombinatorische Wörterbuch}

im "Smysl <-> Tekst"-Modell

\section{Studien zu den lexikologischen Grundlagen der Bedeutungsexplikation und ihrer lexikographischen Verwendbarkeit}

Verlag Otto Sagner München · Berlin · Washington D.C.

Digitalisiert im Rahmen der Kooperation mit dem DFG-Projekt „Digi20“

der Bayerischen Staatsbibliothek, München. OCR-Bearbeitung und Erstellung des eBooks durch den Verlag Otto Sagner:

http://verlag.kubon-sagner.de

( $)$ bei Verlag Otto Sagner. Eine Verwertung oder Weitergabe der Texte und Abbildungen, insbesondere durch Vervielfältigung, ist ohne vorherige schriftliche Genehmigung des Verlages unzulässig. 


\title{
Slavistische Beiträge
}

\author{
BEGRÜNDET VON \\ ALOIS SCHMAUS \\ HERAUSGEGEBEN VON \\ JOHANNES HOLTHUSEN - HEINRICH KUNSTMANN \\ PETER REHDER · JOSEF SCHRENK \\ REDAKTION \\ PETER REHDER
}

Band 148

VERLAG OTTO SAGNER

MÜNCHEN 


\section{KLAUS HARTENSTEIN}

DAS EP.KLÄREND-KOMBINATORISCHE WÖRTERBUCH IM 'SMYSL $\longleftrightarrow$ TEKST'-MODELL

Studien zu den lexikologischen Grundlagen der Bedeutungsexplikation und ihrer lexikographischen Verwendbarkeit

\section{VERLAG OTTO SAGNER · MÜNCHEN 1981}


ISBN 3-87690-212-6

Copyright by Verlag Otto Sagner, München 1981

Abteilung der Firma Kubon \& Sagner, München

Druck: Alexander Grossmann

Fäustlestr. 1, D-8000 München 2

Bayerlsche Staatsbibllothok München 
Meinen herzlichen Dank möchte ich den Herren Prof.Dr. Werner Lehfeldt und Prof.Dr. Helmut Jachnow aussprechen, da sie als Gutachter am Zustandekommen der vorliegenden Arbeit maBgeblich beteiligt waren. Mein ganz besonderer Dank gilt Herrn Peter Schmidt für die wertvollen Anregungen und Ratschläge, die er mir in langen und intensiven Gesprächen gab. AuBerdem bedanke ich mich herzlich bei Frau Sigrid Neumann, Herrn Tilmann Reuther und Herrn Reinhard Köhler für ihre hilfreichen Anmerkungen.

Bei der vorliegenden Arbeit handelt es sich um die überarbeitete Fassung meiner Dissertation, deren Manuskript im Oktober 1980 fertiggestellt worden ist. 


\section{NHALTSVERZEICHNIS}

Seite

1. Zielsetzung der Arbeit

2.

DeR LeXIKOLOgische Ansatz im

"SMYSL $\leftrightarrow$ TEKST" - MODELL

2.1 Das "SMYSL $\leftrightarrow$ TEKST" - Modell und sein Lexikon

2.2 Das erklärend-kombinatorische Wörterbuch

2.2.1 Verfahren der Bedeutungsexplikation im erklärend-kombinatorischen Wörterbuch

2.2.1.1 Die semantische Sprache

2.2.1.1.1 Das Lexikon und die Syntax der semantischen Sprache

2.2.1.1.2 Der elementare Charakter der Ausdrücke der semantischen Sprache

2.2.1.1.3 Das erweiterte Lexikon der semantischen Sprache

2.2.1.1.4 Zur Heuristik der Auffindung der Ausdruicke der semantischen Sprache

2.2.1.1.5 Der ontologische Status der Ausdrücke der semantischen Sprache

2.2.1.1.6 Der künstliche Charakter der semantischen Sprache

2.2.1.1.7 Die Struktur der Ausdrücke der semantischen Sprache

2.2.1.1.8 Probleme der Synonymie und Homonymie in der semantischen Sprache

2.2.1.2 Die lexikographische Definition im er$k l a ̈ r e n d-k o m b i n a t o r i s c h e n$ Wörterbuch

2.2.1.3 Das Konzept der lexikalischen Bedeutung im erklärend-kombinatorischen Wörterbuch

2.2 .2

Die Beschreibung der kombinatorischen Eigenschaften von Lexemen im erklärendkombinatorischen Wörterbuch

2.2.2.1 Das Rektionsmodell im erklärend-kombinatorischen Wörterbuch

2.2.2.2 Die lexikalischen Funktionen im erklärend-kombinatorischen Wörterbuch 
2.2.2.3 Zum Verhältnis zwischen Bedeutung und Kombinierbarkeit der Wörter im erklärend-kombinatorischen Wörterbuch

2.3 Zur Notwendigkeit von empirischen Untersuchungen der lexikographischen Eignung des erklärend-kombinatorischen Wörterbuchs als einsprachiges Definitionslexikon

3. Festlegung des Testverfahens

4.

AUSWAHL DER INFORMANTEN

5.

KONSTRUKTION DES TESTS

5.1

Probleme der Vergleichbarkeit des erklärend-kombinatorischen Wörterbuchs im

"SMYSL $\leftrightarrow T E K S T$ " - Modell und des Definitionswörterbuchs von OZEGOV

5.1.1 Zur Vergleichbarkeit der Dekompositionsgrade konkurrierender lexikographischer Definitionen

5.2 Prinzipien der Konstruktion des Testmaterials

5.3 Auswahl des Testmaterials

6.

KONSTRUKTION DES TESTMATERIALS

8.1 Prinzipien der Auswertung der Testergebnisse

8.2 Bewertung der Reduktionsparaphrasen hinsichtlich ihrer Synonymie

8.2.1 Besprechung der Synonymierelation zwischen einigen ausgewählten Reduktionsparaphrasen unter stilistischen, morpho-syntaktischen und semantischen Aspekten 

8.3
Auswertung der Testergebnisse
245
8.3 .1
Interpretation einiger ausgewählter
Testergebnisse unter deskriptivem Aspekt
250
8.4
Zusammenfassung
254
9.
ANMERKungen
256
10.
LITERATURVERZEICHNIS
268 


\section{ZIELSETZUNG DER ARBEIT}

In der vorliegenden Arbeit gehen wir von einer doppelten zielsetzung aus. Wir wollen

1) den lexikologischen Ansatz vornehmlich unter dem Aspekt der Explikation von Wortbedeutungen darstellen und besprechen, der von den sowjetischen Linguisten JU.D. APRESJAN, A.K. ZOLKOVSKIJ und I.A. MEL'CUK im sogenannten "Model' 'Smys $l \leftrightarrow$ Tekst" (MST) entwickelt worden ist: ${ }^{1}$

2) einige der lexikographischen Beschreibungen des Russischen, die die Konstrukteure des MST in Anlehnung an ihren lexikologischen Ansatz unter der Bezeichnung "erklärendkombinatorisches Wörterbuch" ("tolkovo-kombinatornyj slovar" russkogo jazyka", EKW) vorgelegt haben, im Hinblick auf ihre praktische Tauglichkeit für Zwecke der Bedeutungsexplikation in einem noch zu erstellenden (einsprachigen russischen) Definitionslexikon empirisch untersuchen.

Die unter 1) - 2) genannten Zielsetzungen nehmen in der vorliegenden Arbeit nicht den gleichen Stellenwert ein. Die groBe Anzahl von Veröffentlichungen der Konstrukteure des MST, die ausschlieBlich die Darstellung des Modells und/oder seines Wörterbuchkonzepts zum Inhalt haben (vgl. die ausführlichen Bibliographien in BIEDERMANN 1976, 443-453, APRESJAN 1974b, 346-347 und KRYSIN 1978; 1980), läßt es uns angemessen erscheinen, den Schwerpunkt unserer Bemühungen auf die unter 2) genannte Fragestellung zu legen.

Zwar ist der Nutzwert des EKW für die praktische lexikographische Beschreibung sowie seine höhere Effektivität im Vergleich zu gängigen russischen Wörterbüchern von den Konstrukteuren des MST häufig betont und von ihren Kritikern heftig bestritten worden, unseres Wissens sind jedoch bisher keine empirischen Studien durchgeführt worden, die geeignet gewesen wären, die rein theoretisch geführte Diskussion um Vor- und Nachteile des lexikologischen Ansatzes im MST so- 
wie seiner lexikographischen Beschreibungen durch Aussagen abzusichern und gegebenenfalls zu korrigieren, die sich auf Beobachtungen des Verhaltens von Wörterbuchbenutzern stützen. In der Einschätzung des Standes der linguistischen Forschung über die Eignung des EKW für die (einsprachige) Lexikographie schlieBen wir uns daher der Beurteilung von REUTHER an:

"Betreffs der praktischen Bedeutung eines solchen Wörterbuchs [d.h. des EKW; K.H.] liegen noch keine Ergebnisse vor ..." (REUTHER 1978, 35)

Mit unserem Vorhaben stellen wir uns allerdings auch in einen Gegensatz zu demselben Autor. Dieser sieht bereits darin einen "Offnungsversuch", die praktische lexikographische Relevanz des EKW zu ermitteln, daB er zwei Wörterbuchartikel, die die Konstrukteure des MST für die russischen Lexeme kurit, und kofe erstellt haben, mit einer lexikographischen Beschreibung der entsprechenden deutschen Stichwörter kontrastiert (REUTHER 1978, 36-41). Die wichtigste GröBe, bezijglich derer Aussagen über die praktische lexikographische Bedeutung des EKW gemacht werden müssen, seine potentiellen Benutzer, werden von REUTHER nicht bericksichtigt. Angesichts dieser Forschungssituation scheint es um so notwendiger, zu versuchen, die Frage einer empirischen Beantwortung näherzubringen, inwieweit der Anspruch, den die Konstrukteure des MST dem EKW für die lexikographische Praxis zumessen, von dem Wörterbuch tatsächlich eingelöst wird.

Mit unseren empirischen Untersuchungen wollen wir auBerdem Möglichkeiten für die zukünftige Forschung in der linguistischen Semantik aufzeigen und einen Beitrag leisten, um die u.a. von FAUST konstatierte Tendenz in der gegenwärtigen Linguistik zu verstärken,

"... die Dichotomie von 'Kompetenz' und 'Performanz' aufzulösen, oder zumindest die Untersuchungen nicht mehr auf den Bereich der 'Kompetenz' im engsten Sinne zu beschränken." (FAUST 1978,365$)^{2}$ 
2. DER LEXIKOLOGISCHE ANSATZ IM "SMYSL $\leftrightarrow$ TEKST"-MODELL

\subsection{DAS "SMYSL $\leftrightarrow$ TEKST"-MODELL UND SEIN LEXIKON}

Bevor wir das EKW und den ihm zugrundeliegenden lexikologischen Ansatz näher besprechen, wollen wir das MST kurz vorstellen. Wir beschränken uns dabei auf diejenigen Aspekte des Modells, die für sein Wörterbuchkonzept wesentlich sind.

Beim MST handelt es sich um ein Stratifikationsmodell, in dem die linguistische Beschreibung auf verschiedenen, in bestimmter Abfolge einander zugeordneten Abstraktionsebenen erfolgt. Ziel der Konstrukteure des MST ist es, mit Hilfe des Modells die Sprachbeherrschung (viadenie jazykom, rečevoe povedenie) des Menschen zu beschreiben. Die Definitionen, welche die Konstrukteure des MST für den Begriff der Sprachbeherrschung bzw. der natülichen Sprache geben, sind gleichzeitig die sprachtheoretischen Grundannahmen, die dem gesamten Modell zugrundeliegen. Sie sind zwischen dem, was in der westlichen Linguistik gewöhnlich unter grammatischer und kommunikativer Kompetenz verstanden wird,angesiedelt (WELTE 1974, 256-259), fallen aber weder mit der einen noch mit der anderen Kompetenzkonzeption zusammen:

"... die Beherrschung der Bedeutung der Wörter zeigt sich beim Sprecher in der Fähigkeit, denselben Gedanken in verschiedener Weise auszudrijcken, und beim Hörer im Verstehen einer Bedeutungsgleichheit oder -ähnlichkeit äuBerlich verschiedener AuBerungen." (ZOLKOVSKIJ 1964, 4)

"Es ist unbestreitbar, daß sich die Beherrschung der Bedeutung zuallererst beim Sprecher in dem Vermögen zeigt, dasselbe in verschiedener Weise auszudrücken, und beim Hörer in dem Vermögen, die Bedeutung der Form nach verschiedener AuBerungen $z u$ identifizieren, $z u$ verstehen, daB sie dasselbe mitteilen." (ZOLKOVSKIJ/MEL'CUK 1969,6-7)

"... eine naturliche Sprache kann als eine besondere Art von (logischem) Apparat betrachtet werden, der das Verstehen einer gegebenen AuBerung, d.h. die Wahrnehmung ihrer Bedeutung(en), oder die Konstruktion von AuBerungen, die eine gegebene Bedeutung ausdrücken, gewährleistet." (MEL 'CUK 1973, 34) 
"Die naturliche Sprache ist eine besondere Art von Umformer, der gegebene Bedeutungen in die ihnen entsprechenden Texte und gegebene Texte in die ihnen entsprechenden Bedeutungen überführt." (MEL'CUK 1974b, 9)

"Es wird ... als die allgemeine Aufgabe der theoretischen Semantik angesehen, die Sprachbeherrschung zu modellieren, worunter ... die Fähigkeit des Sprechers, denselben Gedanken in verschiedener Weise auszudruicken, und die Fähigkeit des Hörers, eine semantische Identität zwischen äuBerlich verschiedenen AuBerungen festzustellen, verstanden wird. Eine solche Definition des Begriffs der Sprachbeherrschung setzt die Existenz einer besonderen, nicht direkt beobachtbaren Gedankensprache oder semantischen Sprache voraus, in der jeder Gedanke eine einzige, kanonische Ausdrucksform hat. Die obersetzung von der natuirlichen Sprache in die semantische entspricht dann dem Textverstehen (der Analyse), die Obersetzung von der semantischen Sprache in die natiurliche der Textproduktion (der Synthese)." (APRESJAN 1974b, 39-40)

Die Tatsache, daB zwischen einer gegebenen Bedeutung und der Menge aller möglichen Texte, die diese Bedeutung ausdrücken können, sowie zwischen einem gegebenen Text und der Menge aller Bedeutungen, welche dieser Text ausdrücken kann, eine ein-mehrdeutige Beziehung besteht (MEL'CUK 1974b, 31 ff.), macht es unmöglich, sowohl die Textproduktion als auch die Textrezeption im MST sozusagen in einem Synthese- bzw. Analyseschritt zu beschreiben. Die Beschreibungsergebnisse, welche man auf der linguistischen Ebene der Textbezeichneten gewonnen hat, können nicht direkt mit den Beschreibungsergebnissen korreliert werden, welche sich auf der linguistischen Ebene der Textbezeichnenden ergeben. Aus diesem Grund sind in das MST mehrere Strata der linguistischen Beschreibung aufgenommen worden. In der ersten Phase seiner Konstruktion besteht das Grammatikmodell aus vier linguistischen Ebenen (ZOLKOVSKIJ/MEL'CUK 1967, ZOLKOVSKIJ/MEL'CUK 1969; APRESJAN 1974b, 36-56, 318-344), in der zweiten Etappe seiner Entwicklung umfaBt es fünf linguistische Ebenen (MEL'CUK 1973; $1974 a, 1974 b)$. $^{3}$

Das Modell ist bisher von seinen Konstrukteuren vornehmlich im Hinblick auf die Beschreibung der Textproduktion (Textsynthese) dargestellt und besprochen worden, so daB wir uns im folgenden auch nur diese Blickrichtung zueigen machen.

Das Konzept der Sprachbeherrschung, von dem die Konstruk- 
teure des MST ausgehen, veranlaBt sie, im Grammatikmodell als erste linguistische Ebene eine Ebene der semantischen Repräsentation (smyslovaja zapis', ZOLKOVSKIJ/MEL'CUK 1967, 180 bzw. uroven' semantičeskogo predstavlenija, MEL'CUK 1974b, 32) anzusetzen, auf der die Bedeutung(en) eines gegebenen Textfragments mit Hilfe des Verfahrens der lexikalischen Dekomposition (vgl. S. $63 \mathrm{f}$.) beschrieben wird (werden). Das MST versteht sich somit - ähnlich wie die generative Semantik nordamerikanischer Prägung - als eine semantik-zentrierte Sprachtheorie:

"Der se ma $n t$ i $c h e \quad c h$ a $r k t$ e $r$ der Synthese besteht darin, daB als Ausgangsdaten für die Synthese des zu erzeugenden Texts (vychodnoj tekst) eine semantische Repräsentation, d.h. eine spezielle Repräsentation des I $n h$ a $l t s$ des Eingabetexts (vchodnoj tekst) genommen wird." (ZOLKOVSKIJ/MEL'CUK 1967, 177)

Durch die zentrale deskriptive Rolle, die im MST der Semantik als seiner tiefsten Beschreibungsebene zukommt, hebt sich das Modell deutlich von der generativen Transformationsgrammatik CHOMSKYs ab, in der die Satzgenerierung ausgehend von einer syntaktisch motivierten Tiefenstruktur erfolgt, die erst im nachhinein durch die sogenannte interpretative Semantik spezifiziert wird (IMMLER 1974, $202 \mathrm{ff.}$ ). An die Ebene der semantischen Repräsentation schließen sich die weiteren linguistischen Ebenen an, wobei zwischen den einzelnen Ebenen sogenannte Komponenten vorgesehen sind, denen die Aufgabe zukommt, beim Obergang von der Repräsentation einer gegebenen Bedeutung zur Menge aller ihr entsprechenden Texte die Beschreibungsergebnisse der einen Ebene mit denen der nächstfolgenden Ebene zu korrelieren (MEL'CUK 1974b, $35 \mathrm{ff.}$ ).

Die Intention des MST, einer gegebenen Bedeutung die Menge aller Texte zuzuordnen, welche diese Bedeutung ausdrücken können, macht es erforderlich, auf einer bestimmten stufe der Textsynthese diese Texte zu erfassen. Da es sich dabei ausschlieblich um solche Texte handeln darf, die mit dem Textfragment, dessen semantische Repräsentation als Ausgangsebene für die Synthese festgelegt worden ist, semantisch äquivalent, d.h. synonymisch sind (s.u.), spielt der Begriff der 
bedeutungsgleichen oder synonymischen Texte (ravnoznačnye, sinonimičeskie teksty) bzw. der synonymischen Mittel der natürlichen Sprache (sinonimiǒeskie sredstva estestvennogo jazyka) eine wichtige Rolle:

"... eine weitgehende und sehr fruchtbare Entwicklung erfuhren [im Laufe der Entwicklung des MST; K.H.] Oberlegungen zu den synonymischen Mitteln der naturlichen Sprache und zu den lexikalisch bedingten Ausdrucksmitteln derselben Bedeutung ..." (APRESJAN 1974b, 43)

MEL'CUK finrt den Begriff der Bedeutungsgleichheit (von Texten) axiomatisch ein und benutzt ihn als Ausgangsbegriff für die Definition des Begriffs der Bedeutung (smysz):

"Der Begriff der Bedeutungsgleichheit ist für uns formal undefinierbar. Inhaltich können Texte bedeutungsgleich genannt werden, wenn Sprecher der gegebenen Sprache behaupten, daB 'diese Texte dasselbe bedeuten', daB 'in innen dieselbe Information enthalten ist. Einfach gesagt, der Begriff der Bedeutungsgleichheit von Texten wird als intuitiv evident betrachtet." (MEL'CUK 1974b, 10)

"Bedeutung wollen wir als Invariante einer Menge bedeutungsgleicher Texte verstehen, wobei wir deren Bedeutungsgleichheit als intuitiv evident fur einen 'native speaker' betrachten." (MEL'CUK 1973, 34)

Unter den Begriff der Bedeutungsgleichheit (oder Synonymie) von Texten subsumieren die Konstrukteure des MST nicht nur Texte, zwischen denen die Relation der Bedeutungsäquivalenz (oder der genauen Synonymie) besteht, sondern auch solche, die zueinander im Verhältnis der Quasisynonymie stehen. Aus der Menge der quasisynonymischen Texte heben die Konstrukteure des MST solche Texte besonders hervor, zwischen denen die Relation der Bedeutungsimplikation besteht:

"Die Beherrschung der Bedeutungen der Wörter zeigt sich auch in der Fähigkeit zum semantischen SchluB (sposobnost' $k$ semantičskomu vyvodu), d.h. in dem Vermögen, in Anlehnung an ein bereits aufgetretenes Textfragment Hypothesen iber den möglichen Inhalt seiner nachfolgenden Teile zu konstruieren." (APRESJAN 1967,8)

"... die Sprachbeherrschung zeigt sich in der Fähigkeit zum semantischen SchluB. Fir jeden Sprecher des Russischen setzt die Bedeutung des Satzes: Ivan poterjal knigu die Be- 
deutung des Satzes: Kniga u Ivana propala voraus, nicht aber umgekehrt. In der gleichen Relation der semantischen Folgerung steht die Bedeutung der Sätze: Ivan prinës knigu sjuda $\rightarrow$ Kniga nachoditsja zdes', Ivan prinosil knigu sjuda $\rightarrow$ Kniga nachodilas' zdes', Ivan unës knigu otsjuda $\rightarrow$ Kniga nachodilas' zdes'; vgl. auch die Folgeimplikation in dem Satztripel: Dozd'

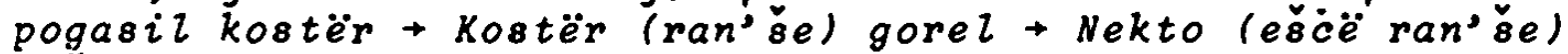
zažeg kostër." (APRESJAN 1968, 35; vgl. für weitere Beispiele auch MEL' CUK 1974b, 152-159)

Da die Konstrukteure des MST die Bedeutungsgleichheit von Texten als intuitiv evident betrachten, können sie keine Kriterien angeben, mit deren Hilfe bedeutungsgleiche Texte in strengsynonymische und quasisynonymische Texte zu unterscheiden sind. Bei der Unterscheidung beider Klassen von Texten gehen die Konstrukteure des MST daher ebenfalls intuitiv vor. 4

Um die Menge aller Texte, die mit einer gegebenen semantischen Repräsentation bedeutungsgleich sind, beschreiben zu können, sehen die Konstrukteure des MST ein System synonymischer Paraphrasen (sistema sinonimičeskich perifraz, sistema sinonimičeskogo preobrazovanija, sistema sinonimičeskogo perifrazirovanija) vor:

"... für jede Ausgangsrepräsentation einer Bedeutung muB der Algorithmus möglichst viele (im Idealfall sämtliche) verschiedenen Varianten ihres Ausdrucks in der zu erzeugenden Sprache (vychodnoj jazyk) konstruieren." (ZOLKOVSKIJ/MEL'CUK 1967, 177-178)

"Da die Abbildung einer Bedeutung in ihre verschiedenen Ausdrucksweisen als wesentlicher Aspekt der sprachlichen Beherrschung der Bedeutung anzusehen ist, nimmt die Modellierung der Synonymie, d.h. die Konstruktion von funktionalen (rabotajuščie) Paraphrasesystemen in der modernen Semantik einen erstrangigen Platz ein." (ZOLKOVSKIJ/MEL'CUK 1969,7)

Das System synonymischer Paraphrasen operiert uber einer bzw. mehreren lexikalisch-syntaktischen Basisstruktur(en), die der semantischen Repräsentation einer gegebenen Bedeutung im MST auf der unmittelbar auf die semantische Repräsentationsebene folgenden tiefensyntaktischen Beschreibungsebene zugeordnet wird bzw. werden. 5 Es hat die Aufgabe, fïr die lexikalischsyntaktischen Basisstruktur(en) sämtliche synonymischen lexikalisch-syntaktischen Strukturen anzugeben: 
"Das Paraphrasesystem - der Hauptbestandteil der semantischen Synthese - ist eine Menge von Regeln, die den Obergang von der lexikalisch-syntaktischen Struktur eines beliebigen russischen Satzes ... zu allen anderen ihr synonymischen lexikalisch-syntaktischen strukturen gewährleistet." (ZOLKOVSKIJ/MEL'CUK 1967, 181)

Um die Menge aller untereinander synonymischen lexikalischsyntaktischen Strukturen, die mit der Repräsentation einer gegebenen Bedeutung semantisch im engen oder weiten Sinne äquivalent sind (s.o.), beschreiben zu können, sind im system synonymischer Paraphrasen lexikalische und syntaktische Umformungsregeln vorgesehen:

"Die Umformungen einer tiefensyntaktischen Struktur [d.h. einer lexikalisch-syntaktischen Struktur; K.H.] in eine andere bestehen in der synonymischen Substitution entweder ihrer Lexik... oder ihrer syntaktischen Verknupfungen ... oder - und das ist der häufigste Fall - in beidem zugleich. Dementsprechend zerfallen die Umformungsregeln in zwei Klassen: lexikalische und syntaktische." (MEL'CUK 1974b, 149)

Zur Speicherung der linguistischen Informationen, welche erforderlich sind, um mit den Umformungsregeln des Systems synonymischer Paraphrasen operieren zu können, haben die konstrukteure des MST ein spezielles Lexikon entworfen. Dieses Wörterbuch, das EKW, ist ein wesentlicher Bestandteil des Grammatikmodells. Es fixiert bezüglich eines bestimmten Lexems, das als Stichwort ausgewählt wird, in Form von Wörterbuchartikeln u.a. sämtliche Angaben, die für die Arbeit des Paraphrasesystems benötigt werden:

"Das 'Smys $\leftarrow$ Tekst'-Modell verwendet in ganz essentieller Weise eine grobe Menge von Informationen uber jedes Wort der betrachteten Sprache ... Diese Angaben können in einem speziellen Lexikon gemacht werden, welches wir als das e $r$ l $\ddot{a} r$ e $n d-k 0 m b i n a t o r i s c h e$ w $t e r b u c h$ (EKW) bezeichnen und welches im hier beschriebenen Modell eine wichtige Stellung einnimmt... Im Idealfall muB ein Wörterbuchartikel eines solchen Lexikons sämtliche Charakteristika zur Flexion, Wortbildung, Syntax, Semantik und Stilistik eines Stichworts $C_{0}$ (zaglavnoe slovo, kijucevoe slovol enthalten; auBerdem ist es erforderlich, für jedes $C_{0}$ alle Wörter (oder Wortverbindungen) anzugeben, die mit inm bedeutungsmäBig auf eine bestimmte Art verbunden sind [d.h. seine lexikalischen Funktionen; K.H.; vgl. S. $97 \mathrm{ff.]}$." (MEL'CUK 1974b, 78) 
Im folgenden wollen wir das EKW unter bestimmten lexikologischen und lexikographischen Aspekten näher besprechen.

\subsection{Das erklärend-kombinatorische Wörterbuch 6}

Zweck und Standort des EKW im MST weisen das Wörterbuch zunächst als ein rein linguistisches Lexikon aus, da es Teil eines Grammatikmodells ist. Die Charakterisierung des EKW kann jedoch in dieser Form nicht aufrechterhalten werden. In den Veröffentlichungen der Konstrukteure des MST und ihrer Mitarbeiter läßt sich die durchgängige Tendenz beobachten, die lexikologischen Prinzipien des Wörterbuchs und die entsprechenden lexikographischen Beschreibungen von Teilen des russischen Wortschatzes mit dem Anspruch vorzustellen, über eine bessere Alternative zu bereits vorhandenen russischen Definitionslexika sowohl in theoretischer als auch in praktischer Hinsicht 2u verfügen (APRESJAN 1966; 1967; 1968; 1969a; 1969c; 1974b; APRESJAN/ZOLKOVSKIJ/MEL'CUK 1969b; 1973; MEL'CUK 1970; 1974b). Dabei gehen die Konstrukteure des MST von der stillschweigenden Voraussetzung aus, daß es möglich sei, das Lexikon aus seinem Modellbezug herauszulösen und unter bestimmten Modifikationen (s.u.) für andere Zwecke als diejenigen, denen es ursprünglich und primär genügen sollte, einzusetzen.

Die Konstrukteure des MST meinen, mit dem EKW ein Wörterbuch eines völlig neuartigen Typs von hoher Effektivität für die lexikographische Praxis vorzulegen. Dieser Anspruch zeigt sich deutlich in ihrem Vorgehen bei der Darlegung des EKW, welches in den meisten Fällen oppositiv zur Beschreibungspraxis gängiger russischer Definitionslexika erfolgt. Der Praxisbezug, den die Konstrukteure des MST für das EKW postulieren, wird außerdem dadurch unterstrichen, daß sie für Zwecke der Explikation von Wortbedeutungen im EKW eine spezielle künstliche Definitionssprache, die sogenannte semantische Sprache (SemS) entwickeln, welche sich strukturell von der Metasprache, die im MST zur semantischen Beschreibung auf der Ebene der semantischen Repräsentation verwen- 
det wird, erheblich unterscheidet (s.u.).

Einen weiteren Beleg für den Nachdruck, mit dem die Konstrukteure des MST den nicht ausschlieBlich theoretischlinguistischen oder modellbezogenen Charakter des EKW betonen, stellt die hohe Anzahl von lexikographischen Definitionen russischer Lexeme mit Hilfe der SemS des EKW dar, die sich in verschiedenen Arbeiten zu Problemen des EKW (s.o.) und insbesondere unter der Bezeichnung "Materialy $k$ tolkovo-kombinatornomu slovarju russkogo jazyka" in Publikationen der Serie "Predvaritel'nye publikacii problemnoj gruppy po eksperimental'noj i prikladnoj lingvistike" finden." Die Notwendigkeit, zwischen einem modellbezogenen EKW und einem EKW, das lexikographische Bedirfnisse von Sprechern befriedigen soll, zu unterscheiden, wird von den konstrukteuren des MST lediglich in einer der uns verfügbaren Arbeiten (in einer Fußnote!) erwogen:

"Man sollte vielleicht von zwei Typen Definitionslexika sprechen - solche fïr Sprecher (vgl. die Schulgrammatik) und solche fiir Linguisten (vgl. die theoretische Grammatik), der erste Typ müBte dennoch immer die Ergebnisse des zweiten verwenden." (APRESJAN 1969a, 11, Anm. 2)

Es ist zu vermuten, daB die Konstrukteure des MST aufgrund des engen Zusammenhangs, den sie zwischen beiden Wörterbuchtypen konstatieren, in ihren einschlägigen Publikationen darauf verzichten, anzugeben, welche Variante des EKW - die modellbezogene, theoretisch-linguistische oder die praktisch-lexikographische - sie beschreiben wollen.

Im folgenden wollen wir das EKW vornehmlich im Hinblick auf seine Explikationspraxis unter lexikologischen und lexikographischen Aspekten darstellen und besprechen. In Anlehnung an die eher praxisorientierte Zweckbestimmung, die die Konstrukteure des MST für das EKW vorsehen, betrachten wir das Wörterbuch als einen besonderen Typ von Lexikon, das auch mit dem Anspruch entwickelt worden ist, praktische lexikographische Bedirfnisse von Sprechern als seinen potentiellen Benutzern zu befriedigen. 
2.2.1 Verfahren der Bedeutungsexplikation im erklärendkombinatorischen Wörterbuch

\subsubsection{Die semantische Sprache}

Zur Explikation von Wortbedeutungen (tolkovanie slova, leksikografičeskoe opredelenie slova) bedient sich das EKW der Sems als einer speziell für lexikographische Zwecke entwickelten Definitionssprache. Für lexikographische Definitionen, die mit Hilfe der SemS erfolgen, schlägt APRESJAN eine formale und eine wortsprachliche Notation vor. Anstelle des Formalismus, der von APRESJAN nur an wenigen Beispielen von Bedeutungsexplikationen erläutert wird, verwenden die Konstrukteure des MST in den lexikographischen Definitionen des EKW ausschlieBlich die wortsprachliche Notationsvariante der SemS. Dieses Vorgehen begründet APRESJAN damit, daß Bedeutungsexplikationen, die in der wortsprachlichen Notationsvariante der Sems abgefaßt sind, besser uberschaubar und eher zugänglich seien (vgl. auch MEL'CUK 1974b, 111), was für Definitionen, die praktische lexikographische Bedürnisse befriedigen sollen, zweifellos eine wesentliche Eigenschaft ist. Gleichzeitig unterstreicht er, daß zwischen der formalen und der wortsprachlichen Notationsvariante der Sems absolute Aquivalenz bestehe:

"Es versteht sich von selbst, daß derartige [d.h. formale; K.H.] Bedeutungsexplikationen hauptsächlich den Erfordernissen einer formalen Theorie dienen. Sie können jedoch leicht und absolut eindeutig in eine wortsprachliche formulierung übersetzt werden, die auch für gewöhnliche Definitionswörterbücher geeignet ist." (APRESJAN 1969c, 423)

"Im folgenden werden wir wortsprachliche formulierungen (slovesnye formulirovki) verwenden, weil sie leichter erfaßt werden können und weniger Raum einnehmen. Wir wollen jedoch betonen, daß eine jede derartige Formulierung mit Hilfe einfacher Regeln und von Information, welche unmittelbar in den Wörterbuchartikeln des von uns vorausgesetzten Lexikons [der SemS; K.H.] enthalten ist, in eine völlig korrekte und strenge Form der Bedeutungsrepräsentation durch einen Baumgraphen expandiert werden kann." (APRESJAN, $1974 b, 78-79$ ) 
der SemS des EKW als einer Definitionssprache für lexikographische Zwecke und der semantischen Sprache besteht, welche in der zweiten Fassung des MST auf der Ebene der semantischen Repräsentation bei der Beschreibung der Bedeutung eines gegebenen Textes Verwendung findet:

"Die semantische Sprache im strengen Sinne des Wortes ist eine Sprache semantischer Graphen (MEL'CUK 1974b). Wir schlagen lediglich eine erste Annäherung an diese vor - eine quasisemantische Sprache von Dependenzbäumen, die in erster Linie zur Formalisierung gewöhnlicher lexikographischer Definitionen bestimmt ist ... Im folgenden wird diese quasisemantische Sprache der Küze wegen einfach semantisch genannt." (APRESJAN 1974b, 69, Anm. 3)

In der Tat lassen sich zwischen der SemS des EKW und der Metasprache, die im MST auf der Ebene der semantischen Repräsentation verwendet wird, erhebliche Unterschiede ausmachen. Bei letzterer handelt es sich um semantische Netze oder gerichtete Graphen, in denen die semantischen Komponenten, welche für die Beschreibung der Bedeutung eines Textes angesetzt werden, durch inhaltich und syntaktisch nicht näher spezifizierte Relationen mit Hilfe von Pfeilen miteinander verknipft werden. Steht eine gegebene Bedeutungskomponente zu mehreren anderen Bedeutungskomponenten in einer wie auch immer gearteten Relation, so werden diese Relationen lediglich durch Ziffern als verschieden ausgewiesen. Die Anzahl der Relationen, die eine gegebene Bedeutungskomponente mit anderen eingehen kann, ist in der Regel auf zwei begrenzt (MEL'CUK 1974b, $62 \mathrm{ff.}$ ).

Im folgenden werden wir auf die SemS des EKW näher eingehen. Dabei werden die Unterschiede, die sie im Vergleich zu der oben skizzierten Beschreibungssprache der Ebene der semantischen Repräsentation des MST aufweist, deutlich hervortreten. 
2.2.1.1.1 Das Lexikon und die Syntax der semantischen Sprache

Die SemS des EKW verfügt über ein eigenes Lexikon, in dem ihre "Wörter" vermerkt sind, und über eine eigene Dependenzsyntax, die angibt, auf welche Weise diese "Wörter" miteinander verknüpft werden können. Die Konstrukteure des MST haben bisher absichtlich noch kein endgïltiges, geschlossenes Inventar von Ausdrucksmitteln der SemS des EKW angegeben:

"Die Listen [der Ausdrücke der SemS; K.H.] sind nicht erschöpfend; sie geben ausschlieBlich die elementaren Bedeutungen wieder, die bisher als Ergebnisse einer empirischen Untersuchung gefunden worden sind. Es ist zu erwarten, daB sie im Laufe der weiteren Arbeit aufgefiult werden."

(APRESJAN 1969c, 416-417)

"Zur Zeit ist das vorgeschlagene Dependenzsystem [d.h. die Syntax der SemS; K.H.J genauso wie das Lexikon der semantischen Sprache weder fixiert noch geschlossen; es kann sich bei Veränderungen im Bestand der elementaren Bedeutungen vergröBern oder verkleinern." (APRESJAN 1969C, 420)

"Sie [d.h. die SemS des EKW; K.H.] wird langsam, aber folgerichtig ausgebaut. In der ersten Zeit ist es besser, sich mit einer beschränkten semantischen Sprache zu begnügen, von der uns alle Eigenschaften bekannt sind, als sie ständig durch Elemente mit undefinierten Eigenschaften aufzufiullen. Der Wert solcher Elemente ... ist oft zweifelhaft." (APRESJAN 1971 a, 27) 8

"Dem Autor ist nicht bekannt, wie groB (auch nur annähernd) die Anzahl der Seme ist, die für die Beschreibung der signifiés der naturlichen Sprache erforderlich sind.

Forscher, die auf diesem Gebiet arbeiten, haben GröBenordnungen von dreihundert bis anderthalb oder zweitausend elementaren Bedeutungen genannt ... Derartige Zahlen sind jedoch gewöhnlich nicht vergleichbar, da verschiedene Forscher unterschiedliche Tiefen der Bedeutungsdekomposition und, was am wichtigsten ist, unterschiedliche Umfänge des Wortschatzes, der in Termini von Semen beschrieben werden soll, im Sinn haben. (Die einen wollen nur die abstrakte Lexik, andere nur die konkrete lgemeint sind wohl lexikalische Einheiten mit abstrakten bzw. konkreten Referenten; K.H.l, wieder andere sowohl die eine als auch die andere beschreiben)." (MEL'CUK 1974b, 58)

In verschiedenen Veröffentlichungen schlagen die Konstrukteure des MST für die SemS des EKW unterschiedliche Bestände an "lexikalischen" und syntaktischen Ausdrucksmitteln 
vor. In der ersten Entwicklungsstufe der SemS des EKW, die in APRESJAN 1969 c vorgestellt wird, umfaBt das Lexikon der Sems sechs verschiedene klassen von Ausdruicken:

(1) Bezeichnungen für elementare Prädikate wie beispielsweise 'vosprinimat', 'dvigat'sja', 'kauzirovat', 'cuvstvovat' ' u. à.;

(2) Bezeichnungen für elementare Gegenstände, unter denen APRESJAN nicht nur Eigennamen, sondern auch Prädikate wie beispielsweise 'vremja', 'mesto', 'sostojanie', 'cel"' u.a. versteht;

(3) Bezeichnungen für elementare Deskriptoren oder Klassifikatoren wie beispielsweise 'bol'se', 'neobchodimyj', 'neposredstvennyj', 'toždestvennyj' u.ä.;

(4) die Junktoren (logičeskie sujazki) ' $i$ ', ' $i i_{i}$ ' und 'ne';

(5) den Allquantor 'vsjakij' und den Existenzquantor 'imeetsja';

(6) Gegenstandsvariable wie beispielsweise ' $A$ ', ' $B$ ', ' $C$ ' u. ä. (APRESJAN 1969c, 416)

Die Syntax der SemS des EKW umfaBt insgesamt sieben verschiedene Dependenzrelationen. Sechs dieser Relationen bezeichnen Dependenzen, die sich unmittelbar aus den Valenzen herleiten lassen, welche die Konstrukteure des MST den Bezeichnungen für elementare Prädikate, d.h. den "Wörtern" der Klasse (1) zuweisen. Bei diesen Dependenzen, die inhaltlich interpretiert und in Form sogenannter indizierter Pfeile notiert werden, handelt es sich um eine Subjektvalenz $\stackrel{S u b}{\longrightarrow}$, eine Objektvalenz $\stackrel{O b j}{\longrightarrow}$, eine Lokalvalenz $\stackrel{\text { Loc }}{\longrightarrow}$, eine Endpunktvalenz $\stackrel{A d}{\rightarrow}$, eine Anfangspunktvalenz $\stackrel{A b}{\rightarrow}$ und eine Valenz, die die Strecke einer Bewegung (oblast', maršrut) bezeichnet, Itin. Als siebte Dependenzrelation, die nicht direkt aus Valenzeigenschaften bestimmter "Wörter" der Sems des EKW herleitbar ist, wird eine Attributionsrelation $\stackrel{A t t r}{\longrightarrow}$ angesetzt (APRESJAN 1969c, 419). Der gegenwärtige Entwicklungsstand der SemS des EKW, der in APRESJAN 1974 b besprochen wird, zeichnet sich dadurch aus, daB das Lexikon der SemS im Vergleich zu APRESJAN 1969c auf vier Klassen von 
"Wörtern" reduziert worden ist. Es werden die folgenden Klassen von Ausdrücken angesetzt:

(1) Bezeichnungen für elementare Prädikate;

(2) Bezeichnungen für elementare Gegenstände;

(3) die Junktoren ' $i$ ', ' $i l i$ ' und ' $n e$ ';

(4) Gegenstandsvariable (APRESJAN 1974b, 73-74).

Die Reduktion des Lexikons der SemS wurde möglich, da APRESJAN nun die Bezeichnungen für elementare Deskriptoren und den Existenzquantor, der in APRESJAN $1969 \mathrm{C}$ durch 'imeetsja', in APRESJAN $1974 \mathrm{~b}$ jedoch durch 'suščestvuet' etikettiert wird (hierzu auch S. $43 \mathrm{ff}$.), als einfache elementare Prädikate behandelt und den Allquantor als komplexes Prädikat aus der bekannten Verknüpfung des Existenzquantors mit der Negation herleitet: 'Vsjakij $x$ dejstvuet.' = 'Ne suščstvuet $x$-a, kotoryj by ne dojstvoval.

Auch hat die Syntax der Sems in ihrem Inventar Veränderungen erfahren. Sie umfaßt insgesamt acht Dependenzrelationen. Im Vergleich zu APRESJAN 1969c enthält sie jetzt neben der Attributions-, der Subjekt-, der Objekt- und der Lokalvalenz eine Kontragentralenz 'bol'še' Contrag ' $B$ ', eine Inhaltsvalenz 'chotet' ' Content ' $B$ ', eine Temporalvalenz 'načinat'sja' TemR ' $B$ ' und eine Quantitätsvalenz 'bol'še' Quant ' $B$ ' (APRESJAN 1974b, 78, 130).

Der veränderte Bestand, den die Syntax der SemS des EKW in ihrem gegenwärtigen Entwicklungsstand im Vergleich zu APRESJAN 1969c aufweist, ist darauf zurückzufïhren, daB APRESJAN jetzt die Valenzen ' $A d$ ', ' $A b^{\prime}$ ' und ' $I$ tin' als inhaltich komplex ansieht und sie aus der Lokalvalenz 'Loc', die er als elementar ansetzt, ableitet (APRESJAN 1974b, 127-130). In gleicher Weise betrachtet er die im Vergleich zu APRESJAN 1969 C neu eingeführten Valenzen 'Contrag' 'Content', 'Temp' und 'Quant' als elementar. Die unterschiedlichen Aufgliederungen, die der Bestand an "lexikalischen" und syntaktischen Ausdrucksmitteln der SemS des EKW im Laufe seiner Ausarbeitung erfahren hat, sowie der Verzicht der Konstrukteure des MST darauf, ein geschlossenes Inventar an entsprechenden Ausdrucksmitteln zu bestimmen, sollte nicht als ein prinzipieller Mangel der semantischen 
Beschreibung im EKW angesehen werden. Wir teilen daher nicht die Ansicht von ULUCHANOV, der dem EKW gerade diesen Vorwurf macht:

"In Arbeiten, die der semantischen Deskription gewidmet sind, ist eine Sprache zur Beschreibung von Wortbedeutungen nicht ausgearbeitet. Die semantische Metasprache, iber die in der letzten Zeit eine Diskussion gefihrt wird, ist nicht erstellt worden." (ULUCHANOV 1977, 17)

Angesichts des breiten Raums, den Ausführungen zum Inventar der SemS des EKW und Auflistungen ihrer Ausdrucksmittel in verschiedenen Veröffentlichungen der Konstrukteure des MST einnehmen ( $v g$ l. insbesondere APRESJAN 1968, 1969c, 1974b), ist die Behauptung, daß die Sems uberhaupt nicht existiere, unhaltbar. Wir schließen uns vielmehr der Einschätzung der Konstrukteure des MST an und werten den unfertigen Charakter der SemS des EKW als ein Indiz dafür, daB es sich um ein Beschreibungsinstrumentarium handelt, welches nur schrittweise ausgebaut werden kann (s.0.).

Die Trennung in Lexikon und Syntax der Sems, die wir bei der Besprechung ihres Inventars vorgenommen haben, diente nur einer klareren Darstellung der beiden Bereiche. Die Konstrukteure des MST nehmen die Syntax der SemS fast vollständig in ihr Lexikon auf, da sie mit Ausnahme der Attributionsrelation alle anderen Relationen, die die Syntax der Sems umfaBt, aus den Valenzen herleiten, die sie den elementaren Prädikaten zuweisen. Die Bezeichnungen für elementare Prädikate oder die "Wörter" der Klasse (1) nehmen daher im Lexikon der Sems eine zentrale Rolle ein.

Der Hinweis APRESJANS, daB es erforderlich sei, im Lexikon der sems

"... jede elementare Bedeutung der ersten Klasse mit Angaben zur Anzahl ihrer Leerstellen, ihres semantischen Gehalts und zur kleinstmöglichen Ordnung des Prädikats zu versehen, das jede dieser Leerstellen einnehmen kann" (APRESJAN 1969c, 418 ),

ist dadurch bedingt, daß die elementaren Prädikate sich durch eben diese Eigenschaften unterscheiden können.

Die Konstrukteure des MST machen keine Angaben zur höchstmög- 
lichen Anzahl von Leerstellen, die sie einem elementaren Prädikat zuweisen wollen. Aus den Beispielen, die sie für elementare Prädikate mit unterschiedlicher Anzahl von Leerstellen anführen, geht hervor, daß diese Prädikate wahrscheinlich über nicht mehr als vier Leerstellen verfügen dürfen. Beispielsweise eröffnet das Prädikat 'suščestvovat' ' eine Leerstelle ('A suščestvuet'), das Prädikat 'vključat'sja' zwei Leerstellen ('A vključaetsja $v B^{\prime}$ ) das Prädikat 'bol'še' drei Leerstellen (' $A$ bol'se $B-a n a C$ ') und das Prädikat 'duigat'sja' vier Leerstellen ('A dvigaetsja $i z B-a \cup C$ po D-u') (APRESJAN 1974b, 76; 1969c, 417).

Der semantische Gehalt der Leerstellen kann bei elementaren Prädikaten mit gleicher Anzahl von Leerstellen verschieden sein. Beispielsweise unterscheiden sich die beiden zweiwertigen Prädikate 'imet' ' und 'nachodit'sja' hinsichtlich des semantischen Gehalts ihrer zweiten Leerstelle, die von den Konstrukteuren des MST bei 'imet' ' als eine Objektvalenz ('imet' 'Obj ' $B^{\prime}$ ), bei 'nachodit'sja' als eine Lokalvalenz ('nachodit'sja' $\stackrel{\text { Loc }}{\longrightarrow} B^{\prime}$ ') inhaltlich interpretiert wird.

Die Konstrukteure des MST weisen ausdrücklich darauf hin, daB sie über keine Auffindungsprozedur verfügen, um die An$z a h l$ der Leerstellen eines elementaren Prädikats und deren entsprechenden semantischen Gehalt zu ermitteln:

"Da ein elementares Prädikat undefiniert eingeführt wird, können weder die Anzahl der Leerstellen, die ihm zugeschrieben werden, noch ihr semantischer Gehalt objektiv begründet werden." (APRESJAN 1969c, 418; 1974b, 76)

Die Spezifizierung der syntaktischen und semantischen Eigenschaften eines elementaren Prädikats ist daher völlig von der Intuition des Analysators abhängig. Aus diesem Grunde durfte es beispielsweise für das Prädikat 'vosprinimat' ', das die Konstrukteure des MST ins Lexikon der Sems aufnehmen (APRESJAN 1969c, 416), unmöglich sein, anders als introspektiv zu entscheiden, ob der semantische Gehalt seiner zweiten Leerstelle als Inhaltsvalenz ('vosprinimat" Content ' $B^{\prime}$ ) oder als objektvalenz ('vosprinimat' ' $\underline{O b_{j}}$ ' $B$ ') interpretiert werden kann. 
Die Ordnung (porjadok) eines elementaren Prädikats richtet sich nach der Klassenzugehörigkeit der Ausdrücke der Sems, die seine Leerstellen besetzen können. Kann bei einem gegebenen Prädikat auch nur eine Leerstelle durch ein anderes (elementares) Prädikat besetzt werden, dessen Argumentstellen ausschließlich Bezeichnungen für Gegenstände einnehmen können, so handelt es sich bei ersterem um ein Prädikat zweiter, bei letzterem um ein Prädikat erster Ordnung. Ein solches Prädikat ist beispielsweise 'kauzirovat' ' in der Explikation von vešats
(APRESJAN 1974b, 78): ${ }^{9}$

$A$ versaet $B$ na $C=$

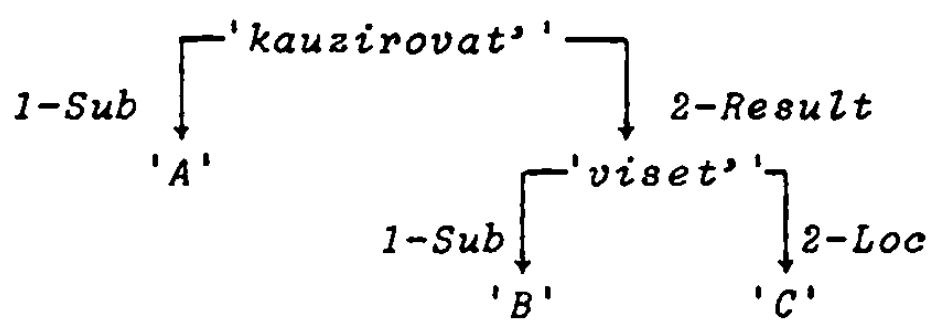

Das elementare Prädikat 'viset' ' ist dagegen ein Prädikat erster Ordnung, da keine seiner Leerstellen durch andere Ausdrücke der Sems als Bezeichnungen für elementare Gegenstände bzw. Gegenstandsvariable eingenommen werden können (APRESJAN 1969c, 418; 1974b, 76).

2.2.1.1.2 Der elementare Charakter der Ausdrücke der semantischen Sprache

Die Ausdrücke der SemS werden von den Konstrukteuren des MST als elementare Bedeutungen (élementarnye značenija, è lementarnye smysly) oder als Bedeutungsatome (smyslovye atomy) bezeichnet. Ausschlaggebend für den elementaren oder a tomaren Charakter der "Wörter" der SemS ist ihrer Ansicht nach ausschließlich die Tatsache, daß sie undefiniert (nicht undefinierbar!) in das Lexikon der Sems übernommen werden (APRESJAN 1969a, 14; 1969c, 418; 1974b, 76). Die Elementarität eines Ausdrucks der SemS steht daher in direkter Abhängigkeit von der Menge der "Wörter", die der je- 
weilige Forscher in ihr Lexikon aufnimmt:

"Das Wort 'elementar' muß hinsichtlich der Seme (Sem, d.h. elementare Bedeutung) als ausgesprochen relativ verstanden werden: 'elementar' bedeutet 'im Rahmen des gegebenen Beschreibungssystems nicht zerlegbar'. Das, was für eine Beschreibung ein Sem ist, kann für eine andere eine Konstruktion aus mehreren Semen sein. So kann der eine Forscher 'znat' als Sem betrachten, der andere dagegen 'znat' in 'obladat' "und 'informacija' ('znat' ' = 'obladat' informacie $j^{\prime}$ ) dekomponieren, indem er 'informacija' und 'obladat. " als seme ansieht, usw. Wichtig ist nur, in wesentlichen Fällen den relativen Charakter der Seme zu vereinbaren. Wir wollen uns an dieser Stelle jeglicher Kommentare zu Möglichkeiten enthalten, 'absolut' elementare Seme zu ermitteln." (MEL'CUK 1974b, 58)

APRESJAN demonstriert anhand einiger Ausdrücke der SemS des $E K W$, daB sie mit Hilfe anderer, ebenfalls in ihrem Lexikon enthaltener "Wörter" definiert werden könnten und daher im Rahmen dieses Beschreibungsinstrumentariums nur relative Elementarität beanspruchen können:

"... wir haben es uns nicht zum ziel gesetzt, als elementare Bedeutungen nur wirklich elementare Begriffe $2 u$ verwenden. Einige der angeführten elementaren Bedeutungen sind in Wirklichkeit nicht elementar. So gilt 'situacija' = 'Predmety $i$ otnosenija mezdu nim $i^{\prime}$ und 'cel' $X-a$ ' $^{\prime}$ ' 'Ylatel'naja dlja $x$-a situacija, kotoruju $x$ možet kauzirovat" ..." (APRESJAN $1969 c, 417$ )

Als nicht elementar kann auch das Prädikat 'načinat'sja' angesehen werden, da es durch andere Ausdrücke der Sems definiert werden könnte; vgl. die Zerlegungen (1) - (3):

(1) 'X načalsja v $T_{j}^{\prime}=$ ' $V$ moment $T_{i} \times$ ne suščestvoval, $i$ $v$ moment $T_{j}$ suščestvoval, $i T_{j}$ pož̃e $T_{i}$.

(2) 'moment'

= 'Točka vremeni'

(3) ' $T_{j}$ pozže $T_{i}$ '

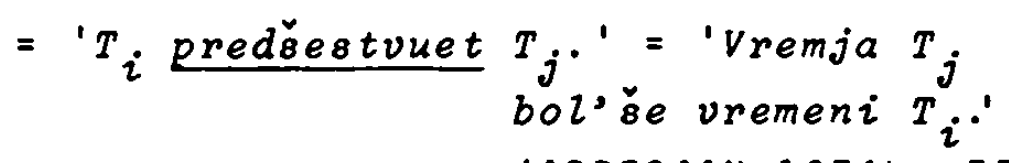
(APRESJAN 1974b, 75)

Betrachten die Konstrukteure des MST Ausdrücke der SemS wie beispielsweise 'situacija', 'cel' und 'načinat'sja' dennoch als im oben genannten Sinne elementar, so lassen sie sich ausschließlich von Oberlegungen zur Einfachheit und Okonomie 
der semantischen Beschreibung leiten (APRESJAN 1969c, 417 ; $1974 \mathrm{~b}, 64)$.

2.2.1.1.3 Das erweiterte Lexikon der semantischen Sprache

Die Konstrukteure des MST berufen sich auf dieselben Erwägungen, um das Lexikon der Sems durch ein erweitertes Lexikon (rasširënnyj slovar') zu ergänzen. Die Notwendigkeit für ein erweitertes Lexikon der Sems ergibt sich aus dem Bestreben, Bedeutungsexplikationen, die mit Hilfe der SemS vorgenommen werden, möglichst ökonomisch und überschaubar zu halten.

Kann die Forderung, Bedeutungen möglichst ökonomisch zu explizieren, noch als ein rein linguistisches Beschreibungsprinzip begriindet werden, so steht das Postulat nach oberschaubarkeit und Einfachheit der lexikographischen Definitionen, die in der SemS des EKW abgefaBt werden, in direktem Zusammenhang mit dem Anspruch des Wörterbuchs, praktische lexikographische Bediurfnisse seiner potentiellen Benutzer zu befriedigen. Sollen die lexikographischen Definitionen des EKW ihren potentiellen Adressaten Auskunft über die Bedeutung eines Stichworts geben, missen sie für diese zugänglich, d.h. verstehbar sein. Zu hohe Komplexität durfte diese wesentliche Eigenschaft der lexikographischen Definitionen des EKW zweifellos stark beeinträchtigen. Semantische Beschreibungen, in denen ausschlieBlich elementare Ausdrücke zur Verwendung gelangen, werden daher kaum uber die geforderte Einfachheit verfügen. 10

Das Prinzip des erweiterten Lexikons wird bereits 1964 von ZOLKOVSKIJ kurz angesprochen, ohne allerdings als solches bezeichnet zu werden:

"Wörter, welche in diesen Einheiten [d.h. in elementaren semantischen Einheiten; K.H.] definiert worden sind, können weiterhin selbst als Instrumente eingesetzt werden, um komplexe Wörter zu beschreiben ..." (ZOLKOVSKIJ 1964, 7-8)

In ähnlicher Weise äuBert sich auch WEINREICH, der für die kontrollierte Reduktion einer semantischen Metasprache, die 
in lexikographischen Definitionen verwendet werden soll, das folgende Stratifikationsverfahren vorschlägt:

"... man kann das Vokabular einer Sprache [d.h. einer semantischen Metasprache; K.H.l in einer Weise betrachten, daB es aus den folgenden verschiedenen Ebenen besteht:

Ebene 0: Ausdrücke, die nur zirkulär oder ostensiv definierbar sind,

Ebene 1: Ausdrücke, deren Definitionen nur Ausdrücke der Ebene 0 , jedoch ohne Zirkularität enthalten,

Ebene 2: Ausdrücke, deren Definitionen nur Ausdrücke der Ebene 0 und der Ebene 1, jedoch ohne Zirkularität enthalten,

Ebene n: Ausdrücke, deren Definitionen nur Ausdrücke der Ebenen $0,1,2, \ldots n-1$ enthalten." (WEINREICH $1967,38)$

Das Konzept des erweiterten Lexikons der SemS, das die Konstrukteure des MST entwickeln, weist unubersehbare Parallelen zu dem Stratifikationsverfahren aus, das WEINREICH in Erwägung zieht. Einzelne Lexeme, sofern sie erst einmal in einer ihrer Bedeutungen mit Hilfe der Sems expliziert worden sind, können ihrerseits als Ausdrücke der Sems in deren erweitertes Lexikon aufgenommen werden. Auf diese Weise wird es möglich, bei den Explikationen der Bedeutungen weiterer Lexeme die gewuinschte Oberschaubarkeit und Okonomie der entsprechenden lexikographischen Definitionen herbeizuführen:

"... obwohl das Lexikon der semantischen Sprache ökonomischer als das Lexikon der naturlichen Sprache ist, ist es andererseits weit weniger flexibel und daher in mancher Hinsicht unhandlich. Insbesondere muB betont werden, daB die Explikation einer ziemlich komplexen Bedeutung der natuirlichen Sprache direkt in den Termini elementarer Bedeutungen derart umfangreich sein wiurde, daB es praktisch sehr schwierig sein durfte, mit ihr zu arbeiten. Wir sind gezwungen, neben dem .... Lexikon der Sems über irgendein erweitertes Lexikon $z u$ verfuigen, das beliebige Kombinationen aus elementaren Bedeutungen enthält, die in der zu beschreibenden natiurlichen Sprache einen monolexematischen Ausdruck (odnos Zovnoe vyrazenie) haben." (APRESJAN 1974b, 77)

APRESJAN demonstriert die Bedeutungsexplikation unter Rekurs auf das erweiterte Lexikon der Sems u.a. anhand der folgenden formalisierten lexikographischen Definitionen der Lexeme pribyvat', priešăt', prichodit', privodit' und privozit'll 
sowie anhand der wortsprachlichen lexikographischen Definitionen der Lexeme pereměsčat'sja und $\underline{i d t i}$ in einer ihrer Bedeutungen:

(1) $A$ pribyvaet $v B=$

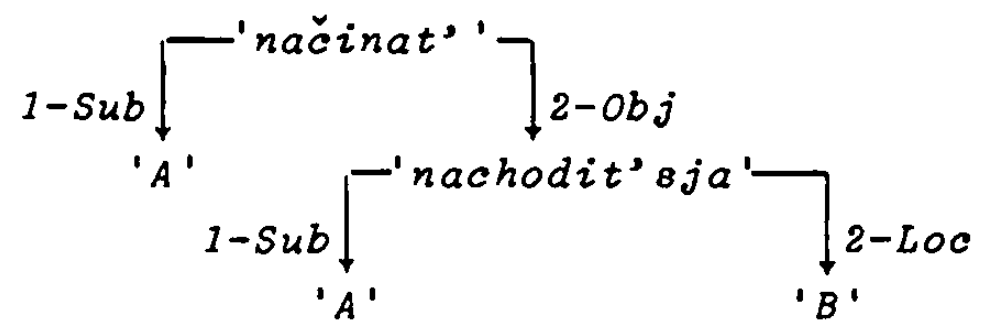

(2) $A$ prichodit $v B=$

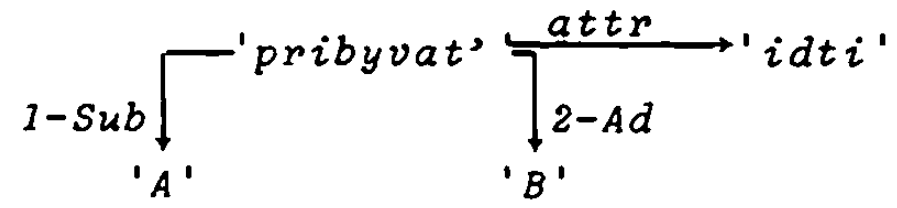

(3) A priež̃aet $v B=$

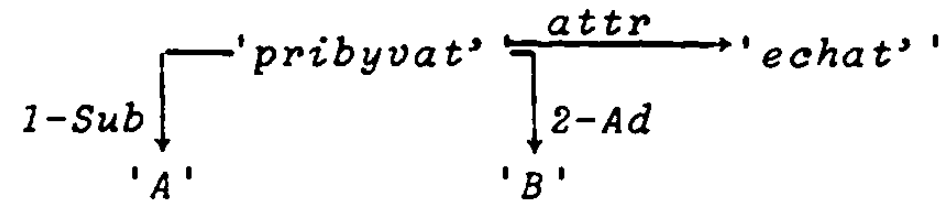

(4) A privodit $B$ v $C=$
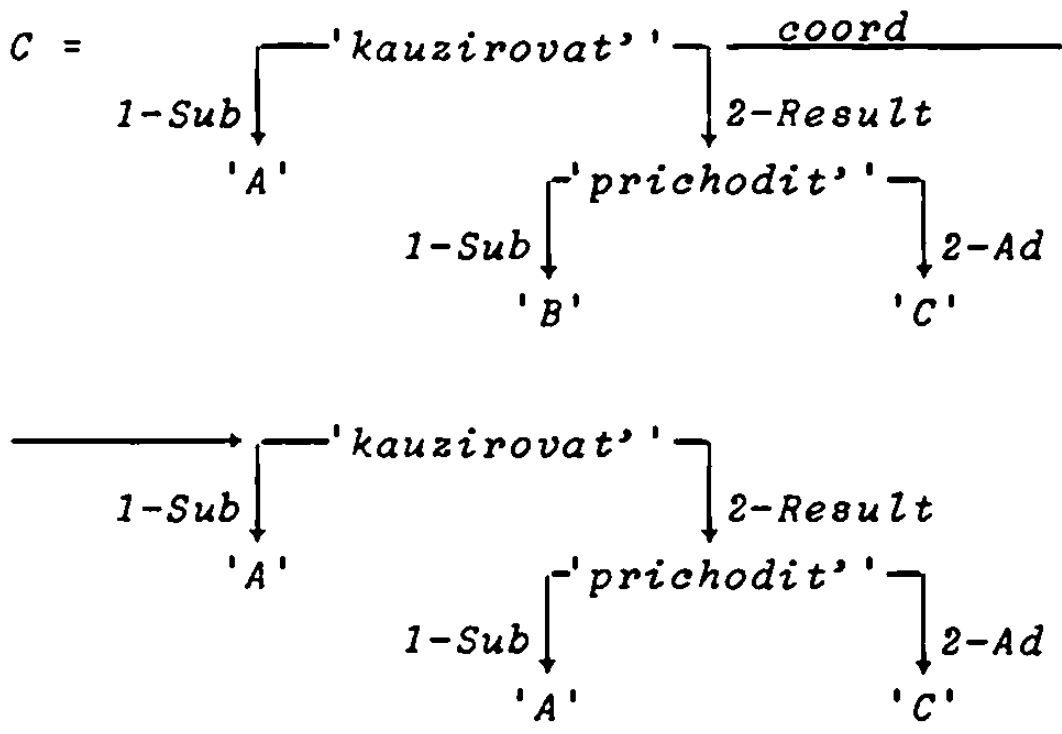

(5) A privozit B v C =

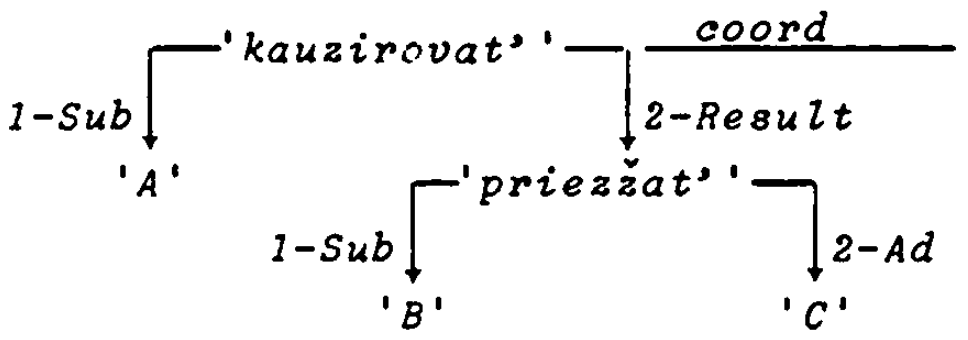




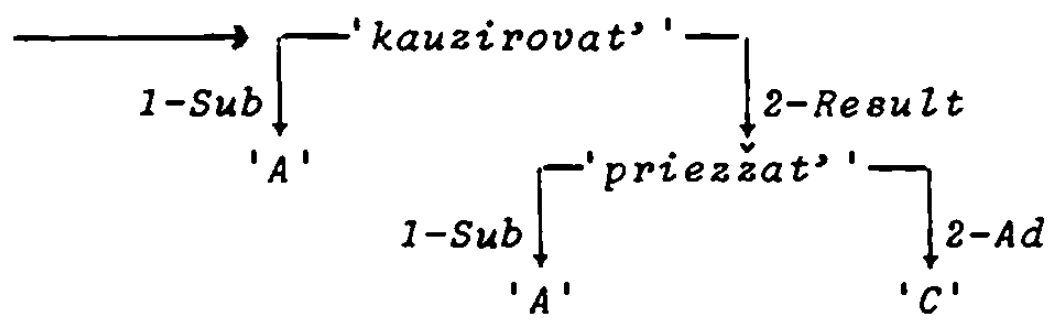

(APRESJAN 1969C, 422)

(6) A pereměsčaetsja iz $x-a v y=$ ' $V$ moment $T_{1} A$ nachoditsja $v x-e, i$

$v$ moment $T_{2} A$ nachodits $j a$

$v y-e, i$

$X$ ne sovpadaet $8 y-o m$, $i$ $T_{1}$ ne soupadaet $8 T_{2}$.'

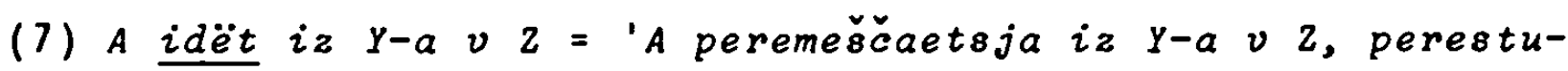
paja nogami $i$ ni $v$ kakoj moment ne utračivaja polnost'ju kontakta s poverchnost'ju, po kotoroj A peremeščatsja.'

(APRESJAN 1974b, 77, 108)

Im Gegensatz zum elementaren Lexikon der SemS, das die Konstrukteure des MST eventuell in einem zukünftigen Entwicklungsstadium als ein endliches und abgeschlossenes Inventar konzipieren, verbietet es das Konstruktionsprinzip, das dem erweiterten Lexikon der Sems zugrunde liegt, dieses in ähnlich kanonischer Weise zu betrachten. Beim erweiterten Lexikon handelt es sich vielmehr um ein offenes Wörterbuch, dessen Bestand mit der Menge der Lexeme wächst, die mit Hilfe der Sems expliziert werden.

Da die Syntax der Sems fast vollständig in ihrem Lexikon enthalten ist, führt die Erweiterung ihres Lexikons auch zu einer VergröBerung der Anzahl ihrer Dependenzrelationen. Beispielsweise werden aus der Lokal- und der Temporalvalenz, die zu den elementaren Relationen in der gegenwärtigen Entwicklungsstufe der Syntax der Sems gehören, für ihre erweiterte Syntax die komplexere Anfangspunkt- (' $A b^{\prime}$ ') und End- 
punktvalenz ('Ad') hergeleitet, so daB beispielsweise das Lexem peremeššat'sja (s.o.) als Ausdruck des erweiterten Lexikons der Sems mit den folgenden (formal und wortsprachlich notierten) inhaltlich spezifizierten Valenzen zur Explikation weiterer Lexeme eingesetzt werden kann:

$$
\begin{aligned}
& \text { 'peremeššat'sja' bzw. 'pereměsčat'sja' (A, iz } B-a, v C) \\
& 1-s u b \downarrow^{\downarrow}{ }^{\prime} \quad 2-A b \underset{B^{\prime}}{\downarrow} \downarrow^{\prime} C^{\prime}
\end{aligned}
$$

Neben der Herleitung komplexer Valenzen von Ausdrücken des erweiterten Lexikons der Sems aus solchen, die als elementare Relationen im elementaren Lexikon der SemS enthalten sind, sieht APRESJAN die Möglichkeit vor, die syntaktischen Mittel der Sems auszubauen, indem bestimmte Bezeichnungen für elementare Prädikate und elementare Gegenstände des Lexikons der Sems in inhaltich spezifizierte Dependenzrelationen umgeschrieben werden:

"Eine Erweiterung der Syntax der semantischen Sprache wird dadurch erreicht, daB einige Wörter der Klassen (1) und (2) durch syntaktische Dependenzen dargestellt werden. Das Prädikat 'ispol'zovat'sja' wird als

Med, 'pol'zovat'sja' als $\stackrel{\text { Instr }}{\longrightarrow}$, 'kauzirovat' als $\stackrel{\text { Caus }}{\longrightarrow} \mathrm{dar}$ gestellt, auBerdem werden die Gegenstandsbezeichnungen

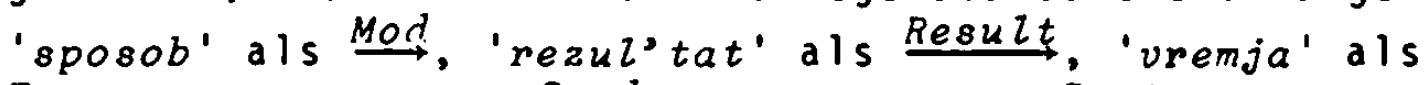
TemR, 'uslovie' als $\stackrel{\text { Cond }}{\longrightarrow}$, und 'cel" als $\stackrel{\text { Dest }}{\longrightarrow}$ dargestellt." (APRESJAN 1969c, 420)

Es muB jedoch betont werden, daß es sich bei dieser Art von Erweiterung der Syntax der Sems lediglich um eine Oberfiurung von wortsprachlich ausgedrückten Einheiten der Sems in formal notierte syntaktische Relationen handelt. Einen Beitrag, um die Oberschaubarkeit der lexikographischen Definitionen des EKW für seine potentiellen Benutzer zu erhöhen, leistet dieses Verfahren daher nicht. 
2.2.1.1.4 Zur Heuristik der Auffindung der Ausdrijcke der semantischen Sprache

Die Konstrukteure des MST verfügen über keine Auffindungsprozedur, mit deren Hilfe es möglich wäre, die Ausdrücke der Sems auf intersubjektiv nachvollziehbare Weise zu ermitteln. Auf diesen Umstand weist MEL'CUK im Zusammenhang mit der Explikation einer der Bedeutungen von tol'ko in aller Deutichkeit hin:

"Man kann die Frage stellen: auf welche Weise kann man im Wort tol'ko die genannten Bedeutungsatome $e r b l$ i c $k e n$ ? Unsere Antwort lautet: auf uberhaupt keine Weise. Bedeutungsatome sind ubrigens - genau wie die Atome der physischen Materie - nicht besonders beobachtbare, sondern postulierte GröBen." (MEL'CUK 1970, 205-206 sowie die fast identische Formulierung in MEL'CUK 1974b, 57)

Hinsichtlich einer Heuristik fïr die Auffindung der Ausdrücke der SemS bezieht APRESJAN im Vergleich zu MEL'CUK eine andere Position. Zwar kann auch er kein Auffindungsverfahren angeben, mit dessen Hilfe die "Wörter" der SemS festgelegt werden können, jedoch stellt er Oberlegungen zu verschiedenen Typen von Begriffen an, die er als geeignet betrachtet, in das Lexikon der Sems aufgenommen zu werden. In seinen Erwägungen geht APRESJAN sowohl von linguistischen als auch von nichtlinguistischen Gesichtspunkten aus:

"Als undefiniert [d.h. als elementare Bedeutungen der SemS; K.H.] betrachten wir insbesondere all die Begriffe, die in der Mathematik (vgl. 'otnosenie', 'Linija', 'ploskost' '), in der Physik ('goret' ', 'massa', 'energija'), in der Physiologie ('bolet' ") und in einigen anderen wissenschaftlichen Disziplinen definiert werden, sowie intuitiv evidente, in verschiedenen Sprachen monolexematisch ausdrückbare (odnoslouno vyrazimye) Begriffe, die nur schwer zu analysieren sind ('ponimat' 'vremja'). AuBerdem werden einige relativ einfache, jedoch nicht elementare Wörter keiner semantischen Dekomposition unterzogen, wenn ihre Explikationen zu komplex sind. Solche Wörter sind verit' ('imet' informaciju o čëm-l., vyrabotannuju sub-ektom irracional'nym putëm, bez vmesatel'stva logičeskogo myšlenija'), sčitat' ('imet' informaciju o č̈m-l., vyrabotannuju sub-ektom racional'nym putëm, $v$ rezul'tate logičskogo analiza faktov ili informacii 0 nich') u.a." (APRESJAN 1969c, 417) 
Verfahren zu verfügen, mit dessen Hilfe objektiv entschieden werden könne, ob

(a) eine semantische Dekomposition bis zur Ebene der elementaren Bedeutungen durchgeführt worden ist, und

(b) welches von zwei konkurrierenden Wörtern, die mit gleichem Recht den Status einer elementaren Bedeutung beanspruchen können, als elementareres betrachtet werden sollte:

"Erhält man Gruppen von Wörtern, für die man keine erschöpfende und redundanzfreie Definitionen mehr geben kann, die keinen tautologischen Zirkel enthalten, so ist das ein Kriterium dafür, daB die Dekomposition bis zur Ebene der elementaren Bedeutungen durchgeführt worden ist. Analysieren wir beispielsweise die Wörter zvuk und slysat'. Zvuk kann als 'to, cto vosprinimaetsja sluchom' definiert werden. Für sluch gilt jedoch 'sposobnost' vosprinimat' zvuki ili ustrojstvo dlja vosprijatija zvukov', woraus sich ein tautologischer Zirkel ergibt: in der Bedeutung des Wortes avuk ist die Bedeutung 'sluch' und in der Bedeutung des Wortes sluch die Bedeutung 'zvuk' enthalten .... In solchen Fällen wählen wir als elementar dasjenige Wort, dessen Denotat näher zur auBersprachlichen Wirklichkeit (dejstvitel'nost') steht, dessen Denotat einfacher demonstriert werden, mit einer Abbildung verbunden, gesehen, gehört usw. werden kann. offensichtlich ist es für das betrachtete Paar das Wort zvuk und nicht, wie wir früher annahmen, slysat,." (APRESJAN 1974b, 75)

Es fällt schwer, zu verstehen, worin der nach Meinung APRESJANs objektive Charakter dieser Auffindungsprozedur liegen soll. Die folgenden beiden Oberlegungen sprechen dagegen:

1) Da APRESJAN selbst die Meinung vertritt, daB elementare Bedeutungen undefiniert in das Lexikon der Sems eingefuhrt werden müssen, da sie selbst nicht expliziert werden können (APRESJAN 1969a, 12; 1969c, 418; 1974b, 76), sind die beiden oben genannten Fragestellungen im Grunde genommen widersinnig. Besteht der elementare Charakter der Ausdrücke der SemS darin, daB sie als undefinierte Einheiten angesetzt werden und somit ausschlieblich der Intuition des jeweiligen Analysators entstammen, so entzieht sich ihre Elementarität per definitionem jeglicher "objektiven" Oberpriufung. 
2) Der tautologische Zirkel, auf den APRESJAN in den beiden oben genannten lexikographischen Definitionen von zuuk und sluch stöBt, kann ohne weiteres aufgehoben werden. Die Bedeutung von zunk kann redundanzfrei und erschöpfend als 'kolebanija sredy', die Bedeutung von sluch als 'sposobnost' slyšat' ili podlě̌aščee ej ustrojstvo' definiert werden. Explizieren wir auBerdem slysat' als 'vosprinimat' uchom', so erhalten wir Definitionen von zvuk und sluch, die weder direkt noch indirekt über die Bedeutungsexplikation von slysat" zirkulär sind. Das "objektive" Kriterium, das APRESJAN in der angeblich unausweichlichen Zirkularität der Definitionen erblickt, wird somit unhaltbar.

2.2.1.1.5 Der ontologische Status der Ausdrücke der semantischen Sprache

Eng verbunden mit dem Problem der Auffindung der Ausdrücke der Sems sind auch die unterschiedichen Annahmen, die die Konstrukteure des MST zum ontologischen Status der elementaren Bedeutungen machen, welche sie in der semantischen Beschreibung verwenden. Für MEL'Cuk handelt es sich bei den Ausdruicken der Sems um linguistische Konstrukte, die angesetzt werden, um Bedeutungen von Lexemen sowie semantische Relationen zwischen Lexemen zu beschreiben (s.0.). MEL'CUKs eindeutige Position, die elementaren Bedeutungen der Sems auf keinen Fall als Erscheinungen der sprachlichen Wirklichkeit anzusehen, sondern sie ausschlieblich als GröBen der linguistischen Beschreibung aufzufassen, ergibt sich aus dem Anspruch, den er der Sprachbeschreibung durch das MST zuweist:

"In der Terminologie $N$. CHOMSKYs gesprochen, modellieren wir die 'linguistic competence' von Sprechern (ihre sprachlichen Kenntnisse) und nicht ihre 'linguistic performance', d.h. wir beschreiben nicht reale Prozesse der Verwendung, die Sprecher und Hörer von ihren Kenntnissen in sprachlichen Kommunikationsakten machen." (MEL'CUK 1974, 15) 
"Insofern als der Linguist als solcher sich mit der direkten neurophysiologischen ... Erforschung dessen, was genau im Gehirn beim Sprechen und Hören abläuft, nicht beschäftigt und - zumindest gegenwärtig - auch nicht befassen soll, nimmt die Umformungssprache [d.h. die naturliche Sprache, die als Umformer $z$ wischen Texten und Bedeutungen arbeitet; K.H.] für die Linguistik die Rolle des allgemein bekannten "schwarzen Kastens' ein." (MEL'CUK 1974b, 13)

APRESJAN vertritt im Vergleich zu MEL'CUK zum ontologischen Status der elementaren Bedeutungen eine Auffassung, aus der sich nicht ohne weiteres daruber Klarheit gewinnen $1 \ddot{B} t$, ob er sie als Größen der linguistischen oder der sprachlichen Wirklichkeit ansieht. Einerseits charakterisiert APRESJAN die SemS als eine küntliche Metasprache (vgl. S. $35 \mathrm{f.}$, andererseits finden sich in einigen seiner Arbeiten Hinweise, die die Vermutung nicht ganz unbegründet erscheinen lassen, daß er die elementaren Bedeutungen der SemS mit psychischen Entitäten identifiziert. APRESJAN geht davon aus, daB es erforderlich sei, um die Sprachbeherrschung des Menschen ( $\mathrm{gll}$. S. $3 \mathrm{ff.}$ ) zu beschreiben,

"... die Existenz einer besonderen, der direkten Beobachtung nicht zugänglichen 'semantischen Sprache' anzunehmen, in der ein Gedanke aufgeschrieben und in der Psyche des Menschen gespeichert wird." (APRESJAN 1967, 9)

Die semantische Sprache wird von APRESJAN auch als Gedankensprache (jazyk mys $l i$ ) bezeichnet:

"Die Definition des Begriffs der 'Sprachbeherrschung' setzt die Existenz einer besonderen, nicht direkt beobachtbaren 'Gedankensprache' oder semantischen Sprache voraus, in der jeder Gedanke über eine einzige, kanonische Ausdrucksform verfügt." (APRESJAN 1974b, 40)

Diese Stellungnahmen zeigen, daB APRESJAN den Terminus "semantische Sprache" zumindest in uneindeutiger Weise verwendet. In den beiden oben genannten Feststellungen bezieht er sich mit Hilfe dieses Terminus auf Gedanken (= Bedeutungen?), die in der Sprecherpsyche gespeichert sind, während er sonst unter der sems ein Beschreibungsinstrument versteht, das fur linguistische Zwecke geschaffen ist. Die mangelnde begriffiche Strenge, mit der APRESJAN den Terminus "semantische Sprache" benutzt, kann allerdings auch beabsichtigt sein 
und deshalb zu der Vermutung AnlaB geben, daB er den elementaren Bedeutungen psychische Realität zuschreibt. Eine entsprechende Haltung APRESJANs läBt sich auch aus dem Anspruch herleiten, den er mit der spezifischen Strukturierung und Darbietung des Wortschatzes im EKW verbindet. Nach APRESJAN sind die Wörterbuchartikel des EKW so angelegt,

"... daB sie die Hinführung (navedenie) eines Sprechers zum erforderlichen Ausdruck für einen Gedanken oder sogar zu dem erforderlichen Gedanken selbst gewährleisten."

(APRESJAN 1968, 42)

APRESJAN illustriert die stevernde Funktion, die das EKW fïr den Ausdruck der Gedanken seiner Benutzer angeblich haben soll, anhand des Kernbegriffs 'sozdavat' ', der den Bedeutungen der faktitiven Verben zugrunde liegt:

"Nehmen wir einmal an, ein Sprecher möchte eine Situation beschreiben, in der ein Subjekt im Laufe seiner Tätigkeit irgendein Objekt hervorbringt. Die allgemeine Bezeichnung fur diese Situation ist wohl das Verb sozdavat'. Je nach dem, welches objekt hervorgebracht wird lein Buch - kniga, ein Musikstiuck - muzykaz'noe proizvedenie, eine Skulptur skul'ptura u.a.), werden die Artbegriffe (vidovye ponjatija) pisat', socinjat', vajat' u.a. unterschieden. Aus ihnen kann der Sprecher diejenigen auswählen, die seinen Gedanken genau ausdrücken." (APRESJAN 1968, 43)

Die Identifizierung elementarer Bedeutungen mit psychischen Entitäten ist in Semantiktheorien, die von der generativen Linguistik beeinfluBt worden sind, weit verbreitet. Im ersten Kapitel von APRESJAN 1974b, das den Titel "Quellen der Semantik" (Istoki semantiki) trägt, bespricht der Autor ausfihrlich u.a. die Semantikkonzeptionen von FILLMORE und WIERZBICKA, deren (quasi) semantische Metasprachen im Mittelpunkt seines Interesses stehen (APRESJAN 1974b, 24-36). APRESJANs gründiche Rezeption der Arbeiten von FILLMORE und WIERZBICKA ist wohl auch dafür verantwortlich zu machen, daB er dazu neigt, den elementaren Bedeutungen der SemS einen ontologischen Status im oben genannten Sinne zuzusprechen. FILLMORE vertritt in der Arbeit "The Case for Case" (FILLMORE 1968), in der er den ersten Ansatz zu seiner Kasusgrammatik entwickelt, die Auffassung, daB kognitive Vorgänge in der Sprecherpsyche u.a. vermittels der von ihm spezifizier- 
ten Tiefenkasus ablaufen:

"Die Kasusbegriffe umfassen eine Menge universaler, vermutlich angeborener Konzepte, die bestimmte Typen von Urteilen identifizieren, die Menschen über Vorgänge fällen können, welche in ihrer Umgebung ablaufen - Urteile daruber, wer etwas getan hat, wem eine Sache zugestoßen ist und was sich verändert hat." (FILLMORE 1968, 24)

WIERZBICKA bindet ihre Konzeption der metasprachlichen semantischen Analyse an sprach- und erkenntnisphilosophische Strömungen des 17. Jahrhunderts. Für sie liegt das Ziel der Semantik

"... in der Suche nach universalen, nicht arbiträren 'Elementen des menschlichen Denkens'." (WIERZBICKA 1972, 3)

WIERZBICKA entwickelt eine semantische Metasprache, die aus sogenannten semantischen Primitiven besteht. Die Autorin geht davon aus, daß diese Metasprache psychische Realität besitze, denn sie behauptet, daß in der semantischen Sprache die menschliche Kognition vor sich gehe. WIERZBICKA bezeichnet ihre Metasprache auch als lingua mentalis (WIERZBICKA 1972, 25). Mit ihr will sie eine Forderung einlösen, die schon LEIBNIZ erhoben hat, nämlich ein System von Konzepten zu konstruieren, mit deren Hilfe der Mensch begriffliche Operationen vornimmt. LEIBNIZ bezeichnet ein solches System als Alphabetum Cogitationum humanarum:

"Das Alphabet der menschlichen Gedanken (Alphabetum Cogitationum humanarum) ist eine Zusammenstellung derjenigen Gedanken, die von selbst verstanden werden und durch deren Kombination unsere ubrigen Ideen entstehen." (zitiert nach WIERZBICKA 1972 , 6)

Zweifellos stellt die in der linguistischen Semantik weit verbreitete Auffassung, elementare Bedeutungen als real in der menschlichen Psyche existierende Größen mit gnoseologischer Funktion zu betrachten, eine interessante Behauptung dar, denn sie betrifft die häufig diskutierte, jedoch bis heute immer noch ungelöste Frage nach dem Verhältnis von Sprache, Denken und Erkenntnis. Worin ihr Beitrag sowohl fuir die semantische Beschreibung als auch die Theoriebildung in der Linguistik bestehen soll, ist uns al- 
lerdings nicht $\mathrm{klar}$. Derartige Behauptungen zum ontologischen Status der elementaren Bedeutungen entziehen sich - zumindest im gegenwärtigen Stand linguistischer Forschurig jeglicher Oberprüfung, die, wenn überhaupt, auch eher von der Sprachpsychologie zu erwarten sein durfte. Dieser Umstand sollte, sofern man aus ihm überhaupt konsequenzen für die linguistische forschung ziehen will, jedoch nicht dazu verleiten, vorläufig von Bemuhungen in der Linguistik, die Inhaltsseite sprachlicher Zeichen, d.h. ihre grammatischen und ihre lexikalischen Bedeutungen zu beschreiben, in der zuversichtlichen Erwartung abzulassen, daß eines Tages der empirische Nachweis der psychischen Realität elementarer Bedeutungen erbracht werde.

Der hohe Anspruch an die linguistische Semantik, eine sogenannte starke oder externe Semantik zu sein, der sich zwangsläufig ergeben muß, wenn elementare Bedeutungen mit psychischen Entitäten identifiziert werden, erscheint uns unrealistisch. Wir schließen uns vielmehr der Auffassung MEL'CUKs an, der es für angemessen hält, innerhalb der Linguistik die Erforschung der Bedeutung sprachlicher Zeichen ausschlieBlich mit dem Anspruch einer sogenannten schwachen oder internen Semantik zu betreiben (MEL'CUK 1974b, 23-25, 76). Die Aufgaben einer schwachen Semantik bestehen nach der Ansicht von MEL'CUK sowohl in der strukturellen Beschreibung der Einheiten eines gegebenen Wortschatzes als auch in der Beschreibung ihrer Bedeutungen (vgl. S. $66 \mathrm{ff}$.). Die Frage, wie Sprecher mit $\mathrm{Hilfe}$ der Bedeutungen sprachlicher Zeichen Referenz auf die auBersprachliche Wirklichkeit erstellen, d.h. Sie wahrnehmen oder erkennen, gehört für MEL'CUK dagegen nicht zum kreis der Probleme, mit denen sich die schwache, d.h. die linguistische Semantik befassen soll:

"... außerdem gibt es die sogenannte $s t a r k$ Semantik - das Problem der Denotation (nominacija), d.h. der Aufstellung von Regeln, nach denen sprachliche Zeichen außersprachlichen Gegenständen zugeordnet werden, das Problem der Ermittlung von Wahrheitswerten sprachlicher AuBerungen usw. Für sich genommen sind diese Dinge erforderlich, wichtig und interessant, allerdings gehen sie weit über die eigentlichen Grenzen der Linguistik hinaus, da sie die Klassifikation und Beschreibung einer sehr großen Menge außersprachlicher Ob- 
jekte sowie die Beschäftigung mit Bedeutungen, die ohne Bezug zu ihrer sprachlichen Manifestation untersucht werden, voraussetzen." (MEL'CUK 1974b, 76)

Die Auffassung MEL'CUKs zu Eigenschaften sprachlicher Zeichen, deren Beschreibung in den Zuständigkeitsbereich der linguistischen Semantik fallen soll, bietet eine hinreichende Begründung dafür, die Andeutungen zur psychischen Realität elementarer Bedeutungen, die in bestimmten AuBerungen APRESJANs zu finden sind (s.o.), völlig aus der linguistischen Semantik zu verbannen. Den folgereichen Implikationen, die die Aufrechterhaltung einer derartigen Position nach sich ziehen wïrde, könnte die Linguistik mit den Beschreibungsverfahren, über die sie zur Zeit verfügt, sicher nicht gerecht werden.

Indem wir uns MEL'CUKs Auffassung zum Untersuchungsbereich der linguistischen Semantik zueigen machen, wird für uns auch die in der generativen Linguistik oft diskutierte Frage gegenstandslos, ob ein Grammatikmodell semantisch oder syntaktisch zentriert werden soll (vgl. S. 5), sofern sie ausschlieBlich durch oberlegungen zur Gerichtetheit (directionality) des Sprechakts aufgeworfen wird (CHAFE 1971, 6-9). Da es sich beim MST analog zur generativen Transformationsgrammatik CHOMSKYs um ein Kompetenzmodell handelt (vgl. S. 3), ist es unzulässig, allein aufgrund von Erwägungen, die die Performanz betreffen, eine feste und gerichtete Reihenfolge der Beschreibungsebenen zu postulieren (CHOMSKY 1971, 187 ff.). Aus diesem Grund halten wir die Feststellung, die IMMLER zum semantisch zentrierten Grammatikmodell der generativen Semantik macht und die - gestiutz durch APRESJANS Position im Hinblick auf den ontologischen Status der SemS (s.o.) - erst recht an die Adresse des MST gerichtet werden könnte, für nicht stichhaltig:

"... die generative Semantik behauptet .... daB alle sprachlich vermittelte Wahrnehmung der Wirklichkeit einer einzigen Grundstruktur folgt: der eines Prädikats und eines oder mehrerer Argumente, über die durch dieses Prädikat etwas ausgesagt wird." (IMMLER 1974, 212-213)

Als unrealistisch und für die linguistische Forschung nicht 
sachdienlich betrachten wir daher auch sein Postulat, das er nicht nur speziell für die generative Semantik, sondern wohl auch für die linguistische Semantik im allgemeinen erhebt:

"Aufgabe einer wirklichen generativen Semantik wäre es aber, zu zeigen, wie bestimmte BewuBtseinsinhalte sprachlichen Ausdruck finden können, wie mitzuteilenden Inhalten eine semantische Repräsentation zugeordnet wird und wie diese dann in syntaktisch-phonetischen Strukturen realisiert werden können. Ein wichtiger Bestandteil einer solchen Theorie ist die Behandlung der Frage, wie Aspekte der Wirklichkeit durch sprachliche Zeichen abgebildet werden können, und damit eine Erklärung, wie wir uns mit Hilfe der Sprache auf die Wirklichkeit beziehen, von ihr sprechen können - also eine Theorie des Zusammenhangs von Referenz und Bedeutung." (IMMLER 1974, 216)

2.2.1.1.6 Der künstliche Charakter der semantischen Sprache

Die Konstrukteure des MST betrachten die Definitionssprache des EKW als eine "künstliche" semantische Sprache ("iskusstvennyj" semantičeskij jazyk). Unter dem künstlichen Charakter der SemS des EKW verstehen sie eine Reihe struktureller Eigenschaften, welche sie von der naturlichen Sprache, die in gängigen Definitionswörterbüchern eingesetzt wird, um Wortbedeutungen zu explizieren, ihrer Ansicht nach erheblich unterscheiden (s.u.).

In unterschiedlicher Weise bewerten die Konstrukteure des MST die Vereinbarkeit des von ihnen als künstlich bezeichneten Charakters der Sems mit der Tatsache, daB in ihr bis auf wenige Ausnahmen ausschlieBlich russische Wörter verwendet und sogar in ihrer wortsprachlichen (= lexikographischen) Notationsvariante zumindest die Regeln der russischen Grammatik durchweg beachtet werden.

MEL'CUK unterstreicht ausdrücklich, daB es eines der Konstruktionsprinzipien der Sems sei, Ausdrücke der Objektsprache, d.h. des Russischen, in ihren Bestand aufzunehmen. Den meta- oder kunstsprachlichen Status der russischen Ausdrücke, die als "Wörter" der sems verwendet werden, erblickt er darin, daB u.a. ihre Bedeutungen im Vergleich zum lobjekt- 
sprachlichen) Russischen stark reglementiert werden sollen. MEL'CUK beabsichtigt, das Referenzpotential bestimmter russicher Lexeme, die als metasprachliche Einheiten in die künstliche Definitionssprache eingehen, zu reduzieren, indem er ihre Bedeutungsumfänge derart einschränkt, daß jeder Ausdruck in der sems nur noch in genau einer Bedeutung für die semantische Beschreibung eingesetzt wird. M.a.W., aus der Menge derjenigen russischen Lexeme, die in der Sems zur Verwendung gelangen, sollen bestimmte Ausdrücke monosemiert werden:

"Die Sprache Basic [d.h. die semantische Sprache des MST in seiner ersten Entwicklungsstufe, die der SemS des EKW entspricht, vgl. S.14; K.H.J ist keine universale Hilfssprache, die auf der Grundlage von Logik oder logischer Semantik konstruiert worden ist; sie grijndet sich vielmehr auf das Prinzip der maximalen Nähe zu den natürlichen Sprachen ... Die lexikalischen Mittel von Basic haben 'nationalen' Charakter. Das Basic Russian [d.h. die semantische Sprache, mit deren Hilfe die russische Lexik beschrieben wird; K.H.] ist eigentlich ein Präparat des Russischen in dem Sinne, daB die Elemente die dafür ausgewählt werden, ihre 'russischen Bedeutungen', also ihre 'russischen Begriffsumfänge' im großen und ganzen behalten, obwohl bei der Konstruktion von Basic bestimmte Spezifizierungen der Bedeutung der Wörter vorgenommen werden können (im Basic Russian sind beispielsweise 'zakon,' - 'juristisches Gesetz' und 'zakong' - 'Naturgesetz' verschiedene Elemente) ..." (ZOLKOVSKIJ/MEL'CUK 1967, 181)

"Nachdem man, wo es erforderlich ist, die Bedeutung von Semen präzisiert hat, kann man sie durch die entsprechenden russischen Wörter bezeichnen." (MEL'CUK 1974b, 73)

Die Monosemierung eines russischen Lexems für seinen Gebrauch in der Sems demonstriert MEL'CUK am Beispiels des verbs borot'sja:

"... 'borot'sja' hat ... eine ziemlich komplexe Explikation. Wir verwenden nur eine der Bedeutungen des russischen Verbs $\underline{\text { borot's } j a}=$ 'pytat'sja ustranit' $v$ kačestve prepjatstvija'."

Während MEL'CUKs Aussagen zum künstlichen Charakter der SemS keinen $A n l a B$ bieten, einen Widerspruch zwischen der Tatsache, daB die Sems in Russisch abgefaBt ist, und ihrer Einstufung als einer Kunstsprache zu erblicken, drängen gewisse Ausfiuhrungen APRESJANs zur Kiunstlichkeit der SemS die- 
sen Widerspruch geradezu auf.

APRESJAN scheint das von MEL'CUK dargestellte Konstruktionsprinzip, als Seme in die Sems russische Lexeme aufzunehmen, nicht zu akzeptieren. Im Gegensatz zu MEL'CUK, der auf die Abhängigkeit der Sems von der Objektsprache Russisch ausdrücklich hinweist, erwecken APRESJANS Aussagen den Eindruck, daß es sich bei der Objektsprache Russisch und der künstlichen Sems um zwei völlig verschiedene Dinge handele. Den Umstand, daß in der Sems fast ausschließlich russische Ausdrucksmittel verwendet werden, scheint APRESJAN als eine vorläufig unvermeidbare, äuBerlich bedingte Begleiterscheinung zu betrachten, die allein durch das geringe AusmaB begründet ist, in dem die sems bisher ausgearbeitet worden ist. Gerade in diesem Sinne äußert er die Vermutung, daß es möglich sein werde, sobald die Sems das Ausgangsstadium eines Provisoriums uberwunden habe, ihr Inventar an "nationalen" Ausdrucksmitteln durch ein anderes, nicht russisches zu ersetzen:

"... im russisch-semantischen Lexikon [d.h. in der Sems des EKW; K.H.] werden die zu definierenden Einheiten, d.h. die Ausdrücke der naturlichen Sprache, und die definierenden Einheiten, d.h. die Wörter der semantischen Sprache (= die elementaren Bedeutungen) deutlich unterschieden. Dieser prinzipielle Unterschied zwischen der Sprache, die beschrieben werden soll, ... und der Sprache, mit deren Hilfe beschrieben wird, .... bleibt auch in dem Fall aufrechterhalten, wenn aus irgendwelchen äuBeren Gründen die Bedeutungsexplikation faktisch in den Wörtern derselben Sprache abgefaBt wird. Er bleibt deswegen bestehen, weil alle Explikationen eine ganz bestimmte logische Struktur aufweisen: sie sind auf eine kleine Menge einfachster, $u n d e f i$ $n i$ e $r$ e $r$ Begriffe zurückführbar..." (APRESJAN 1966, 260)

"... die verschiedenen elementaren Bedeutungen werden durch Wörter der russischen Sprache bezeichnet. In Zukunft wird es vielleicht erforderlich sein, darauf zu verzichten und symbolische Bezeichnungen für die elementaren Bedeutungen auf der Grundlage des Lateinischen wie im Basic von A.K. ZOLKOVSKIJ und I.A. MEL'CUK einzuführen." (APRESJAN 1969c, 416)

"... die elementaren Bedeutungen werden hauptsächlich durch russische Wörter bezeichnet (vgl. jedoch 'kauzirovat' '). In der Zukunft, wenn sich in der Linguistik die Umrisse eines Lexikons der semantischen Sprache deutlicher abzeichnen, 
wird es vielleicht erforderlich sein, auf solche Bezeichnungen $z u$ verzichten und $z u$ einer internationalen semantischen Transkription' ijberzugehen ...; heute wäre das noch zu früh, jedoch darf die Verwendung fiktiver Wörter, d.h. von Bezeichnungen der naturlichen Sprache, denen andere (nur partiell identische) Bedeutungen zugewiesen worden sind, vor uns die Tatsache nicht verbergen, daß zwischen den beiden entsprechenden Objekten ein prinzipieller Unterschied besteht." (APRESJAN 1974b, 74)

APRESJANS Auffassung, daB es sich bei der Sems als einer künstlichen Metasprache und der Objektsprache Russisch um zwei völlig verschiedene Gegenstände handele, die nur zufällig äußerliche Gemeinsamkeiten in bestimmten Ausdrücken aufweisen, welche in ihnen verwendet werden, prinzipiell jedoch unabhängig voneinander seien, betrachten wir als völlig unhaltbar. Es ist unverständlich, wieso APRESJAN "irgendwelche äußerlichen Gründe" dafür verantwortlich macht, daB in der SemS des EKW Bedeutungsexplikationen fast ausschließlich unter Rückgriff auf Ausdrucksmittel des Russischen vorgenommen werden. Gerade aus diesem Umstand bezieht die SemS doch die wichtige Eigenschaft, sowohl für den Linguisten als auch den Wörterbuchbenutzer verstehbar zu sein, ohne daß jede einzelne Einheit, die sie benutzt, spezifiziert wird. Die lexikographischen Definitionen, die in der Sems abgefaßt sind, können nur aufgrund ihrer weitgehenden Abhängigkeit von der Objektsprache Russisch verstanden, hinsichtlich ihrer Güte beurteilt und gegebenenfalls korrigiert werden.

Der kunstliche Charakter der Sems ist auch, so wie ihn APRESJAN versteht, mit der Zweckbestimmung des EKW unvereinbar. Die Konstrukteure des MST, von denen sich zumal noch APRESJAN besonders intensiv mit der lexikographischen Variante der SemS des EKW beschäftigt hat, wollen ein Wörterbuch erstellen, das praktische lexikographische Bedurfnisse seiner potentiellen Adressaten u.a. im Bereich der Wortdefinitionen befriedigen soll. Wie aber können die Bedeutungsexplikationen dieses Wörterbuchs seinen potentiellen Benutzern zugänglich sein, wenn in ihnen eine Definitionssprache (wie beispielsweise "symbolische lateinische Bezeichnungen") verwendet wird, die sie nicht verstehen? 
Für die Behauptung REUTHERs, daB die SemS des EKW von dessen potentiellen Adressaten vorab zu erlernen sei, haben wir in den Arbeiten der Konstrukteure des MST keinen Hinweis gefunden:

"Die Formulierung der Erklärungen [d.h. der Bedeutungsexplikationen; K.H.] in einer speziell erarbeiteten (und vom Benutzer zu erlernenden!) semantischen Sprache stellt eine wesentliche Neuerung in der Lexikographie dar." (REUTHER $1978,26)^{12}$

Für ein Lexikon, das seine Urheber u.a. mit dem Anspruch entwickeln, mit gängigen Definitionswörterbüchern des Russischen zu konkurrieren, ergäbe sich im übrigen aus der Tatsache, daB dessen Definitionssprache zunächst zu erlernen ist, ein nahezu unüberwindlicher Nachteil. Eine weitere Schwierigkeit bestünde darin, Wege zu finden, die Sems des EKW in angemessener Weise lehren zu können.

Der Versuch APRESJANs, der SemS des EKW den Anschein eines küstlichen Gebildes zu verleihen, das zwar als Metasprache zur semantischen Beschreibung des Russischen effektiv eingesetzt werden könne, jedoch im Grunde genommen von diesem völlig unabhängig sei, so daB es in Zukunft auch durch symbolische Notationen abgelöst werden könne, ist symptomatisch für die Gleichgïltigkeit, die die Konstrukteure des MST dem potentiellen Adressatenkreis ihres Wörterbuchs entgegenbringen. Die Aufstellung von lexikologischen Prinzipien und ihre lexikographische Anwendung bei der Beschreibung eines Wortschatzes setzt die Entscheidung über den Kreis von Sprechern voraus, an die sich das Wörterbuch wenden soll. Den Konstrukteuren des MST ist vorzuwerfen, daB sie diesen Zusammenhang fast völlig auBer acht lassen, obwohl sie ganz dezidiert den Anspruch erheben, ein neuartiges Wörterbuch verfassen zu wollen, das u.a. gängigen Definitionswörterbüchern des Russischen weit iberlegen sein soll. 13 APRESJANS Ausführungen zum künstlichen Charakter der SemS des EKW, in der die Frage ihrer Verstehbarkeit für potentielle wörterbuchbenutzer überhaupt nicht angesprochen und die obereinstimmung ihrer Ausdrucksmittel mit denen der objektsprache Russisch als Zufallsprodukt dargestellt wird, 
lassen diesen Mangel deutlich zutage treten.

Wir wollen uns nicht unbedingt dagegen wenden, die Sems des EKW als eine "künstliche" Metasprache zu bezeichnen. Allerdings sollte dann diesem Terminus nur der Begriff von "Künstlichkeit" zugrundeliegen, den MEL'CUK mit inm verbindet (s.o.). Die "künstliche" semantische Metasprache ist demzufolge nichts anderes als ein Ausschnitt des Russischen, der in mehrerer Hinsicht stark reglementiert worden ist. Diese Reglementiertheit äuBert sich u.a. in dem Versuch, die russischen Wörter, welche in die Sems aufgenommen werden, zu monosemieren. Die Trennung zwischen der Objektsprache Russisch und der "künstlichen" SemS des EKW kann dann nur noch in der Form aufrechterhalten werden, daB es sich bei letzterer um ein Fragment der objektsprache handelt, das nach entsprechender Reglementierung als Metasprache eingesetzt wird, um die objektsprache semantisch zu beschreiben. Ganz in diesem Sinne äuBert sich auch WIERZBICKA, die von der prinzipiellen Nichthintergehbarkeit der natiurlichen Sprache ausgeht und daher Kunstsprachen als Beschreibungsinstrumentarien fü die natürliche Sprache ablehnt. WIERZBICKA erblickt den einzigen Ausweg darin, für die semantische Forschung die Eigenschaft der naturlichen Sprache auszunutzen, selbsterklärend zu sein:

"Meiner Meinung nach besteht der fruchtbarste Ansatz in dem Versuch, die Bedeutungsrepräsentationen gleichzeitig zu ihren Erkiärungen zu machen. Diese Art der semantischen Repräsentation ... ist nur möglich, wenn die Notation grundsätzlich selbsterklärend ist. Die semantische Metasprache ist nur dann wirklich 'erklärend', wenn sie so klar und unmittelbar verstehbar ist, daB sie ihrerseits keine 'Erklärung' erfordert... Kunstsprachen sind nicht selbsterklärend. Sie entstehen auf dem Hintergrund der naturlichen Sprache und sind letztlich nur iber diese verstehbar. Sie haben keinen direkten Kontakt zur Intuition, während die natïrliche Sprache mit dieser verankert ist." (WIERZBICKA 1972, 1-2)

WIERZBICKA schlägt deshalb vor, einen Teil der naturlichen Sprache auszugrenzen, inn durch Reduktion auf wenige Ausdruicke, die sogenannten semantischen Primitive, zu reglementieren und als Metasprache für die semantische Beschreibung der natiurlichen Sprache zu verwenden.

Der selbsterklärende Charakter der natülichen Sprache ver- 
anlaBt auch PADUCEVA dazu, eine semantische Metasprache zu fordern, die ausschlieBlich unter Rekurs auf die natiurliche Sprache zu konstruieren ist:

"Wir glauben, daß von den verschiedensten semantischen Sprachen diejenige vorzuziehen ist, die ein Fragment der zu beschreibenden natülichen Sprache sein kann. Wir wollen diese Präferenz das $P R$ I N Z I P DER NATOR $L$ I C H K E I T (princip estestvennosti) nennen. M.a.W., es ist wijnschenswert, soweit möglich, die natürliche Sprache als ihre eigene Metasprache zu verwenden ... In der Tat kann die Unschärfe, die der naturlichen Sprache eigen ist, wenn es gewiunscht wird, überwunden werden. Es ist im ibrigen offensichtlich, daB diese Auswahl die Aufgabe erleichtern wird. Eine solche Sprache erfordert keine zusätzliche Interpretation: sie ist durch sich selbst verstehbar, während fur eine beliebige künstliche Sprache dennoch letztlich in derselben natürlichen Sprache eine Interpretation beschrieben werden muB." (PADUCEVA 1974, 16)

Eine ähnliche Position bezieht auch WEINREICH, der ebenfalls die semantische Metasprache als einen Ausschnitt aus der natülichen Sprache betrachtet, der in seinen Ausdrucksmitteln stark reduziert worden ist:

"Im Idealfall könnten wir uns eine 'absolute' Metasprache wünschen, die von der Objektsprache oder von jeglicher natürlichen Sprache völlig unabhängig ist. Da dieser Idealfall jedoch in der Semantik (im Gegensatz zur Phonetik) illusorisch ist, sollte man Wege suchen, um die Metasprache eher weniger umfangreich als genauso umfangreich oder gar noch umfangreicher als die objektsprache zu machen." (WEINREICH 1967, 37)

2.2.1.1.7 Die Struktur der Ausdrücke der semantischen Sprache

Kann man einerseits einen Gegensatz konstatieren, in den sich APRESJAN mit seinen Ausfuihrungen zum künstichen Charakter der SemS zu MEL'CUK begibt, so wird andererseits aus den Postulaten, die er an die Ausdrücke der SemS des EKW stellt, deutlich, daß er implizit die gleiche Position wie MEL'CUK vertritt und die Sems als ein Fragment des Russischen auffaBt, das strengen strukturelien Restriktionen unterworfen ist.

Die Konstrukteure des MST erheben zwei Postulate, denen die 
Ausdrücke der SemS des EKW genügen sollen. Die beiden Postulate, die die Sems im Vergleich zur Objektsprache Russisch stark reglementieren, betrachten sie als Idealforderungen, denen die SemS zur Zeit nur annäherungsweise genigen könne. Die erste Bedingung, die die Einheiten der SemS erfiullen missen, besteht darin, daB zwischen ihren Inhalten und Ausdrucken eine ein-eindeutige Beziehung zu bestehen habe:

"Das Lexikon der semantischen Sprache muB (im Idealfall, der bisher von niemanden erreicht worden ist) den folgenden Bedingungen genügen: jedes seiner Wörter muß genau eine, möglichst eine elementare Bedeutung ausdruicken, und jede elementare Bedeutung muB durch genau ein Wort der semantischen Sprache ausgedruickt werden, ganz unabhängig davon, in welcher Explikation sie vorkommt (die elementaren Bedeutungen und ihre Benennungen müssen im Verhältnis der gegenseitig-eindeutigen Entsprechung stehen). Wird beispielsweise ein Teil der Bedeutung des Adjektivs korotkij mit $\mathrm{Hilfe}$ des Wortes 'nebol'soj' expliziert, so muB dieser in den Explikationen der Adjektive nizkij uzkij, tonkij, lëgkij und anderer ähnlicher Lexeme durch dasselbe Wort reprasentiert werden und nicht durch die Wörter 'malyj', 'neznacitel'nyi' u.ä., wie es in Definitionswörterbiuchern gängigen Typs üblich ist. Anderersejts darf man nicht, sofern $u z k i j$ als 'nebol'soj $v$ poperečnike' und sirokij als 'bol'sojv poperečnike' expliziert werden, tonkij als 'nebol'soj $v$ poperečnike' und tolstyj als 'bol'soj v poperečnike' explizieren. Der Ausdruck voperečnike' ernält sonst faktisch zwe $i$ Lesarten - ' $v$ sirinu' für das erste Antonympar und ' $v$ tolšcinu' für das zweite. M.a.W., im Lexikon der semantischen Sprache darf es weder Synonymie noch Homonymie der Bezeichnungen für die elementaren Bedeutungen geben." (APRESJAN 1974b, 70́; vgl. auch die partiell fast identischen Formulierungen in APRESJAN 1969b, 77 und 1969c, 415)

Die zweite Bedingung, der die Ausdrücke der SemS genuigen müssen, besteht darin, daB ihr Bestand im Vergleich zur Anzahl der Wortschatzeinheiten, die durch sie semantisch beschrieben werden können, wesentlich geringer zu sein hat. Gleichzeitig sollen deren Bedeutungen, vorausgesetzt, sie gehören zur sogenannten abstrakten Lexik (vgl. S. 108 ff.), mit Hilfe des Inventars der Sems erschöpfend beschrieben werden:

"Okonomie in dieser Beschreibung (d.h. der Beschreibung mittels der SemS; K.H.l wird dadurch erzielt, daB die Anzahl der Einheiten der Metasprache ... um einige Stellen niedriger ist als die Anzahl der Einheiten der zu beschreibenden Sprache, die eine erschöpfende (eindeutige) Charakterisierung erfahren soll." (APRESJAN 1967, 10-11) 
"Die Anzahl der elementaren Bedeutungen muB auBerdem gering sein (Okonomiepostulat), jedoch hinreichend, damit alle lexikalischen Bedeutungen im Rahmen des ausgegrenzten Gegenstandsbereiches [d.h. der sogenannten abstrakten Lexik; K.H.] in erschöpfender Weise beschrieben werden können (Vollständigkeitspostulat)." (APRESJAN 1974b, 71; 1969c, $415)$

Um zu erreichen, daB zwischen den Ausdrucksmitteln der Sems und ihren Inhalten weder Synonymie noch Homonymie besteht, bedienen sich die Konstrukteure des MST zweier Verfahren, zu denen sie allerdings kaum theoretische Oberlegungen anstellen:

1) Sie versuchen, indem sie die Bedeutungen bestimmter russischer wörter, die in die Sems übernommen werden, präzisieren, die Beziehung zwischen deren Inhalt und deren Ausdruck so stark einzuschränken, daB zwischen beiden die postulierte ein-eindeutige Entsprechung angesetzt werden kann. Um die gewüschte Zuordnung von Inhalt und Ausdruck der Einheiten der Sems herbeizufihren, schlagen die Konstrukteure des MST zwei verschiedene Wege ein. Sie spezifizieren die Bedeutung bestimmter russischer wörter, indem sie versuchen,

a) deren Referenzpotential so weit zu reduzieren, daB die betreffenden wörter monosemiert (oder disambiguiert) werden;

b) ihnen eine Bedeutung zuzuweisen, die mit der Bedeutung, in der sie im Russischen verwendet werden, nur partiell ubereinstimmt.

Wir wollen dieses Verfahren, mit dessen Hilfe die Konstrukteure des MST allerdings nur eine ganz geringe Anzahl von Ausdrücken der SemS gewinnen, anhand einiger der wenigen Beispiele, die sich in ihren Arbeiten finden, demonstrieren.

In einer seiner Veröffentlichungen zum EKW führt APRESJAN eine Liste von Bezeichnungen für elementare Prädikate und elementare Gegenstände an, die Bestandteil des Lexikons der Sems ist. Bestimmte Ausdrucke dieser Liste hat APRESJAN durch Zusätze in Klammern kommentiert, mit denen er versucht, die betreffenden wörter zu monosemie- 
ren (vgl. auch S.34 MEL'CUKs Monosemierung von 'borot'sja'). Beispielsweise werden 'dezat' ' durch (čto-libo), 'ispol'zovat' durch (upotrebljat' kak sredstvo), 'pol'zovat'sja' durch (primenjat' $v$ kačestve instrumenta), 'realizovat' durch (osuščestvijat'), 'sčitat" durch (polagat'), 'izmerenie' durch (prostranstvennoe) und 'mir' durch (vněsnij) spezifiziert (APRESJAN 1969c, $416)^{14}$.

Es muB bezweifelt werden, ob APRESJAN durch diese Zusätze bei allen oben genannten elementaren Bedeutungen eine hinreichende Monosemierung herbeigefüht hat. Zumindest die Spezifizierung (čto-libo) bei 'dezat' dürte kaum geeignet sein, das Referenzpotential des Wortes einzuschränken. Beabsichtigt APRESJAN, durch den Zusatz 'ćto-libo' das Lexem so zu monosemieren, daB sein Inhalt auf den Begriff reduziert wird, der sämtlichen faktitiven Verben zugrundeliegt und als 'sozdavat' ' angegeben werden kann? Oder will er 'dezat' čto-Zibo' wie beispielsweise in den Wortverbindungen delat' šag, vyvod, ošibku im Sinne von 'dejstvovat' tak, čtoby ...' als ein Sem verwenden, das in der semantischen Beschreibung zur Bezeichnung von solchen Handlungen eingesetzt wird, die durch eine menschliche Kausalinstanz vollzogen werden? Weiterhin ist zu bedenken, $d a B$ bei der Auswahl anderer russischer Lexeme als Einheiten der Sems einige der oben genannten Zusätze überflüssig werden. Dies wäre dann der Fall, wenn beispielsweise nicht die homonymen russischen Verben scitat' und realizirovat, sondern die Verben polagat' und osusccestuljat' in die sems übernommen würden.

Als sogenanntes fiktives Wort, d.h. als ein Lexem, dessen Bedeutung er in einer Weise festlegt, daB sie mit der Bedeutung, in der das Wort im Russischen gebraucht wird, nur teilidentisch ist, betrachtet APRESJAN das Sem 'norma', das er zur Bedeutungsexplikation von parametrischen Adjektiven verwendet. Anhand der Definition des Adjektivs dalëkij $=$ 'dal'še normy' demonstriert APRESJAN, welche Bedeutung er dem fiktiven Wort 'norma' zuweist: 
"Norma ist ein undefiniertes Wort, jedoch muB seine Bedeutung erläutert werden. Wenn $X$ sich zu einem Ort B begibt und einen Vorübergehenden $Y$ fragt, ob es weit bis $B$ sei, so können bei real derselben Entfernung von $B$ zu dem Ort, an dem $X$ und $Y$ sich getroffen haben, die Antworten von $Y$ sehr verschieden ausfallen: daleko, ne sliskom, sredne, nedaleko, blizko, rukoj podat'. Was ist also die Norm der Entfernung (norma rasstojanija)? Um diese Frage zu lösen, muß man verstehen, wodurch sich die Streuung der Antworten erklärt. Offensichtlich hängen die Antworten von $Y$ l) von der Bewegungsart von $X$ .... 2) von der Geschwindigkeit seiner Bewegung .... 3) vom Zustand des Wegs und einigen anderen Faktoren ab. Aus diesem Beispiel folgt, daB die Norm (norma) eine Sachlage bezeichnet, die sich für die Mehrheit der Sprecher als die wahrscheinlichste in der gegebenen konkreten Situation darstellen muB (oder darstellt)." (APRESJAN 1974b, 74)

Unter die fiktiven Wörter subsumieren die Konstrukteure des MST auch solche Seme, die sie in Anlehnung an russische Wortbildungsparadigmen schaffen. Dieser Typ fiktiver Wörter umfaBt solche Ausdrücke, die nur Abkürzungen für Bedeutungen sind, die im Russischen periphrastisch ausgedrückt werden müssen. Hierzu zählen beispielsweise die Seme 'vachtit' = 'nesti vachtu' und 'prestupat' ' = 'soveršat' prestuplenie' (ZOLKOVSKIJ/MEL'CUK 1967, 181) und 'pustota' = 'pustoe prostranstvo $v$ tele, ograničennoe telom a) so vsech storon...., ili b) so vsech storon, krome odnoj..., $i l_{i}$ c) so vsech storon, krome duuch protivopoložnych ...' (APRESJAN 1974b, 74-75). Da die Bedeutungen dieser Ausdrücke komplex sind, gehören sie dem erweiterten Lexikon der Sems an.

AuBerdem enthält das Lexikon der Sems noch das fiktive Wort lateinischen Ursprungs 'kauzirovat' ', das die Konstrukteure des MST jedoch nicht inhaltlich spezifizieren. ${ }^{15}$ Wir werten diesen Umstand als einen weiteren $B e-$ leg für die Gleichgiultigkeit, die sie den potentiellen Benutzern des EKW entgegenbringen. Eine Kommentierung dieses Sems - vorausgesetzt, man will an inm festhalten und es nicht beispielsweise durch 'pričinjat" ersetzen (vgl. S. $47 \mathrm{f.}$ ) - ist unbedingt erforderlich, da es in sehr vielen lexikographischen Definitionen verwendet wird, die für ihre potentiellen Adressaten ansonsten 
unverständlich bleiben dürten.

Indem die Konstrukteure des MST versuchen, den Inhalt bestimmter russischer wörter zu reglementieren, um diese dann als metasprachliche Einheiten für die semantische Beschreibung nutzbar machen zu können, bedienen sie sich eines Verfahrens, das in der linguistischen Semantik nicht unbekannt ist. Im Zusammenhang mit der Merkmalanalyse weist beispielsweise auch KATZ darauf hin, daB die Wörter, die zur Bezeichnung der semantischen Merkmale eingesetzt werden, nicht in der gleichen Bedeutung verwendet werden, die ihnen in der entsprechenden objektsprache zukommt:

"Die semantischen Markierungen repräsentieren die Begriffselemente, in die eine Lesart einen Sinn aufgliedert... Der Hinweis ist wichtig, daB die semantischen Markierungen, wenngleich sie in der Orthographie der natürlichen Sprache erscheinen, nicht mit den Wörtern oder Ausdrücken der Sprache identifiziert werden können, die bei diesen andeutenden Benennungen benutzt wurde." (KATZ 1971,142 )

Anhand des deutschen Wortes Gewässer zeigt SCHWARZE, wie mit Hilfe eines sogenannten Bedeutungspostulats - im Sinne der Autoren des FUNK-KOLLEGS SPRACHE (FUNK-KOLLEG SPRACHE 1973, Bd. II, 93-94) - der Inhalt des Lexems in einer Weise festgelegt werden kann, daß es als semantisches Merkmal in eine semantische Metasprache aufgenommen werden kann:

"So ist es Z.B. intuitiv leicht einzusehen, daB Meer, See, Teich, Tümpel und Pfütze gemeinsam Hyponyme eines Oberbegriffs 'Gewässer sein könnten. Im Deutschen gibt es aber ein solches Wort nicht. (Gewässer hat zwar Meer, Teich, See, vielleicht auch Tümpel, aber sicher nicht pfitze als Hyponym!) Ich kann also die Beziehung zwischen Meer und Pfiutze mit Hilfe der Sinnrelation nicht darstelTen. Mit der Methode der Merkmalsanalyse hingegen ist dies kein Problem: ich kann ein Merkmal 'Gewässer' annehmen, das sich inhaltilich mit dem deutschen Wort Gewässer nicht zu decken braucht." (SCHWARZE 1975, 99)

2) Die Konstrukteure des MST übernehmen Wörter des Russischen in die Sems, ohne die Zuordnung zwischen ihrem Ausdruck und ihrem Inhalt besonders zu vereinbaren oder fest- 
zulegen. Auf diese Weise gewinnen sie die weitaus größte Zahl von Einheiten der SemS. Sie scheinen dabei einerseits in Anlehnung an WIERZBICKA und PADUCEVA auf die selbsterklärende Kraft der natürlichen Sprache zu vertrauen. Andererseits scheinen sie davon auszugehen, daB es ihnen weitgehend gelingt, bei der semantischen Beschreibung ein russisches Wort in der Sems nur so einzusetzen, daB es immer als Bezeichnung für dieselbe Bedeutung verwendet wird. Gleichzeitig gehen sie jedoch das Risiko ein, daB die Inhalte, die verschiedene Analysatoren oder Wörterbuchbenutzer mit den "Wörtern" der SemS assoziieren, nicht denen entsprechen, von denen ihre Konstrukteure ausgehen, so daB leicht Diskussionen um die Gïte einer lexikographischen Definition entstehen können. Als ein Beispiel dafür, daB inhaltlich unspezifizierte Einheiten der SemS, die die Konstrukteure des MST in lexikographischen Definitionen verwenden, zu unterschiedlichen Auffassungen über deren Adäquatheit führen können, wollen wir die Kritik nennen, die KOTELOVA an den Bedeutungsexplikationen übt, die das EKW für die Lexeme metod und staraja deva gibt.

Diese beiden Stichwörter werden wie folgt definiert:

(1) $\underline{\text { metod }}=$ 'Sposob delat' čto-libo, osnovannyj na dostatočno složnych intellektual'nych postroenijach'

(2) staraja deva $=$ 'Zenščina, davno dostigšaja polovoj zrelosti, no ne sumeviaja naladit. normal'nuju polovuju żizn' $i l i$ votupit' $v$ brak' (zitiert nach KOTELOVA $1975,28,29$ )

Wir halten KOTELOVAS Vorwurf, daB beide Explikationen zum Teil polyseme Wörter enthalten, für berechtigt. Dies gilt für die Seme 'delat', 'složnyj' und 'umet' ', denen man in beiden Definitionen verschiedene Lesarten zuordnen kann. Sollen in (1) 'delat' als 'dejstvovat' ', 'postupat' oder 'sozdavat' čto-libo' und 'složnyj' als 'trudnyj' oder 'sostojaščij iz neskol'kich častej' verstanden werden? Ist in (2) der Semkomplex 'ne sumevsaja' als 'kotoroj ne udalos'. 
oder 'kotoraja ne byza sposobnaja' zu interpretieren? KOTELOVAS SchluBfolgerung, daB u.a. aufgrund der Polysemie, die fur die Einheiten der SemS des EKW angeblich durchweg charakteristisch sei, diese für lexikographische Definitionen prinzipiell untauglich sei, können wir dagegen nicht akzeptieren. Die Mehrdeutigkeit bestimmter Ausdrücke, die von den Konstrukteuren des MST ohne jegliche inhaltiche Reglementierung in die Sems übernommen werden, stellt zwejfellos einen Mangel dar, jedoch ist er ohne weiteres durch eine hinreichende Monosemierung der betreffenden Einheiten zu beseitigen.

2.2.1.1.8 Probleme der Synonymie und Homonymie in der semantischen Sprache

Wir haben bereits darauf hingewiesen, daB die konstrukteure des MST die Reglementierung, der die Zuordnung von Inhalt und Ausdruck sämtlicher "Wörter" der SemS unterliegen soll, als eine Idealforderung bezeichnen, der die Definitionssprache des EKW im gegenwärtigen Entwicklungsstand nur näherungsweise entspricht (vgl. S. 40). In der Tat läBt sich nachweisen, daB die SemS des EKW sowohl in ihrem Lexikon als auch in ihrer Syntax synonyme und homonyme Ausdrucksmittel enthält. 16

Innerhalb derselben Veröffentlichung zur semantischen Beschreibung im EKW, die demzufolge auch auf eine Sems zurückgreifen mübte, welche zumindest im Rahmen dieser Publikation homogen zu sein hat, verwenden die konstrukteure des MST beispielsweise für den Begriff des Besitzens von Dispositionen und für den Begriff des Verursachens - im folgenden als Kausation bezeichnet - synonyme bzw. partiell synonyme Ausdrücke. Obwohl er im Lexikon der Sems nur das Sem 'imet" anfürt, um den Begriff des Besitzens zu bezeichnen, den er im ibrigen hinsichtlich der verschiedenen Typen von Besitzguitern wie beispielsweise Gegenständen, Eigenschaften und Dispositionen nicht differenziert, ersetzt APRESJAN 'imet' ' in bestimmten lexikographischen Definitio- 
nen durch die Seme 'sposobnyj', 'svojstvennyj' und 'obladajuščij (sposobnost'ju)'; vgl. die Bedeutungsexplikationen (3) - (5):

(3) X spravedlivyj $=$ 'X sposobnyj pravil'no ocenit', kak sleduet dejstvovat', čtoby nikakim ijudjam ne bylo kauzirovano nezaslužennoe zlo.'

(4) $X$ ostorožen $=$ ' $X-u$ svojstvenno želanie izbegat' situacij, kotorye mogut byt' opasnymi $i z i$ neprijatnymi dija $x-a, i$ poetomu $x$ staraetsja dejstvovat, s učëtom vsech tech faktorov, kotorye, po ego mneniju, mogut kauzirovat' takuju situaciju."

(5) $X \underline{\text { lovkij }}=$ ' $X$ - obladajuščij sposobnost'ju bystro $i$ bez truda koordinirovat' dviženija svoego tela pri vypolnenii fizičeskich dejstvij.' (APRESJAN 1974b, 107) 17

Für den Begriff der Kausation sieht APRESJAN im Lexikon der Sems die Bedeutungskomponente 'kauzirovat' ' vor. In bestimmten lexikographischen Definitionen ersetzt er sie jedoch durch die Seme 'pričinjat" und 'vyzyvat' '; vgl. die Bedeutungsexplikationen (6) - (8):

(6) A kopiruet $X=$ 'A sozdaët $X$ ', pytajas' kauzirovat' maksimal'noe schodstvo me żdu $x$-om $i X^{\prime}$-om.'

(7) $A$ b ët $Y-a \quad X-o m=$ 'A udarjaet $Y-a \quad X$-om mnogo raz podrjad, starajas' pričinit, $y-u$ fizičeskuju bol'.'

(8) Ljubov' $X-a k y-u=$ 'Cuvstvo, ispytyvaemoe X-om po otnoseniju $k \dot{y}-u$, kotoryj prijaten $x-u$ $i$ vyzyvaet $u x-a$ zelanie byt'v kontakte $s$-om $i l_{i}$ kauzirovat' y-u dobro.' (APRESJAN 1974b, 107. $108,109)$

In der lexikographischen Definition von udarjat' verwendet APRESJAN anstelle des Sems 'kauzirovat' den Semkomplex 'privodit' $v$ kontakt'; vgl. die Bedeutungsexplikation (9): 
(9) X udarjaet po $y$ - 2 -om $=$ 'X rezko i kratkovremenno privodit kompaktnyj predmet $y$ $v$ kontakt s predmetom 2.' (APRESJAN 1974b, 108)

Im Gegensatz zu den Bedeutungsexplikationen (7) und (8) liegt in der Bedeutungsexplikation (9) allerdings keine totale Synonymie zwischen dem Semkomplex 'privodit' $v$ kontakt' und 'kauzirovat' vor. Bei dem Ausdruck ' $X$ privodit $Y v$ kontakt ...' handelt es sich vielmehr um eine abkürzende Schreibweise für einen Teil der Bedeutung, die die Konstrukteure des MST dem kausativen Verb udarjat' zuweisen. Diese Teilexplikation des Stichworts beinhaltet 'kauzirovat' neben anderen Bedeutungskomponenten: ' $X$ privodit $Y$ vontakt ...' = 'X kauziruet $Y$ prichodit' $v$ kontakt...'.'

Auch MEL'CUK substituiert in bestimmten lexikographischen Definitionen 'kauzirovat' durch 'pričinjat' '; vgl. die Bedeutungsexplikationen (10) - (11):

(10) X soobščaet $Y-u$, čto $P=' X$ éksplicitno kauziruet to, čto Y znaet, čto $P$.'

(11) $A$ nakazyvaet $B$ za $C D$-om = 'A pričinjaet $B-u$, soveršivsemu prostupok $C, z$ zo $D$ s cel'ju ispravit' $B$ ili podobnych emu lic.' (MEL'CUK $1974 b, 63,134$ )

Um die erforderliche Verstehbarkeit der lexikographischen Definitionen für die potentiellen Benutzer des EKW herbeizufiuhren, wäre es im übrigen angebracht, auf 'kauzirovat' ' in der Sems ganz zu verzichten und beispielsweise 'pričinjat'. zu verwenden, wie es auch die Konstrukteure des MST in (7) und (11) tun (vgl. S. 187). Auf diese Weise wären die Bedeutungsexplikationen des Wörterbuchs auch potentiellen Adressaten zugänglich, die nicht über Lateinkenntnisse verfügen. Synonymie im Lexikon der SemS des EKW entsteht auch dadurch, daB die Konstrukteure des MST in verschiedenen Arbeiten unterschiedliche Seme fur denselben Begriff einsetzen. So wird in APRESJAN 1969 C der Begriff der Bewegung durch 'dvigat'sja', in APRESJAN $1974 \mathrm{~b}$ dagegen durch 'peremeščat'sja' 
ausgedrückt; vgl. die Bedeutungsexplikationen (12) - (13): (12) A udaljaetsja ot $B-a=$ 'Rasstojanie meždu A-om i B-om uveličivaetsja i A dvižetsja.' (APRESJAN 1969C, 423)

(13) A udaljaetsja ot $B-a=$ 'A peremě̌čaetsja, i rasstojanie ot $A-a$ do B-a uveličivaetsja.' (APRESJAN 1974b, 108)

Das Vorhandensein der oben genannten synonymen Ausdrücke im Lexikon der SemS hat für die Güte der semantischen Beschreibungen des Russischen im EKW keine tiefgreifenden Konsequenzen, da sich die Synonymie dieser Ausdriucke durch eine hinreichende Standardisierung der Bezeichnungen für die oben genannten Begriffe beseitigen läBt. Zu schwerwiegenden Folgen nicht nur für die Adäquatheit der lexikographischen Definitionen des EKW, von denen die Konstrukteure des MST U.a. Vollständigkeit fordern ( $v g l$. S. $67 \mathrm{ff.}$ ), sondern auch für die Intention des Gesamtmodells, Paraphrasebeziehungen zwischen Texten zu beschreiben, führt dagegen die Existenz des homonymen Sems 'kauzirovat' ' im Lexikon der SemS. Diese Bedeutungskomponente, die in zahlreichen lexikographischen Definitionen des EKW verwendet wird, betrachten die Konstrukteure des MST als eine elementare Bedeutung. M.a.W., sie gehen von einem undefinierten Begriff der Kausation aus, den sie trotz seiner Komplexität in der semantischen Beschreibung ausschlieblich durch das Sem 'kauzirovat' 'bzw. durch seine oben genannten Kquivalente bezeichnet. Wir wollen zwei Aspekte, die zur Komplexität des Begriffs der Kausation beitragen, näher untersuchen. Hierbei handelt es sich einmal um die Rolle, welche die räumliche Distanz, d.h. der direkte bzw. der indirekte kontakt zwischen den Aktanten spielt, die in eine von ihnen verursachten Handlung involviert sind, und zum anderen um die Frage, ob der Verursacher einer Handlung diese vorsätzlich (intentional) vollzieht. Wir betrachten den Begriff der Kausation unter diesen beiden Fragestellungen, weil die Konstrukteure des MST im Zusammenhang mit dem Sem 'kauzirovat' ' den Aspekt der räumlichen Distanz zwischen den entsprechenden Aktanten 
implizit berücksichtigen, dem Aspekt der Intentionalität bei der Verwendung von 'kauzirovat' ' dagegen ausdrücklich jede Relevanz für die semantische Beschreibung absprechen.

Im Zusammenhang mit der Bedeutungsexplikation, die von der nordamerikanischen generativen Semantik für das Verb to $k i l l=$ 'to cause to die' gegeben worden ist, bespricht WIERZBICKA - in Anlehnung an Oberlegungen von KATZ - das Problem der direkten bzw. der indirekten Kausation. WIERZBICKA zeigt, daB to kill im Gegensatz zu to cause to die nur verwendet werden kann, wenn der Handlung eine direkte Kausation zugrunde liegt. M.a.W., zwischen dem Verursacher und dem Mitspieler, der von der Handlung im Sinne von FILLMORE 1968, 4 und von WELTE 1974, 421 affiziert wird, darf keine räumliche Distanz bestehen, die einen unmittelbaren Kontakt zwischen beiden unmöglich macht:

"Hat jemand den Tod einer Person indirekt verursacht (to cause), wird man nicht sagen, er habe inn getötet (to kill). Katz' $k$ leine Wildwest-Parabel über den Sheriff, dessen sechsschuissiger Colt von einem ortsansässigen Waffenschmied fehlerhaft repariert worden ist, so daB die Waffe im kritischen Augenblick versagt und er niedergeschossen wird, illustriert dies auf ziemlich lustige Art. Katz gelangt zu der SchluBfolgerung, daB der Waffenschmied den Tod des Sheriffs eindeutig verursacht habe, jedoch habe der Waffenschmied ihn genauso eindeutig nicht getötet." (WIERZBICKA 1975, 492)

Erklärt man das objektsprachliche Lexem to cause zu einem metasprachlichen Ausdruck und reduziert man sein Referenzpotential nicht auf eine direkte oder auf eine indirekte Kausation, so ist es unmöglich, fur bestimmte Wörter mit Hilfe dieses Sems Explikationen zu geben, die die Bedeutungen der Explikanda vollständig erfassen. Wird eine entsprechende Disambiguierung von 'to cause' nicht vorgenommen, ergibt sich Homonymie in der semantischen Beschreibungssprache. Die Bedeutungen solcher Wörter, die - wie beispielsweise die Gruppe der faktitiven Verben - fast ausschlieBlich (s.u.) im oben genannten Sinne direkt kausierte Handlungen bezeichnen, könnten daher weder eindeutig noch vollständig erfaßt werden.

Bei der semantischen Beschreibung des russischen Wortschatzes trägt MEL'CUK dem Problem der Direktheit bzw. Indirekt- 
heit der Kausation einer Handlung dadurch Rechnung, daB er fiur die Explikation des Verbs poit', das nur eine direkt kausierte Handlung bezeichnen kann, das Sem 'kauzirovat' ' durch die Bedeutungskomponente 'neposredstvenno' modifiziert; vgl. die (von uns wortsprachlich notierte) lexikographische Definition (14):

(14) $X$ poit $y-a \quad z-o m=' X$ neposredstvenno kauziruet $Y-a$ pit' 2.' (MEL'CUK 1974b, 70)

Während MEL'CUK den Begriff der Kausation bezüglich der Rolle, die die räumliche Distanz zwischen den an der entsprechenden Handlung beteiligten Aktanten spielt, in der semantischen Beschreibung differenziert, verwendet APRESJAN das Sem 'kauzirovat' ' in dieser Hinsicht undifferenziert. Die lexikographischen Definitionen (15) - (18), die APRESJAN für die Verben vešat', vybivat', prizyvat' und ubě̌dat' in einer ihrer Bedeutungen gibt, zeigen dies ganz deutlich. 0 bwohl vešat' und vybivat' im Gegensatz zu prizyvat' und ubeždat' eine nur direkt kausierte Handlung bezeichnen können, benutzt er für die Explikation aller vier Stichwörter das unmodifizierte 'kauzirovat' ':

(15) A vešaet $B$ na $C=$ 'A kauziruet $B$ viset' na $C-e$. '

(16) A vybivaet $B$ iz C-a X-om = 'A kauziruet $B$ perestavat' nachodit'sja $v$ C-e, udarjaja c $x$-om.'

(17) A prizyvaet $X-a k y-u=$ 'Obrä̌čajas' $k y-u$, A pytaetsja kauzirovat' $X-a$ sdelat' $Y$.

(18) A ubě̃daet $X-a v Y-e=$ 'Privodja argumenty, A pytaetsja kauzirovat' $X-a$ sčitat' $Y$.' (APRESJAN 1974b, 75, 205, 83)

Die Homonymie von 'kauzirovat' in den Bedeutungsexplikationen (15) - (18) wäre ohne weiteres dadurch zu beseitigen, daß man - analog zu MEL'CUKs Vorgehen - in das Lexikon der SemS des EKW eine elementare Bedeutung 'neposredstvenno' aufnimmt, welche immer dann attributiv mit 'kauzirovat" " verknüpft wird, wenn Lexeme expliziert werden sollen, die man im Hinblick auf die direkte Kausation der Handlung, die 
sie bezeichnen, als markiert betrachten kann.

Neben der direkten kausation besteht eine weitere semantische Eigenschaft, die fur einen Teil der Kausative paradigmatisch ist, in der intentionalen Einbezogenheit eines bestimmten Mitspielers in die Handlung. Im Zusammenhang mit der semantischen Beschreibung faktitiver Verben, die sie - in Anlehnung an LYONS (LYONS 1972, 450) - existentielle Kausative nennt, weist WIERZBICKA darauf hin, daB der Aktant, dem in der jeweils bezeichneten Situation die Agensrolle zukommt, die Handlung stets intentional vollzieht. 18 Aus der Gruppe der Kausativa kann daher die klasse der faktitiven Verben ausgegrenzt werden, die man hinsichtlich der intentionalen Einbezogenheit des Agens in die Handlung, die sie bezeichnen, als markiert ansehen kann. Zweifel an der Wohlgeformtheit der Sätze (19) und (20) im Gegensatz zu der von (21) veranlassen WIERZBICKA zu der Forderung, in der semantischen Beschreibung der faktitiven Verben oder existentiellen Kausativa die Bedeutungskomponente 'Intentionalität des Agens' zu berucksichtigen:

"Sind die folgenden Sätze nicht ein wenig seltsam:

(19) ? Bill, unintentionally, made an omelette.

(20) ? Bill, unintentionally, built a house.

Der Satz

(21) Bill, unintentionally, killed the cat.

ist dagegen uberhaupt nicht anomal. Dieser Umstand legt es nahe, daB eine Komponente, welche die Intention des Agens anzeigt, in die semantische Repräsentation der Wörter to build, to cook (in der Bedeutung von to make) und ähnlicher aufgenommen werden sollte." (WIERZBICRA 1975, 506)

WIERZBICKAS Urteil über die Wohlgeformheit von (19) und (20) ist selbstverständlich nur dann begründet, wenn in den Sätzen das Adverb "unintentionally" als Modifikation zu "made" bzw. "built" aufgefaBt wird. Die zweite mögliche Interpretation von (19) und (20), in der der Skopus des Adverbs "unintentionally" sich auf die Ausdrücke "an omelette" bzw. "a house" erstreckt, führt zu keinem VerstoB gegen die Wohlgeformtheit der Sätze; vgl. (19') und $\left(20^{\prime}\right)$ :

(19') 8 ill, unintentionally, made an omelette (, although he 
wanted to do fried eggs).

(20') (Playing with his bricks) 8 ill, unintentionally, built a house (, although we wanted to build a garage).

Aus der Ambiguität der Sätze erklärt sich auch die Tatsache, daB WIERZBICKA (19) und (20) lediglich als relativ nichtwohlgeformt einstuft. Ihr Wohlgeformtheitsurteil grïndet sich zweifellos auf die implizite Annahme, daB die Lesart von (19) und (20), in der durch das Adverb "unintentionally" nicht die Verbhandlungen, sondern die Resultate dieser Handlungen näher bestimmt werden, zwar denkbar ist, jedoch erheblich von den typischerweise durch die Verben to make und to build bezeichneten außersprachlichen situationen abweicht.

APRESJAN diskutiert das Problem der intentionalen bzw. der nichtintentionalen Kausation (namerennost'/nenamerennost') unter der allgemeinen Fragestellung der Polysemie. Die Sätze (22) - (23) lassen nach APRESJAN bezüglich der intentionalen Einbezogenheit des Agens keine eindeutige Interpretation zu:

(22) Rebënok dyšal.

(23) Gusar zagremel śporami. (APRESJAN 1974b, 176)

Werden die Sätze (22) und (23) mit den kausativen Verben dysat' und gremet' dagegen in die beiden folgenden größeren Textzusammenhänge eingebettet, so läBt sich eine eindeutige Lesart herbeiführen. Nach APRESJAN bezeichnet dysat' in (22') eine vom Agens intentional kausierte, gremet' in (23') eine nichtintentional kausierte Handlung:

(22') Rebënok, kak zavedennyj, otkryval rot, vysovyval jazyk, často dys̆al, i vrać sčital, čto osmotr udalsja na slavu.

(23') Lichoj gusar, naošcup' prodvigajas' po koridoru $k$ zavetnomu nomeru molodoj vdovy, spotknulsja o stupen'ku, upal $i$ zagremel šporami. (APRESJAN 1974b, 177)

APRESJAN weigert sich, die oben genannten Verben als polyseme Lexeme anzusehen. Für ihn ist die zweifache Interpretation, die die Sätze (22) und (23) zulassen, nicht das Ergeb- 
nis lexikalischer Polysemie. Für die doppelte Lesart der Sätze macht er vielmehr sogenannte auBersprachliche (enzyklopädische) Faktoren verantwortlich. Auf das Wissen, das Sprecher iber bestimmte auBersprachliche Faktoren besitzen, führt er auch die eindeutigen Interpretationen zurück, die

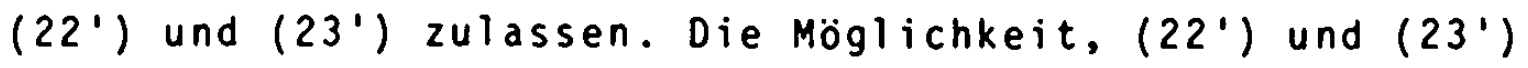
eindeutig zu verstehen, ergibt sich nach APRESJAN aus der Kenntnis der dynamischen Beziehungen, die Arzt und Patient bzw. Mann und Frau in bestimmten Situationen mit weitgehend standardisierten Handlungsabläufen eingehen können:

"Der Satz 'Rebënok ... často dyšaz' ist hier nur im 'intentionalen' Sinn zu verstehen. Diese Interpretation wird jedoch nicht durch die lexikalischen Bedeutungen der Wörter erzwungen, sondern durch bestimmte enzyklopädische Informationen über die Situation der ärztlichen Untersuchung. In gleicher Weise zwingt uns gerade das Wissen um die auBersprachliche Situation 'Husar - junge Witwe' und nicht irgendeine lexikalische Information dazu, den Satz 'Gusar... $i$ zagremel sporami' im 'nichtintentionalen' Sinn zu verstehen." (APRESJAN 1974b, 177) 19

APRESJAN gelangt zu der SchluBfolgerung, daB die Mehrdeutigkeit, die Kausativa in bestimmten Verwendungen hinsichtich der intentionalen Einbezogenheit des Agens in die Handlung aufweisen können, nicht als eine Polysemie der langue (jazykovaja mnogoznačnost'), sondern als eine auBersprachlich bedingte Polysemie der parole (rečevaja mnogoznačnost') aufzufassen sei. Indem APRESJAN das Problem der Intentionalitä, das der Begriff der Kausation aufwirft, als eine Erscheinung der parole einstuft, stellt sich für ihn auch nicht die Notwendigkeit, in der semantischen Beschreibung der Kausativa die Bedeutungskomponente 'kauzirovat' ' gegebenenfalls entsprechend zu differenzieren. Bezüglich der intentionalen Einbezogenheit des Agens in die Handlung sind Kausativa für APRESJAN daher monoseme lexikalische Einheiten. Aus dieser Auffassung folgt, daB Kausativa im Hinblick auf die hier in Rede stehende semantische Eigenschaft nicht ambig, sordern vage sind, d.h. ihre Unschärfe oder Unbestimmtheit ist nicht auf der Abstraktionsebene der langue, sondern der parole zu beschreiben. 
Das Argument, mit dem die Konstrukteure des MST das Problen der Intentionalität der Kausativa aus der semantischen Beschreibung im EKW ausklammern, muB auf dem Hintergrund der system- oder kompetenzlinguistischen Orientierung des MST gesehen werden (vgl.S. 3). In einem linguistischen Modell, das die abstrakte Fähigkeit von Sprechern beschreiben soll, mit Hilfe der Bedeutungen sprachlicher Zeichen u.a. Referenzen herstellen zu können, brauchen selbstverständlich nicht konkrete Verwendungssituationen berücksichtigt zu werden, in denen Sprecher von dieser Fähigkeit Gebrauch machen. Eine weitere Oberlegung, die für die Haltung der Konstrukteure des MST spricht, besteht darin, daB es sehr schwierig sein dürte, typische Verwendungsweisen für Kausativa in der Art, wie APRESJAN sie anführt (s.o.), herauszufinden und zusammenzustellen. Das sogenannte enzyklopädische Wissen und situative Faktoren, aufgrund derer Sprecher in bestimmten Fällen Kausativa auf die eine oder die andere Weise verstehen, entzieht sich weitgehend jeglicher systematik /vgl. in diesem Zusammenhang allerdings den Versuch in ZOLKOVSKIJ/ MEL'CUK 1969, 11-12, die Paraphrasierbarkeit von Sätzen aufgrund enzyklopädischen Wissens durch ein sogenanntes Axiom der auBersprachlichen Wirklichkeit zu beschreiben). Obwohl die Konstrukteure des MST das Problem der Intentionalität der Handlung bei der semantischen Beschreibung der Kausativa als eine Erscheinung der parole betrachten, finden sich im EKW Bedeutungsexplikationen von einigen wenigen kausativen verben, in denen die Bedeutungskomponente 'Intentionalität des Agens' zwar nicht durch einen bestimmten Ausdruck des Explikationstexts repräsentiert wird, jedoch in anderen Ausdrücken enthalten ist (SCHMIOT 1981). Um solche Einheiten handelt es sich bei den Semen 'plan', 'cel". 'starat'sja' und 'pytat'sja' in den lexikographischen Definitionen von obučat', preduprě̌dat', bit' und prizyvat'; vgl. die Bedeutungsexplikationen (24) - (27):

(24) X obuçaet $Y-a z-u=$ 'Po opredelénnomu planu $X$ kauziruet $y$-a znat' $i l i$ umet' 2 .' (APRESJAN 1974b, 85) 
(25) A preduprě̌daet. $B-a \circ C-e=$ 'A, polagaja, čto $C$

$$
\begin{aligned}
& \text { kasaetsja } B-a \text { i čto } B-u \\
& \text { sleduet reagirovat' na } C \text {, } \\
& \text { soobšcaet } B-u \text { o } C-e s \\
& \text { cel'ju, čtoby znanie o } c-e \\
& \text { zastavilo } B-a \text { reagirovat'. ' } \\
& \text { (MEL'CUK 1974b, 179) }
\end{aligned}
$$

(26) $X \underline{b^{\prime} \ddot{e} t} Y-a$ 2-om $=$ ' $X$ udarjaet $Y$-a 2-om mnogo raz podrjad, starajas' pričinit' y-u fizičeskuju bol'.' (APRESJAN 1974b, 108)

(27) A prizyvaet $X-a k y-u=$ 'obraščajas' $k$ X-u, A pytaetsja kauzirovat' $X-a$ sdelat' $Y . '$ (APRESJAN 1974b, 83)

Das Sem 'éksplicitno', das die Konstrukteure des MST zu verwenden scheinen, um sprechaktbezeichnende Verben wie soob̌̌čat', oběsčat' u.ä. von performativ verwendbaren verben wie govorit, pisat' u.ä. zu trennen, kann wahrscheinlich in gleicher Weise interpretiert werden; vgl. die Bedeutungsexplikation (28) von soobšcat':

(28) X soobšcaet $Y-u$, čto $P=' X$ éksplicitno kauziruet to, čto $Y$ znaet, čto P.' (MEL'CUK $1974 b, 63)$

Diese Beispiele diurfen allerdings nicht dariber hinwegtäuschen, daB die Konstrukteure des MST die Frage der Intentionalität der Handlung bei der semantischen Analyse von Kausativa weitgehend ignorieren.

Ihr Vorgehen zieht jedoch in bestimmten Fällen für die Gite der Bedeutungsexplikationen des EKW schwerwiegende Konsequenzen nach sich. Die Forderung, die die Konstrukteure des MST an die lexikographischen Definitionen des EKW richten, die Bedeutung des Explikandum vollständig zu erfassen (vgl. S. 67 ff.), wird in all den fällen nicht eingelöst, in denen die Intentionalität der Handlung nur als eine paradigmatische Eigenschaft der lexikalischen Bedeutung eines Kausativs beschrieben werden kann. Wir glauben, daß sich zwei Gruppen von kausativen Verben angeben lassen, deren lexikalische Bedeutungen nur in der geforderten Vollständigkeit beschrieben wer- 
den können, wenn in ihren Explikationen die intentionale Einbezogenheit des Agens in die Handlung repräsentiert wird. Hierbei handelt es sich einmal um die Klasse der faktitiven Verben. Die Sinnanomalie, die WIERZBICKA für den Gebrauch der englischen Verben to build und to cook feststellt, sofern diese in Sätzen verwendet werden, in denen die durch die Verben bezeichneten Handlungen so dargestellt werden, als ob sie nichtintentional vollzogen wüden (s.o.), gilt ebenfalls für deren russische Aquivalente stroit, und varit, (in der Bedeutung čto-libo iz čego-libo, 2.B. sup iz ryby) sowie für sämtliche anderen faktitiven Verben des Russischen; vgl. die semantisch nichtwohlgeformten Sätze (29) und (30) mit den Verben stroit', vjazat', varit' und peǒo:

(29) Boris nenamerenno postroil garaž/svjazal pulover. (30) Nataša suarila sup/ispekla pirog po nevnimatel'nosti. Paradigmatisch für deren lexikalische Bedeutungen ist die intentionale Einbezogenheit des Agens in die Handlung auch bei einer Gruppe von kausativen Verben, die im Vergleich zur Klasse der faktitiven Verben semantisch nicht so homogen ist. Bei diesen Lexemen handelt es sich um Verben, die Tatbestände bezeichnen, die Sprecher in der Regel als juristisch kriminalisiert bzw. juristisch kriminalisierbar ansehen, wie beispielsweise krast', pochisčat', vzlamyvat', vorovat', santažirovat' iznasilovat', uvol'njat', bastovat' zaverjat'/udostoverjat' (in der Bedeutung von beglaubigen z.B. zaverjat' dokument). 20 zu dieser Gruppe können noch einige kausative Verben gerechnet werden, die Handlungen bezeichnen, welche Sprecher in der Regel als moralisch verwerfbar einstufen, wie beispielsweise utaivat', obmanyvat' lgat', vydavat' sebja za kogo-libo (Z.B. vydavat' sebja za vrača ), uprekat', ukorjat'. Das gemeinsame Klassenmerkmal der lexikalischen Bedeutungen dieser Verben besteht darin, daB sie bestimmte Formen menschlichen Verhaltens aus einem Bereich bezeichnen, der, bedingt durch das gesellschaftioche Interesse, die entsprechenden Handlungen mit positiven bzw. negativen Sanktionen zu belegen, begrifflich in hohem MaBe kodifiziert ist. Die eindeutige Referenz, die mit Hil- 
fe der lexikalischen. Bedeutungen der oben genannten Verben auf die intentionale Einbezogenheit des Agens in die Handlung hergestellt wird, verbietet es daher, diese Denotationsbedingung auBer Kraft zu setzen und Verben wie beispielsweise pochišcat' und uprekat' zur Bezeichnung einer nichtintentionalen Handlung zu verwenden; vgl. die semantisch nichtwohlgeformten Sätze (31) - (32):

(31)* Ital'janskie bandity nenamerenno pochitili detej nemeckogo žurnalista.

(32) Agenstvo TASS po nevnimatel'nosti upreknulo amerikanskich žunalistov $v$ propagande.

Nicht nur die Vollständigkeit, die die Konstrukteure des MST von den Bedeutungsexplikationen des EKW fordern, wird durch die Vernachlässigung der Intentionalität kausierter Handlungen in bestimmten Fällen beeinträchtigt. Negative Folgen ergeben sich in den gleichen Fällen auch für die Modellierung synonymischer Paraphrasebeziehungen zwischen Texten, die das MST erklärtermaßen beschreiben soll (vgl. S. $6 \mathrm{ff}$. ). Die Fähigkeit von Sprechern, den Sätzen (33) und (34) die implikativen Paraphrasen oder semantischen Schliusse (33') und (34') zuzuordnen, können die Konstrukteure des MST nicht erfassen:

(33) Rabociie gorodskogo transporta bastujut.

(33') Rabočie gorodskogo transporta namerenno/s umyslom prekratili rabotu.

(34) Mestnye vlasti utaili padenie samolëta.

(34') Mestnye viasti namerenno/s umyslom ne soobšcili o padenii samolëta.

Die Weigerung der Konstrukteure des MST, ein Sem 'namerenno' in die Beschreibung solcher kausativen Verben aufzunehmen, fur die es sich dabei um eine paradigmatische Eigenschaft handelt, steht auch im Widerspruch zu ihrer Behandlung bestimmter nomina agentis (imena dejatelja). Einige Substantive, die sie mit Hilfe der lexikalischen Funktion $S_{1}$ als nomina agentis von den entsprechenden Verben ableiten (vgl. S. lly ff.), spezifizieren die Konstrukteure des MST durch die Zusätze 'okkasionell' ('aktual'noe') bzw. 'habituell' ('abi- 
tual'noe'):

"... einige russische nomina agentis... bezeichnen nur einen habituellen Agens (povar, vrać, sofër lund naezdnik; K.H.J, andere sowohl einen habituetTen als auch einen okkasionellen (igrok, plovec, lyzinik) und solche wie voadnik (prochozij u.a.) nur einen okkasionellen Agens (diese Dichotomie wird auch häufig mit der Unterscheidung 'professioneller/nichtprofessioneller Agens' belegt; vgl. rybak - rybolov)." (ZOLKOVSKIJ/MEL' CUK 1969, 30-31)

Es ist nicht einzusehen, warum die Konstrukteure des MST es nicht fur erforderlich halten, in die Bedeutungsexplikationen der oben genannten Kausativa eine Bedeutungskomponente 'namerenno' aufzunehmen, während sie die Bedeutungen bestimmter nomina agentis durch die Spezifizierung 'aktual'noe' bzw. 'abitual'noe' disambiguieren. Da die intentionale Einbezogenheit des Agens in die Handlung für die lexikalischen Bedeutungen der oben genannten kausativen Verben paradigmatisch ist, muB in ihren Explikationen das Sem 'kauzirovat'. durch ein attributiv mit inm verknüptes Sem 'namerenno' modifiziert werden; vgl. die von uns erstellte und gemäB APRESJAN $1974 \mathrm{~b}$ wortsprachlich und formal notierte lexikographische Definition (35) des Verbs krast':

(35) A kradët $B-u \quad C-a=$ 'A namerenno kauziruet $C$ perestavat' imet' $B$.'

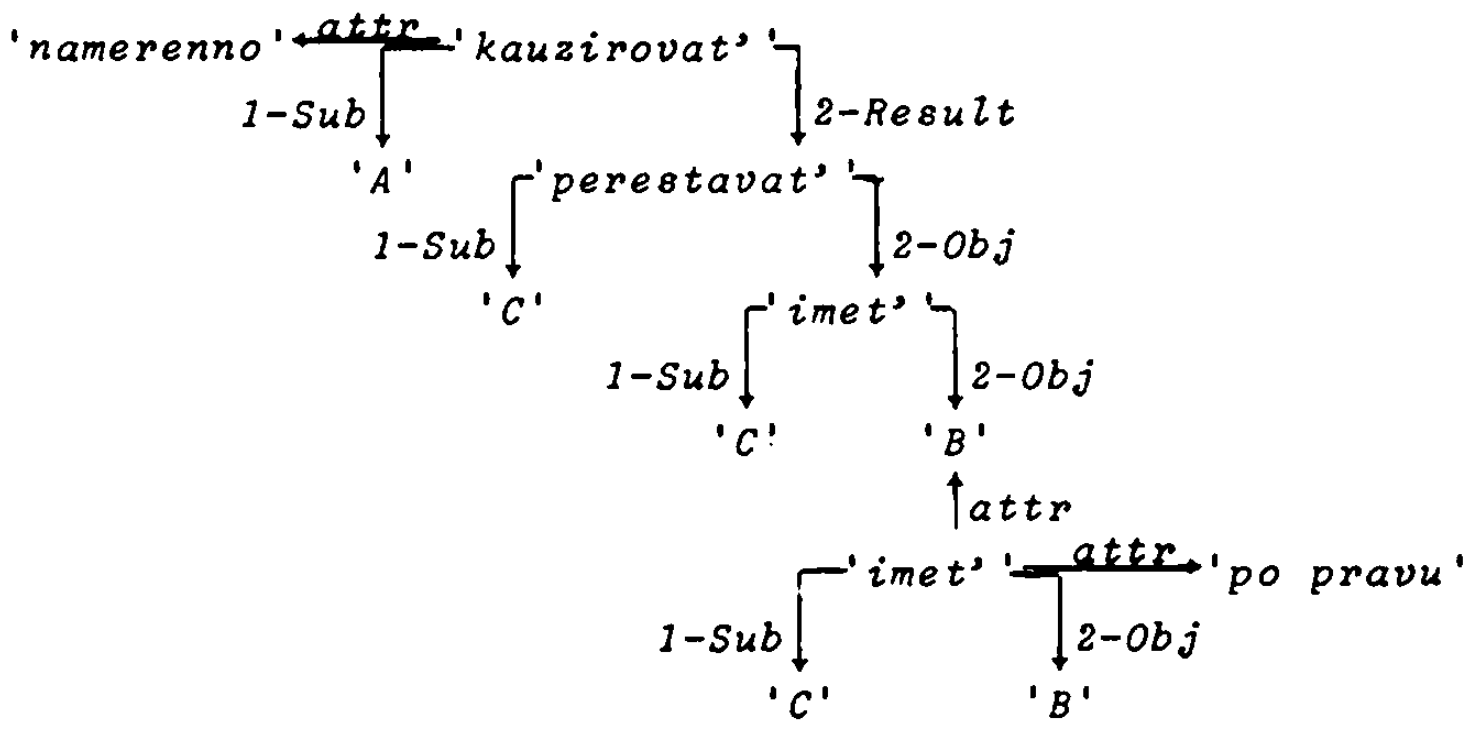

Das Vorgehen der Konstrukteure des MST, das Problem der Intentionalität kausierter Handlungen generell in die parole abzuschieben, beeinträchtigt, wie oben gezeigt, die zielset- 
zungen des Modells in bestimmten Fällen ganz erheblich. Bei den oben genannten kausativen Verben muB die intentionale Einbezogenheit des Agens in die Handlung daher unbedingt auf der Abstraktionsebene der langue beschrieben werden.

Die Forderung, daB zwischen Inhalt und Ausdruck der Einheiten der Sems eine ein-eindeutige Beziehung zu bestehen habe, lösen die Konstrukteure des MST auch bei der Verwendung bestimmter syntaktischer Ausdrucksmittel der Sems nicht immer ein. In der wortsprachlichen Notationsvariante der Sems benutzen sie synonymische grammatische und lexikalische Ausdrucksmittel, um eine attributive Verknüpfung zwischen Bedeutungskomponenten einer lexikographischen Definition zu signalisieren. Einige dieser Ausdrucksmittel sind in den Bedeutungsexplikationen (36) - (40) der Stichwörter echat', nescast'e, upuskat' izvidu, drobit' und lovkij in einer ihrer Bedeutungen enthalten:

(36) A edet iz Y-a $v 2$ na $W-e=$ 'A pereměsčaetsja iz $Y-a v$ 2 , potomu čto A nachoditsja na $N-e$, kotoroe peremeščaetsja iz $y-a v 2$, $i$ peremešcenie iz $Y-a v z$ vchodit $v$ čislo celej $A-a$.'

(37) $A-\underline{\text { nesčast'e }}=$ ' $\chi$ - sobytie, okazyvajuščee otricatel'noe ili pagubnoe vlijanie na normal'nuju ziznedejatel'nost' čeloveka.'

(38) y upuskaet iz vidu $x=$ 'Y dejstvuet, ne znaja, čto $x$ sušcestvenen dija dejatel'nosti $y-a . '$

(39) $A$ drobit $B X-o m={ }^{\prime} A$ delit tvërdyj i pročnyj $B$ udarom instrumenta $X$.'

(40) $x$ - $\underline{\text { lovkijj }}=$ ' $x$-obladajuščij sposobnost'ju bystro $i$ bez truda koordinirovat' dvizenija svoego tela pri vypolnenii fizičeskich dejstvij.' (APRESJAN 1974b, 107, 108, 109)

Während in (36), (37) und (38) die grammatischen Ausdrucksmittel Relativpronomen ('...na $w-e$, kotoroe ...'), Partizip 
('...sobytie, okazyvajuščee ...') und Gerundium ('... dejstvuet, ne znaja ...') eine attributive Verknuipfung zwischen Bedeutungskomponenten anzeigen, ${ }^{21}$ erfüllen diese Funktion in (39) und (40) die lexikalischen Ausdrucksmittel 'udarom' und 'pri vypolnenii'. Dem Ausdruck 'pri vypolnenii' kommt dabei neben der attributiven Verknuipfung der beiden Teilexplikationen die Aufgabe zu, die temporale Bedeutungskomponente 'Gleichzeitigkeit' zu signalisieren. In ähnlicher Weise ist der Ausdruck 'udarom' funktional belastet.

Die lexikographischen Definitionen (36) - (40) zeigen, daB die Konstrukteure des MST in der wortsprachlichen (= lexikographischen) Notationsvariante der SemS die Reglementierung ihrer Ausdrucksmittel weitgehend aufgeben. Den Grund, aus dem sie in den lexikographischen Definitionen des EKW verschiedene Ausdrucksmittel einsetzen, iber die das Russische verfügt, um attributive Verknüpfungen zu signalisieren, erörtern die Konstrukteure des MST nicht. Es dijrfte jedoch in dem Bestreben liegen, die Bedeutungsexplikationen für die potentiellen Adressaten des Wörterbuchs so verständlich wie möglich zu machen. Zweifellos ist die Verstehbarkeit der lexikographischen Definitionen des EKW eher gewährleistet, wenn in ihren Texten nicht nur weitgehend grammatisch richtiges, sondern auch idiomatisches Russisches verwendet wird (vgl. allerdings auch S. 140).

Im Zusammenhang mit den Relationen der Attribution und der Koordination, die in der Syntax der Sems vorgesehen sind, muB den Konstrukteuren des MST auch der Vorwurf gemacht werden, daB sie ihre Verwendung bei der semantischen Beschreibung des russischen Wortschatzes nicht erläutern. Unsere Kritik gilt allerdings nur fü die (wortsprachlich oder formal notierte) lexikographische Variante der SemS des EKW. Für die semS des MST, die sich von der des EKW erheblich unterscheidet (vgl.s.9 ff.), stellt sich das hier in Rede stehende Problem nicht. MEL'CUK setzt auf dieser Abstraktionsebene als einzige syntaktische Relation die Prädikat-Argument-Relation an. Sämtliche Strukturen zum Ausdruck von Oberflächenattributionen werden daher auf prädikative Relationen dieses Typs $z u-$ rückgeführt (MEL'CUK 1974b,62-64; 1974C II, 34). 
Für die lexikographische Variante der SemS des EKW entsteht jedoch der Verdacht, daB die Konstrukteure des MST in bestimmten Fällen die attributive und die koordinative Verknüpfung synonymisch, d.h. zu Bezeichnung der gleichen semantischen Relation zwischen Bedeutungskomponenten einsetzen; vgl. die lexikographischen Definitionen (41) - (42) von prichodit' und privodit':

(41) A prichodit $v B=$

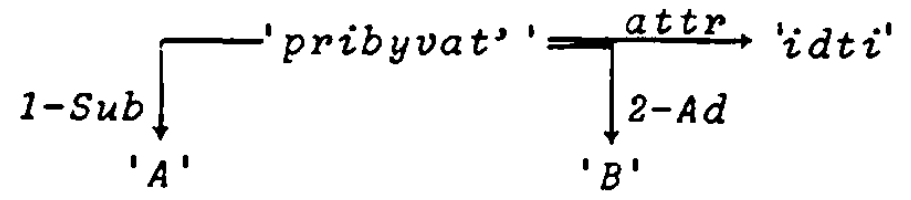

(42)

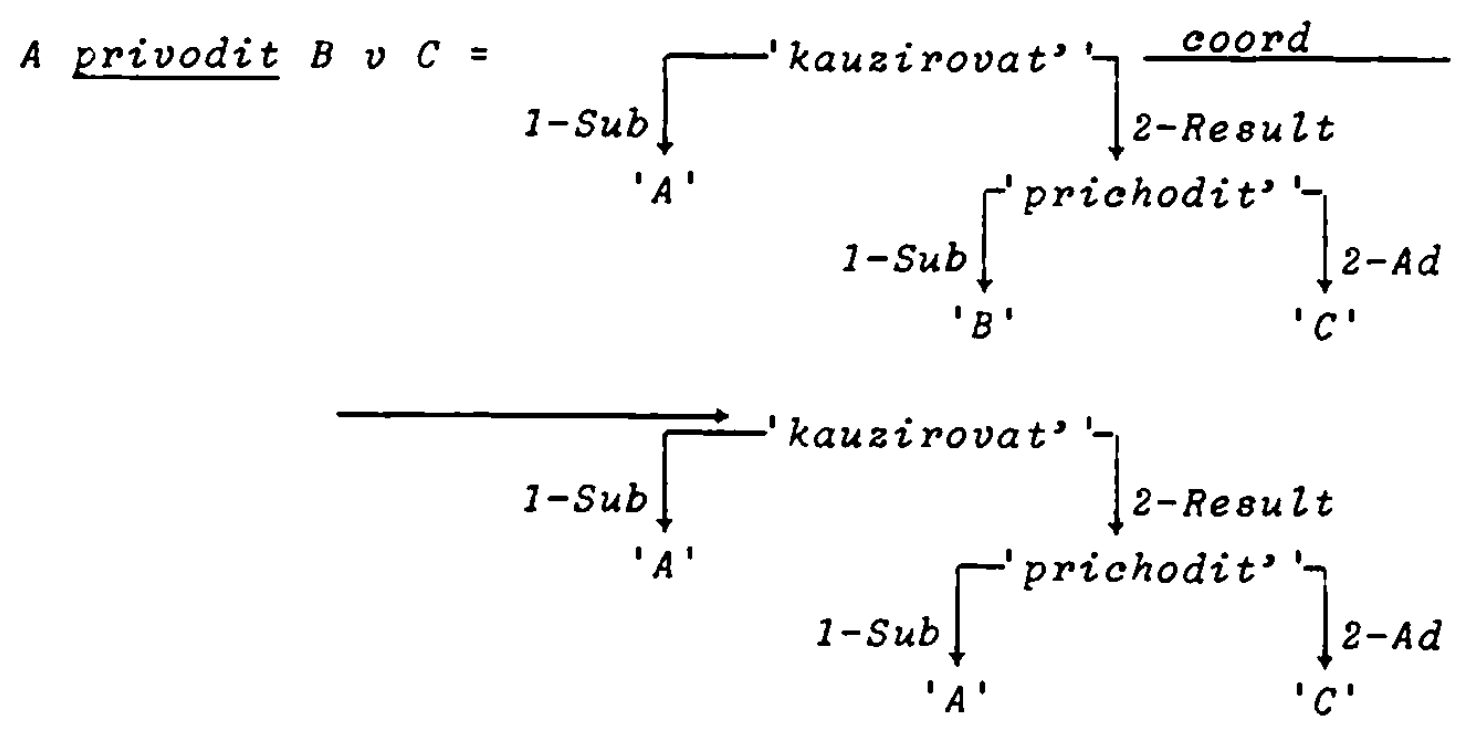

(APRESJAN 1969c, 422)

Es ist nicht einzusehen, warum die semantische Relation in (41) zwischen den beiden attributiv verknüpften Semkomplexen eine andere sein soll als die zwischen den beiden koordinativ verknüpften Semkomplexen in (42). Auf den S. $161 \mathrm{ff}$. unternehmen wir - allerdings unter einer anderen als der hier besprochenen Fragestellung - den Versuch, eine Verwendungsweise der Attributions- und der Koordinationsrelation festzulegen, die dem Postulat der Synonymie- und Homonymiefreiheit, das die Konstrukteure des MST an die SemS richten (vgl. S. $39 \mathrm{ff}$.), entspricht. 
2.2.1.2 Die lexikographische Definition im erklärend-kombinatorischen Wörterbuch

Im EKW erfolgt die lexikographische Definition mit Hilfe der SemS. Bei der Explikation von Wortbedeutungen wenden die Konstrukteure des MST das Verfahren der lexikalischen Dekomposition (Zeksičeskoe razloženie) an. Sie begreifen lexikalische Bedeutungen als Erscheinungen mit einer inneren syntaktischen Struktur:

"Sogar die Bedeutung von Einzelwörtern hat eine komplexe innere Organisation, die auf jeden Fall nicht weniger komplex ist als die Organisation eines Satzes: iubrigens ist es kein Zufall, daß Wortbedeutungen häufig mit $\mathrm{Hilfe}$ eines oder mehrerer Sätze expliziert werden." (MEL'CUK 1974b, 61)

Das Verfahren der lexikalischen Dekomposition erlaubt es, mit Hilfe syntaktischer Mittel Beziehungen zwischen den Bedeutungskomponenten eines zu explizierenden Lexems anzugeben. Beispielsweise kann in der Bedeutungsexplikation von krast' (vgl. S. 59) durch die Dependenzrelation, die die Gegenstandsvariable ' $A$ ' in der Agensrolle mit dem Prädikat 'kauzirovat" verknupft, und die Attributionsrelation, die 'kauzirovat' durch das Sem 'namerenno' modifiziert, angegeben werden, daB beide Bedeutungskomponenten begrifflich zusammengehören, da das Verb eine intentional kausierte Handlung bezeichnet. Das Verfahren der lexikalischen Dekomposition ist dem der Merkmalsanalyse lexikalischer Bedeutungen ubberlegen, denn es kann Wortbedeutungen als komplex strukturierte Gebilde beschreiben, deren einzelne Bestandteile unterschiedlichen semiotischen Typen zugeordnet sind, die durch verschiedene Relationen miteinander verbunden werden (MEL'CUK 1974b, 62). Im Gegensatz zur lexikalischen Dekomposition können in der Merkmalsanalyse lexikalische Bedeutungen nur mit $\mathrm{Hilfe}$ unstrukturierter Mengen semantischer Merkmale 22 oder durch nur relativ gering strukturierte Konstellationen aus semantischen Merkmalen (BIERWISCH 1970) beschrieben werden. Daher hat sich die semantische Merkmalsanalyse auch bisher vornehmlich mit der Untersuchung von Wortschatzbereichen befaBt, deren Einheiten wie beispiels- 
weise Verwandtschafts- und bestimmte Gattungsbezeichnungen konkrete Referenten bezeichnen, und den Verbalbereich fast völlig ausgeklammert. Der Grund für eine derartige Einschränkung des Gegenstandsbereichs der lexikalischen Semantik liegt darin, daß den Bedeutungen der Lexeme dieser Wortschatzausschnitte eine begrifflich wenig komplexe struktur zugewiesen werden kann.

Indem sie am Beschreibungsinstrumentarium der Merkmalsanalyse festhält, unterwirft die linguistische Semantik jedoch ihren Forschungsgegenstand erheblichen Beschränkungen. Dieser Umstand veranlaßt die Konstrukteure des MST zu der Forderung, in der linguistischen Semantik die Vorstellung von der lexikalischen Bedeutung als einer unstrukturierten Menge von Bedeutungskomponenten aufzugeben:

"... bis heute werden in vielen Semantiktheorien Bedeutungselemente - analog den distinktiven Merkmalen der Phoneme als homogen (wie beispielsweise in der Theorie von KATZ und FODOR) angesehen; das zeigt ganz einfach, daB die Strukturiertheit, die innere Syntax semantischer Repräsentationen nicht anerkannt wird." (MEL'CUK 1974b, 62)

Die lexikographische Definition macht im wesentlichen die Eigenschaften des EKW aus, ein definitorisches oder erklärendes Wörterbuch zu sein. Einen Beitrag zur Beschreibung der Wortbedeutung leistet auch der illustrative Teil, den jeder Wörterbuchartikel des EKW umfaßt (vgl. S. 72 ff.), sowie die konnotative Zone und der modale Rahmen, die bei Bedarf in den Wörterbuchartikel aufgenommen werden (vgl. S. 97 ff.).

Bei der Explikation von Wortbedeutungen gehen die Konstrukteure des MST introspektiv vor (vgl. S. 3s ff.). Um inre sprachliche Vorstellungskraft möglichst stark zu mobilisieren, bedienen sie sich der folgenden vier Verfahren, die von ZOLKOVSKIJ unter dem Begriff "Porträtierung des Wortes" (portretirovanie slova; ZOLKOVSKIJ 1964, 9) zusammengefaBt werden (APRESJAN 1974b, 99-106):

1) Sie konstruieren die durch das Stichwort typischerweise bezeichnete auBersprachliche Situation, indem sie sämtliche beteiligten Mitspieler mit ihren semantischen Eigenschaften und Handlungen aufzählen. 
2) Sie explizieren die Bedeutung eines Lexems in seiner Satz-/ oder Situationsform (sentencionnaja forma, situacionnaja forma; MEL'CUK 1974b, 111). d.h. Sie analysieren das Wort auf der syntagmatischen Achse in einem Satzkontext, der sämtliche Mitspieler der durch das Wort typischerweise bezeichneten Situation als Gegenstandsvariable enthält.

3) Sie vergleichen paradigmatisch verbundene Wortgruppen, um ihre Bedeutungsunterschiede und -gemeinsamkeiten erfassen zu können.

4) Sie untersuchen sogenanntes negatives sprachliches Material, d.h. sie konstruieren semantisch nichtwohlgeformte Sätze und versuchen, über den Grund des VerstoBes AufschluB über die lexikalische Bedeutung des zu explizierenden Lexems zu gewinnen.

Die Konstrukteure des MST scheinen sich - wie auch andere Wörterbuchautoren - uberhaupt nicht die Frage zu stellen, ob es für die lexikographische Praxis sinnvoll ist, in den Wörterbuchartikeln des EXW nicht nur Lexemkollokationen, sondern auch Explikationen von Wortbedeutungen zu berücksichtigen. 23 Das Bedurfnis von Sprechern, Definitionslexika zu befragen, um sich uber die Bedeutungen der von ihnen explizierten wörter zu informieren, die keineswegs zu Spezialwortschätzen zu rechnen sind, setzen sie einfach voraus. In diesem Sinne reint sich das EKW zumindest hinsichtlich seiner Zweckbestimmung als Definitionslexikon nahtlos in die Serie einsprachiger erklärender Wörterbücher ein, die zwar in unserem Kulturkreis in hoher Auflagenzahl verbreitet sind. von denen jedoch bisher niemand genau festgestellt hat, ob ihre Benutzer sie wirklich wegen einer Wortdefinition oder vielleicht nur wegen einer Orthographie, Flexionsangabe oder stilistisch wohlgefarmter Wortverbindung befragen. In änlicher Weise stellt auch WEINREICH die Frage nach dem Zweck, dem nichtfachsprachliche einsprachige Definitionswörterbücher in der praktischen Lexikographie dienen sollen:

"Die Tatsache, daB in unserer seltsamen Kultur eine hohe Nachfrage nach einsprachigen Definitionslexika besteht, die 
Definitionen solcher. Wörter enthalten, die so bekannt sinc, daB wahrscheinlich niemand den Wunsch hat, sie nachzuschligen, ist an sich schon ein interessanter ethnographischer Sachverhalt." (WEINREICH 1967, 26-27)

Auch wir vermögen nicht zu sagen, ob Sprecher in nichtfachsprachlichen einsprachigen Definitionswörterbichern wirklich Explikationen von Wortbedeutungen nachschlagen. Allerdings glauben wir, daB bestimmte Forderungen, die dif Konstrukteure des MST an die Form der lexikographischen DEfinition im EKW stellen, relativiert werden mussen (s.u.), da die Berechtigung, mit der sie den Anspruch erheben, das Bedurfnis potentieller Wörterbuchbenutzer gerade nach Definitionen usueller Wörter zu erfillen, aus der Sicht der lexikographischen Praxis nicht ohne weiteres abgesichert ist. 24

Die Konstrukteure des MST formulieren drei Bedingungen, denen die lexikographischen Definitionen im EKW genuigen muissen:

1) Die lexikographischen Definitionen des EKW sollen die Eedeutungen der zu explizierenden stichwörter nicht direkt in elementare Bedeutungen zerlegen, sondern ihre Dekomfosition soll schrittweise uber Lexeme mit abnehmender stmantischer Komplexität erfolgen:

"Bei jedem Schritt der Dekomposition müssen die größter semantischen Konstituenten der zu explizierenden Bedeutung herausgearbeitet werden ..." (APRESJAN 1968, 36)

"Die Definition soll einen unbekannten Begriff auf bereits bekannte reduzieren. Für die Lexikographie ist es offensichtlich ausreichend, wenn man fordert, daB eine komplexe Bedeutung durch einfache Bedeutungen, ihre Korstituenten, bis hin zu elementaren Konstituenten expliziert wird." (APRESJAN 1969a, 12)

"Die zu explizierende Bedeutung ' $A$ ' muß in möglichst groBe semantische Konstituenten zerlegt werden, bei denen es sich folglich nicht nur um elementare Bedeutungen, sondern auch um Wörter der erweiterten semantischen Sprache handeln kann." (APRESJAN 1969c, 421)

"Das zu explizierende Wort muB durch einfachere Bedeutungen expliziert werden. Zugleich muB es letztlich auf eine kleine Anzahl elementarer (undefinierter) Bedeutungen - die Wörter der semantischen Sprache - reduziert werden ..." (APRESJAN 1974b, 95) 
"Keine Bedeutungsexplikation soll direkt aus den einfachsten, sondern aus den höchstmöglich komplexen Einheiten, die im Wörterbuch enthalten sind, d.h. aus'semantischen unmittelbaren Konstituenten' (aus Wörtern, die ihrerseits selbst an entsprechender stelle expliziert werden) konstruiert werden. M.a.W., wenn $A$ durch die Basiswörter $B_{1}, B_{2}, B_{3}, B_{4}, B_{5}$ definiert werden kann, es aber in der gegebenen Sprache die Wörter $C=B_{1}+B_{2}$ $D=B_{3}+B_{4}+B_{5}$ gibt, so muB $A$ durch $C$ und $D$ expliziert werden." (MEL'CUK 1974b, 111)

2) Die lexikographischen Definitionen des EKW sollen die Bedeutung des zu explizierenden Stichworts vollständig und ohne Redundanz beschreiben:

"Die elementaren semantischen Merkmale muissen derart sein, da $B$ zwei beliebige, intuitiv verschiedene Bedeutungen formal unterschieden und zwei beliebige, intuitiv gleiche Bedeutungen formal identifiziert werden können." (APRESJAN 1968,38 )

"Die Bedeutungen des zu definierenden und des definierenden Ausdrucks sollen gleich sein; wenn $A$ das Definiendum ist und ' $B C$ ' das Definiens, so mussen die Folgerungen $A \rightarrow B C$ und $B C \rightarrow A$ gelten, d.h. die Bedingung, da $B$ die Merkmale ' $B C$ ' für $A$ notwendig und hinreichend sind." (APRESJAN 1969a, 12)

"Fuir eine zu definierende Bedeutung ' $A$ ' muissen die definierenden Bedeutungen ' $B C$ ' notwendig und hinreichend sein (es missen alle semantischen Komponenten von $A$ und nur diese aufgezählt werden; die Definition muB die genaue synonymische Paraphrase des Definiendum sein)." (APRESJAN $1974 b, 95)$

"Das zu explizierende Nort und seine Explikation mussen bedeutungsgleich sein, d.h., daB alle Elemente der Explikation notwendig und hinreichend sein müssen. Die Bedeutungsexplikationen garantieren dann eine formale Unterscheidung zweier beliebiger, intuitiv verschiedener Wörter und die Identifizierung zweier beliebiger, intuitiv synonymischer Wörter." (MEL'CUK 1974, 111)

"... sprachlichen Ausdrücken, die intuitiv nicht bedeutungsgleich sind, mussen verschiedene Bedeutungsbeschreibungen zugewiesen werden .... sprachliche Ausdrücke, die intuitiv bedeutungsgleich sind, missen identische Beschreibungen enthalten ... Unterschiede, die sich in Wortpaaren regulär wiederholen, d.h., die intuitiv als gleich empfunden werden, müssen einen identischen formalen Ausdruck in der Bedeutungsrepräsentation erhalten." (MEL'CUK 1974b, 75) 
3) Die lexikographischen Definitionen des EKW dirfen keine tautologischen Zirkel enthalten:

"Die Definitionen dürfen nicht zirkulär sein, d.h. A sollte nicht durch $B$ und $B$ nicht durch $A$ definiert werden (wie es in ožegovs Wörterbuch der russischen Sprache und anderen gängigen Wörterbüchern der Fall ist) ... (APRESJAN 1969b, 4)

"In der Bedeutungsexplikation darf es keinen tautologischen Zirkel geben..." (APRESJAN 1969c, 421)

"In der Bedeutungsexplikation darf es keinen Definitionszirkel geben, d.h. eine Explikation von $A$ durch $B$ und $B$ durch $A$. Alle Bedeutungsexplikationen werden letztlich auf eine Kombination einfachster (undefinierter) Bedeutungselemente, d.h. Basiswörter oder $\mathrm{S} e \mathrm{~m} e$, reduziert." (MEL'CUK 1974b, 111)

Wir wollen die oben genannten drei Bedingungen, denen die Konstruktuere des MST die lexikographischen Definitionen des EKW unterwerfen, nacheinander kurz kommentieren, um die Aufgaben zu erläutern, die ihnen bei der semantischen Beschreibung des russischen Wortschatzes zufallen:

Zu 1):

Durch die Forderung an die lexikographischen Definitionen des EKW, die Bedeutungen der zu explizierenden Stichwörter hierarchisch und schrittweise in Kombinationen aus einfacheren Bedeutungen zu zerlegen, unterstreichen die Konstrukteure des MST den strukturellen Charakter ihrer semantischen Beschreibung. Sie beabsichtigen, den russischen Wortschatz zu strukturieren, indem sie vermittels der lexikographischen Definitionen des EKW Bedeutungsrelationen zwischen bestimmten lexikalischen Einheiten angeben. Zu diesem Zweck bedienen sie sich des Konzepts des erweiterten Lexikons (vgl. S. $20 \mathrm{ff.}$ ), demzufolge Wörter, die bereits expliziert worden sind, ihrerseits zur Definition anderer Wörter verwendet werden können. Auf diese Weise beschreiben die Konstrukteure des MST u.a. mit Hilfe von einseitigen Inklusionsbeziehungen zwischen bestimmten lexikographischen Definitionen die Bedeutungsrelationen zwischen den entsprechenden Stichwörtern. In eine semantische Relation werden beispielsweise die Le- 
xeme izmenjat', izmenjat'sja, stanovit'sja und inoj gesetzt, da ihnen im EKW lexikographische Definitionen zugewiesen werden, aus denen hervorgeht, daB die Bedeutungen von inoj und stanovit'sja in der Bedeutung von izmenjat'sja und in der Bedeutung von izmenjat, enthalten sind (die gesperrt gedruckten Seme werden als elementar, d.h. als nicht weiter zerlegbar angesehen):

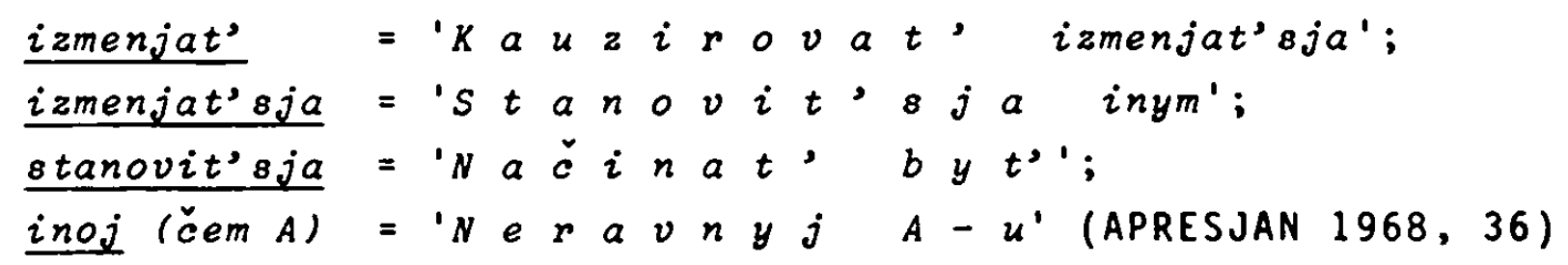

Eine hierarchische Inklusionsbeziehung setzen die Konstrukteure des MST auch zwischen den Bedeutungen der Wörter uničtǒ̌at', iš̌cezat' und perestavat' an:

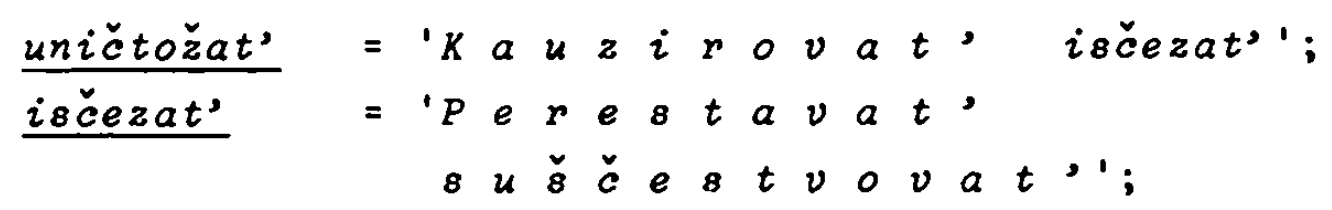

Die Konstrukteure des MST verweisen darauf, daß gängige Definitionswörterbücher des Russischen ein ähnliches Explikationsverfahren benutzen ( $v g l$. S. $152 \mathrm{f.}$ ), das jedoch nur in seltenen Fällen ein Stichwort auch wirklich in seine einfacheren Bestandteile dekomponiert. Explikationen des Typs arestovyvat' $=$ 'Podvergat' arestu', besedovat' $=$ 'Vesti besedu', $\underline{\text { kritikovat' }}=$ 'Podvergat' kritike' und pomogat' $=$ 'Okazyvat' pomošč", die in diesen Wörterbüchern in grober Anzahl zu finden sind, zerlegen die Bedeutungen der entsprechenden Stichwörter nicht, da die Verben in den oben genannten Explikationen als bedeutungsleer angesehen werden können und dem kombinatorischen Potential der jeweiligen Substantive zugewiesen werden können (vgl. S. $119 \mathrm{ff.}$ ):

"Die Wörter podvergat", vesti $u . \ddot{a}$. haben in den oben genannten Fällen keine selbständige Bedeutung. Ihrem Wesen nach erfillen sie die rein syntaktische Hilfsfunktion eines verbbildenden Morphems." (APRESJAN 1969a, 14)

Das Verfahren, bei der lexikographischen Definition im EKW die Bedeutung eines Stichworts nicht direkt, sondern 
schrittweise auf seine elementaren Komponenten zurijckzuführen, bringt den unbestreitbaren Vorteil mit sich, die Bedeutungsexplikationen des Wörterbuchs für seine - wie auch immer definierten - potentiellen Benutzer überschaubarer und leichter zugänglich gestalten zu können. AuBerdem ermöglichst es die schrittweise Dekomposition von Wortbedeutungen, bestimmte semantische Relationen auszunutzen, um die Einheiten eines Wortschatzes im Wörterbuch systematisch anzuordnen.

Zu 2):

Hinter der Forderung, die die Konstrukteure des MST an die lexikographischen Definitionen des EKW richten, die Bedeutung des Explikandum redundanzfrei zu explizieren, steht das Bemuihen, bei der semantischen Beschreibung möglichst ökonomisch vorzugehen.

Das Postulat nach Vollständigkeit der lexikographischen Definition ergibt sich einerseits - wie die unter 1) genannte Forderung - aus der strukturellen Beschreibung der Lexik im EKW. Die Explikationen des Wörterbuchs sind dann vollständig, wenn sie auf explizite Weise, d.h. mit formalen Mittelr, die intuitiv ermittelten Bedeutungsgleichheiten und - unter schiede zwischen den Einheiten eines Wortschatzes angeben. Wird dem semantischen Unterschied, der beispielsweise zwischen den Verben prekrašcat, und končat' hinsichtlich der Art und Weise besteht, in der die durch beide Lexeme bezeichnete Handlung beendet wird (s.u.), nicht durch eine formale Unterscheidung der Bedeutungsexplikationen, die man innen zuweist, Rechnung getragen, so ergibt sich eine falsche Synonymiebeziehung zwischen beiden Lexemen; vgl. die Paraphrase (1):

(1) Maljary prekratili rabotu. - Maljary končili rabotu. Um (annähernd) vollständig zu sein, müssen die lexikographischen Definitionen der beiden oben genannten Verben ergänzt werden. In die Bedeutungsexplikation von končat' muB der Hinweis aufgenommen werden, daB die Handlung, die eingestellt wird, bis zu einer naturlichen Grenze durchgeführt worden ist; die Bedeutungsexplikation von prekraščat' muB 
durch den Hinweis ergänzt werden, daB die Einstellung der Handlung zu jedem beliebigen Zeitpunkt, jedoch immer bevor sie zur Erreichung eines $Z$ iels gefïht hat, erfolgen kann (APRESJAN 1974b, 96).

In ähnlicher Weise muB durch explizite Zusätze in den Bedeutungsexplikationen der semantische Unterschied angegeben werden, der beispielsweise zwischen den Verben davat' und soobšcat' sowie poterjat, und zabyvat, bezijglich der möglichen lexikalischen Besetzungen ihrer objektergänzungen besteht; vgl. die Definitionen (2) - (5):

(2) davat' = 'Eksplicitno kauzirovat' imet' material'nyj ob-ekt ili ènergiju';

(3) $\underline{800 b \dot{B} c a t '}=$ 'Éksplicitno kauzirovat' imet' informaciju $o^{\prime}$;

(4) poterjat' = 'Nenaročno perestavat' imet' material'nyj ob-ekt ili ènergiju';

(5) $\underline{\text { zabyvat' }}=$ 'Nenaročno perestavat' imet' informaciju 0 ' (MEL'CUK 1974b, 75) 25

Da der Bedeutungsunterschied zwischen den Lexemen beider Wortpare von den Konstrukteuren des MST als gleich empfunden wird, weil sich in ihnen die Opposition 'material'nyj ob-ekt $i l i$ energija' vs. 'informacija' regulär wiederholt, stellen sie die vier Lexeme in einer semantischen Proportion dar; vgl. (6):

(6) davat' $^{\text {soobšcat' }}=$ poterjat' $: \underline{z a b y v a t^{\prime}}$

Das Postulat der Vollständigkeit der lexikographischen Definition ergibt sich andererseits aus dem Bestreben der Konstrukteure des MST, die Bedeutungen lexikalischer Einheiten so ausführlich zu beschreiben, daB die invarianten, von allen Mitgliedern einer Sprachgemeinschaft vergesellschafteten 8 egriffe erfaBt werden können, die die Sprecher mit diesen Einheiten verbinden. Es ist selbstrerständich, daB die Probleme, auf die man bei dem Versuch stöBt, lexikalische Bedeutungen als überindividuelle Begriffe zu beschreiben, es nur erlauben, eine approximative Vollstänigkeit von lexikographischen Definitionen zu erzielen. 
Vor allem aufgrund der individuellen, d.h. der sozio- und idiolektalen Variationen, denen Wortbedeutungen unterliegen können, kommt man in der (synchronen) lexikalischen semantik nicht umhin, die Vollständigkeit der Bedeutungsexplikationen als einen Idealfall zu betrachten (MEL'CUK $1974 b, 111)$.

Die Konstrukteure des MST versuchen zumindest in einigen Wörterbuchartikeln des EKW durch zusätzliche Einträge die Unzulänglichkeiten auszugleichen, die das von ihnen verwendete metasprachlich-reduktionistische Verfahren der lexikographischen Definition für die vollständige Erfassung der Wortbedeutung in sich birgt. In der form eines speziellen illustrativen Teils (ilzjustrativnaja čast') und eines speziellen kontrastiven Teils (sopostavitel'naja čast') nehmen sie in bestimmte Wörterbuchartikel zwei weitere Bereiche auf. In ihnen finden sich als Beispiel fïr verschiedene, jedoch innerhalb derselben Bedeutung angesiedelte Verwendungsweisen des Stichworts mehrere Säze bzw. andere Lexeme, die zu dem Stichwort in der Relation der Bedeutungsnähe stehen. Beispielsweise enthält der illustrative Teil des Wörterbuchartikels für arestovyvat, die folgenden Sätze: Policija arestovala prestupnika prjamo na bortu samolëta. On byl arestovan pri popytke perejti granicu. 6 marta patriot popal $v$ ruki kondutovskich satrapov, no čerez tri dnja byl osvoboždën pod davleniem obščstvennosti. (MEL'CUK $1974 b, 117$ )

Im kontrastiven Teil dieses Wörterbuchartikels sind die mit arestovyvat, bedeutungsähnlichen verben bzw. Wortverbindun-

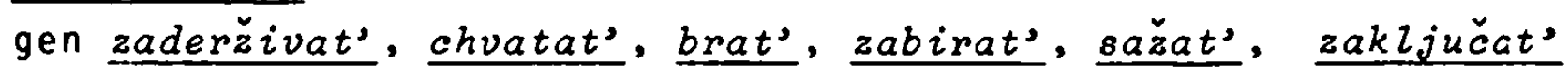
b tjur'mu, brat' $v$ plen und internirovat' samt ihrer lexikographischen Definitionen aufgelistet (MEL'CUK 1974b, ebd.).

Fordern die Konstrukteure des MST von den Bedeutungsexplikationen des EKW Vollständigkeit, so sind sie gezwungen (im Idealfall), all diejenigen Seme anzugeben, die für das Explikandum als Denotationsbedingungen in Frage kommen können. Semantische Komponenten, die, um die Potenz eines Wortes zur Referenz auf bestimmte auBersprachliche Situationen zu beschreiben, fur seine lexikalische Bedeutung als notwen- 
dig angesehen werden, werden von WEINREICH als wesensimmanent (criterial) für das entsprechende Stichwort bezeichnet:

"Eine Denotationsbedingung kann als wesensimanent angesehen werden, wenn Informanten - vorausgesetzt, sie ist nicht erfiillt - sich weigern würden, den betreffenden Ausdruck zu verwenden ... Bedeutungen in naturlichen Sprachen enthalten oft Denotationsbedingungen, die nicht... definitiv wesensimmanent sind." (WEINREICH 1967, 33)

Die Frage, ob bestimmte Seme für die Bedeutung eines zu definierenden Lexems wesensimmanent oder - in gängigerer Terminologie - paradigmatisch sind, beschäftigt auch die Konstrukteure des MST. Sie erörtern dieses Problem beispielsweise anhand der semantischen Beschreibung des Verbs peč . Die Konstrukteure des MST verweisen auf gängige Definitionswörterbücher des Russischen, die dem Verb die unvollständige lexikographische Definition (7) zuweisen:

\section{(7) $\underline{p e c ̌ s}=$ 'Prigotovijat' $v$ pišču euchim nagrevaniem na żaru'}

Die Definition (7) erfaBt weder die faktitive Bedeutung von pe $\check{c}^{\prime}$., wie sie in den Wortverbindungen pe $\check{c}^{\prime}$ chleb und pe $\check{c}^{\prime}$ pirogi realisiert wird, noch die Bedeutung der Zustandsver-

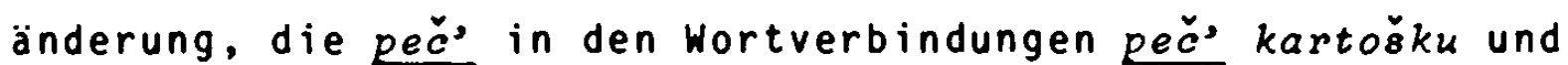
pečs jajca hat. Um die faktitive Bedeutung von pe čs vollständig zu explizieren, bedarf es daher eines Verweises darauf, daB die durch peč bezeichnete Handlung ihr objekt selbst hervorbringt ('dejstvie, sozdajušcee svoj ob-ekt'). Wird pečs in der Bedeutung einer Zustandsveränderung verwendet, ist seine lexikographische Definition nur vollständig, wenn sie einen Verweis darauf enthält, daB die Handlung auf einen bereits existierenden Gegenstand einwirkt ('vozdejatvie na uže suščestvujuščij, gotovyj predmet') (APRESJAN 1969a, 16). Die beiden oben genannten Bedeutungskomponenten sind daher für die faktitive bzw. die zustandsverändernde Bedeutung von pěc, paradigmatisch.

Da die Konstrukteure des MST sich bei der Bedeutungsexplikation ausschlieblich auf ihre Intuition berufen (vgl. S. 85 ff.), 
ist die Wesensimmanenz einiger Bedeutungskomponenten, die sie in den Bedeutungsexplikationen bestimmter Stichwörter ansetzen, sozusagen per definitionem nicht unumstritten. Neben der fehlenden Auffindungsprozedur für Seme, die für die Bedeutung eines Stichworts paradigmatisch sind, wird ihre Ermittlung noch dadurch erschwert, daB nicht alle Komponenten, die man ansetzt, um die Bedeutung des Lexems zu beschreiben, in konkreten Referenzakten auch immer aktualisiert werden mussen. Die Urteile verschiedener Analysatoren, über die Wesensimmanenz gewisser Seme für die Bedeutungen bestimmter Lexeme können sich daher unterscheiden. So erscheint es uns zweifelhaft, ob sämtliche Bedeutungskomponenten, die die Konstrukteure des MST in die lexikographischen Definitionen (8) - (11) von prosat', kurit', nakazyvat' und arestovyvat' einbringen, für die Bedeutungen der Stichwörter als paradigmatisch anzusehen sind:

(8) X prošaet $Y-a$ za $2=$ 'Imeja pravo nakazat' y-a za z neprijatnoe ili ne sčitaja vinu 2 dostatočno bol'šj, X otkazyvaetsja ispol'zovat' èto pravo.' (APRESJAN 1974b, 107)

(9) X kurit $Y=$ ' $Y$ vdychaet čerez rot neposredstvenno iz special'nogo ustrojstva $Y$ dym tlejušcego $v y-e$ veřsóstva 2 , obyčno - tabaka, $i$ vydychaet ètot dym - scel'ju, čtoby èto kauzirovalo $x$-a ispytyvat' prijatnoe oščščenie.' (AL'PERIN/APRESJAN/MEL'CUK $1975,18)$

(10) A nakazyvaet $B-a$ za C D-om = 'A pričinjaet $B-u$, soveršiv̌semu prostupok $C$, zlo $D$ s cel'ju ispravit' B-a.' (MEL'CUK 1974b, 134)

(11) X arestovyvaet $Y-a z a Z=$ 'Oficial'nye organy $X v$ sootvetstvii s predvaritel'nym rasporjaženiem lišajut $y-a$ svobody za ego dejstvija 2.' (MEL'CUK 1974b, 116) 
Die Wesensimmanenz, die die Konstrukteure des MST den in (8) - (11) unterstrichenen Semkomplexen für die Bedeutungen der Lexeme zuschreiben, scheint uns nicht ohne weiteres klar zu sein. Die durch prosat' bezeichnete auBersprachliche Situation setzt nicht voraus, daB der Mitspieler in der Agensrolle (' $X$ ') aufgrund seiner Position relativ zu dem Mitspieler in der Objektrolle ('Y') das Recht hat, letzteren zu bestrafen ('pravo nakazat' '). Ebenso ist es nicht zwingend, daB er das Motiv ('vina $Z Y-a$ ') seines Handelns unbedingt als nicht hinreichend gerechtfertigt ('ne sčitaja ... dostatočno bol'soj') ansehen muB.

Für die Verwendung von kurit' ist es fraglich, ob der Mitspieler in der Agensrolle (' $X$ ') die entsprechende Handlung immer ausführt, um eine gefühlsmäßige Befriedigung ('prijatnoe oščusčenie') zu erzielen. Gleichermaßen fraglich ist es, ob angesichts der Tatsache, daB eine Bestrafung oft ohne pädagogische ziele bzw. überhaupt ziellos erfolgen kann, in der Bedeutungsexplikation (10) von nakazyvat' der Zusatz 's cel'ju ispravit' $B-a$ ' in dieser Form unbedingt erforderlich ist. 26 Ebenfalls problematisch erscheint uns die Notwendigkeit, in die Bedeutungsexplikation (11) von arestovyvat' das Motiv der Handlung ('za ego dejstvija $Z^{\prime}$ ) in dieser Form anzugeben, da Verhaftungen oft grundlos vorgenommen werden (für weitere Beispiele vgl. S. 87 ff.). Für die lexikographischen Definitionen (8) - (11) bietet sich die Möglichkeit, die Obligatorik einzuschränken, mit der die Konstrukteure des MST die hier in Rede stehenden Semkomplexe als paradigmatisch für die Bedeutungen der entsprechenden Stichwörter ansetzen. Die Wesensimmanenz der betreffenden Bedeutungskomponenten kann durch Zusätze wie beispielsweise 'čašče vsego', 'obyčno', 'v bol'šinstve slučaev', 'v nekotorych slučajach' $u . \ddot{a}$. gegenüber den anderen Bedeutungskomponenten der lexikographischen Definition relativiert werden (vgl. S. 90). Für die Bedeutungsexplikation (11) von arestovyvat, ergibt sich in diesem Fall die Notwendigkeit, in der Satzform, in der die Konstrukteure des MST das Lexem definieren, den Ausdruck $z a z$ beispielsweise durch Einklammerung als fakultativ auszuweisen. 
Ein interessantes Beispiel einer lexikographischen Definition, die Bedeutungskomponenten enthält, welche die Konstrukteure des MST als paradigmatisch für das zu explizierende Lexem betrachten, obwohl sie redundant sind, fïhrt SCHMIOT an; vgl. die Bedeutungsexplikation (12) von peremešcat'sja:

(12) X peremeščaetsja iz $y-a v z=\cdot V$ moment $T_{1} X$ nachodits;a $v y-e, i v$ moment $T_{2} X$ nachoditsja $v$ Z-e, $i$ y ne sovpadaet $c$ 2-om, $i T_{1}$ re sovpadaet $8 T_{2}{ }^{-o m}$

(APRESJAN 1974b, 77)

In (12) wird mit $\mathrm{Hilfe}$ der Indices ' 1 ' und ' 2 ' die Information kodiert, daB der durch die Zeitvariable ' $T_{1}$ ' bezeichnete Zeitpunkt dem durch die Zeitvariable ' $T_{2}$ ' bezeichnetem Zeitpunkt vorausgeht. Der in (12) unterstrichene Semkomplex ist daher redundant (SCHMIOT 1981).

Im Zusammenhang mit der Stichhaltigkeit der Einwände, die wir gegen die Vollständigkeit und die Adäquatheit der oben genannten Bedeutungsexplikationen des EKW erhoben haben, mus allerdings, sozusagen einschränkend, auf ein schwieriges methodisch-methodologisches Problem hingewiesen werden. Es stellt sich zwangsläufig die Frage, ob letztlich uberhaupt in intersubjektiv entscheidbarer Weise uber die Guter von Btdeutungsexplikationen diskutiert werden kann, da die letzte Instanz, die hieruber entscheidet, immer die Intuition dessen ist, der diese Entscheidung fällt. Im Grunde ist es daher unmöglich, an der Güte einer semantischen Beschreibung unter Hinweis auf die Intuition eines anderen "objektive" Kritik zu uben, sofern nicht das gesamte Prinzip in Frage gestellt und durch ein geeigneteres Verfahren, wie beispielsweise in FAUST 1978 vorgeschlagen, abgelöst wird. Bedingt durch die Zielsetzung unserer Arbeit halten wir jedoch am Prinzip der Introspektion fest. Wir sind uns allerdings der Tatsache bewuBt, daB unsere Kritik an den oben genannten lexikographischen Definitionen unter Berücksichti. gung all der Vorbehalte betrachtet werden muB, die sich aus der hier besprochenen Problematik ergeben. 
Zu 3):

Die Forderung nach Tautologiefreiheit der lexikographischen Definitionen des EKW begründen die Konstrukteure des MST durch zahlreiche Verweise auf Bedeutungsexplikationen in gängigen Definitionswörterbuchern des Russischen, die sie als zirkulär betrachten. Sie werfen beispielsweise dem Wörterbuch von OZEGOV vor, die Lexeme pomošc', podderžka und sodejstvie sowie bol'šj und značitel'nyj zirkulär zu definieren, da in den Explikationen das Definiendum gleichzeitig zu seinem Definiens werde; vgl. die lexikographischen Definitionen (13) - (14):

(13) poddeř̌ka = 'Pomošc̆', sodejstvie'

(13') pomošc'

= 'Sodejstvie komu-n. v čëm-n.'

(13") sodejstvie

= 'Pomošč', podderžka v kakoj-n. dejatel'nosti'

(14) bol'šoj

' 'Inačitel'nyj po razmeram, po veličine, sile'

(14') značitel'nyj = 'Bol'šj po razmeram, sile' (APRESJAN 1966, 260; 1967, 10; 1969b, 4; vgl. S. 168 f. für weitere Beispiele tautologischer Definitionen)

Lexikographische Definitionen des oben genannten Typs explizieren die Bedeutungen der Stichwörter mit Hilfe sogenannter synonymischer Reihen (rjadovye opredelenija). Nach der Ansicht der Konstrukteure des MST führt die Explikation von Lexemen durch Synonyme dazu, daß zwischen Definiens und Definiendum keine klare Trennung vollzogen wird; auch widerspreche sie der unter 1) genannten Bedingung, in der die Konstrukteure des MST fordern, daß in lexikographischen Definitionen die Wortbedeutung in einfachere semantische Komponenten zerlegt werden müsse:

"Bei Explikationen des Types nesčst'e = 'Beda, gore', beda

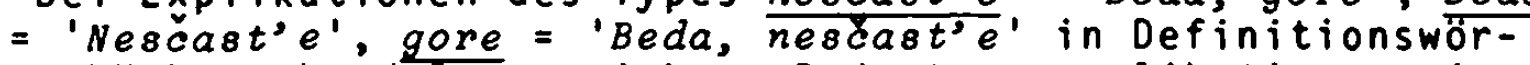
terbichern handelt es sich um Bedeutungsexplikationen mit Hilfe synonymischer Reihen ... Die Bedeutung des Ausgangsworts wird in ihnen durch ein Synonym, d.h. durch eine Bedeutung mit gleicher Komplexität definiert und nicht auf einfachere semantische Konstituenten reduziert. Die Explikation durch ein Synonym ist selbstverständlich völlig zuläsig, wenn exakte Bedeutungsgleichheit der Wortreihen 
vorliegt, aber dann muB wenigstens eins der Synonyme in semantische Konstituenten zerlegt werden. Geschieht dies nicht, so entsteht, wie die oben genannten Beispiele zeigen, in der Bedeutungsexplikation ein tautologischer Zirkel, der die Bedeutung undefiniert 1äBt." (APRESJAN 1969a, 13)

Die Konstrukteure des MST stellen keinerlei Erwägungen darüber an, ob die Effektivität des EKW als eines Definitionswörterbuches, das sich an russische Muttersprachler wenden soll, im Vergleich zu anderen russischen Definitionslexika durch nichtzirkuläre Definitionen erhöht werden kann. In ihren Arbeiten findet sich lediglich eine einzige Stellungnahme von MEL'CUK, in der dieser tautologischen lexikographischen Definitionen keine negative Folgen für die lexikographische Praxis beizumessen scheint:

"Für praktische Ziele, d.h. wenn man sich an den Menschen wie beispielsweise in Definitionswörterbijchern wendet, werden Bedeutungen oft durch Verweise auf Synonyme oder Quasisynonyme beschrieben: Im 'Slovar' russkogo jazyka' von ožegov (19533) wird položit'sja durch doverit'sja und ponadejat'sja, doverit'sja durch projavit' doverie und polozit'8ja erlautert. Für wissenschaftliche Zwecke ist ein derartiges Vorgehen absolut ungeeignet!" (MEL'CUK 1970, 205)

In der Tat muB gefragt werden, welchen Beitrag tautologiefreie Bedeutungsexplikationen eigentlich in nichtfachsprachlichen Definitionswörterbüchern leisten, um die Güte der Definitionen im Vergleich zu zirkulären Explikationen zu erhöhen. Das Tautologieverständnis, von dem die Konstrukteure des MST sowohl bei der Kritik der Definitionspraxis gängiger russischer Wörterbucher als auch bei der Konstruktion der lexikographischen Definitionen des EKW ausgehen, vernachlässigt völlig den wesentlichsten Aspekt der lexikographischen Praxis - den potentiellen Wörterbuchbenutzer. Dies ist um so bedauerlicher, als sie gerade ihm, d.h. dem russischen Muttersprachler, ein Wörterbuch zur Verfügung stellen wollen, das u.a. im Bereich der Wortdefinitionen gängigen Definitionswörterbüchern des Russischen überlegen sein soll.

Wir schlieBen uns daher der Auffassung von ARBATSKIJ an, der den Konstrukteuren des MST vorwirft, in ihrer Kritik an lexikographischen Definitionen gängiger russischer Defi- 
nitionswörterbücher ein falsches Tautologieverständnis zugrundezulegen. ARBATSKIJ bezeichnet den Tautologiebegriff, den sich die Konstrukteure des MST zueigen machen, als einen formalen, da er ausschlieBlich auf die Ausdrucksseite der Definitionen bezogen sei:

"... die Tautologie wird aus der Sicht der Form, der Struktur der Definition betrachtet. Sie wird in diesem Fall als eine Gemeinsamkeit von Definiendum und Definiens der Definition angesehen. Hinsichtlich des Wesens der Tautologie hegen Apresjan und einige andere Autoren keinerlei Zweifel. Ohne zu zögern legen sie sich auf eine rein formale Behandlung der Tautologie als Verwendung der Sprache des Definiendum in der Sprache des Definiens fest und machen sie zur Grundlage der Analyse von Definitionswörterbiuchern ... diese Auffassung deckt das Wesen der Tautologie nicht auf, sie verfälscht es eher." (ARBATSKIJ 1974, 56-57)

ARBATSKIJ fordert, für die kritik und konstruktion von lexikographischen Definitionen in erklärenden Wörterbüchern den formalen Tautologiebegriff zugunsten eines angemesseneren inhaltlichen Tautologieverständnisses aufzugeben. Wird die Tautologie als inhaltiche Erscheinung der Definition aufgefaBt, so betrachtet man sie als:

"... Unzulänglichkeit der Information über den zu definierenden Gegenstand oder als Wiederholung desselben Begriffs oder Gedankens im Definiens der Definition, der bereits in inrem Definiendum fixiert ist." (ARBATSKIJ 1974, 56)

Ausgehend von einem inhaltlichen Tautologieverständnis kritisiert ARBATSKIJ die Haltung der Konstrukteure des MST, u.a. lexikographische Definitionen vermittels sogenannter synonymischer Reihen (s.o.) als zirkulär anzusehen. In seinen oberlegungen berücksichtigt ARBATSKIJ - im Gegensatz zu den Konstrukteuren des MST - die potentiellen Adressaten eines nichtfachsprachlichen einsprachigen Definitionswörterbuchs. ARBATSKIJ bestreitet, daB Sprecher die Bedeutungen der durchweg usuellen Lexeme, die im EKW und anderen nichtfachsprachlichen russischen Definitionswörterbüchern erklärt werden, nicht kennten. ARBATSKIJ bestreitet die Zirkularität bestimmter lexikographischer Definitionen des Wörterbuchs von OZEGOV, die APRESJAN wie beispielsweise nesčast'e = 'Beda, gore', beda = 'Nesčast' $e^{\prime}$ und gore $=$ 'Beda, nesčast' $e^{\prime}$ 
als tautologisch betrachtet, da:

"... die erklärenden Wörter in jeder der drei Definitionen dem Leser des Wörterbuchs hinreichend verständlich sind und alle erforderliche Information uber die Bedeutung der $z u$ erklärenden Wörter enthalten." (ARBATSKIJ 1974, 58)

Wir halten ARBATSKIJs Forderung, bei der Analyse von lexikographischen Definitionen ein inhaltliches Tautologieverständnis zugrundezulegen, für richtig, auch wenn er seine Oberlegungen nicht dahingehend weiterfüht, daß er die prinzipielle Notwendigkeit von nichtfachsprachlichen einsprachigen Definitionswörterbüchern für die lexikographische Praxis in Frage stellt, wie es WEINREICH in seiner entsprechenden kurios iberhöhten Stellungnahme tut (vgl.S.65). ARBATSKIJs Tautologieverständnis erscheint uns für die Bewertung und Konstruktion lexikographischer Definitionen angemessener als das Tautologiekonzept der Konstrukteure des MST zu sein, da es den potentiellen Adressaten des Wörterbuchs berücksichtigt. Die Entscheidung, ob eine gegebene Bedeutungsexplikation zirkulär oder nichtzirkulär ist, kann immer nur im Hinblick auf den postulierten bzw. konkreten Wissensstand der Gruppe von Sprechern gefällt werden, an die sich das Wörterbuch wendet. Die Tatsache, daB weder die Konstrukteure des MST noch die Autoren anderer gängiger Definitionswörterbucher die potentiellen Adressaten ihrer Nachschlagewerke spezifizieren, ist kein Grund, bei der Beurteilung der Zirkularität von lexikographischen Definitionen das inhaltliche Tautologiekonzept zu verwerfen:

"... bei der Ermittlung der Zirkularität (tavtologičnost") oder der Nichtzirkularität (netavtologičnost') der einen oder anderen Definition kommt der Festlegung des Wissensstands der Leser, für die sie bestimmt ist, eine entscheidende Bedeutung $z u$. Bei der Bedeutungsexplikation gehen moderne Definitionswörterbücher zweifellos immer von einem bestimmten Entwicklungsstand des Lesers aus, obwohl sie ihn nicht klar und deutlich formulieren. Zweifellos stützen sie sich vornehmlich auf den Kenntnisstand von Nichtspezialisten. Dieser Entwicklungsstand bestimmt den konkreten Inhalt aller lexikographischen Definitionen dieser Wörterbiucher. In den theoretischen Oberlegungen APRESJANs wird irgendein konkreter Entwicklungs-oder Wissensstand der Leser nicht nur nicht vorausgesetzt, sondern sogar völlig abgelehnt." (ARBATSKIJ 1974,61-62) 
Wir glauben, daB das EKW, das sich an den russischen Muttersprachler wendet, Wortbedeutungen ohne weiteres durch Explikationen des Typs bol'šj $\leftrightarrow$ značitel'nyj, mebel' $\leftrightarrow$ obstanovka, utračivat' $\leftrightarrow$ lišat'sja usw. definieren kann, vorausgesetzt, es handelt sich um genaue Synonyme. Es ist wenig realistisch, den potentiellen Adressaten des Wörterbuchs ein Bedurfnis zu unterstellen, die lexikalischen Bedeutungen usueller, nichtfachsprachlicher Lexeme nachschlagen zu wollen, da sie diese bereits kennen. Bedeutungsexplikationen des oben genannten Typs sind daher formal, jedoch nicht inhaltich tautologisch.

Zwar setzen wir durch unsere forderung das unter 1) genannte Postulat nach sukzessiver Dekomposition der zu explizierenden Bedeutungen in einfachere Komponenten auBer Kraft, hierzu sind wir aber berechtigt, denn aus der sicht der potentiellen Wörterbuchbenutzer ist es wenig sinnvoll, diese Forderung aufrechtzuerhalten. Im übrigen ist die Anzahl genaver Synonyme in natürlichen Sprachen gering, so daß nur wenige lexikographische Definitionen von unserem Verbesserungsvorschlag betroffen werden ( $v g l$. für Beispiele genauer lexikalischer Synonyme im Russischen APRESJAN 1974b, $224 \mathrm{ff}$.). Die Bedeutungsexplikation mit Hilfe von genauen Synonymen sollte nur in solchen Fällen aufgegeben werden, wenn Lexeme $z u$ definieren sind, von denen angenommen werden $k a n n, d a \beta$ die potentiellen Wörterbuchbenutzer, die vorher festzulegen sind, sie nicht kennen. Tautologisch für Sprecher ohne fachsprachliche Spezialkenntnisse sind beispielsweise die von ARBATSKIJ genannten Explikationen kuguar $\leftrightarrow$ puma, kafe ?' $\leftrightarrow$ $\underline{i z r a z e c}$, solo $\leftrightarrow$ ispolnjaemyj solo und mikrob $\leftrightarrow$ bakterija $\leftrightarrow$ bacil2a (ARBATSKIJ 1974, 59,61).

Wenn wir für das EKW als ein nichtfachsprachliches einsprachiges Definitionswörterbuch, das praktische Bedürfnisse von Sprechern befriedigen soll, die Forderung der Konstrukteure des MST nach formaler Tautologiefreiheit seiner lexikographischen Definitionen nicht akzeptieren, so stellen wir deren Berechtigung für das EKW als wissenschaftliches Definitionswörterbuch keinesfalls in Frage. Betrachtet man das EKW als ein Definitionswörterbuch, das sich an den Linguisten 
wendet und Wortbedeutungen nach theoretischen lexikologischen Prinzipien beschreibt, ist das Postulat nach formaler Tautologiefreiheit der Bedeutungsexplikationen selbstverständlich begründet:

"Eine theoretische (d.h. wissenschaftliche) Beschreibung irgendeines Gegenstandes zu geben, bedeutet vor allem einige elementare (zumindest einfachere als der Gegenstand selbst) Komponenten anzugeben, aus denen er besteht ..." (MEL'CuK 1970,205 )

2.2.1.3 Das Konzept der lexikalischen Bedeutung im erklärend-kombinatorischen Wörterbuch

In Anlehnung an verschiedene linguistische und sprachphilosophische Bedeutungskonzepte (Z̈OLKOVSKIJ/MEL'CUK 1969, 13; MEL'CUK 1974b, 10; APRESJAN 1963, 106; 1974b, 56 ff.; HELBIG 1974, 108) verstehen die Konstrukteure des MST die Bedeutung sprachlicher Einheiten (smys 2 ; bzw. fiur Einzellexeme: leksičeskoe značenie) als deren Potenz, sich auf bestimmte Gegenstände oder Sachverhalte der auBersprachlichen Wirklichkeit zu beziehen. Die Bedeutung sprachlicher Zeichen ist daher eng mit deren Referenz verbunden; m.a.W., Sprecher können mit Hilfe der Bedeutung sprachlicher Zeichen auf die auBersprachliche Wirklichkeit referieren. Versteht man die Bedeutung sprachlicher Zeichen als deren Potenz oder Disposition zur Referenz, so interessiert man sich - analog zur langue/parole- bzw. Kompetenz/Performanz-Unterscheidung lediglich auf einer relativ abstrakten Betrachtungsebene für den konkreten Gebrauch, den Sprecher von dieser Eigenschaft sprachlicher Zeichen in konkreten Referenzakten machen. Man abstrahiert von der Gesamtheit der in der parole möglichen Verwendungen einer sprachlichen Einheit, indem man aus deren Menge bestimmte rekurrente, möglichst invariante Vorkommen ausgrenzt, zu Referenztypen oder -klassen zusammenfaßt und je nach dem Grad ihrer semantischen Varianz untereinander die entsprechende Einheit als monosem, polysem oder homonym klassifiziert. In einer linguistischen 
Semantik, die die Bedeutung sprachlicher Zeichen als deren Referenzpotential auffaBt, braucht daher die Aktualisierung dieses Potentials in Kommunikationsakten, kurz auch Meinung genannt, nicht unbedingt erschöpfend beschrieben zu werden.

Die Konstrukteure des MST betrachten die Bedeutung sprachlicher Zeichen als komplexe, uberindividuelle Begriffe (signifikativnoe, ponjatijnoe, myslitel'noe značenie), die Sprecher durch Abstraktionen ihres Erkennens der auBersprachlichen Wirklichkeit gewonnen haben. Ihr Bedeutungskonzept kommt daher FREGEs Konzept des Sinns sehr nahe. Nach KUTSCHERA versteht FREGE unter dem Sinn

"... eine begriffliche, platonistische Entität, d.h. etwas Intersubjektives, Objektives und streng von den subjektiven Vorstellungen und Assoziationen zu Unterscheidendes ..." (KUTSCHERA 1971,145 )

Eine weitere charakteristische Eigenschaft des Bedeutungskonzepts der Konstrukteure des MST besteht darin, daB sie Bedeutungen von Einzellexemen auf der syntagmatischen Achse in Form reduzierter Aussagen - ZOLKOVSKIJ spricht in diesem Zusammenhang von "soobščenija" (ZOLKOVSKIJ 1964, 10) - beschreiben, die sich von konkreten Sätzen nur dadurch unterscheiden, daß die Leerstellen fur die Mitspieler, die an der durch das Stichwort typischerweise bezeichneten Situation beteiligt sind, durch Gegenstandsvariable und nicht durch konkrete lexikalische Auffüllungen eingenommen werden (vgl. S.65, $190 \mathrm{ff}$. SOwie APRESJAN 1968, 41; LORENZ/WOJTAK 1977, 128). Dieses Verständis der lexikalischen Bedeutungen hat zur Folge, daB die semantischen Beschreibungen, die die Konstrukteure des MST für Wortschatzeinheiten im EKW geben, den Rahmen der für Wörterbücher üblichen Wortsemantik sprengen und satzsemantischen Deskriptionen sehr nahe kommen. In der Abwendung von der Wortsemantik erblickt PADUCEVA ubrigens eines der typischen Merkmale der modernen linguistischen Semantik:

"Eine der wichtigsten Verschiebungen in der modernen Semantik im Vergleich zur traditionellen besteht darin, daB die Tatsache klarer erkannt wird, daB zur Aufgabe der Semantik nicht nur die Beschreibung der Bedeutungen von Einzelwör- 
tern, sondern auch die ganzer Sätze gehört." (PADUCEVA 1974, 11-12)

Unter den Begriff der lexikalischen Bedeutung subsumieren die Konstrukteure des MST nicht nur die signifikative Bedeutung eines sprachlichen Zeichens, die es ermöglicht, Referenz herzustellen, sondern auch dessen strukturelle Bedeutung, d.h. die Fähigkeit, paradigmatische semantische Beziehungen zu anderen sprachlichen Zeichen einzugehen:

"Die strukturelle Bedeutung ist die Beziehung von Zeichen zu anderen Zeichen ...; die Beziehung von Zeichen zu anderen Zeichen auf der paradigmatischen Achse kann als differentiale Bedeutung oder in voller Obereinstimmung mit der Idee SAUSSUREs als Wert (znacimost") bezeichnet werden." (APRESJAN 1963,106 )

Von der lexikographischen Beschreibung des russischen Wortschatzes im EKW fordern sie daher u.a., daB sie

"... die paradigmatischen Eigenschaften im Bereich der lexikal ischen Bedeutungen oder die distinktiven semantischen Merkmale eines Wortes..." (APRESJAN 1968, 34)

erfassen solle. Das Ziel einer semantischen Beschreibung, die die Bedeutungen sprachlicher Zeichen ähnlich dem SAUSSUREschen Verständnis ihres Wertes (valeur) ausschlieblich als ihre strukturelle Bedeutung betrachtet, wird häufig auch als die Ermittlung der "Struktur des Lexikons" bezeichnet. Wir haben bereits auf die Schwierigkeiten hingewiesen, die das Postulat nach Vollständigkeit der semantischen Beschreibung, das die Konstrukteure des MST an die lexikographischen Definitionen des EKW richten, insbesondere für die Erfassung der signifikativen Bedeutung mit sich bringt (vgl. S. $71 \mathrm{ff}$.). Die Problematik, die das Vollständigkeitspostulat für die Beschreibung der begrifflichen Bedeutung sprachlicher Zeichen aufwirft, veranlaBt WEINREICH zu der Forderung, die Modellierung der strukturellen Bedeutung in den Mittelpunkt lexikographischer Bemühungen zu stellen:

"Die Konsistenz der Lexikographie könnte verbessert werden, wenn die Wörterbuchautoren an der Annahme festhielten, daB die Ausdrïcke einer Sprache im groBen und ganzen komplementär sind. Aus dieser Annahme folgt, daB der wichtigste Fall, 
mit dem sich die semantische Beschreibung zu beschäftigen hat, darin besteht, daB die Bedeutung eines Ausdrucks dort endet, wo die andere beginnt. Eine semantische Beschreibung sollte im allgemeinen nicht 'absolute' Definitionen anstreben, sondern solche, die die Bedeutung eines Ausdrucks von denen anderer Ausdrücke mit ähnlichen Bedeutungen (Synonyme) abgrenzen." (WEINREICH 1967, 30)

WEINREICHs Vorschlag, sich in der Lexikographie und der linguistischen Semantik in erster Linie auf strukturelle Beschreibungen zu konzentrieren, indem man Bedeutungsgleichheiten und - unterschiede zwischen Wortschatzeinheiten herausarbeitet, ist im Vergleich zu einer semantischen Beschreibung, die die signifikative Bedeutung vollständig erfassen will, zweifellos einfacher zu verwirklichen. Wüde man sich jedoch in Lexikographie und linguistischer Semantik ausschlieBlich auf die Modellierung der Struktur des Lexikons beschränken, so blieben zwei wesentliche Aspekte sprachlicher Zeichen unberücksichtigt:

a) Die Fähigkeit von Sprechern, mit Hilfe der Bedeutung sprachlicher Zeichen Referenz auf die auBersprachliche Wirklichkeit herzustellen (s.0.), würde nicht beschrieben.

b) Die Fähigkeit von Sprechern, kraft ihres Wissens um die Bedeutungen sprachlicher Zeichen SchluBfolgerungen zwischen den Bedeutungen von Sätzen $z u$ ziehen, in denen sie vorkommen, d.h. aufgrund sinnrelationaler Eigenschaften von Sätzen synonymische Paraphrasierungen im Sinne der Konstrukteure des MST vorzunehmen (vgl. S. $3 \mathrm{ff.}$ ), würde nicht erfaBt.

Aus diesem Grund bemühen sich die Konstrukteure des MST, im EKW sowohl die strukturelle als auch die signifikative Bedeutung von Lexemen zu beschreiben.

Um Lexembedeutungen zu beschreiben, bedienen sich die Konstrukteure des MST, wie bereits kurz angesprochen (vgl.S. 73) des Verfahrens der Introspektion:

"Auf die Frage, wie man zur Auswahl der einen oder anderen Beschreibung eines konkreten signifié gelangt, müssen wir antworten, daB uns keine formalen Prozeduren zur Verfugung 
stehen, die uns zur Aufdeckung der inneren Strukturen des betreffenden signifiés führen könnten; es ist jedesmal eine Angelegenheit der Kompetenz und Intuition des Forschers; d.h., strenggenommen erfolgt die Beschreibung der signifiés arbiträr." (MEL'CUK 1974b, 78)

In seiner Replik auf eine Arbeit von SVEDOVA (SVEDOVA 1970) unterstreicht APRESJAN mit Nachdruck die Vorteile, die das introspektive Vorgehen des Linguisten (sub-ektivnyj opyt issiedovatelja) bei der semantischen Beschreibung gegenüber Untersuchungen begrenzter Korpora sprachlicher Daten auszeichnet:

"Obwohl das subjektive Experiment weniger repräsentativ als Wörterbücher und Wortkarteien ist, verfügt es gegenüber letzteren über den unbestreitbaren Vorteil, nicht nur die Möglichkeit des Beobachtens, sondern auch des Experimentierens zu eröffnen - eine Möglichkeit, die solche hervorragenden Sachkenner wie PESKOVSKIJ und SCERBA hoch bewertet haben. Im Experiment findet sich reichlich 'negatives sprachliches Material' (SCERBA), das in der Hinsicht einzigartig ist, als es um ein vielfaches schneller und effektiver als Texte erlaubt, wesentliche Elemente der Wortbedeutung zu ermitteln." (APRESJAN 197la, 34)

Das introspektive Vorgehen der Konstrukteure des MST bei der Bedeutungsbeschreibung wollen wir in Anlehnung an SCHWARZE als Homogenitätsannahme bezeichnen (SCHWARZE 1975, 73, 144). Hierunter verstehen wir eine kontrollierte Reduktion oder Idealisierung, der der Linguist bestimmte Aspekte seines Forschungsgegenstands unterzieht. Die Homogenitätsannahme, die die Konstrukteure des MST im Hinblick auf die Beschreibung von Lexembedeutungen im EKW machen, besteht darin, daB sie die individuelle Variation, der Wortbedeutungen innerhalb einer Sprachgemeinschaft von Sprecher zu Sprecher unterliegen können, ausblenden und ihr intuitives Wissen um die Bedeutungen der Lexeme - ihre Kompetenzbedeutungen in der Terminologie von LORENZ/WOTJAK 1977, 98 - als repräsentativ für das Wissen sämtlicher Mitglieder der russischen Sprachgemeinschaft ansehen. Die Homogenitätsannahme der Konstrukteure des MST führt unweigerlich dazu, daB die lexikographischen Definitionen, die sie im EKW den Lexemen zuweisen, nicht unbedingt auch der Intuition seiner potentiellen Adressaten entsprechen muissen. Die Konstrukteure des MST nehmen 
daher - wie auch andere Lexikographen - zwangsläufig die Folgen in Kauf, die sich aus dem Umstand ergeben, daB die lexikographischen Definitionen im EKW im Sinne WEINREICHs "Ein-Mann-Hypothesen" bzw. "Kollektivhypothesen" (WEINREICH 1967, 42) über die Bedeutungen der zu explizierenden Lexeme sind. Die Konsequenzen introspektiven Vorgehens in der 1 inguistischen Semantik machen sich immer dann deutlich bemerkbar, wenn verschiedene Analysatoren zu unterschiedlichen Entscheidungen über die Angemessenheit bestimmter Teile der Bedeutungsexplikation eines Lexems gelangen, so daB eine Diskussion um die Gute oder Adäquatheit der lexikographischen Definition entsteht (vgl. S. $74 \mathrm{ff.}$ ).

Gemäß dem Urteil eines russischen Muttersprachlers lassen sich beispielsweise die Explikationen (15) und (16), die die Konstrukteure des MST für die Verben zavidovat' und echat' geben, nicht aufrechterhalten.

Unser Informant bestritt, daB die unterstrichenen semantischen Komponenten für die Bedeutungen dieser Verben paradigmatisch seien:

$$
\begin{aligned}
& X \text { zaviduet } Z-u Y-a / \text { ' } X \text { ne imeet } Z-a \text {, } i \text { imeet } 2 \text {, } \\
& y-u v z-e / Y-u \text { iz-za i X ispytyvaet otricatel'nuju } \\
& z-a=\quad \text { èmociju, kauziruemuju želaniem, }
\end{aligned}
$$

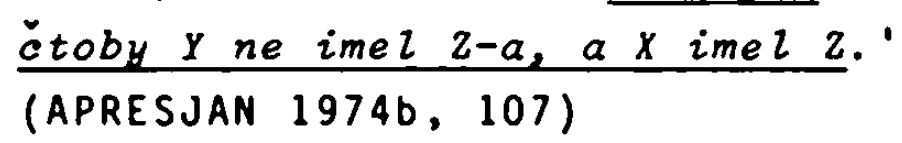

(16) A edet iz $Y-a v 2$ na $W-e=$ 'A peremě̌čaetsja iz $Y-a v$

2, potomu čto A nachoditsja

na W-e, kotoroe peremeřčaetsja

iz $Y-a v 2$, i pereměsčenie

iz $Y-a v z$ vchodit $v$ čis 20

celeja-a' (APRESJAN $1974 \mathrm{~b}$, 208)

Aus den lexikographischen Definition (15) und (16) wird deutlich, daB die Konstrukteure des MST bestimmte Ausschnitte ihres Wissens uber die Referenten (Situationen), auf die man sich mit Hilfe der Verben zavidovat' und echat' beziehen kann, als semantische Komponenten in die Bedeutungsbeschreibungen der Lexeme aufgenommen haben. Diese Semkomplexe werden zwar 
in bestimmten Verwendungen aktualisiert, sie entsprechen jedoch nicht unbedingt all denjenigen Verwendungen der Wörter, die man als fur diese typisch oder usuell ansehen kann. Die Bedeutungsexplikation (15), die die Konstrukteure des MST für zavidovat' geben, erfaBt am ehesten ausschlieblich solche Verwendungsweisen des Verbs, in denen das Motiv des Neids durch Gegenstände hervorgerufen wird, die in einer gegebenen Welt nur in einem einzigen Exemplar existieren. Eine solche Verwendung von zavidovat, liegt in Satz (17) vor:

(17) Moskviči zavidujut Leningradcam iz-za Admiral'testva, a Leningradoy zavidujut Moskvičam iz-za Krasnoj ploščadi.

Im übrigen weist die lexikographische Definition (15) von zavidovat' erhebliche Mängel auf. Sie trägt der Tatsache nicht Rechnung, daß man jemanden nicht nur um Gegenstände, sondern auch um psychische Eigenschaften und Fähigkeiten beneiden kann. Der Semkomplex '... želaniem, čtoby y ne imel 2-a, a $X$ ime $Z$ 2.' wird in dieser Verwendung des Verbs nie realisiert; vgl. Satz (18):

(18) P̈̈tr zaviduet duševnomu ravnovesiju ženy.

Um diese Unzulänglichkeiten zu beheben, bedarf es, falls man das Verb nicht in zwei polyseme lexikalische Einheiten aufspalten will, einer Korrektur der Bedeutungsexplikation (15). Im Zusammenhang mit der lexikographischen Definition (15) stellt sich weiterhin die Frage, inwieweit in ihr zu berücksichtigen ist, ob es sich bei dem Motiv des Neids, sofern dieser durch einen Gegenstand hervorgerufen wird, um ein ständiges oder nur vorübergehendes Besitzgut des Mitspielers in Objektrolle handelt.

Eine Bedeutungsexplikation von zavidovat', die die oben genannten Verwendungsweisen des Verbs weitgehend erfaBt und daher der lexikographischen Definiton (15) vorzuziehen ist, gibt (bereits 1970!) IORDANSKAJA; vgl. die Bedeutungsexplikation (19):

(19) A zaviduet $C-u v B-e=$ 'A dosaduet na otsutstvie u $A-a$ fakta $B$, kotorij $A$ želaet imet', i nalióie fakta $B$ u $c-a$. 
Die lexikographische Definition (16), die die Konstrukteure des MST für echat' geben, erfaBt einen Verwendungstyp des Verbs, durch den eine intentional kausierte und intentional zielgerichtete Bewegung bezeichnet wird. Bei einer Verwendungsweise von echat, wie beispielsweise in Satz (20) wird der Semkomplex '... i peremeščenie iz $Y-a v z$ vchodit $v$ čislo celej $A-a . '$ jedoch nie aktualisiert:

(20) Zabyv sdelat' peresadku, Larisa echala na èlektričke iz Sestroreska v Vyborg.

In den Adäquatheitsurteilen, die unser Informant zu den beiden Bedeutungsexplikationen (15) und (16) des EKW abgab, wird die bereits angesprochene Problematik der Homogenitätsannahme deutlich. Sie zeigt sich darin, daB es im Hinblick auf bestimmte Bedeutungskomponenten, die verschiedene Analysatoren fur die Bedeutungen der beiden oben genannten Lexeme geltend machen, nicht unumstritten ist, ob sie für diese Wörter paradigmatisch sind. Es liegt daher nahe, den semantischen Komponenten, die in einer Bedeutungsbeschreibung angesetzt werden, keinesfalls Anweisungscharakter zuzusprechen, indem man sie als Denotationsbedingungen auffaBt, die allesamt erfïlt sein müssen, um mit Hilfe der Wörter Referenz herstellen zu können. Bedeutungen werden nicht in der Weise realisiert, daB die Auslassung einer oder mehrerer ihrer Komponenten die Denotation nicht mehr gewährleistet.

Die negativen Folgen, die die Homogenitätsannahme in der linguistischen Semantik in bestimmten Fällen für die Güte semantischer Beschreibungen nach sich ziehen kann, sollte nicht iberbewertet werden oder gar angesichts der Probleme, die empirische Untersuchungen durch Informantenbefragungen aufwerfen (s.u.), dazu veranlassen, die semantische forschung in der Linguistik völlig einzustellen. Da sich die Bedeutungen sprachlicher Zeichen der direkten Beobachtung entziehen und of $t$ uber ein hohes MaB an Unbestimmtheit oder Unschärfe verfügen, besteht das Verfahren, das für den Linguisten noch am ehesten realisierbar ist, darin, introspektiv vorzugehen. 27 selbstverständich sollte er immer versuchen, die intuitiv gewonnenen Beschreibungsergebnisse, 
falls möglich, durch empirische Untersuchungen abzusichern. Wegen des großen Zeit- und Materialaufwands durfte dies jedoch beim heutigen Stand linguistischer Forschung weitgehend der Idealfall bleiben.

Im Zusammenhang mit empirischen Untersuchungen im Bereich der Semantik, die AufschluB iber die Güte von Bedeutungsbeschreibungen geben sollen, stellt sich auBerdem noch ein methodologisch-erkenntnistheoretisches Problem. Es dürte schwierig sein, zu entscheiden, ob man bei entsprechenden Informantenbefragungen auch wirklich den Gebrauch testet, den die Versuchspersonen von einem Lexem in konkreten Kommunikationsakten machen oder ob nur deren Wissen um die Bedeutung der gegebenen Einheit erfaBt wird.

Es ist erstaunlich, wie wenig die Konstrukteure des MST in ihren Arbeiten über die lapidare feststellung hinaus, da $B$ sie bei der semantischen Beschreibung intuitiv vorgingen, über die Schwierigkeiten nachdenken, die die Introspektion mit sich bringt, obwohl sie in bestimmten Bedeutungsexplikationen das Problem der Wesensimmanenz bestimmter semantischer Komponenten für die zu definierende Wortbedeutung durchaus berücksichtigen. Ist die Wesensimmanenz bestimmter Bedeutungskomponenten unklar, spezifizieren die konstrukteure des MST sie durch Zusätze wie 'obyčno' oder 'naprimer'; vgl. die lexikographischen Definitionen (21) - (24) von kurit', streljat', čajnik und jasnyj in einer ihrer Bedeutungen:

(21) $X \underline{\text { kurit }} Y=$ ' $X$ vdychaet čerez rot neposredstvenno iz special'nogo ustrojstva $Y$ dym tlejušcego $v y-e$ veřcestva 2 , obyčno - tabaka, $i$ vydychaet ètot dym - s cel'ju, čtoby èto kauzirovazo $X-a$ ispytyvat' prijatnoe ošcuščnie.' (AL'PERIN/APRESJAN/MEL'CUK $1975,18)$

(22) X streljaet $Y$ u $2-a=$ 'X prosit $Z-a$, obyčno maloznakomogo $i l i$ neznakomogo $y-u$, čtoby $Z$ dal $x-u$ veršc $Y$, kotoraja imeet neznačitel'nuju cennost' 


\section{i nebol'soj razmer.' (APRESJAN/ \\ ZOLKOVSKIJ/MEL'CUK 1975, 41)}

(23) $x-\underline{c a j n i k}=' x-$ sosud, prednaznačennyj dija kipjačenija vody dlja čaja ...., ili dija zavarivanija ... čaja, ili dlja podači čaja ... na stol, - obyčno polaja (poly) sfera ili cilindr iz metalla ili farfora ... s ručkoj, kryškoj i nosikom ...'

(APRESJAN/Z̈OLKOVSKIJ/MEL'CUK 1975, 47)

(24) $x$ - jasnyj $=' x$ - charakterizujuščijsja otsutstviem $v$ atmosfere častic (naprimer)=oblaka, py $l^{\prime}$, tuman, dym ...), prepjatstvujuščich vosprijatiju sveta.' (KOSSEK 1973, 38)

Die Konstrukteure des MST präzisieren das Vollständigkeitspostulat, das sie sowohl an die lexikographischen Definitionen des EKW als auch an die semantische Repräsentation von Texten im MST richten, durch fünf dezidierte Annahmen über den Umfang der semantischen Beschreibung:

1) Die Bedeutung sprachlicher Zeichen soll nur in dem AusmaB beschrieben werden, daB das "naive" Weltbild von Sprechern erfaBt wird:

"Die Definition eines Stichworts sollte nicht uber die gängige Vorstellung von seiner Bedeutung hinausgehen, die ihrer usuellen Verwendung durch den Durchschnittssprecher (man in the street) zugrundeliegt. Sie sollte keine 'enzyklopädischen' Aspekte der Wortbedeutung berücksichtigen, die in ihrem terminologischen Gebrauch durch einen Fachmann aktualisiert werden; vgl. den Unterschied im Verständnis und Gebrauch der Wörter jagoda, prestuplenie, priby $z^{\circ}$ und anderer durch einen Durchschnittssprecher (sogar einen Intellektuellen!) bzw. durch einen Botaniker, Juristen und okonomen." (APRESJAN/ŽOLKOVSKIJ/MEL'CUK $1969 b, 4)$

"Will der Lexikograph nicht den Boden seiner Wissenschaft verlassen und zum Enzyklopäden werden, so besteht seine Aufgabe darin, das naive Weltbild in den lexikalischen Bedeutungen der Wörter aufzudecken und es im System der Explikationen abzubilden." (APRESJAN 1974b, 58)

"Die Explikation ... soll ausschließlich diejenige usuelle Vorstellung über die Bedeutung der Wörter modellieren, die dem Sprachgebrauch eines 'durchschnittlichen' Spre- 
chers zugrundeliegt. Sie kann und soll nicht den genaueren oder detaillierteren Inhalt berücksichtigen, der dem Wort bei seinem terminologischen Gebrauch durch Spezialisten verliehen wird (vgl. beispielsweise den Unterschied im Verständnis des Wortes agressija durch einen Durchschnjttsleser oder einen Völkerrecht ler oder des Wortes pozar durch einen Zuschauer oder einen Feuerwehrmann)." (MEL, CuK 1974b, 111)

"Inhaltlich modelliert sie [d.h. die lexikographische Definition des EKW; K.H.] die Vorstellung der Wortbedeutungen bei einem durchschnittlichen Sprecher und ist begründet auf eine Weltsicht oder einen Wissensstand, der einen solchen Sprecher entspricht." (REUTHER 1978, 26)

2) In der Bedeutungsbeschreibung sollen auch die sprachlichen Erscheinungen berücksichtigt werden, die unter die Pragmatik des Zeichens fallen, d.h. die im Sinne von MORRIS das Verhältnis zwischen dem Zeichen und seinen Benutzern betreffen (APRESJAN 1974b, $67 \mathrm{ff.}$ ). Die Konstrukteure des MST sehen im Wörterbuchartikel des EKW zwei verschiedene Bereiche - die konnotative Zone (konnotativnaja zona) und den modalen Rahmen (modal'naja ramka) vor, in denen sie gegebenenfalls pragmatische Informationen uber das zu explizierende Stichwort festhalten:

"Die Konnotationen muissen in einer speziellen pragmatischen oder konnotativen Zone des entsprechenden Wörterbuchartikels angegeben werden. Sie dienen als Grundlage fuir die Explikation solcher übertragener Bedeutungen eines Wortes, die mit seiner Grundbedeutung keine gemeinsamen semantischen Merkmale haben. Was diejenigen pragmatischen Elemente des Zeichens angeht, die als modaler Rahmen bezeichnet werden und in denen die Einschätzung der auBersprachlichen Situation durch den Sprecher oder den Hörer zum Ausdruck kommt, so müssen diese... direkt in die Bedeutungsexplikation des Wortes aufgenommen werden." (APRESJAN 1974b, 68)

3) Im EKW sollen - zumindest mit Hilfe der Sems - nur solche Wortschatzeinheiten definiert werden, die der abstrakten Lexik angehören:

"Einer semantischen Beschreibung soll vor allem die abstrakte Lexik unterzogen werden. Diese ist am aktivsten an der Paraphrasierung beteiligt." (MEL'CUK 1970, 216)

"Die Bedeutungen vieler Wörter (von Bezeichnungen für die 
Hauptarten von Tieren, Insekten, Pflanzen, Werkzeugen, industriell gefertigten Gegenständen und anderen materiellen objekten) können in einem erklärenden (nicht enzyklopädischen) Wörterbuch in den Termini der elementaren Bedeutungen nicht mehr mit hinreichender Vollständigkeit beschrieben werden. Zur Explikation solcher Wörter missen daher, ganz unabhängig davon, ob für sie enzyklopädische Erläuterungen gegeben werden oder nicht, auf jeden Fall im wesentlichen Abbildungen (ostensive definitions, définitions démonstratives) benutzt werden." (APRESJAN 1974b, 71)

4) Bei der Beschreibung synonymischer Paraphrasebeziehungen zwischen Sätzen im MST unterscheiden die Konstrukteure Paraphrasen, die Sprecher kraft ihres Sprachwissens vornehmen können, von Paraphrasen, denen ein bestimmtes Weltwissen von Sprechern zugrundeliegt. Im MST soll nur der erste Typ synonymischer Paraphrasen beschrieben werden:

Wir gehen von der Annahme aus, daB man die Angaben, die fiur eine Paraphrasierung erforderlich sind, in zwei Typen einteilen kann: in 'sprachliche Informationen' (alle möglichen Angaben uber Wörter und Konstruktionen.... die in den Kodex der Sprachbeherrschung eingehen) und in 'auBersprachliche Informationen' (Angaben uiber konkrete objekte und Gesetze in der realen Welt). Wir wollen die Bedeutung von solchen Sätzen als gleich ansehen, deren gegenseitige Substituierbarkeit man ausschließlich auf der Grundlage der Sprachbeherrschung ausmachen kann, ohne auBersprachliche Informationen uber die Welt heranzuziehen ..." (ZOLKOVSKIJ/MEL'CUK 1969, 13)

5) In der semantischen Beschreibung von Texten durch das MST sollen Sinnanomalien unbericksichtigt bleiben:

"Die Frage nach der Sinnhaftigkeit/Sinnlosigkeit (osmyslennost'/bessmyslennost') ... sprachlicher Ausdrücke beschäftigt uns nicht: iber die Zulässigkeit der einen oder anderen Kombination von Bedeutungen zu entscheiden, betrachten wir als eine Aufgabe, die möglicherweise die Grenzen der Zuständigkeit der Linguistik als solcher überschreitet... Unserer Ansicht nach soll sich die Linguistik in erster Linie mit der Frage beschäftigen, wie in einer gegebenen Sprache eine beliebige Kombination von Bedeutungen - unabhängig von ihrer Sinnhaftigkeit - ausgedruckt wird." (MEL'CUK 1966, 1342)

"Eine gegebene Bedeutung kann unserer Meinung nach eine falsche oder widersinnige Aussage enthalten oder sogar 
absolut sinnlos ... sein; für unser Modell ist das völlig unerheblich." (MEL'CUK 1974b, 21)

"Fragen der Sinnanomalie, Sinnhaftigkeit, Sinnlosigkeit, Widersprüchlichkeit, Trivialität usw. des Inhalts sprachlicher Ausdrücke überschreiten - obwohl sie für sich genommen äuBerst wichtig sind - den Rahmen unseres Modells. Wir glauben nicht, daB die Konstruktion von Mechanismen, die logische Beschreibungen von Bedeutungsinhalten leisten, eine eigentlich linguistische Aufgabe ist." (MEL'CUK $1974 b, 23$ )

Die oben genannten füf Annahmen sind wesentliche Bestandteile des Konzepts der Bedeutung sprachlicher Zeichen, von dem die Konstrukteure des MST in der semantischen Beschreibung ausgehen. Wir wollen sie im folgenden näher besprechen. Im Mittelpunkt unseres Interesses sollen die unter 1) - 4) genannten Annahmen stehen, da sie sich direkt auf die lexikographische Definition im EKW beziehen und somit insbesondere das Konzept der lexikalischen Bedeutung betreffen.

Zu 1):

In den lexikographischen Definitionen des EKW wollen die Konstrukteure des MST das "naive" Weltbild des Durchschnittssprechers (naivnaja kartina mira srednego nositelja jazyka) erfassen. Ist das "naive" Weltbild, das Sprecher mit einem gegebenen Lexem verbinden, expliziert, so ist dessen lexikalische Bedeutung im Rahmen des EKW mit hinreichender Vollständigkeit beschrieben. Dem "naiven" Weltbild des Durchschnittssprechers stellen die Konstrukteure des MST das "wissenschaftliche" oder "enzyklopädische" Weltbild (naučnaja/enciklopedičeskaja kartina mira) gegenüber. Obwohl sie sowohl das "naive" als auch das "wissenschaftliche" Weltbild als sprachliche Erscheinungen betrachten, soll letzteres jedoch nicht im EKW, sondern in speziellen fachsprachlichen Definitionswörterbiuchern beschrieben werden.

Die Konstrukteure des MST scheinen sich sehr sicher zu sein, in den lexikographischen Definitionen des EKW die Lexembedeutungen immer so zu explizieren, daB sie genau das "naive" Weltbild eines Durchschnittssprechers erfassen. Dies muissen wir ganz einfach deswegen annehmen, weil sich in ihren $\mathrm{Pu}-$ blikationen kaum ein Hinweis findet, $d a B$ eine Abgrenzung des 
"naiven" vom "wissenschaftlichen" Weltbild in den Bedeutungsexplikationen von Lexemen eventuell problematisch sein könnte (vgl.S. 110). Aufgrund der Homogenitätsannahme, von der die Konstrukteure des MST bei der semantischen Beschreibung ausgehen, deklarieren sie sich kurzerhand zu "Durchschnittssprechern" und geben ihr intuitives Wissen um die Bedeutungen der zu explizierenden Lexeme als das "naive" Weltbild aus. Untersucht man die lexikographischen Definitionen im Hinblick auf das Weltbild, das in ihnen expliziert wird, lassen sich etliche Beispiele finden, aus denen deutlich hervorgeht, daB die Konstrukteure des MST in ihnen uber das "naive" Weltbild des Durchschnittssprechers weit hinausgehen; vgl. die Bedeutungsexplikationen (25) - (27) von skorost', streljat' und kofe:

(25) skorost' $x-a-y=1$... velečina, charakterizujuščaja peremešcenie tela $i l i$ volny $x$ rasstojaniem $Y$, na kotoroe $X$ peremeščaetsja $v$ edinicu vremeni ...' (APRESJAN/ MEL'CUK/SALJAPINA 1973a, 3)

(26) X streljaet $v Y / \quad$ ' $X$ neposredstvenno kauziruet special'$v$ napravienii $k$ noe ustrojstvo 2 mgnovenno osvoboždat' $L-u$ iz $W-a$ Z-om = potencial'nuju ènergiju, kotoraja kauziruet 2 letet' iz $W-a$ napravienii $k L-u$, obyčno s cel'ju porazit' $y$.' (APRESJAN/ZOLKOVSKIJ/MEL'CUK 1975 ,

(27) $x-\underline{k o f e}=$ ' $X$ - vid kul'turnogo rastenija, prednanažennogo dlja polučenija iz ego plodov kofe 2a, - tropičeskoe derevo ili kustarnik semejstva marenovych.' (BOGDANOVA/NIKITINA/ MEL'CUK 1975, 3)

Man kann sich des Eindrucks nicht erwehren, daB die Konstrukteure des MST in (25) und (26) einen Physiker, in (27) einen Botaniker zu Wort kommen lassen. Welcher Durchschnittssprecher ist sich genau der Tatsache bewuBt, daB die Geschwindigkeit eines Gegenstands als das Verhältnis von zurückgelegter Wegstrecke ('rasstojanie $Y$, na kotoroe X peremesčăetsja...') und verstri- 
chener Zeit ('...v edinicu vremeni') definiert ist? WeiB er, daß auch eine Welle ('volna') eine bestimmte Geschwindigkeit hat? Entspricht es dem "naiven" Weltbild eines Durchschnittssprechers, die Kaffeepflanze in die Gruppe der Rötegewächse ('semejstvo marenovych') einzuordnen? ${ }^{28}$

Es ist ubrigens erstaunlich, daB die Konstrukteure des MST die lexikographischen Definitionen von skorost' und strezjat' als Explikation des "naiven" Weltbilds betrachten, die Bedeutungsexplikation (28) von zvuk jedoch mit der Behauptung verwerfen, sie gehe weit über das "naive" Weltbild (die "naive" Physik) hinaus:

(28) $x-\underline{z v u k}=' x-k o l e b a n i j a$ sredy s takoj-to dlinoj volny.' (APRESJAN 1974b, 75)

Unsere Behauptung, daB die Konstrukteure des MST in bestimmten lexikographischen Definitionen des EKW alles andere als das "naive" Weltbild des Durchschnittssprechers explizieren, wird im übrigen durch unsere empirische Untersuchung zur lexikographischen Tauglichkeit der SemS des EKW gestüzt. Bei der Dekodierung der lexikographischen Definition von jasnyj. in der die Konstrukteure des MST mit physikalischen und meteorologischen Termini nicht gespart haben, hatten unsere Versuchspersonen erhebliche Schwierigkeiten (vgl. S.252 f.).

Zweifellos haben die Konstrukteure des MST Recht, wenn sie von den lexikographischen Definitionen des EKW fordern, kein Spezialwissen zu beschreiben, das nur bestimmte Gruppen von Sprechern einer Sprachgemeinschaft über die Referenten eines zu explizierenden Lexems haben. Da sie das EKW als ein nichtfachsprachliches Definitionswörterbuch betrachten, können sie sich auf die Beschreibung des "naiven" Weltbildes beschränken und die Spezifizierung des "enzyklopädischen" Weltbilds anderen wissenschaftlichen Disziplinen überlassen. Den Konstrukteuren des MST muB jedoch der Vorwurf gemacht werden, daß sie so tun, als ob sie in der Lage wären, zumindest in den konkreten Bedeutungsbeschreibungen, die sie im EKW geben, die Grenze zwischen beiden Weltbildern ohne weiteres eindeutig zu ziehen. Auch wir können keine Kriterien nennen, nach denen eine solche Abgrenzung erfolgen könnte. Die Entschei- 
dung, ob eine Bedeutungsexplikation das "naive" oder das "wissenschaftliche" Weltbild eines Sprechers erfaBt, entspringt letztlich der Willkür des Lexikographen. Die Konstrukteure des MST sollten es jedoch tunlichst vermeiden, in den lexikographischen Definitionen des EKW fachsprachliche Ausdrücke zu verwenden, auch wenn das Bestreben nach Vollständigkeit der Bedeutungsexplikationen dies nahelegt.

\section{Zu 2)}

In den Wörterbuchartikeln des EKW sehen die Konstrukteure des MST, falls erforderlich, eine konnotative Zone vor, in der sie all diejenigen Bedeutungskomponenten auflisten, die sie zu der bzw. den Konnotation(en) des gegebenen Stichworts zählen. In dem Wörterbuchartikel von beispielsweise streljat' (in einer seiner Bedeutungen) führen die Konstrukteure des MST die folgenden Konnotationen an:

1) 'Gromkost', otryvistost' zvuka'

2) 'Rezkost', "otryvistost" "kratkovremennych aktov'

3) 'Bystrota peremě̌čenija'

(APRESJAN/ZOLKOVSKIJ/MEL'CUK 1975, 29)

Mit Hilfe der Konnotationen, die sie einem Stichwort neben dessen lexikalischer Bedeutung zuweisen, beschreiben die Konstrukteure des MST die Möglichkeit, das Wort in übertragener oder metaphorischer Bedeutung zu gebrauchen. Die drei oben genannten Konnotationen von streljat' werden beispielsweise in den Sätzen (29) - (31) in entsprechender Reihenfolge aktualisiert:

(29) Streljali zachlopyvajuščiesja kry̌̌ki part.

(30) X streljal v tolpu korotkimi zlymi frazami.

(31) X streljal glazami po storonam.

(APRESJAN/ZOLKOVSKIJ/MEL'CUK 1975, ebd.)

Da es sich bei den Erscheinungen, die die Konstrukteure des MST unter den Begriff der (lexikalischen) Konnotation subsumieren, um Inhaltskomponenten des zu explizierenden Lexems handelt, stellt sich zwangsläufig die Frage, wie diese Komponenten in begrüdbarer Weise bzw. - im Idealfall - in operationalisierbarer Weise von solchen Komponenten getrennt 
werden können, die der lexikalischen Bedeutung des gegebenen Lexems zugewiesen werden. Im Hinblick auf die Beispiele (29) - (31) verfügen die als Konnotationen bezeichneten Inhaltskomponenten von streljat, über die gleichen Eigenschaften, die auch diejenigen Inhaltskomponenten auszeichnen, die zur lexikalischen Bedeutung des Wortes gezählt wer. den (vgl. S. 83); bei beiden handelt es sich um überindividuelle, von der gesamten Sprechergemeinschaft vergesellschaftete Begriffe.

Während die Konstrukteure des MST bereits sehr früh die in ihren Extensionen einander nahestehenden Begriffe der lexikalischen Bedeutung und der grammatischen Bedeutung definieren (MEL'CUK 1961, 33-39 sowie ausführlicher 1974d, 95-113), findet sich erst relativ spät in ihren Veröffentlichungen eine befriedigende definitorische Abgrenzung des Begriffs der lexikalischen Bedeutung von dem Begriff der (lexikalischen) Konnotation (IOROANSKAJA/MEL'CUK 1980).

Als Ausgangspunkt für die Definition des Begriffs der Konnotation greifen IOROANSKAJA/MEL'CUK auf Oberlegungen zurück, die APRESJAN zu diesem Thema in APRESJAN 1974b, 67-68, anstellt (IORDANSKAJA/MEL'CUK 1980, 192). Unter der lexikalischen Konnotation eines Wortes versteht APRESJAN im Gegensatz zu ihrem üblichen Verständnis nicht subjektiv-wertende. emotional gefärbte Komponenten seiner Inhaltsseite, sondern solche Inhaltskomponenten,

"... die die mit einem Wort verbundenen kulturellen Vorstel. lungen und Traditionen wiedergeben, welche in der gegebenen Sprechergemeinschaft (obscestvo) in der Gebrauchspraxis der entsprechenden Sache dominant sind, sowie viele andere auBersprachliche Faktoren ... Diese Elemente gehören nicht direk: zur Semantik lgemeint ist: die lexikalische Bedeutung; $K . H$. des Wortes." (APRESJAN 1974b, 67)

APRESJANs sehr allgemein gehaltene Umschreibung des Begriffs der lexikalischen Konnotation dürte auf der Erkenntnis beruhen, daB die oft angefihrte Charakterisierung von Konnotationen als wertend-emotionaler Sprechereinstellungen kein taugliches definitorisches Kriterium ist, um die Konnotatio. nen von den lexikalischen Bedeutungen abzugrenzen, da sich 
im Wortschatz beliebiger natürlicher Sprachen viele Wörter finden, deren lexikalische Bedeutung aus wertenden bzw. emotionalen Komponenten zusammengesetzt sind; vgl. beispielsweise die deutschen und russischen Lexeme gut, schlecht, radovat'sja, nenavidet' (LORENZ/WOTJAK 1977, 127). Um zu einer Definition des Begriffs der lexikalischen Konnotation zu gelangen, knüpfen IORDANSKAJA/MEL' CUK an ein Beispiel an, das bereits in APRESJAN $1974 \mathrm{~b}$ im entsprechenden Zusammenhang kurz diskutiert wird. Ober die lexikalischen Konnotationen der Lexeme $\underline{i s a k}$ und $08 \ddot{e} z$ schreibt APRESJAN :

"Mit dem Wort išak wird beispielsweise die Vorstellung einer ergebenen Arbeitsbereitschaft assoziiert (vgl. rabotat, kak isak; chorosij isak; Ja vam ne isak tjanut, za vsech; Ne stanu isačit' za vsech), mit dem Wort osël, seinem genauen Synonym in der Hauptbedeutung, die VorsteTiung von Starrsinnigkeit und Dummheit (vgl. uprjamyj/glupyj kak osël; Nu $i$ osël že ty; Dovol'no os lit'!)." (APRESJAN 1974b, 67)

Da APRESJAN auf eine explizite, den Erfordernissen des EKW angemessene Darstellung der Inhaltsseite beider Stichwörter verzichtet, geben IORDANSKAJA/MEL'CUK zunächst deren vollständige lexikographische Definitionen samt den dazugehörigen konnotativen Zonen an; vgl. die Bedeutungsexplikationen $(32)-(33):$

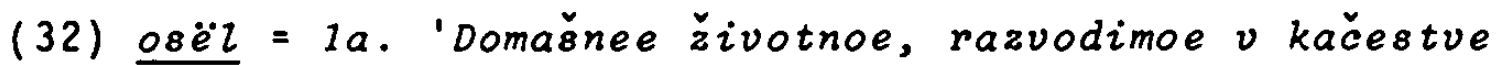
v'jučnogo ili uprjažnogo, - vysotoj v polovinu čelovečeskogo rosta, seroe, $s$ dinnymi usami i tonkim chvostom.'

1b. 'Samec osla 1a.' KONNOTACIJA: '(Uprjamo) glupyj.'

2. '(Uprjamo) glupyj [kak by osël 1b.] čelovek mužskogo pola.'

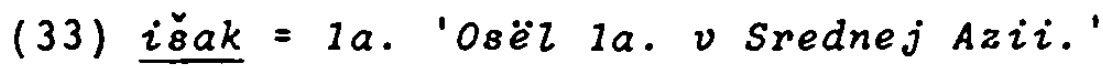
KONNOTACIJA: 'Bezropotno vypolnjajuščij tjaželuju ili dolguju rabotu. 29

1b. 'Samec ǐsaka la.'

2. 'Celovek, bezropotno vypolnjajuščij tjaželuju $i l_{i}$ dolguju rabotu [kak by išak la.].' (IORDANSKAJA/MEL'CUK 1980, 193-194) 
IORDANSKAJA/MEL'CUK weisen darauf hin, daB im Gegensatz zur gängigen Ansicht osëz 1b. bzw. osëz 2. über die ihnen zugewiesene Konnotation bzw. lexikalische Bedeutung keine dummen ('glupyj') oder starrköpfigen ('uprjamyj') Menschen bezeichnen. Es handele sich vielmehr um eine bestimmte, durch Starrköpfigkeit charakterisierte Art von Dummheit ('uprjamo glupyj'), die mit Hilfe von osël 1b. bzw. osël 2. bezeichnet werden kann; vgl. die entsprechende Verwendung von osël $1 b$. gegenuiber der von $\underline{d u b ~ 1 b}$. in den Sätzen (34) - (35):

(34) On formennyj osël. = 'Ego glupost' motivirovannaja NEŽLANIEM, prinimat' dovody $i$ razmyšljat', t.e. osobogo roda uprjamstvom.'

(35) On nastojaščij dub. = 'Ego glupost' motivirovannaja NESPOSOBNOST'JU prinimat' dovody i ră̌mysljat'.' (IORDANSKAJA/MEL'CUK 1980, 195)

Da sowohl der durch osël 1b. als auch der durch osëz 2. bezeichnete spezifische Typ von Dummheit jedoch nicht bei jeder Verwendung dieser Lexeme ausgedrückt werden muB ( $v g$ ). die Sätze (36) - (37)), wird die Inhaltskomponente 'uprjamo in den entsprechenden lexikographischen Beschreibungen der wörter durch Klammersetzung als fakultativ aktualisierbar ausgewiesen (s.o.):

(36) Ja vël sebja, kak osëz.

(37) A on, kak osël, vyložil vse den'gi srazu.

(IORDANSKAJA/MEL'CUK 1980 , ebd.)

Den Begriff der lexikalischen Konnotation definieren IORDANSKAJA/MEL'CUK wie folgt:

"Die lexika $l i s c h$ e $k$ o n not a $t i o n$ einer lexikalischen Einheit $L$ ist eine Charakterisierung, die L ihrem Referenten zuschreibt und die nicht zu deren Bedeutungsexplikation gehört." (IORDANSKAJA/MEL'CUK 1980, 196)

Zur Definition der lexikalischen Konnotation, in die bestimmte ihrerseits zu erläuternde Teilbegriffe eingehen, geben IORDANSKAJA/MEL' CUK die folgenden notwendigen Präzisierungen: 
1) Die oben genannte Definition soll ausschlieblich für lexikalische Konnotationen von Lexemen, nicht aber für enzyklopädische Konnotationen gelten. Unter enzyklopädischen Konnotationen verstehen IORDANSKAJA/MEL'CUK:

"... solche Konnotationen, die durch reale Eigenschaften des Referenten [einer lexikalischen Einheit; K.H.] bedingt ... und in keiner Weise an die eine oder andere Sprache gebunden sind." (IDRDANSKAJA/MEL'CUK 1980, 197)

Zu den enzyklopädischen Konnotationen rechnen sie Begriffe wie beispielsweise das hohe Gewicht einer Lokomotive oder eines Panzers oder die geringe Größe und Schutzlosigkeit eines Kindes.

Lexikalische Konnotationen bezeichnen sie dagegen als (einzel) sprachspezifische, an die Inhaltsseite ganz bestimmter Lexeme gebundene Erscheinungen, die sich unabhängig von den Eigenschaften ihrer Referenten konstituieren:

"Im Unterschied zu enzyklopädischen Konnotationen sind lexikalische Konnotationen nicht unbedingt an Gegenstände und Erscheinungen der physischen Welt gebunden. Sie setzen sich einfach an bestimmte lexikalische Einheiten fest, unabhängig davon, ob ihnen tatsächliche (oder nur mythische) Eigenschaften realer Gegenstände entsprechen ... Lexikalische Konnotationen sind für eine jede gegebene Sprache in besonderem Maße spezifisch." (IORDANSKAJA/MEL'CUK 1980, 198)

Obwohl sich sprachliche Vergleiche und Metaphern sowohl mit Hilfe enzyklopädischer als auch lexikalischer Konnotationen von Lexemen bilden lassen, soll nach IORDANSKAJA/MEL'CUK ausschlieBlich die Beschreibung lexikalischer Konnotationen in den Zuständigkeitsbereich der Linguistik fallen, da es nur vermittels dieses Typs von Konnotationen möglich sei, durch die Sprache kodifizierte Vergleiche zu ziehen. Für IORDANSKAJA/MEL'CUK handelt es sich beispielsweise bei den Sätzen (38) - (39) um einen durch die Sprache kodifizierten und daher durch eine lexikalische Konnotation bedingten Vergleich, während in Satz (40) ein freier, durch die Sprache nicht kodifizierter und daher durch eine enzyklopädische Konnotation motivierter Vergleich gezogen wird:

(38) Vsja pervaja glava ego dissertacii - čistaja vodal (39) Ty nužna mne, kak vozduch! 
(40) Ty nužna mne, kak voda umirajuščemu ot žaždy putniku $v$ pustyne.

(IORDANSKAJA/MEL'CUK 1980, 196, 197)

IORDANSKAJA/MEL'CUK begründen ihr Urteil damit, daB in (38) und (39) die für die lexeme voda und vozduch spezifischen lexikalischen Konnotationen 'bessoderžatel'nost' 'bzw.

'krajnjaja neobchodimost' ' realisiert werden, während in (40) eine für das Lexem voda nicht spezifische Inhaltskomponente - die wichtige Rolle, die das Wasser für die Aufrechterhaltung des menschlichen Lebens spielt - aktualisiert wird. Um zu zeigen, daB in (40) ein durch die Sprache nicht kodifizierter, auf einer enzyklopädischen konnotation von voda basierender Vergleich gezogen wird, bringen IORDANSKAJA/ MEL'CUK den Satz analog zu (38) und (39) in die Form eines im Russischen kodifizierten Vergleichs; vgl. Satz (40'): $\left(40^{\prime}\right)^{*}$ Ty nužna mne, kak voda!

Die Nichtwohlgeformtheit, die die elliptische form (40') gegenuiber Satz (40) aufweist, führen sie darauf zurück, daß trotz gewisser gemeinsamer, realer Eigenschaften der durch voda und vozduch bezeichneten Referenten im Russischen nur das Lexem vozduch, aber nicht voda ubber die mit der Bedeutung von nužna kompatible spezifische lexikalische konnotation 'krajnjaja neobchodimost' ' verfügt:

"Obwohl für das menschliche Leben das Wasser nicht weniger notwendig als die Luft ist, hat das russische Lexem voda (im Unterschied zum Lexem vozduch) nicht die Konnotation 'krajnjaja neobchodimost'." (IORDANSKAJA/MEL'CUK 1980, 198)

IORDANSKAJA/MEL'CUK illustrieren die einzelsprachliche Idiosynkrasie, die lexikalische Konnotationen von enzyklopädischen Konnotationen abgrenzt (s.o.), anhand mehrerer Beispiele aus ISACENKO 1972. Beispielsweise haben das russische Lexem koza 1b. = 'Samka kozy 1a.' und das deutsche Lexem ziege $1 b .=$ 'Weibchen von ziege $1 a$.' bei identischen lexikalischen Bedeutungen völlig verschiedene lexikalische Konnotationen. Während mit koza 1b. die lexikalische Konnotation 'črezmernaja podvižnost', prygučest' verbunden ist, hat ziege $1 b$. die lexikalische Konnotation 'črezmernaja 
govorlivost' (o ženščinach)'. In gleicher Weise verhalten sich die Inhaltsseiten des russischen Lexems vol und des tschechischen Lexems $\underline{v \ell l}$ zueinander; bei identischen lexikalischen Bedeutungen hat vol die lexikalische Konnotation 'terpelivoe trudoljubie', vil dagegen die lexikalische Konnotation 'intelzektual'naja tupost' ' (IORDANSKAJA/MEL'CUK 1980, 198).

Ein weiteres Beispiel für den einzelsprachspezifischen Charakter lexikalischer Konnotationen stellen die fast genau synonymen russischen Lexeme $\underline{0 s e ̈ l ~} 1$ und $\underline{i s a k 1}$ dar, die über zwei völlig verschiedene Konnotationen verfügen (s.0.).

Es ist zu vermuten, daB die Trennung, die IORDANSKAJA/MEL'CUK zwischen lexikalischen und enzyklopädischen Konnotationen vornehmen, keine dichotomische, sondern eher eine komparative Unterscheidung ist. Analog zur Problematik der Unterscheidung von "naivem" und "enzyklopädischem" Weltbild (vgl. S.94 ff.) sowie von Sprach- und Weltwissen in der Bedeutungsexplikation von Lexemen ( $v g l$. S. $110 \mathrm{ff}$. ), dürten sich etliche Fälle konstruieren lassen, in denen die Entscheidung, ob ein sprachlicher Vergleich kraft einer lexikalischen Konnotation oder einer enzyklopädischen Konnotation bezogen wird, willkürlich gefällt werden muB.

2) Im Zusammenhang mit der oben genannten Definition der lexikalischen Konnotation, die diese - sozusagen ex negativo als diejenigen Komponenten der Inhaltsseite eines Lexems bestimmt, die nicht zu dessen lexikalischer Bedeutung gehören und daher auch nicht in die Bedeutungsexplikation eingehen, weisen IORDANSKAJA/MEL'CuK darauf hin, daB zwischen dem Begriff der lexikalischen Konnotation und dem Begriff der lexikalischen Bedeutung in der Regel eine enge definitorische Beziehung besteht:

"Für eine gegebene lexikalische Einheit L kann dann eine lexikalische Konnotation $C$ postuliert werden, wenn in der gegebenen Sprache eine solche lexikalische Einheit $L$ ' existiert, in deren Bedeutungsexplikation es eine Komponente gibt, die völlig oder partiell mit dieser hypothetischen Kgnnotation zusammenfällt." (IORDANSKAJA/MEL 'CUK 1980, 199)

Eine vollständige Deckungsgleichheit einer Komponente des signifiés der lexikalischen Einheit L, die als deren lexi- 
kalische Konnotation betrachtet wird, mit einer Komponente des signifiés der lexikalischen Einheit $L^{\prime}$, die als deren lexikalische Bedeutung angesehen wird, liegt beispielsweise im Fall der (durch eine Derivationsrelation verbundenen) Lexeme veter und vetrenyj vor; vgl. die semantischen Beschreibungen (41) - (42):

(41) veter $=' \ldots$ '; KONNOTACIJA: 'Nepostojannyj'

(42) $\underline{\text { vetrenyj }}=$ 'Nepostojannyj (čelovek)' (IORDANSKAJA/MEL'CUK 1980 , ebd.)

Eine partielle Inklusion der entsprechenden (komplexen) Inhaltskomponenten illustrieren IOROANSKAJA/MEL'CUK am Beispiel der (in Polysemierelation stehenden) Lexeme osël $1 b$. und osël 2.; vgl. die semantischen Beschreibungen (43) - (44):

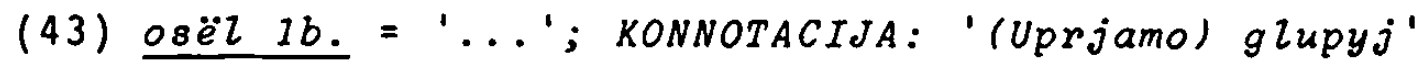

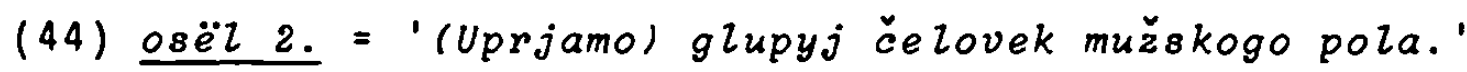
(IOROANSKAJA/MEL'CUK 1980, ebd.)

Der definitorische Bezug, in den IORDANSKAJA/MEL'CuK den Begriff der lexikalischen Konnotation und den Begriff der lexikalischen Bedeutung bringen, muB im Zusammenhang mit dem nichtoperationalen Charakter ihrer Definition der lexikalischen Konnotation gesehen werden, der sich seinerseits aus den bereits angesprochenen methodisch-methodologischen Problemen ergibt, die introspektives Vorgehen bei der Deskription fuir die linguistische Semantik aufwirft (vgl. S. $35 \mathrm{ff}$. ). IORDANSKAJA/MEL'CUK unterstreichen, daB sie über keine

"... formalen Kriterien verfügen, um die Frage zu entscheiden, ob ein gegebenes semantisches Element, das an eine le$x i k a l i s c h e$ Einheit $L$ gebunden ist, Teil deren Bedeutungsexplikation oder deren Konnotation ist." (IORDANSKAJA/MEL'CUK 1980,200 )

Aus diesem Umstand folgt für IOROANSKAJA/MEL'CUK, daB

"die Definition (der lexikalischen Konnotation (s.o.); K.H. nicht operational ist: sie gibt keine effektive Prozedur an, mit deren Hilfe hinsichtlich eines jeden semantischen Elements, das an eine lexikalische Einheit gebunden ist, entschieden werden kann, ob es als Komponente ihrer Bedeutungsexplikation oder ihrer Konnotation angesehen werden muB. Eine solche Prozedur braucht auch nicht, wie wir glauben, Inhalt einer Definition zu sein. Letztere hat die Aufgabe, eine Klas- 
se von Gegenständen zu kennzeichnen, indem sie deren wesentliche Eigenschaften angibt und für diese Klasse eine passende Bezeichnung vorschlägt. Die Frage, auf welche Weise man aufdecken kann, ob diese Eigenschaften bei den uns interessierenden Gegenständen vorhanden sind, ist ein ganz anderes Problem." (IORDANSKAJA/MEL'CUK 1980, ebd.)

Kann nicht auf operationale Weise bezijglich einer gegebenen Inhaltskomponente entschieden werden, ob sie der lexikalischen Bedeutung der betreffenden Einheit oder deren lexikalischer Konnotation - den standardisierten Assoziationen, die die Einheit im SprecherbewuBtsein hervorruft (IORDANSKAJA/MEL'CUK 1980, 200) - zuzuweisen ist, liegt es nahe, diese Inhaltskomponente in der Beschreibung zweimal anzugeben. Zu diesem Zweck spalten IORDANSKAJA/MEL'CUK die zu untersuchende lexikalische Einheit durch Mehrfacheintrag auf und fixieren die betreffende Inhaltskomponente einmal in der konnotativen Zone und einmal in der Bedeutungsexplikation der auf diese Weise gewonnenen Einheiten (s.o.).

3) Der definitorische Bezug, in den IORDANSKAJA/MEL'CUK den Begriff der lexikalischen Konnotation und den Begriff der lexikalischen Bedeutung bringen, veranlabt sie auBerdem, das Verhältnis von lexikalischer Konnotation und lexikalischer Polysemie zu präzisieren. Ausgangspunkt ihrer oberlegungen ist MEL'CUKs Konzeption der lexikalischen Polysemie, demzufolge zwei Einheiten mit gleichen signifiants dann in Polysemierelation stehen, wenn ihre Bedeutungsexplikationen einen gemeinsamen nichttrivialen Anteil haben (MEL'CUK 1974b, 110). Einen solchen Anteil konstatieren. IORDANSKAJA/MEL'CUK beispielsweise in den Bedeutungsexplikationen der Lexeme eerdce 1 und serdce 4, die sie daher als polyseme Einheiten betrachten; vgl. die unterstrichenen Semkomplexe in den lexikographischen Definitionen (45) - (46):

(45) $\underline{\text { serdce }}=1$. 'Vnutrennij organ čeloveka $x$, raepoložennyj $v$ central'-noj časti tela $X-a$ i imejuščij formu $\boldsymbol{C}$, - glavnyj organ krovoobraěčenija. KONNOTACIJA: 'V serdce $X-a$ nachoditsja organ čustv $X-a . '$ 
(46) $\underline{\text { serdce }}=4$.

'Central'naja čast' dostatočno bol's sogo učastka suši $x$, vydelennogo geografičeski ili administrativno.'

(IORDANSKAJA/MEL'CUK 1980, 207, 203)

Im Hinblick auf die Festlegung von lexikalischer Polysemie entsteht für IORDANSKAJA/MEL'CUK dann ein Problem, wenn sich bei zwei ausdrucksgleichen Lexemen zwar ein gemeinsamer, als nichttrivial eingestufter Anteil deren Inhaltsseiten ausmachen läßt, dieser jedoch einmal in der Bedeutungsexplikation und einmal in der konnotativen Zone dieser Lexeme erfaßt wird, wie es beispielsweise bei den wörtern serdce 1 (s.0.) und serdce 2 der Fall ist; vgl. die in der Bedeutungsexplikation (47) unterstrichenen Komponenten mit der konnotativen Zone in (45):

(47) $\underline{\text { serdce }}=2$. 'Organ čuvstv $X-a$.' (wie in: Serdce raduetsja.) (IORDANSKAJA/MEL'CUK 1980, ebd.)

IORDANSKAJA/MEL'CUK weisen darauf hin, daB in diesem Fall "... serdce 1 und serdce 2, die nur über die Konnotation miteinander verbunden sind, als Homonyme erscheinen könnten, was zweifellos der Intuition widerspricht." (IORDANSKAJA) MEL'(UK 1980, ebd.)

Um die oben genannte Konzeption des Begriffs der lexikalischen Polysemie nicht ändern und somit Lexeme wie beispielsweise serdce 1 und serdce 2 trotz der zwischen diesen intuitiv bestehenden Verbindung ihrer signifies nicht als Homonyme klassifizieren zu müssen, schlagen IORDANSKAJA/MEL'CUK für die hier in Rede stehenden fälle eine Modifikation der Struktur der Bedeutungsexplikation vor:

"Hat die lexikalische Einheit L die Konnotation ' $\alpha$ ' und die lexikalische Einheit $L^{\prime}$ ' in ihrer Bedeutungsexplikation die Komponente ' $a$ ", die völlig oder partiell mit ' $a$ ' zusammenfällt, so muB die Bedeutungsexplikation von L unbedingt auch eine Komponente 'kak by ... $L$ ' enthal ten." (IORDANSKAJA/ MEL'CUK 1980, 204)

IORDANSKAJA/MEL'CUK demonstrieren die veränderte Struktur der lexikographischen Definition u.a. an den folgenden Beispielen; vgl. die (unvollständigen) semantischen Beschreibungen (48) - (52) sowie (32) und (33) (5.0.): 
(48) $\underline{\text { serdce }}=1$.' 'Vnutrennij organ čeloveka $X \ldots$..'

KONNOTACIJA: ' $V$ serdce naohoditsja organ čustv $X-a . '$

(49) $\underline{\text { serdce }}=2$. 'Organ čuvstv $x-a$ [kak by nachodjaščijsja $v$ serdce 11 .'

(50) veter $=$ KONNOTACIJA: 1. 'Nepostcjannyj'

2. 'Bystro pereměsčajuščijsja'

(51) $x$ - vetrenyj $=$ ' $x$ - nepostojannyj [kak by veter] čelovek.'

(52) $x-a$ kak vetrom $8 d u z_{0}=' x$ črezvyc̆ajno bystro udalizsja

[kak by unesënnyj vetrom].

(IORDANSKAJA/MEL 'CUK 1980, ebd.)

Die deskriptive Aufgabe der Komponente 'kak by ...' erblicken IORDANSKAJA/MEL'CUK darin, daB sie einen besonderen Aspekt der lexikalischen Bedeutung des zu untersuchenden Lexems beschreibt, der - obwohl nicht für Referenzzwecke einsetzbar so etwas wie die "innere Form", die bildliche Struktur (obraznaja struktura) oder die semantische Etymologie des Wortes angibt.

Im Gegensatz zur konnotativen Zone entspricht dem modalen Rahmen kein eigenständiger Bereich im Wörterbuchartikel, da er, falls erforderlich, in die Bedeutungsexplikation des Stichworts eingebracht wird. Unter dem modalen Rahmen verstehen die Konstrukteure des MST in Anlehnung an WIERZBICKA die Einschätzung der von einem gegebenen Stichwort denotierten auBersprachlichen Situation durch den Sprecher oder Hörer (vgl. auch S. 92 ). Mit Hilfe des modalen Rahmens explizieren sie beispielsweise die Adverbien daže und tol'ko (in seiner "modalen" Bedeutung - eine Explikation von tol'ko in der Bedeutung 'isključitel'no' findet sich in MEL'CUK 1974b, $55 \mathrm{ff.}$ ) und das Adjektiv celyj; vgl. die Bedeutungsexplikationen (53) - (55):

(53) Daže A dejstvoval = 'Drugie dejstvovali, $i$ A dejstvuet, i govorjaščij ne ǒ̌idal, ŏto A budet dejstvovat'.'

(54) celyj $x$ (wie in: On s-el

celych dve tarelki.) =' $X, i$ govorjaščij š́itaet, čto 
èto mnogo.'

(55)

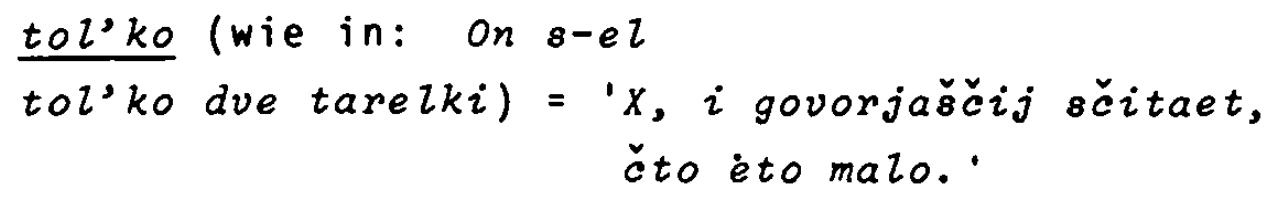

Die drei oben genannten Bedeutungsexplikationen zeigen im übrigen deutlich, daB die Trennung zwischen Wort- und Satzsemantik nicht aufrechterhalten werden kann. Eine Explikation der drei Lexeme, die diese nicht in ihrer Satz-oder Kontextform untersucht, wäre unmöglich (vgl. S. 65).

\section{$\mathrm{Zu}$ 3)}

Die Konstrukteure des MST führen zwei Oberlegungen an, die sie dazu veranlassen, nur Wortschatzeinheiten semantisch zu beschreiben, die der sogenannten abstrakten Lexik angehören. Der Terminus "abstrakte Lexik" muB in diesem Zusammenhang wohl als eine abkurzende Redeweise angesehen werden, da Wortschatzeinheiten mit abstrakten Referenten gemeint sind.

Die erste Oberlegung der Konstrukteure des MST stiutzt sich auf die Intention des MST, synonymische Paraphrasebeziehungen zwischen Texten zu beschreiben (vgl. S. 3). Bereits 1964 weist ZOLKOVSKIJ darauf hin, daß die abstrakte Lexik in Texten im Gegensatz zu deren konkreter Lexik an synonymischen Paraphrasen in sehr hohem Maße beteiligt ist (vgl. auch $\$$. 111). Gleichzeitig präzisiert er den Kreis der Wörter, die kraft ihrer Bedeutungen zur abstrakten Lexik zu rechnen sind:

"Nicht alle Wörter sind für die Beschreibung von gleichem Interesse ... die Semantik kann sich (wenigstens zu Anfang) auf Wörter mit abstrakten Bedeutungen konzentrieren. Das sind Wörter mit der Bedeutung Zeit, Grund, Ziel, Kontakt, Besitz, Wissen, Gefühl, Wille u. ä. Gerade diese Wörter können als Ergebnis semantischer Operationen [d.h. Synonymischer Paraphrasen; K.H.] ineinander umgeformt werden, ja sogar völlig in einem neuen Text aufgehen, während Wörter, die konkrete Gegenstände bezeichnen, bei allen Umformungen unverändert bleiben." (ZOLKOVSKIJ 1964, 6)

ZOLKOVSKIJs Vorschlag, den Bereich der abstrakten Lexik 
von dem Bereich der konkreten Lexik abzugrenzen, indem man Lexeme im Hinblick auf ihren Anteil untersucht, den sie an synonymischen Paraphrasen in einer gegebenen Sprache haben können, kann jedoch nur unter einer bestimmten, von ihm wohl implizit gemachten Voraussetzung realisiert werden. Diese besteht darin, daB es möglich sein muB, bezüglich einer jeden als synonymisch eingestuften Paraphrase zu entscheiden, ob sie auf sprachlichen Informationen oder dariber hinaus auf zusätzlichen nichtsprachlichen Informationen - dem sogenannten Weltwissen (vgl. S.94 f.) - beruht (SCHMIDT 1981).

Die zweite Oberlegung der Konstrukteure des MST bezieht sich auf die SemS des EKW und das ihr zugrundeliegende Konzept, Wortbedeutungen letztlich auf eine relativ kleine Anzahl von elementaren Bedeutungen zu reduzieren (vgl. S. $13 \mathrm{ff}$. ). Die Absicht der Konstrukteure des MST, sich aus den beiden oben genannten Gründen in der semantischen Deskription auf die abstrakte Lexik zu beschränken, wird durch die lexikographischen Beschreibungen, die sie im EKW für den russischen Wortschatz geben, eindeutig unterlaufen. Die Lexeme kofe, čaj, sosud, čajnik, skorost' (in der technischen Bedeutung 'Gang'), korobka skorostej, termometr, osël, isak, eerdce und golova (BOGDANOVA/NIKITINA/MEL'CUK 1975; APRESJAN/ ZOLKOVSKIJ/MEL'CUK 1975; APRESJAN/MEL'CUK/PERCOV/SALAPINA 1973b; IORDANSKAJA/MEL'CUK 1980) können sicherlich nicht als Wortschatzeinheiten angesehen werden, die zur abstrakten Lexik gehören.

Die Position, die die Konstrukteure des MST zur abstrakten und konkreten Lexik einnehmen, läBt die Grenzen deutlich werden, die der semantischen Beschreibung von Wortschatzeinheiten mit Hilfe der SemS gesetzt sind. Die Tatsache, daB die Konstrukteure des MST vorschlagen, für die Definition von Wörtern der konkreten Lexik im EKW U.a. Abbildungen zu verwenden, ist Ausdruck des engen Zusammenhangs, der zwischen der Beschränkung einer gegebenen semantischen Metasprache auf eine geringe Anzahl von Einheiten und dem damit einhergehenden Bedurfnis nach extensionalen oder 
ostensiven Definitionen besteht. Partiell extensionaler Definitionen, d.h. der Nennung einiger typischer Referenten, auf die man sich mit Hilfe des zu explizierenden Wortes beziehen kann, bedienen sich die Konstrukteure des MST übrigens in einigen Bedeutungsexplikationen des EKW; Vgl. die Spezifizierung des Sems 'vešcestvo' durch 'tabak' in der Explikation von kurit' und des Sems 'častica' durch 'dym, py $z$ ', tuman, oblaka' in der Explikation von jasnyj (vgl. S. 90, 217 ). Die einzige im Sinne der Konstrukteure des MST partiell ostensive, d.h. Abbildungen verwendende Definition findet sich in der Bedeutungsepxlikation von serdce 4 (vgl. S. 105).

Zu 4)

Um den Unterschied zwischen synonymischen Paraphrasen zu verdeutichen, die sich auf der Grundlage des Sprach-bzw. des Weltwissens von Sprechern vornehmen lassen, führen die Konstrukteure des MST u.a. die folgenden Beispiele an:

(56) Vse znajut, čto nemeckij narod boretsja s fašizmom.

(56') Vsem izvestno, čto narod Germanii vedët bor'bu protiv fašizma.

(56") My znaem, čto potomki Tomasa Mjuncera vstajut na puti gitzerovcev.

(57) On togo menija, čto griby polezny.

(57') On polagaet, čto griby polezny.

(58) Bolee poloviny territorii Egibty zanimajut pustyni. (58') I Egibte pustyni zanimajut ploščad', bol'šsuju čem vsja Erancija. (ZOLKOVSKIJ/MEL'CUK 1969, 12-13)

Die Paraphrasebeziehungen zwischen (56) und (56') sowie (57) und (57') lassen sich nach Ansicht der Konstrukteure des MST ausschlieBlich mit Hilfe von Sprachwissen, die zwischen (56) und (56") sowie (58) und (58') ausschlieblich mit Hilfe von Weltwissen herstellen. Die Konstrukteure des MST weisen ausdricklich auf die Schwierigkeiten hin, die die $A b-$ grenzung von Sprach- und Weltwissen aufwirft, so daB sie oft gezwungen sind, willkïliche Entscheidungen zu treffen (ZOLKOVSKIJ/MEL'CUK 1969, 13). 
Es lassen sich in der Tat keine intersubjektiv nachvollziehbaren Kriterien anführen, um zu entscheiden, ob Sprecher die Paraphrasen zwischen Sätzen wie beispielsweise (59) und (59') kraft ihres Sprach-oder kraft ihres Weltwissens herstellen können:

(59) Barometr padaet.

(59') Pogoda stanovitsja chuže.

Im Zusammenhang mit der Trennung des Sprachwissens (oder der Bedeutung) vom Weltwissen (oder Sachwissen) stellen LORENZ/ WOTJAK die Frage zur Diskussion, ob es überhaupt zweckmäBig sei, den Versuch zu unternehmen, beide Erscheinungen voneinander abzugrenzen. LORENZ/WOTJAK weisen darauf hin, daB die lexikographische Praxis in Ermangelung von geeigneten Trennungskriterien die beiden Bereiche nicht auseinanderhalte. Zwischen Sprach- und Weltwissen konstatieren sie auBerdem fließende Obergänge, da in der sprachlichen Kommunikation neben Bedeutungselementen auch Bestandteile des Sachwissens evoziert werden können. Aus diesen Gründen plädieren LORENZ/WOTJAK dafür, die Trennung von Sprach- und Weltwissen bzw. - in ihrer Terminologie - von Bedeutung und Sachwissen völlig aufzugeben und Bedeutungen als spezifische Form von kommunikativ verwendbarem, standardisiertem und vergesellschaftetem Sachwissen anzusehen (LORENZ/WOTJAK $1977,199 \mathrm{ff.}$.).

Aus den semantischen Beschreibungen, die die Konstrukteure des MST im EKW für russische Lexeme geben, wird deutlich, daB diese hinsichtlich der Abgrenzung von Sprach- und Weltwissen eine ähnliche Position wie LORENZ/WOTJAK beziehen, ohne sie jedoch explizit zu formulieren. In den lexikographischen Definitionen des EKW gehen die Konstrukteure des MST von einem bestimmten, willkürlich festgelegten MaB an gesichertem Weltwissen aus. Bei diesem gesicherten oder standardisierten Weltwissen handelt es sich um diejenigen Verwendungsweisen des zu explizierenden Lexems, die die Konstrukteure des MST als typisch betrachten. Sie abstrahieren von den typischen Verwendungsweisen des gegebenen Lexems, indem sie alle begrifflichen Komponenten festhalten und zu- 
einander in Beziehung setzen, die erforderlich sind, um dieses Referenzpotential des Lexems zu beschreiben. Die Bedeutung eines Lexems, die das EKW in seinen lexikographischen Definitionen - im Idealfall vollständig - explizieren soll, ist somit nichts anderes als eine Abstraktion der durch das Lexem typischerweise bezeichneten auBersprachlichen Situation (vgl. S. $90 \mathrm{f}$ ). Dies läßt sich am Beispiel der lexikographischen Definition, die sich im EKW für blagodarnyj findet, gut veranschaulichen. Die Konstrukteure des MST erachten es für erforderlich, um die durch blagodarnyj typischerweise bezeichnete auBersprachliche Situation zu beschreiben, in dessen Bedeutungsexplikation (60) die folgenden (unterstrichenen) Denotationsbedingungen aufzunehmen:

(60) X blagodaren $y-u z a z=$ 'Sèitaja, òto $y$ sdelal $x-u$ dobro Z, X čuvstvuet sebja objazannym kompensirovat' 2 slovesnym priznaniem ili otvetnym dobrym postupkom.' (APRESJAN 1974b, 107)

Es lassen sich selbstverständich Verwendungsweisen von blagodarnyj denken, in denen nicht auf Folgehandlungen referiert wird, die zwischen dem Mitspieler in der Agensrolle (' $X^{\prime}$ ) und dem Mitspieler in der Objektrolle (' $y^{\prime}$ ) stattfinden können. In der Bedeutungsexplikation (60) tragen die Konstrukteure des MST einer solchen Verwendung von blagodarnyj jedoch nicht Rechnung, weil sie sie nicht zur typischerweise durch das stichwort bezeichneten auBersprachlichen Situation zählen.

Man kommt in der linguistischen Semantik nicht umhin, ein gewisses MaB an gesichertem Weltwissen willkürlich festzulegen, indem man aus all den möglichen Verwendungsweisen eines Lexems bestimmte Realisierungen ausgrenzt, sie als typisch für das Referenzpotential des zu explizierenden Lexems ansieht und nur sie in der semantischen Beschreibung erfaBt. Würde man diese Abstraktion nicht vornehmen, so diufte die Anzahl ambiger Wortschatzeinheiten sehr stark ansteigen, da man gezwungen wäre, in die entsprechenden Bedeutungsexplikationen viele disjunktiv verknipfte Semkomplexe aufzunehmen. Auch 
würde sich das Problem, geeignete Selektionsbeschränkungen zu formulieren, in noch massiverer Weise als bereits gegenwärtig in der Linguistik stellen. Es bleibt fraglich, ob diese Wortschatzeinheiten dann noch im Rahmen einer linguistischen Semantik beschrieben werden könnten.

\section{Zu 5)}

Die Konstrukteure des MST formulieren ihre Position, bei der semantischen Beschreibung Sinnanomalien unberücksichtigt zu lassen, im Hinblick auf das MST als Grammatikmodell. Sie wollen im MST keine Filter vorsehen, die die Synthese von Sätzen wie beispielsweise (61) und (62) blockieren, voraus gesetzt diese sind mit der eingegebenen sinnanomalen semantischen Repräsentation synonymisch:

(61) Ivan uletel na Veneru.

(62) Ulybka poprekala kosmos černilami. (MEL'CUK 1974b, 22, 25)

Die Auffassung, die die Konstrukteure des MST zur Behandlung von Sinnanomalien im MST vertreten, steht allerdings im Widerspruch zu ihrem deskriptiven Vorgehen im EKW. In den Rektionsmodellen, mit denen die Stichwörter in ihren wörterbuchartikeln versehen werden, geben sie für die Realisierungsmöglichkeiten der verschiedenen Aktanten Selektionsrestriktionen mit Hilfe morpho-syntaktischer, semantischer und lexikalischer Informationen an (vgl. S. 116). Auf diese Weise werden nicht nur ungrammatische, sondern auch zum Teil sinnanomale Sätze ausgefiltert - letztere allerdings nur dann, wenn in der gegebenen Sprache zwei loder mehrere) synonyme Ausdrücke mit unterschiedlichen kombinatorischen Eigenschaften existieren (vgl. zum Verhältnis von Kombinierbarkeit/Idiomatizität und linguistischer Wohlgeformtheit APRESJAN 1978). 
2.2.2 Die Beschreibung der kombinatorischen Eigenschaften von Lexemen im erklärend-kombinatorischen Wörterbuch

Wie durch den Terminus "kombinatorisch" ausgewiesen, begreifen die Konstrukteure des MST das EKW nicht nur als ein definitorisches, sondern auch als ein kombinatorisches Wörterbuch oder Kollokationslexikon des Russischen. Sie untergliedern daher die Wörterbuchartikel des EKW in mehrere Zonen. Drei dieser Bereiche, die lexikographischen Definitionen des Stichworts, der illustrative Teil des Wörterbuchartikels (vgl. S. 72) und sein kontrastiver Teil (vgl. S. 72) verleihen dem EKW die Eigenschaft eines Definitionswörterbuchs.

Zwei weitere Zonen des Wörterbuchartikels, das Rektionsmodell des Stichworts (model' upravienija) und die Liste seiner lexikalischen Funktionen (Leksičeskie funkcii) machen das EKW zu einem kollokationswörterbuch.

Die Konstrukteure des MST bedienen sich des Rektionsmodells und der lexikalischen Funktionen, um das kombinatorische Potential oder die Kombinierbarkeit (sočetaemost', kombinatorika) eines gegebenen Stichworts sowohl im Hinblick auf dessen syntaktische als auch lexikalische Eigenschaften zu beschreiben :

"Es [d.h. das EKW; K.H.] wird als 'kombinatorisch' bezeichnet, weil es in erster Linie die kombinatorischen Eigenschaften von Wörtern - den Umfang ihrer syntaktischen und lexikal ischen Kombinierbarkeit - darstellen soll." (APRESJAN/ZOLKOVSKIJ/ MEL'CUK 1969, 2)

Dem Rektionsmodell fällt dabei die Beschreibung der syntaktischen Kombinierbarkeit des Stichworts $2 u$, worunter die Konstrukteure des MST die Erfassung

"... seiner syntagmatischen Eigenschaften im Bereich der grammatischen Bedeutungen ..." (APRESJAN 1968, 34)

verstehen. Die lexikalische Kombinierbarkeit des Stichworts, d.h. seine paradigmatischen und syntagmatischen Beziehungen, die es auf der Ebene der lexikalischen Bedeutungen zu anderen Lexemen eingehen kann, spezifizieren die konstrukteure des MST mit Hilfe der lexikalischen Funktionen.

Im folgenden wollen wir das Rektionsmodell und die lexikali- 
schen Funktionen kurz vorstellen. Bedingt durch die Zielsetzung unserer Arbeit, in deren Mittelpunkt die Untersuchung des EKW als Definitionswörterbuch steht, beschränken wir uns auf die Darstellung ihrer wichtigsten Charakteristika.

2.2.2.1 Das Rektionsmodell im erklärend-kombinatorischen Wörterbuch

In den Wörterbuchartikeln des EKW erhält jedes Stichwort (für jede seiner Bedeutungen) ein Rektionsmodell. Das Rektionsmodell hat zwei Aufgaben. Es soll einerseits die semantischen Valenzen (semantičeskie valentnosti) des Lexems, d.h. die Leerstellen für die Mitspieler oder Aktanten (učastniki, aktanty) der durch das Stichwort bezeichneten außersprachlichen Situation, ihren entsprechenden syntaktischen Valenzen zuordnen:

"Interessant für die theoretische Semantik und die Lexikographie sind die syntaktischen Eigenschaften des Wortes das sind in erster Linie seine aktiven semantischen Valenzen, d.h. diejenigen Valenzen des Wortes, die inm syntaktisch abhängige Wörter zuweisen und von denen jeder einer Variablen in seiner Bedeutungsexplikation entspricht. Weniger formal gesprochen kann man sagen, daB sich die semantischen Valenzen direkt aus der lexikalischen Bedeutung des Wortes ergeben und dieses als konkrete, von anderen verschiedene lexikalische Einheit charakterisieren. Die Inhalte oder 'Rollen', wenn man den Terminus von FILLMORE gebrauchen will, die ihnen zugeschrieben werden, ... sind Teile dieser lexikalischen Bedeutung." (APRESJAN 1974b, 119-120)

Andererseits soll das Rektionsmodell die Realisierungsmöglichkeiten der semantischen Valenzen des Wortes im Text unter morpho-syntaktischen, semantischen und lexikalischen Gesichtspunkten beschreiben.

Das Rektionsmodell eines Wortes besteht aus so vielen Kolumnen, wie es semantische Valenzen hat. Die Informationen über die Realisierungsmöglichkeiten der einzelnen semantischen Valenzen werden, sofern es sich um bestimmte morphosyntaktische Angaben handelt, innerhalb der einzelnen Kolumnen vermerkt. Handelt es sich um semantische oder lexikalische Angaben, so werden diese auBerhalb des Rektions- 
modells festgehalten. Anhand des (von uns partiell vervollständigten) Rektionsmodells von arendovat' wollen wir seinen Aufbau demonstrieren:

$A$ arenduet $u B-a C$ za $D$ na $T$ :

\begin{tabular}{|c|c|c|c|c|}
\hline $1=A-S u b$ & $2=C-O b j^{1}$ & $3=B$-Contrag & $4=0-o b j^{2}$ & $5=r$-Period \\
\hline$S_{\text {im. }}$ & $\begin{array}{l}S_{\text {vin. }} \\
\text { objazat. }\end{array}$ & $u s_{\text {rod. }}$ & za $S_{\text {vin. }}$ & $\begin{array}{l}\text { 1. na } S_{\text {vin. }} \\
\text { 2. srokom nad } \\
S_{\text {vin. }} .\end{array}$ \\
\hline
\end{tabular}

1) 1.: čelovek;

1.: oduševlënnoe sobiratel'noe

2) 2.: imena ugodij (les, učastok, ozero);

2. : imena kmupnych pomeščenij (zal, klub, saraj)

3) 4. deně̌naja summa (APRESJAN 1974b, 134)

Das Rektionsmodell des EKW weist deutliche Parallelen zu den Kasusrahmen (case frames) von FILLMORE und der Valenzbeschreibung deutscher Verben von HELBIG/SCHENKEL auf. In seiner oberen Querspalte beschreibt es die Kasusrollen oder semantischen Valenzen der verschiedenen Mitspieler, die an der durch das Stichwort bezeichneten auBersprachlichen Situation beteiligt sind. Ihre Anzahl und ihren Inhalt legen die Konstrukteure des MST intuitiv fest. Die gleichen Angaben finden sich - lediglich in anderer Schematisierung - in den FILLMOREschen Kasusrahmen; vgl. den Kasusrahmen von to zift ( $A$ steht für den Tiefenkasus 'Agent', 0 für 'Object'): $x$ lifts $Y$ :

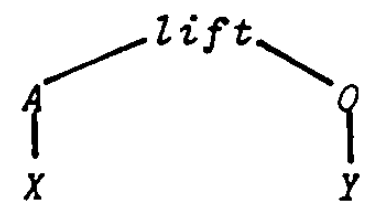

(FILLMORE 1969, 117)

Im Unterschied zur Kasusgrammatik FILLMOREs, die im höchsten Fall acht verschiedene Kasusrollen ansetzt, umfaBt das Inventar der Tiefenkasus, von dem die Konstrukteure des MST ausgehen, 25 verschiedene Kasusrollen (APRESJAN 1974b, 125126). Charakteristisch für das Konzept der Kasusrollen im 
Rektionsmodell des EKW ist es, daB der Tiefenkasus 'Subjekt' ('sub') sowohl den Agens einer Handlung (celesoobraznyj dejate l') als auch den Träger einer Eigenschaft (nositel' evojetva), der Tiefenkasus 'objekt' ('obj') nur ein von der Handlung affiziertes Objekt und der Tiefenkasus 'Resultat' ('Resu2t') nur ein von der Handlung effiziertes Objekt bezeichnen können, vgl. die Beispiele (63) - (65):

(63) Borisu $\stackrel{\text { sub }}{\longleftarrow}$ durno.

(64) Boris $\stackrel{S u b}{\stackrel{\text { Result }}{\longrightarrow}}$ pišet mo.

(65) Boris $\stackrel{S u b}{\longrightarrow}$ gladit $\stackrel{O b_{j}}{\longrightarrow}$ rubašku.

In der unteren Querspalte des Rektionsmodells werden bestimmte morpho-syntaktische Realisierungsmöglichkeiten der Kasusrollen mit Hilfe von entsprechenden Regeln beschrieben. AuBerdem finden sich (durch den Zusatz 'objazat.' (= 'objazatel'no') Angaben zur obligatorik bzw. Fakultativität der syntaktischen Realisierung der semantischen Valenzen. Die Spezifizierung der Realisierungsmöglichkeiten der Kasusrollen eines Wortes im Rektionsmodell des EKW entspricht der quantitativen und der qualitativen Valenzbeschreibung, die HELBIG/ SCHENKEL - allerdings nur für syntaktische Valenzen - auf der ersten und der zweiten Stufe ihres Valenzmodells vornehmen; vgl. die erste und die zweite Stufe des Wörterbuchartikels, den sie in ihrem Valenzwörterbuch dem Verb fragen (in einer seiner Bedeutungen) zuweisen:

\section{fragen}

I. fragen $_{1}+(2)=3$

II. fragen $\rightarrow s_{n}\left(s_{a}\right),\left(p S / N S_{o b, w .}\right.$

(HELBIG/SCHENKEL 1973, 103)

In speziellen Zusätzen auBerhalb des Rektionsmodells formulieren die Konstrukteure des MST Selektionsrestriktionen, denen die Auswahl der Lexeme unterliegt, die die im Rektionsmodell ausgewiesenen Kasusrollen einnehmen können. Sie zählen u.a. die semantischen Merkmale der Lexeme auf und oder definieren sie extensional durch Auflistung (vgl. S. $131 \mathrm{ff}$ ). Im Rektionsmodell von arendovat' werden beispiels- 
weise durch die Zusätze unter 2) den lexikalischen Realisierungsmöglichkeiten des Mitspielers in der Rolle des ersten Objekts Restriktionen auferlegt, die die Wortverbindung "arendovat" komnatu als ungrammatisch ausweisen (5.0.).

Die Konstrukteure des MST wollen das Rektionsmodell in der oben dargestellten Form in das EKW übernehmen. Es ist sehr zweifelhaft, ob ihr Anspruch, mit dem EKW ein im Vergleich zu gängigen Wörterbüchern effektiveres Kollokationslexikon des Russischen vorzulegen, dann noch aufrechterhalten werden kann. Die potentiellen Adressaten des Wörterbuchs müBten uber linguistische Spezialkenntnisse verfügen, um das Rektionsmodell eines Wortes verstehen zu können. Aus der Sicht eines potentiellen (nicht linguistisch gebildeten) Benutzers, der das EKW befragt, um Lexemkollokationen nachzuschlagen, ist es erforderlich, das Rektionsmodell zu modifizieren. Man sollte seine abstrakte und schematische Notationsform aufgeben und die morpho-syntaktischen Real isierungsmöglichkeiten der semantischen Valenzen des gegebenen Stichworts - analog zu gängigen russischen Wörterbüchern durch sogenannte Kasusfragen (padežnye voprosy; vgl. S. 181 ) beschreiben. Die Restriktionen, denen die semantische und lexikalische Realisierung der semantischen Valenzen unterliegt, sollte man ausschließlich extensional beschreiben, um nicht zu hohe Anforderungen an das Abstraktionsvermögen und die Sprachreflexion der potentiellen Wörterbuchbenutzer zu stellen. Zu diesem Zweck kann man auf den Vorschlag der Konstrukteure des MST zurückgreifen, im Wörterbuchartikel einen Bereich mit Beispielen für konkrete Realisierungen des Rektionsmodells vorzusehen, der auch sogenanntes negatives sprachliches Material, d.h. Beispiele grammatisch nichtwohlgeformter Sätze oder Phrasen mit dem gegebenen Stichwort umfaBt (s.o.):

"Unter den Beispielen der sprachlichen Realisierung des Rektionsmodells werden nicht nur mögliche und empfohlene Realisierungen angefuhrt, sondern auch speziell konstruierte Beispiele für unzulässige Realisierungen...., d.h. jenes 'negative sprachliche Material', auf dessen Einbezug in ein normatives Definitionswörterbuch schon Sčerba bestanden hat." (MEL'CUK 1974b, 112) 
2.2.2.2 Die lexikalischen Funktionen im erklärend-kombinatorischen Wörterbuch

Mit Hilfe der lexikalischen Funktionen erfassen die Konstrukteure des MST die lexikalische Kombinierbarkeit eines gegebenen Stichworts. Unter dem kombinatorischen Potential, über das ein Stichwort auf der Ebene der lexikalischen Bedeutungen verfügt, verstehen die Konstrukteure des MST sämtliche Wörter und Wortverbindungen,

"... die mit ihm auf eine bestimmte Weise semantisch verbunden sind, und zwar 1) seine 'paradigmatischen Varianten' oder $s u b s t i t u t i o n$ e $n$ (zameny), d.h. Ausdrucksmittel, die das stichwort in diesem oder jenem Kontext unter diesen oder jenen Bedingungen ersetzen können oder müssen; 2) seine 'syntagmatischen Partner' oder $P$ a $r$ a $m e$ $t e r$ (parametry), d.h. Ausdrucksmittel, die bei einem gegebenen Wort einige Bedeutungen idiomatisch, d.h. gebunden ausdrijcken." (MEL'CUK 1974b, 78)

Die Aufgabe, die die in zwei Typen - die Substitutionen und die Parameter - zerfallenden lexikalischen Funktionen im EKW zu erfüllen haben, besteht darin, daB die Substitutionen einem gegebenen Stichwort seine paradigmatischen Varianten zuweisen und die Parameter Realisierungsvarianten bestimmter Bedeutungen bei einem gegebenen Stichwort aufzählen. Die Konstrukteure des MST verwenden den Begriff der lexikalischen Funktion in Anlehnung an den mathematischen Funktionsbegriff. Eine lexikalische Funktion ordnet ihrem Argument, einem gegebenen Stichwort, bestimmte Lexeme oder Syntagmen als Wert (anačenie, vyraženie, leksičeskij korreljat) der Funktion zu. Da die lexikalischen Funktionen ausschließlich das kombinatorische Potential eines gegebenen Stichworts und nicht dessen Bedeutung beschreiben sollen (MEL'CuK 1974b, 78), können die Relationen, die verschiedene lexikalische Funktionen zwischen dem Stichwort und ihren entsprechenden Werten stiften, sowohl semantischer als auch syntaktischer Natur sein.

Die Konstrukteure des MST nehmen in das EKW nur solche lexikalischen Funktionen auf, deren Werte für verschiedene Stichwörter idiosynkratisch sind, da diese Werte als kombinatorisch bedingte Varianten des Ausdrucks derselben le- 
$x$ ikalischen Funktion bei verschiedenen Argumenten angesehen werden können (APRESJAN 1967, 14). Für die Beschreibung der lexikalischen Kombinierbarkeit von Lexemen sind nur diese lexikalischen Funktionen von lexikographischem Interesse:

"Von all den möglichen lexikalischen Funktionen wollen wir diejenigen ausgrenzen und im folgenden auch nur betrachten, die $p h r a s$ e $l o g$ i $s c h$ g e b u n d e $n$ e A u s d $r$ ij $c k$ e haben, d.h. solche lexikalischen Funktionen, die über Ausdrücke verfügen, welche bei bestimmten Argumenten zulässig sind, bei anderen jedoch nicht."

(MEL'CUK 1974b, 79)

Die Menge der lexikalischen Funktionen, deren Werte, bei den verschiedenen Stichwörtern, für die sie definiert sind, phraseologisch gebunden ausgedrückt werden, untergliedern die Konstrukteure des MST in sogenannte lexikalische Standardfunktionen und lexikalische Nichtstandardfunktionen (standartnye/nestandartnye leksičeskie funkcii). Um als Standardfunktion gelten zu können, muß eine lexikalische Funktion zwei Bedingungen genügen. Sie muB 1. für eine hinreichend groBe Anzahl von Argumenten definiert sein und 2. bezüglich der Werte, die sie bei verschiedenen Argumenten haben kann, über eine hinreichend große Vielfalt an Ausdrükken verfügen (MEL'CUK 1974b, 79-80). Die Konstrukteure des MST weisen ausdrücklich darauf hin, daB sie die quantitativen Entscheidungen, welche die definitorischen Bedingungen, die sie an die lexikalischen Standardfunktionen knüpen, erforderlich machen, nicht vollziehen können, so daB deren Abgrenzung gegenüber den lexikalischen Nichtstandardfunktionen vorerst willkürlich vorgenommen wird (MEL'CUK 1974b, 80).

Wir wollen im folgenden die lexikalischen Standarfdunktionen näher betrachten, da sie für das EKb! als Kollokationswörterbuch eine zentrale Rolle spielen (vgl. zu den lexikalischen Nichtstandardfunktionen MEL'CUK 1974b, 100-101). Die lexikalischen Standardfunktionen nennen wir der Kürze halber lexikalische Funktionen.

Die Konstrukteure des MST teilen die lexikalischen Funktionen in lexikalische Substitutionen (zameny) und in lexikalische Parameter (parametry) auf (s.0.). Die Werte der lexikalischen Substitutionen ersetzen das Argument, für das sie 
definiert sind, die Werte der lexikalischen Parameter werden mit dem Argument, für das sie definiert sind, syntagmatisch verbunden. Die lexikalischen Parameter, die den gröBten Teil der lexikalischeh Funktionen im EKW ausmachen, sind hinsichtlich ihrer Werte nicht homogen. Die Konstrukteure des MST unterscheiden bei den lexikalischen Parametern zwei Typen lexikalischer Korrelate:

7 "... solche, die gewöhnlich gleichzeitig die Bedeutung des Stichworts $c_{0}$ und eine zusätzliche ... Bedeutung abdecken, und solche, die gewöhnlich nur eine zusätzliche Bedeutung (oder sogar uberhaupt keine Bedeutung) ausdrücken, jedoch nicht die Bedeutung des Stichworts $C_{0}$." (MEL'CUK 1974b, 81)

Den beiden oben genannten Typen von lexikalischen Parametern entsprechen in der Regel unterschiedliche Realisierungsmöglichkeiten, mit denen ihre Werte bei den entsprechenden Argumenten auf der syntagmatischen Achse verknüpt werden. Die lexikalischen Parameter des ersten Typs werden bei einem gegebenen Argument gewöhnlich mit diesem zusammen in einem Lexem oder Syntagma ausgedrijckt. Die Konstrukteure des MST sprechen in diesem Zusammenhang von einem verschmolzenen Ausdruck (skzeenoe vyraženie) des Stichworts und des lexikalischen Korrelats des entsprechenden lexikalischen Parameters. Die lexikalischen Parameter des zweiten Typs werden gewöhnlich bei einem gegebenen Stichwort gesondert in Form eines nichtverschmolzenen Ausdrucks (neskleenoe vyraženie) realisiert.

Zu den lexikalischen Parametern, die in der Regel bei den Argumenten, für die sie definiert sind, einen verschmolzenen Ausdruck aufweisen, gehört u.a. die lexikalische Funktion $S_{i}$, welche einem gegebenen Stichwort die typischen Bezeichnungen für den $i$-ten Aktanten der auBersprachlichen Situation, die es bezeichnet, zuweist. Die Konstrukteure des MST zählen mit Hilfe der lexikalischen Funktion

$S_{i(i=1,2,3,4)}$ die typischen Bezeichnungen für sämtliche Aktanten auf, die an den auBersprachlichen Situationen, welche beispielsweise von den Lexemen prodavat' und lekcija bezeichnet werden, beteiligt sind. Das Symbol // vor dem jeweiligen Wert der lexikalischen Funktion weist auf den ver- 
schmolzenen Ausdruck ihres lexikalischen Korrelats hin:

$s_{1}($ prodavat') = // prodavec

$s_{2}$ (prodavat') = // tovar

$S_{3}$ (prodavat') = // pokupatel'

$s_{4}$ (prodavat') = // cena; plata (MEL'CUK 1974b, 87) $s_{1}($ lekcija) $=/ /$ lektor

$s_{2}(l e k c i j a)=/ / s$ lušatel'

$s_{3}($ lekcija) = tema; soderžanie

Aus den Werten, die die lexikalische Funktion $s_{i}$ bei den oben genannten Stichwörtern hat, fällt lediglich die typische Bezeichnung des dritten Aktanten, dem in der durch lekcija bezeichneten Situation die Inhaltsrolle ('Content') zukommt, heraus, da er einen nichtverschmolzenen Ausdruck hat; vgl. die Wortverbindung tema lekcii.

Zu den lexikalischen Parametern, die bei den Argumenten, für die sie definiert sind, einen nichtverschmolzenen Ausdruck haben, gehört u.a. die lexikalische Funktion Oper ${ }_{1,2}$. Sie gibt das Verb an, das die Bezeichnung des ersten bzw. des zweiten Aktanten, der an der auBersprachlichen Situation, die ihr Argument bezeichnet, beteiligt ist, als syntaktisches Subjekt mit der Bezeichnung der Situation, d.h. dem Argument selbst, als erstes syntaktisches objekt verknupft:

Oper $_{1}($ poddeř̌ka $)=$ okazyvat,

oper $_{1}$ (prepjatstvie) = činit.

Oper $_{1}(v$ lijanie $)$ okazyvat.

oper $_{2}$ (poddeř̌ka) =nachodit', vstrečat'

oper $_{2}$ (prepjatstuie) = stalkivat'sjas

oper $_{2}$ (viijanit) = byt' pod, nachodit'sja pod, ispytyvat', podvergat'sja (MEL'CUK 1974b, 94; APRESJAN 1974b, 45 , 46)

Die lexikalischen Funktionen können, wie bereits erwähnt, sowohl semantische als auch syntaktische Relationen zwischen Lexemen stiften. Als Beispiel für eine bedeutungshaltige lexikalische Funktion wollen wir die lexikalische Funktion Magn anfuhren. Ihre Werte geben als Magn ${ }_{0}$ einen hohen Grad an Intensität bzw. Ausprägung der Handlung bzw. des Zustands an, den ihre Argumente bezeichnen: 
Magn $(m o l \grave{c} a n i e)=$ polnoe, absoljutnoe; grobovoe Magn $\left(s p a t^{\prime}\right)=$ krepko; kak ubityj, bez zadnich nog Magn $_{0}(k r i c ̌ a t ')=$ gromko, izo vsech sil, vo vsë gorlo Magn $_{0}($ dožd') = kak iz vedra, prolivnoj, // liven' (MEL'CUK 1974b, 82, 89)

Magn $_{0}\left(z_{j u b i t}\right)=$ gorjačo, strastno Magn (ograblenie) = derzkoe, krupnoe (UBIN 1969, 61)

Die lexikalische Funktion Magn, spezifizieren die Konstrukteure des MST in bestimmten Fällen noch durch die Zusätze "temp" und "intens":

Magn ${ }_{0}^{\text {temp }}$ (aplodismenty) = prodolizitel'nye, nesmolkaemye Magn $_{0}^{\text {intens }}$ (aplodismenty) = burnye

(MEL'CUK 1974b, 102; vgl. für weitere Spezifizierungen von Magn。 UBIN 1969)

Beispiele für bedeutungsleere lexikalische Funktionen sind die lexikalischen Funktionen oper $1,2\left(s_{0.0}\right), F^{2} c_{0,1,2}$ und Labor $_{12}$. Sie weisen ihren Argumenten als entsprechende Werte Verben zu, die als rein syntaktische Verknüpfer zwischen dem jeweiligen Stichwort und den Bezeichnungen seiner verschiedenen Aktanten eingesetzt werden. Die lexikalische Funktion Func $_{0,1,2}$ weist ihrem Argument das Verb $z u$, das die Bezeichnung der Situation, d.h. das Argument selbst, als syntaktisches Subjekt mit den Bezeichnungen der Aktanten, die an der Situation beteiligt sind, verknupft, vorausgesetzt, sie sind uberhaupt fur das jeweilige Argument definiert:

Func ${ }_{0}\left(n o{ }^{\prime}\right.$ ' moroz) = stojat'

Funco $(t i$ sina $)=$ stojat' carit'

Func, $\left(d o \check{z} d^{\prime}\right)=i d t i$

Func $_{1}($ zasluga) = prinadlěrat'

Func, $($ prikaz) = ischodit' ot

Func $_{2}($ kara $)=$ postigat'

Func $_{2}($ (vast') = rasprostranjat'sja na (MEL'CUK 1974b, 94)

Die lexikalische Funktion Labor 12 weist ihren Argumenten das Verb zu, das die Bezeichnung des ersten Aktanten der Situation als syntaktisches Subjekt mit der Bezeichnung 
des zweiten Aktanten der Situation als erstes syntaktisches Objekt und der Bezeichnung der Situation, d.h. dem Argument selbst, als zweites syntaktisches objekt verknupft:

Labor $_{12}$ (nakazanie) = podvergat

Labor $_{12}$ (vlast') = imet' pod, imet' vo, deržat' pod Labor $_{12}$ (orden) = nagraždat' (MEL'CUK 1974b, 94)

Die Konstrukteure des MST haben bisher 40 lexikalische Funktionen definiert und zur Beschreibung der lexikalischen Kombinierbarkeit russischer Lexeme im EKW eingesetzt. Zur Benennung der lexikalischen Funktionen bedienen sie sich bestimmter lateinischer Abkürungen (s.0.). Die Konstrukteure des MST beabsichtigen, im EKW die lexikalische Kombinierbarkeit der Lexeme zu erfassen, indem sie für jedes stichwort uberprüfen, welche lexikalischen Funktionen für das Wort definiert sind und indem sie gegebenenfalls unter Verwendung der oben genannten Symbolik die entsprechenden lexikalischen Korrelate in dem Wörterbuchartikel auflisten:

"... die Beschrejbung der lexikalischen Kombinierbarkeit eines Wortes kann man sich als das Ausfijllen eines standardisierten Fragebogens vorstellen, dessen Fragen die Bezeichnungen typischer Bedeutungen [d.h. die lexikalischen Funktionen; K.H.] und dessen Antworten die Möglichkeiten ihres lexikalischen Ausdrucks bej einem gegebenen Wort in einer gegebenen Sprache bilden." (APRESJAN/Z̈OLKOVSKIJ/MEL'CUK 1969d, 65)

Da sich in den Arbeiten der Konstrukteure des MST keine anderen Angaben finden, sie im Gegenteil sogar die lexikalischen Funktionen in der oben angeführten Notationsweise für das EKW vorstellen, müssen wir annehmen, daß sie auch in dieser Form in seine Wörterbuchartikel eingehen sollen. Den potentiellen Adressaten, die das EKW befragen sollen, um Lexemkollokationen oder Einzellexeme nachzuschlagen, unterstellen sie daher ein auBerordentlich hohes AusmaB an theoretischen linguistischen Kenntnissen und Abstraktionsvermögen. Wie sollte es sonst einem russischen Muttersprachler möglich sein, die jeweiligen Definitionen der lexikalischen Funktionen zu verstehen und diese bei der Benutzung des Wörterbuchs mit den entsprechenden lateinischen Symbolen in Verbindung zu bringen. Es erscheint uns äuBerst zweifelhaft, 
ob der Anspruch, den die Konstrukteure des MST für das EKW geltend machen, als neuartiges kollokationswörterbuch eine bessere lexikographische Alternative zu gängigen russischen Wörterbüchern bieten zu können, unter diesen Umständen aufrechterhalten werden kann. Ein potentieller Benutzer des EKW, der sich zunächst einer Art linguistischem Propädeutikum unterziehen muB, um in die Definitionen und die Symbolisierungen der lexikalischen Funktionen eingeführt zu werden, wird bestimmt - vorausgesetzt, er ist kein Linguist - andere Wörterbiucher befragen, obwohl die Systematik, mit der sie Lexemkollokationen erfassen, im Vergleich zu der des EKW schlechter ist (s.u.). Die Unbedachtheit, mit der die Konstrukteure des MST den Anspruch erheben, die lexikalischen Funktionen in der oben dargestellten Form in das EKW zu übernehmen, ist ein weiterer Beleg für die bereits mehrfach festgestellte Tatsache, daB sie Eigenschaften seiner potentiellen Benutzer, die die Art und Weise der lexikographischen Präsentation der Wortschatzeinheiten wesentlich beeinflussen, völlig ignorieren. Der gleiche Vorwurf ist auch an REUTHER zu richten, der voller Euphorie die Tauglichkeit des EKW für die lexikographische Praxis herausstellt:

"Der Verfasser [d.h. REUTHER; K.H.] kann nur aus seiner Erfahrung berichten, daß er niemanden kennt, der ein solches Wörterbuch nicht besitzen möchte." (REUTHER 1978, 35)

REUTHER diurfte nur dann Recht haben, wenn er an den Linguisten als potentiellen Adressaten des EKW denkt.

In den Publikationen, in denen sie die lexikalischen Funktionen im Hinblick auf die zweisprachige (russisch-englische) Lexikographie und den mutter-bzw. fremdsprachlichen Unterricht vorstellen, vernachlässigen die Konstrukteure des MST die potentielle Zielgruppe, an die sie sich mit inren Wortschatzbeschreibungen wenden wollen, in ebenso rigoroser Weise. In APRESJAN/ZOLKOVSKIJ/MEL'CUK 1969b umreiBen die Konstrukteure des MST die Situation, in der ein zweisprachiges EKW von seinen potentiellen Adressaten befragt werden sollte: 
"... ein gebildeter Ausländer - beispielsweise ein Wissenschaftler oder Journalist -, der zwar nur über rudimentäre Russischkenntnisse verfiugt, jedoch ein klares Verständnis des Gedankens hat, den er mitteilen möchte, beginnt damit, ihn in mangelhaftes Russisch zu bringen; danach greift er zum EKW, das ihn befähigt, den zunächst groben Ausdruck der intendierten Bedeutung, der für einen Muttersprachler unbeholfen und nicht akzeptabel ist, in gutes idiomatisches Russisch zu übertragen." (APRESJAN/ZZOLKOVSKIJ/MEL'CUK 1969b, 2)

Sofern der "gebildete" Ausländer, der das zweisprachige (russisch-englische) EKW benutzen soll, nicht linguistisch "gebildet" ist oder sich mit den lexikalischen Funktionen und ihrer Notation nicht ausfürlich beschäftigt hat, durfte er jedoch mit dem wörterbuch nicht viel anfangen können.

In APRESJAN/ZOLKOVSKIJ/MEL'CUK l969d stellen die Konstrukteure des MST neben dem Rektionsmodell die lexikalischen Funktionen in der oben angefürten Notationsweise für die Wortschatzarbeit im mutter- und fremdsprachlichen Unterricht vor, ohne auf die Eigenschaften einzugehen, über die die Adressaten einer solchen Wortschatzbeschreibung verfügen sollen. Aus dem Artikel geht nicht deutlich hervor, ob sich die Konstrukteure des MST an Lehrer oder Lernende wenden. Ginge dem Aufsatz nicht ein Vorwort der Redaktion der Zeitschrift "Russkij jazyk v nacional'noj škole" voraus, in dem das Instrumentarium, mit dem die Konstrukteure des MST die lexikalische Kombinierbarkeit russischer Lexeme erfassen, als linguistische Grundlage für die Erstellung pädagogischer Wörterbücher bezeichnet wird (APRESJAN/ZOLKOVSKIJ/MEL'CUK 1969d, 61), entstiunde unweigerlich der Eindruck, daB das Rektionsmodell und die lexikalischen Funktionen in ihren abstrakten und komplexen Notierungen für Lehr- und Lernzwecke eingesetzt werden sollen. 31

Auch wir glauben, daB die lexikalischen Funktionen des EKW von hohem lexikographischen Wert für die Beschreibung des kombinatorischen Potentials von Lexemen sind (s.u.), allerdings sollten sie in veränderter Darstellungsform in ein ein-oder zweisprachiges EKW, das sich an Nichtlinguisten wendet, aufgenommen werden. In den Wörterbuchartikeln eines EKW, das Muttersprachler befragen, um bestimmte idiomatische, gegebenenfalls lexikalisch oder stilistisch variierbare Wort- 
verbindungen nachzuschlagen, sollten weder Erläuterungen noch Symbole, sondern lediglich die Werte der lexikalischen Funktionen aufgeführt werden, die fuir das gegebene Stichwort definiert sind. Als für den Adressaten implizites Prinzip der Anordnung im Wörterbuchartikel könnte man sich dabei an der - wenn auch nicht immer eindeutig durchfiurbaren - Untergliederung der lexikalischen Funktionen in Substitutionen und Parameter sowie in bedeutungshaltige und bedeutungsleere lexikalische Funktionen orientieren. Eine explizite Darstellung der lexikalischen Funktionen ist aus der Sicht der potentiellen Benutzer des EKW nicht erforderlich, da sie als Muttersprachler die syntaktischen und semantischen Relationen, die die lexikalischen Funktionen zwischen Lexemen etablieren, bereits kennen und im Wörterbuch nur die entsprechenden idiomatischen Ausdrucksmöglichkeiten aufsuchen.

Auch für ein zweisprachiges EKW besteht unter praktischen lexikographischen Aspekten keine Notwendigkeit, die lexikalischen Funktionen in der oben dargestellten Notationsform anzugeben. Für die Bedurfnisse der potentiellen Adressaten reicht es aus, in den Wörterbuchartikeln die Werte der lexikalischen Funktionen, die in Ausgangs-und Zielsprache für ein gegebenes Stichwort definiert sind, aufzulisten. Es ist nicht erforderlich, die lexikalischen Funktionen zu explizieren, da die potentiellen Benutzer eines zweisprachigen EKW die ausgangssprachlichen Ausdrücke, die Werte lexikalischer Funktionen sind, ohnehin kennen und über diese zu den zielsprachlichen Aquivalenzen geführt werden.

Der hohe Wert, den die lexikalischen Funktionen für die lexikographische Beschreibung darstellen, liegt in ihrer sysetmatik, mit deren Hilfe sich die lexikalische Kombinierbarkeit von Lexemen in einer gegebenen Sprache erfassen läBt. Die lexikalischen Funktionen des EKW geben dem Lexikographen, der ein ein-oder zweisprachiges Wörterbuch erstellen will, ein Beschreibungsinstrumentarium an die Hand, das die Entscheidung, welche Wörter und Wortverbindungen in das Lexikon aufzunehmen sind, nicht seiner Willkür uberläßt, sondern sie von linguistisch begründeten Kriterien, 
d.h. dem jeweils angesetzten Inventar lexikalischer Funktionen, abhängig macht. Die unvollkommene und unbegründete $S y-$ stematik, mit der in Wörterbüchern das kombinatorische Potential von Lexemen erfaBt wird, bemängeln auch die Konstrukteure des MST:

"In einsprachigen (erklärenden) und zweisprachigen Wörterbüchern (Aquivalenzwörterbüchern) müssen bei den Stichwörtern alle Werte ihrer lexikalischen Funktionen angegeben werden. Bisher ist das ohne eine klare Linie geschehen: der Lexikograph strebt danach, die wichtigsten nichtfreien Wortverbindungen eines Stichworts aufzuführen, indem er sie aus Texten, Wörterbuichern u.ä. entnimmt, die er bearbeitet hat. Die Werte vieler lexikalischer Funktionen geraten dabei in die Wörterbuchartikeln (und werden dort ganz zufällig - beispielsweise alphabetisch nach ihrem ersten Wort angeordnet), viele andere Werte jedoch (und das sind offensichtlich die meisten) werden überhaupt ausgelassen." (MEL'CUK $1974 b, 107$ )

Der effektive Beitrag, den die lexikalischen Funktionen des EKW nach Ansicht der Konstrukteure des MST für bestimmte Bereiche des fremdsprachlichen (und auch muttersprachlichen) Unterrichts zu leisten vermögen, steht ebenfalls auBer Zweifel:

"Die lexikalische Kombinierbarkeit ist einer der wichtigsten Steine des AnstoBes beim Erlernen einer Fremdsprache, und zwar besonders dann, wenn ihre aktive Beherrschung angestrebt wird..." (MEL'CUK 1974b, 105)

"Im SprachlehrprozeB (nicht nur einer Fremd-, sondern auch der Muttersprache) muB bei der Wortschatzarbeit gerade dem Erlernen der lexikalischen Funktionen besondere Aufmerksamkeit gewidmet werden .... Das erlaubt es, eine konkretere und genauere Vorstellung davon zu erlangen, was es bedeutet, die Lexik der einen oder anderen Sprache zu beherrschen und die Schwierigkeiten zu bewerten, die sich dabei ergeben ... Den Wortschatz $z u$ beherrschen bedeutet nicht nur, einfach eine bestimmte Menge von Wörtern zu kennen, sondern auch für jedes Wort durchschnittlich 20 Verbindungen (die Werte der le$x i k a l i s c h e n$ Standardfunktionen und Nichtstandardfunktionen). Auf jeweils 100 Wörter ergibt das 20.000 Wortverbindungen! Es ist kein Wunder, daB der Erwerb der lexikalischen Kombinierbarkeit beim Erlernen einer Fremdsprache am schwierigsten ist. Auch in der Muttersprache ist sie nicht einfach zu erwerben - Sprecher, die gerade in diesem Bereich kundig sind, machen zahlreiche Fehler, von denen insbesondere in Zeitungen sehr viele anzutreffen sind. Aus diesem Grund wäre auch ein einsprachiges Wörterbuch, das die Werte der lexika1 ischen Funktionen angibt, ein nuitzliches Handbuch für Obersetzer, Redakteure u.a." (MEL'CUK 1974b, 107) 
Im Fremdsprachenunterricht treten in vielen Fällen immer dann Lernschwierigkeiten auf, wenn die Werte einer lexikalischen Funktion bezüglich eines gegebenen Stichworts in der Ziel-und der Ausgangssprache verschiedene idiomatische Ausdrücke haben. Es bedarf nur noch einer kontrastiven Analyse beider Sprachen, um die Werte lexikalischer Funktionen zu ermitteln, welche sich völlig oder partiell unterscheiden und daher interferenzanfällig sind. Ein weiterer Vorteil der lexikalischen Funktionen liegt darin, daB sie mit dem Anordnungsprinzip des sogenannten thematischen Wortschatzes, das oft im Fremdsprachenunterricht verwendet wird, kompatibel sind.

Die mangelhafte Systematik, mit der in Wörterbüchern die lexikalische Kombinierbarkeit von Lexemen beschrieben wird, zeigt sich ganz deutlich, wenn man beispielsweise die Wörterbuchartikel, die das EKW und das für den Unterricht des Russischen als Fremdsprache bestimmte "Učebnyj slovar" sočetaemosti slov russkogo jazyka" dem Verb učit'sja zuweisen, im Hinblick auf die Wortverbindungen, die sie auflisten, vergleicht. Im "Učebnyj slovar" sočetaemosti" finden sich etliche freie Wortverbindungen wie beispielsweise učit'sja dolgo, mnogo let, vsju žizn'; chotet', sobirat', rešit', sovetovat' komu-l., načinat', stat', prodolžat' učit'sja. Die wenigen nichtfreien Wortverbindungen, die sich im Wörterbuchartikel finden, sind sämtlich Werte der lexikalischen Funktion Magn ; vgl. uóit'sja staratel'no, userdno, uporno, nastojčivo (Učebnyj slovar' 1978, 632). Im EKW finden sich dagegen nicht nur die Werte der lexikalischen Funktion Magn, sondern darüber hinaus noch die lexikalischen Korrelate von uber zehn anderen lexikalischen Funktionen. Freie Wortverbindungen enthält der Wörterbuchartikel des EKW nicht (MEL'CUK 1974b, 128).

Zwar wendet sich das "Učebnyj slovar" sočetaemosti" gemeinhin an Nichtmuttersprachler, ohne den Wortschatz unter kontrastiven Gesichtspunkten zwischen Ziel- und Ausgangssprache und den sich daraus ergebenden Transfer- und Interferenzmöglichkeiten anzuordnen, jedoch kann das nicht seinen prinzipiellen Mangel aufwiegen, nur sehr wenige Wortverbin- 
dungen zu berücksichtigen, die im Hinblick auf bestimmte lexikalische Funktionen idiomatisch sind, auch wenn es dafür um so mehr - gelegentlich thematisch mit dem Stichwort verbundene - freie Wortverbindungen auflistet.

Wir haben - bedingt durch die Zielsetzung unserer Arbeit die lexikalischen Funktionen bisher nur unter lexikologischen und lexikographischen Aspekten besprochen. AbschlieBend sollen noch einige kurze Anmerkungen zum Status der lexikalischen Funktionen im MST gemacht werden. Die Konstrukteure des MST bezeichnen die lexikalischen Funktionen einmal als ein Beschreibungsverfahren, mit dessen Hilfe die lexikalische Kombinierbarkeit von Lexemen erfaßt werden soll (MEL'CUK 1974b, 78), andererseits erblicken sie in ihnen einen speziellen Typ von semantischen Repräsentationen (APRESJAN/ZOLKOVSKIJ/MEL'CUK 1969b, 5). Für die bedeutungshaltigen lexikalischen Funktionen, sofern sie einen verschmolzenen Ausdruck (skzeenoe vyraženie) aufweisen (s.o.), stellt sich in der Tat die Frage, ob es sich bei ihnen nicht lediglich um eine Notationsvariante für Bedeutungsexplikationen handelt und sie daher partiellen lexikalischen Dekompositionen innerhalb der semantischen Repräsentation eines gegebenen Textes entsprechen.

Ein weiteres Problem wirft die Art und Weise auf, mit der die Konstrukteure des MST bestimmte bedeutungshaltige lexikalische Funktionen bzw. deren lexikalische Korrelate spezifizieren. Die Werte der lexikalischen Funktion Magn von beispielsweise krasnyj (in der Bedeutung 'krasnyj ot moroza'), kofe und kurit' versehen sie mit den folgenden Zusätzen:

Magn (krasnyj) = kak gusinye lapy [tol'ko o rukach/nogach, krasnych ot moroza i.t.p.] (MEL'CUK $1974 \mathrm{~b}$, $104)^{31}$

Magn ['nastoj'] (kofe) = krepkij, dvojnoj [prigotoviennyj iz dvojnoj procii natural'nogo kofe $2 b$ ) (BOGDANOVA/NIKITINA/MEL'CUK $1975,5)$ 
Magn, i kurenie $x-a$

neprijatno govorjaščemu (kurit') = kak parovoz

(AL'PERIN/APRESJAN/MEL'CUK 1975, 19)

Die lexikalische Funktion Ver, deren Bedeutung die Konstrukteure des MST als 'pravil'nyj, sootvetstvujušcij naznačeniju, kakoj sleduet' angeben (MEL'CUK 1974b, 90), spezifizieren sie beispielsweise für das Stichwort vystrel durch den folgenden Zusatz:

$\operatorname{Ver}_{[\text {poražat'] }}($ vystrel $)=$ chorošij, prekrasnyj, ot ličnyj; metkij (APRESJAN/ZOLKOVSKIJ/MEL'CUK $1975,26)$

Sollte die zukünftige Forschung zeigen, daß Wortverbindungen, die mit Hilfe bestimmter bedeutungshaltiger lexikalischer Funktionen beschrieben werden können, in den meisten fällen Zusätze der oben genannten Art erfordern, so würde die Anzahl der lexikalischen Nichtstandardfunktionen die der lexikalischen Standardfunktionen übertreffen. Das Konzept der lexikalischen Funktionen könnte dann leicht ausgehöhlt werden, da den meisten ihrer Werte bzw. den lexikalischen Funktionen selost im EKW Bedeutungsexplikationen zugewiesen werden müßten.

2.2.2.3 Zum Verhältnis zwischen Bedeutung und Kombinierbarkeit der Wörter im erklärend-kombinatorischen Wörterbuch

Im Rektionsmodell, mit dessen Hilfe die kombinatorischen Eigenschaften der Lexeme im Hinblick auf ihre grammatischen Bedeutungen beschrieben werden ( $v g l$. S. $115 \mathrm{ff}$. ), unterscheiden die Konstrukteure des MST drei verschiedene Typen von Restriktionen, denen die Kombinierbarkeit der Wörter unterliegen kann. Diese drei Typen von Restriktionen betreffen 1. die morpho-syntaktische, 2. die lexikalische und 3. die semantische Kombinierbarkeit der Lexeme. Sie werden von den Konstrukteuren des MST wie folgt definiert:

"Gegeben sei ein Wort $A$, das syntaktisch direkt oder indirekt mit einem Wort (einer Wortverbindung, einem Satz) $B$ 
verknuipft ist. Die Informationen uber die Wortart oder den syntaktischen Status von $B$ und über die grammatische Form, in der $B$ stehen muB, bilden die morpho-syntaktische Kombinierbarkeit von $A$ oder die morpho-syntaktischen Restriktionen der Kombinierbarkeit von A." (APRESJAN 1974a, 323)

"Die Information uiber das Wort $B$ oder die Klasse von Wörtern $B_{1}, B_{2} \ldots B_{n}$, mit dem bzw. denen das Wort $A$ syntaktisch verknuipft werden kann, bildet die lexikalische Kombinierbarkeit von $A$ oder die lexikalischen Restriktionen der Kombinierbarkeit von $A . "$ (APRESJAN 1974a, ebd.)

"Die Information iber die semantischen Merkmale, die das Wort $B$, das mit $A$ syntaktisch verbunden ist, besitzen muB, gibt die semantische Kombinierbarkeit von $A$ oder die semantischen Restriktionen der Kombinierbarkeit von $A$ an."

(APRESJAN $1974 \mathrm{a}$, ebd.)

Die Konstrukteure des MST illustrieren die verschiedenen Typen von Restriktionen, denen die kombinatorischen Eigenschaften unterliegen können, die im Rektionsmodell eines gegebenen Stichworts beschrieben werden, u.a. anhand der folgenden Beispiele. Für die Kombinierbarkeit der Quasisynonyme soprovaždat' und soputstvovat' sowie scitat' und rassmatrivat' mit Lexemen bzw. Wortverbindungen, die ihre zweite syntaktische Valenz realisieren können, gelten verschiedene morphosyntaktische Restriktionen; vgl. die Wortverbindungen (66) (67):

(66) soprovắdat' čto-libo - soputstvovat' čemu-libo

(67) sčitat' rabotu zakončennojlčto rabota zakončena rassmatrivat' rabotu kak zakončennuju (APRESJAN 1974a, ebd.)

Lexikalischer Natur sind die Restriktionen, denen die Kombinierbarkeit der Verben ošibat'sja und sbrasyvat' mit Lexemen unterliegt, die ihre zweite syntaktische Valenz realisieren können. In beiden Fällen muissen sie einzeln aufgezählt werden, da sich in ihren lexikalischen Bedeutungen kein gemeinsames semantisches Merkmal ausmachen läßt. Das Verb ósibat'sja kann nur mit den Lexemen adres, dver', dom, nomer, okno, telefon und étaž, das Verb sbrasyvat' nur mit den Lexemen davlenie, gaz, skorost', temperatura und ves syntaktisch verknuipft werden. Die Quasisynonyme beider Verben pereputyvat' und umen'sat' unterliegen dagegen hinsichtlich ihrer Kombi- 
nierbarkeit keiner lexikalischen Beschränkung; vgl. die Wortverbindungen (68) - (69):

(68) pereputyvat' adres, telefon, kijuc, zontik

(69) umen'sat' skorost', ves, raschody, širinu (APRESJAN 1974a, ebd.)

Als Beispiele für semantische Restriktionen der Kombinierbarkeit von Lexemen führen die Konstrukteure des MST u.a. die Verben prekraščat'sja und perestavat' sowie uchudšat' sja/ulučšat'sja, stanovit'sja chuže/lučše und portit'sjal ispravljat'sja an. Bei dem Verb prekrašcat'sja können nur solche Lexeme die syntaktische Subjektvalenz realisieren, die zur semantischen Klasse der Bezeichnungen für meteorologische Niederschläge (imena osadkov) und der Bezeichnungen für Handlungen (imena dejatel'nosti) gehören, während die syntaktische Subjektvalenz bei seinem Synonym perestavat' nur durch Lexeme realisiert werden kann, die meteorologische Niederschläge bezeichnen; vgl. die Sätze (70) - (72):

(70) Dǒ̌d' prekră̌čaetsja.

(71) Zanjatija prekraščajutsja.

(72) Dožd', sneg perestaèt. (APRESJAN 1974a, ebd.)

Bei den Verben uchudšat'sja/uzučšat'sja unterliegen die Lexeme, die die Subjektstelle einnehmen können, der semantischen Restriktion, nur der Klasse der Bezeichnungen für Zustände, Fähigkeiten oder Prozesse angehörenzu dürfen. Lexeme, die konkrete Gegenstände oder Personen bezeichnen, können bei diesen Verben die syntaktische Subjektvalenz nicht realisieren. Die Kombinierbarkeit der mit den Verben synonymischen Ausdrücke stanovit'sja chuže/lučše und portit'sja/ispravijat'sja ist dagegen nicht in dieser Weise eingeschränkt; vgl. die Sätze (73) - (75):

(73) Pogoda, zrenie, povedenie uchudšaetsja/ulučs̆aetsja.

(74) Pogoda, zrenie, povedenie, Pëtr, mašina stanovitsja chuže/Zučše.

(75) Pogoda, zrenie, povedenie, Pëtr, mašina portitsja/ispravijaetsja. (APRESJAN 1974b, 61-62) 
Bei der Explikation der Bedeutung bestimmter Lexeme stellen die Konstrukteure des MST Oberlegungen darüber an, ob gewisse Bedeutungskomponenten der Wörter mit Hilfe der oben genannten Typen von Restriktionen als Eigenschaft ihrer Kombinierbarkeit oder mit Hilfe spezieller Seme als Eigenschaft ihrer lexikalischen Bedeutung im Rektionsmodell bzw. in der lexikographischen Definition beschrieben werden sollen (APRESJAN 1971a, 29; 1974b, 66ff.). Beispielsweise kann in der semantischen Beschreibung des Verbs rubit' die Bedeutungskomponente 'instrument' nur als Bestandteil seiner lexikalischen Bedeutung, d.h. als ein Sem in seiner Bedeutungsexplikation beschrieben werden. Würde man die Bedeutungskomponente in Form einer semantischen Restriktion der Kombinierbarkeit von rubit, in dessen Rektionsmodell erfassen, so könnten zwar Verwendungen des Verbs wie in (76), jedoch nicht wie in (77) beschrieben werden:

(76) Ivan rubit drova toporom.

(77) Ivan razrubil kirpič doskoj.

Für die durch rubit' bezeichnete auBersprachliche Situation und somit auch fur seine lexikalische Bedeutung ist es paradigmatisch, daB der Mitspieler, dem die Instrumentalrolle zukommt, nicht unbedingt durch Lexeme realisiert werden muB, die zur Klasse der Bezeichnungen für Instrumente wie z.B. topor gehören. Er kann auch durch solche Lexeme ausgedrückt werden, denen wie z.B. doska in der betreffenden Situation die Funktion eines Instruments verliehen wird (APRESJAN 1974b, 63).

Als ein Beispiel für Lexeme, die über semantische Eigenschaften verfugen, welche ausschlieBlich als Besonderheiten ihrer Kombinierbarkeit und nicht ihrer lexikalischen Bedeutung beschrieben werden können, führen die Konstrukteure des MST die Verben uchuď̌at'šja und ulučsat'sja an. Die semantischen Beschränkungen, denen die Kombinierbarkeit dieser Verben mit Wörtern unterworfen ist, die ihre syntaktische Subjektvalenz realisieren können (s.o.), lassen sich aus ihren lexikalischen Bedeutungen nicht herleiten, da die Kombinierbarkeit ihrer genauen Synonyme stanovit'sja chuže und stanovit'sja lučse diesen Restriktionen nicht unterliegt 
(APRESJAN 1971a, 32; 1974b, 61-62).

Die Konstrukteure des MST weisen darauf hin, daB in sehr vielen Fällen bestimmte semantische Eigenschaften von Wortschatzeinheiten als Besonderheiten von deren lexikalischen Bedeutungen oder deren kombinatorischem Potential beschrieben werden können, ohne die Vollständigkeit und Stringenz der Deskription zu beeinträchtigen:

"Tatsache ist, daB zwar nicht alle, jedoch sehr viele semantische Unterschiede mit dem gleichen MaB an Vollständigkeit und Widerspruchsfreiheit auf $z$ wei verschiedene Weisen repräsentiert werden können: 1. entweder als Unterschiede in den Bedeutungen der entsprechenden sprachlichen Einheiten oder 2. als Unterschiede in ihrer Kombinierbarkeit." (APRESJAN $1971 \mathrm{a}, 28$ )

Wörter, für deren semantische Beschreibung sich nach Ansicht der Konstrukteure des MST beide Lösungswege gleichberechtigt anbieten, sind u.a. die Lexeme svora, staja und tabun. Die drei Wörter können einerseits als Quasisynonyme beschrieben werden, indem man ihre Kombinationsrestriktionen mit $\mathrm{Hilfe}$ der Seme 'sobak', 'volkov ili ptic' und 'Zošadej' in den Explikationen ihrer Bedeutungen erfaBt und ihnen die lexikographischen Definitionen (78) - (80) zuweist:

(78) svora $=$ 'Sovokupnost' sobak'

(79) staja $=$ 'Sovokupnost' volkov ili ptic'

(80) $\frac{\text { tabun }}{\text { ( }}$ ' Sovokupnost' Lošadej' (APRESJAN 1971a, ebd.;1974b, 93).

Die oben genannten Bedeutungsexplikationen beschreiben die absolute Verwendung der Lexeme wie beispielsweise in (81):

(81) Tabun mčasija prjamo na nas.

Werden die Lexeme in Wortverbindungen wie tabun 20 sadej, staja volkcv und svora sobak verwendet, muB eine spezielle Tilgungsregel formuliert werden, die die redundanten Bedeutungskomponenten 'zošadej', 'volkov' und 'sobak' eliminiert. Andererseits ist es möglich, die drei Lexeme als genaue Synonyme mit unterschiedlichen kombinatorischen Eigenschaften $z u$ beschreiben, indem man sie als 'sovokupnost' životnyoh, nazvannuch zavisimymi suščstvitel'nymi' definiert und in ihren 
Rektionsmodellen die jeweiligen semantischen Restriktionen angibt (s.0.). Beschreitet man diesen Lösungsweg, muß man allerdings die lexikalischen Bedeutungen der drei Lexeme aufspalten und die Wörter als polysem betrachten, da in ihrer absoluten Verwendung wie in (81) eine andere Bedeutung realisiert wird (APRESJAN 1971a, 29; 1974b, 93).

Um dennoch eine begründete Entscheidung herbeiführen zu können, welche der beiden oben genannten Beschreibungen im Falle konkurrierender Lösungsmöglichkeiten vorzuziehen ist, stellen die Konstrukteure des MST zusätzliche Oberlegungen zur okonomie und Einfachheit der semantischen Beschreibung an:

"... für die Auswahl der optimalen Lösung müssen zusätzliche [d.h. neben solchen zur Vollständigkeit und Stringenz der Beschreibung (S.O.); K.H.] Erwägungen Z.B. zur Okonomie und Einfachheit hinzugezogen werden, die im übrigen in der Linguistik immer schon, wenn auch nicht in expliziter form, ausgenutzt worden sind." (APRESJAN 1971a, ebd.)

Die Konstrukteure des MST betrachten den "semantischen" Lösungsweg, d.h. die Beschreibung einer semantischen Eigenschaft eines Lexemes als Bestandteil seiner lexikalischen Bedeutung, als ökonomischer, den "kombinatorischen" Lösungsweg, d.h. die Beschreibung einer semantischen Eigenschaft eines Lexemes als Bestandteil seiner Kombinatorik, dagegen als einfacher. Die Okonomie des "semantischen" Lösungswegs ergibt sich ihrer Ansicht nach dadurch, daB die Anzahl der polysemen Wortschatzeinheiten gering gehalten werden kann, da eine Aufspaltung der lexikalischen Bedeutung des Lexems nicht erforderlich ist. Für die Einfachheit des "kombinatorischen" Lösungswegs machen sie den Umstand geltend, da $B$ keine speziellen semantischen Tilgungsregeln formuliert werden müssen:

"Die erste Beschreibung [d.h. der "semantische" Lösungsweg; K.H.J scheint ökonomischer zu sein, da sie keine Aufspaltung der Bedeutung erfordert, die zweite Beschreibung [d.h. der "kombinatorische" Lösungsweg; K.H.] scheint einfacher zu sein, da sie keine besondere Regel für die Zusammenfassung der Bedeutungen (pravilo složenija značenij) erfordert." (APRESJAN 1971a, erd.)

Die Konstrukteure des MST beabsichtigen, sich in den zahl- 
reichen Fällen von konkurrierenden Lösungsmöglichkeiten, die bei der semantischen Beschreibung von Wortschatzeinheiten auftreten können, für den "kombinatorischen" Lösungsweg zu entscheiden, da er sich durch größere Einfachheit auszeichne. Sie begrijnden ihre Entscheidung damit, daB der Wortschatz einer Sprache ein offenes System bilde und daher auch mit einer eventuell steigenden Anzahl polysemer Lexeme, die sich bei bestimmten "kombinatorischen" Lösungen ergeben könne, kompatibel sei:

"In solchen ... Fällen muß, wie die Erfahrung mit unserer Arbeit zeigt, der Einfachheit, d.h. in der Regel der kombinatorischen Lösung, der Vorrang gegeben werden ... Sie erfordert wie wir festgestellt haben, eine Aufspaltung der Bedeutungen, d.h. eine Aufspaltung des Lexikons. Eine Aufspaltung des Lexikons droht jedoch nicht mit irgendwelchen ernsten Konsequenzen, da das Lexikon seinem Wesen nach ein nichtgeschlossenes, offenes system darstellt." (APRESJAN 1971 a, ebd.)

Betrachtet man die lexikographischen Definitionen, die die Konstrukteure des MST in ihren verschiedenen Publikationen für russische Lexeme geben, so läßt sich feststellen, daß sie in etlichen Fällen das Beschreibungsprinzip vernachlässigen, bestimmte semantische Eigenschaften der Lexeme, falls möglich, als Besonderheiten von deren kombinatorischem Potential zu erfassen. In vielen Bedeutungsexplikationen des EKW finden sich Bedeutungskomponenten, die entsprechend den Absichten der Konstrukteure des MST mit Hilfe semantischer Merkmale als Kombinationsrestriktionen der betreffenden Lexeme in deren Rektionsmodellen beschrieben werden könnten. Die Konstrukteure des MST geben beispielsweise für das Verb udarjat, (in einer seiner Bedeutungen) die Explikation (82): (82) X udarjaet po $y-u$ z-om $=$ 'X rezko $i$ kratkovremenno privodit kompaktnyj predmet $2 v$ kontakt $s$ predmetom $Y . '$ (APRESJAN 1974b, 108)

Die unterstrichenen Seme in (82) können ohne weiteres in semantische Selektionsrestriktionen umgeschrieben und im Rektionsmodell von udarjat' vermerkt werden, um die Auswahl der Lexeme, die die Variablen ' $y$ ' und ' $Z$ ' realisieren können, zu steuern: 


\begin{tabular}{|l|l|l|}
\hline $1=X-S u b$ & $2=Y-o b j$ & $3=2-$ Instr. \\
\hline$s_{i m .}$ & po $\begin{array}{c}s_{\text {dat. }} \\
\text { objazat. }\end{array}$ & $s_{\text {tvor. }}$ \\
\hline
\end{tabular}

1) 2.: imena predmetov 2) 3.: imena kompaktnych predmetov

In der Bedeutungsexplikation (82) behandeln die Konstrukteure des MST jedoch semantische Eigenschaften von udarjat', deren Beschreibung für den "kombinatorischen"Lösungsweg in Frage käme, entgegen ihren ausdrücklichen Absichten als Bestandteil der lexikalischen Bedeutung des Verbs.

2.3 Zur Notwendigkeit von empirischen Untersuchungen der lexikographischen Eignung des erklärend-kombinatorischen Wörterbuchs als einsprachiges Definitionslexikon

Der Anspruch der Konstrukteure des MST, mit dem EKW eine bessere Alternative zu gängigen russischen Definitionswörterbijchern vorzulegen, ist innerhalb der sowjetischen Linguistik von mehreren Autoren kritisiert worden. Unter den Veröffentlichungen, die sich kritisch mit dem EKW auseinandersetzen, nimmt die Arbeit von KOTELOVA (KOTELOVA 1975) eine herausragende Stellung ein, da sie u.a. ausführlich auf Fragen der lexikographischen Definition im EKW eingeht. 33

Die kritischen Einwände, die seitens der sowjetischen Linguistik an Theorie und Praxis der Bedeutungsexplikation im EKW erhoben worden sind, lassen sich in vier Gruppen zusammenfassen:

1) Der SemS des EKW wird kategorisch jegliche Tauglichkeit für Zwecke der Definition von Wortbedeutungen in einem erklärenden Wörterbuch abgesprochen, weil es sich um eine künstliche Metasprache handele (KARAULOV 1976, 64-66; KISELEVSKIJ 1977, 13; KOTELOVA 1974;1975,6-46; SVEDOVA $1970,38-39)$. 
2) Die SemS des EKW wird als lexikographisches Beschreibungsinstrumentarium verworfen, weil die Konstrukteure des MST keine Auffindungsprozeduren für elementare Bedeutungen angeben können (KISELEVSKIJ 1976, 66-68, 70; KOTELOVA 1974; $1975,6-46$; SVEDOVA 1970, 43-44).

3) Der Reduktionsansatz, auf dem die Bedeutungsexplikation mit Hilfe der SemS im EKW basiert, wird abgelehnt, weil er eine intensionale Definition der sogenannten konkreten Lexik unmöglich mache (KARAULOV 1976, 64-66; KISELEVSKIJ 1973, 69; KOTELOVA 1975, 33-46; ULUCHANOV 1977, 18).

4) Dem EKW wird vorgeworfen, das Wort als eigenständige linguistische Größe weitgehend zu liquidieren, da es viele Lexeme lediglich als Bestandteil des kombinatorischen Potentials bestimmter Stichwörter beschreibt (KOTELOVA 1975, 47-103; 1977, 41; SVEDOVA 1970, 39-43).

Wir können uns mit den Argumenten der Kritiker des EKW an dieser Stelle nicht näher auseinandersetzen. Es muß allerdings betont werden, da $B$ die Kenntnisse, aufgrund derer die oben genannten Autoren den lexikologischen Ansatz im MST kritisieren, of $t$ nur unzureichend sind, so daB die entsprechenden Einwände in den meisten fällen gegenstandslos werden. Charakteristisch für die Arbeit KOTELOVA 1975 ist darüber hinaus noch, daß gelegentlich Zitate aus Veröffentlichungen der Konstrukteure des MST aus dem Zusammenhang gerissen und entstellt wiedergegeben werden.

Die Diskussion, die in der Sowjetunion um den lexikologischen Ansatz der Konstrukteure des MST und insbesondere um die SemS des EKW geführt wurde, nimmt im übrigen nicht nur in theoretischen linguistischen Veröffentlichungen einen breiten Raum ein; sie hat auch bereits einen Niederschlag in der russischen Lexikographie gefunden. Im Vorwort zur 10. Auflage des "Slovar' russkogo jazyka" von OZEGOV (OZEGOV 1973) wird die Sprache, mit deren Hilfe Wortbedeutungen im Wörterbuch definiert werden, ausdrücklich als das moderne Russische bezeichnet und die Forderung, eine künstliche Metasprache als Definitionssprache zu verwenden, wird abgelehnt: 
"Die Sprache der Bedeutungsexplikation ist die russische Sprache der Gegenwart geblieben und nicht etwa eine speziell konstruierte 'semantische Sprache'." (OZEGOV 1973, 8)

Dem pauschalen Einwand der einschlägigen sowjetischen Kritik, daß die Sems für praktische lexikographische Zwecke ungeeignet sei und daher das EKW keine bessere Alternative zu gängigen russischen Definitionswörterbuchern biete, steht die Behauptung der Konstrukteure des MST gegenüber, daB das EKW gerade deswegen anderen Definitionswörterbuchern uberlegen sei, weil es sich bei der Bedeutungsexplikation einer küntlichen Definitionssprache bediene, die die Normen der russischen Stilistik und Grammatik - allerdings in nur sehr geringem Maße; vgl.S. 61 - verletze:

"... der metasprachliche Text der Definition soll und kann im allgemeinen kein idiomatisch russischer Text sein ... Charakteristisch für viele gute Definitionen in erklärenden Wörterbuichern ist es, daB sie von stilistischen, lexikalischen und sogar syntaktischen Normen der gegebenen Sprache abweichen. Definitionen, in denen der Lexikograph nicht als Wissenschaftler, sondern als Muttersprachler seinem naturlichen Wunsch nachgibt, sich idiomatisch auszudrücken, enthalten oft Ungenauigkeiten." (APRESJAN 1974b, 96)

Angesichts der Tatsache, daB die Auseinandersetzung um die lexikographische Eignung des EKW als einsprachiges Definitionswörterbuch bisher rein theoretisch geführt worden ist, wird die Notwendigkeit, empirische Untersuchungen zu dieser Frage vorzunehmen, immer dringlicher. Ein mögliches empirisches Verfahren wollen wir im folgenden vorstellen und durchfüren. 


\section{Festlegung des Testverfahrens ${ }^{34}$}

Der Versuch, mit Hilfe eines empirischen Verfahrens die Frage einer Beantwortung näher zu bringen, ob die Sems des EKW für lexikographische Definitionen in einem Wörterbuch, das sich an einen bestimmten noch zu spezifizierenden Kreis von Benutzern wendet ( $\mathrm{vgl}$. S. $146 \mathrm{ff}$.) geeignet ist, macht es erforderlich, Kriterien festzulegen, hinsichtlich derer wir unsere Aussagen treffen können. Für unsere Zwecke wählen wir als Kriterium die Verstehbarkeit der SemS des EKW (s.u.). Unsere Entscheidung zugunsten dieses Kriteriums gründet sich auf ein Postulat, das von MEL'CUK im Zusammenhang mit der SemS des EKW erhoben wird. Ober die lexikographische Definition im EKW schreibt er:

"Die Explikation richtet sich an den Menschen [im Gegensatz zu einer Explikation für Automaten; K.H.] ... Von der Explikation muB hinreichende sprachliche Glätte (jazykovaja gladkost') und Lesbarkeit (citabel'nost') erwartet werden." (MEL'CUK 1974b, 111)

MEL'CUKs Postulat an die Bedeutungsexplikationen des EKW zielt auf die für jedes Definitionswörterbuch selbstverständliche Tatsache $a b$, daß Wortbedeutungen nur dann hinreichend gut expliziert werden können, wenn die verwendete Definitionssprache von den Wörterbuchbenutzern auch verstanden wird.

Die Verstehbarkeit von Sprache allgemein ist unserer unmittelbaren Beobachtung nicht zugänglich, da sie das Ergebnis von psychischen Vorgängen ist, die im Gehirn eines Sprechers bei der Dekodierung ablaufen. Wir sind daher vor die Notwendigkeit gestellt, ein operationales Verfahren zu finden, das es uns erlaubt, Aussagen über die Verstehbarkeit der Sems des EKW zu machen. Zuvor soll jedoch präzisiert werden, welche Eigenschaften der Definitionssprache des EKW wir unter den Begriff ihrer Verstehbarkeit subsumieren.

Die Verstehbarkeit der SemS des EKW betrachten wir im folgenden stets im Hinblick auf konkrete Bedeutungsexplikationen, in denen diese verwendet wird. M.a.W., wir begreifen die Verstehbarkeit der Sems nicht als eine absolute Eigenschaft, 
die sämtlichen lexikographischen Definitionen eigen ist, die sich ihrer bedienen. Wenn wir von der Verstehbarkeit der SemS des EKW sprechen, haben wir vielmehr eine relationale Eigenschaft im Sinn, da wir immer im Bezug auf eine gegebene Bedeutungsexplikation als der Explikation der Bedeutung eines ganz bestimmten Stichworts fragen, ob diese für die noch zu spezifizierenden potentiellen Benutzer des EKW verständich ist oder nicht. Sprechen wir im folgenden der Kürze halber von der Verstehbarkeit der Sems, so muB dabei stets berücksichtigt werden, daB wir die im oben präzisierten Sinne relationale, d.h. auf ganz bestimmte konkrete Bedeutungsexplikationen bezogene Verstehbarkeit der semantischen Metasprache des EKW meinen.

Zwei Entdeckungsprozeduren scheinen für unsere Zwecke denkbar. Das eine heuristische Verfahren besteht darin, daB wir die in der Sems abgefaßten Bedeutungsexplikationen, die Gegenstand unseres Versuchs sind, Versuchspersonen in Form $e i-$ ner Instruktion vorlegen. Diese soll die Informanten anweisen, eine bestimmte nichtsprachliche AnschluBhandlung zu vollziehen, die wir direkt beobachten und hinsichtlich ihrer Entsprechung mit der in der Sems kodierten Instruktion beurteilen können.

Wir wollen dieses Verfahren an einem Beispiel verdeutlichen. APRESJAN schlägt für das Verb udarjat' (in einer seiner Bedeutungen) die lexikographische Definition (1) vor:

(1) X udarjaet po $Y-u$ Z-om $=$ 'X rezko $i$ kratkovremenno privodit kompaktnyj predmet $y$ v kontakt 8 predmetom 2.'

(APRESJAN 1974b, 108)

Wir besetzen nun die Stellen der Variablen ' $Y$ ' und ' $Z$ ' mit Wortformen der Lexeme stol und ukazka, führen die aufgrund von Semredundanz erforderlichen Veränderungen an der Bedeutungsexplikation durch (vgl. S. $179 \mathrm{f}$.) und schreiben sie in die Instruktion ( $\left.1^{\prime}\right)$ um, die wir Versuchspersonen vorlegen:

(1') Privodite ukazku rezko i kratkovremenno v kontakt 80 stolom! 
Von der Verstehbarkeit der Bedeutungsexplikation (1) wollen wir dann sprechen, wenn Versuchspersonen durch die Anweisung ( $l^{\prime}$ ) veranlaBt werden, die in ihr kodierte AnschluBhandlung durchzuführen, wozu ihnen die durch stol und ukaz$k a$ bezeichneten Realien beim Test selbstverständlich zur Verfügung stehen.

Die Tauglichkeit der oben genannten behavioristisch orientierten Entdeckungsprozedur muB jedoch fur unser Ziel durch zwei Vorbehalte eingeschränkt werden. Der eine Vorbehalt ist prinzipieller, der andere technischer Natur.

Der prinzipielle Einwand gegen das Verfahren besteht darin, daB Versuchspersonen nur dann in der Lage sind, nach erfolgreicher Dekodierung eine nichtsprachliche, d.h. eine beobachtbare und somit hinsichtlich der Verstehbarkeit der entsprechenden Bedeutungsexplikation beurteilbare AnschluBhandlung zu vollziehen. wenn ihnen in Instruktionsform die Bedeutungsexplikationen von Verben vorgelegt werden, die auch wirklich solche Handlungen bezeichnen. Die Bedeutungen einer großen Menge von Verben sind daher für diese Entdeckungsprozedur nicht zugänglich oder, m.a.W., das vorgeschlagene Testverfahren ist für die klasse der Verben nicht valide. AuBerdem ist es unmöglich, dieses Testverfahren auf Bedeutungsexplikationen von Lexemen anzuwenden, die anderen Wortklassen angehören.

Die technische Schwierigkeit, die die Anwendung des oben genannten heuristischen Verfahrens beeinträchtigt, ergibt sich aus dem Stand der konkreten lexikographischen Beschreibung des Russischen im MST. In den verschiedenen Publikationen der Konstrukteure des MST werden für das EKW Zwar hauptsächlich Verben expliziert, die Bedeutungen der Mehrzahl der beschriebenen Verben lassen sich jedoch aus den oben genanten Gründen mit Hilfe dieses Verfahrens nicht erfassen.

Wegen dieser Schwierigkeiten entscheiden wir uns für eine andere Entdeckungsprozedur. Sie besteht darin, daB wir Versuchspersonen ähnlich wie beim oben genannten heuristischen Verfahren die Aufgabe stellen, gemäB einer vorgegebenen Testanweisung bestimmte AnschluBhandlungen zu vollziehen, die je- 
doch sprachlicher Natur sein sollen. Wir bezeichnen diese sprachlichen AnschluBhandlungen als Reduktionsparaphrasierungen.

Unter einer Reduktionsparaphrasierung verstehen wir eine sprachliche Operation, bei der Versuchspersonen einen vorgegebenen Satz in einen anderen Satz umformen sollen, der zu dem Ausgangssatz in Synonymierelation steht, sich aber von diesem dadurch unterscheidet, daB in ihm bestimmte, besonders gekennzeichnete Lexeme des Ausgangssatzes auf ein einziges Lexem reduziert worden sind (vgl. S. $221 \mathrm{ff}$. ). Wir wollen die Operation der Reduktionsparaphrasierung an einem Beispiel illustrieren. Indem wir die Stellen der Variablen in der Bedeutungsexplikation (2), die APRESJAN fïr das Verb udaljat'sja (in einer seiner Bedeutungen) gibt, durch Wortformen der Lexeme Lodka und bereg besetzen, erhalten wir ( $\left.2^{\prime}\right)$ als Ausgangssatz fir die Paraphrasierung:

(2) A udaljaetsja ot $B-a=$ 'A peremeščaetsja, i rasstojanie ot $A-a$ do $B-a$ uveličivaetsja.' (APRESJAN 1974b, 108)

(2') Lodka peremeščaetsja, i rasstojanie ot lodki do berega uveličivaetsja.

Weisen wir eine Versuchsperson an, (2') in einen synonymischen Satz umzuformen, wobei die in ( $\left.2^{\prime}\right)$ nicht unterstrichenen Lexeme hinsichtlich ihrer syntaktischen Funktion invariant bleiben sollen, während die unterstrichenen Lexeme auf ein einziges Lexem zu reduzieren sind, das der Wortklasse der Verben angehört, so ergibt sich (im Idealfall, vgl. S. 232) die Reduktionsparaphrase (2"): 35

(2") Lodka udaljaetsja ot berega. 36

Das Testverfahren der Reduktionsparaphrasierung, d.h. der sprachlichen AnschluBhandlungen, bietet gegenuber dem oben genannten Verfahren der nichtsprachlichen AnschluBhandiungen den Vorteil, auf Bedeutungsexplikationen von Lexemen sämtlicher Wortklassen anwendbar zu sein.

Bedienen wir uns des heuristischen Verfahrens der Reduktionsparaphrasierung, so treffen wir also unsere Aussagen über die 
Verstehbarkeit der SemS des EKW hinsichtlich der Bedeutungsrelationen, die zwischen einem ganz bestimmten Satz mit dem Stichwort, dessen lexikographische Definition wir in unseren Versuch einbringen, und den Reduktionsparaphrasen dieser lexikographischen Definition bestehen. Stehen die Reduktionsparaphrasen, in die unsere Informanten die jeweils zu testende lexikographische Definition umformen, zu dem Satz, der ausschlieblich das gesuchte Stichwort und diejenigen Wörter enthalten darf, durch die die in der zu paraphrasierenden Bedeutungsexplikation vorhandenen Variablen besetzt worden sind, in Synonymierelation, so wollen wir im Hinblick auf diese Bedeutungsexplikation von der Verstehbarkeit der Sems des EKW sprechen (vgl. S. $141 \mathrm{f}$.). Wir gehen dabei ganz einfach davon aus, daB unser Test valide ist, d.h., daB mit Hilfe des von uns verwendeten Testverfahrens auch wirklich das getestet werden kann, worum es uns zu tun ist - die Eignung der SemS des EKW für praktische lexikographische Zwecke. 


\section{AUSWAHL DER INFORMANTEN}

Bei der Verstehbarkeit der SemS des EKW handelt es sich zugegebenermaßen um ein schwer kontrollierbares Kriterium, da diesbezügliche Aussagen nur im Hinblick auf den Kreis von Sprechern gemacht werden können, für die das Wörterbuch mit seinen Bedeutungsexplikationen bestimmt sein soll.

Aus diesem Grund durfen wir unseren Test nicht bedenkenlos mit jedem beliebigen Sprecher durchführen, der das Russische als Muttersprache beherrscht. Bekanntermaßen verfügen Sprecher einer Sprachgemeinschaft uber ihre Muttersprache in sehr unterschiedlicher Weise. Um zu vermeiden, daB die Verstehbarkeit der sems des EKW, die wir in unserem Versuch ermitteln wollen, einer so hohen Varianz unterliegt, daB kaum noch verläßliche Aussagen uber deren lexikographische Verwendbarkeit gemacht werden können, mijssen wir zunächst Auswahlkriterien aufstellen und begründen, denen unsere Informanten genügen müssen.

Leider findet sich in keiner der Publikationen der Konstrukteure des MST eine Spezifizierung der Adressatengruppe des EKW. Dieser Mangel, den nicht nur das EKW, sondern auch andere Wörterbicher aufweisen, ist um so schwerwiegender, als die Frage nach der Zielgruppe für den Aufbau eines Wörterbuchs von besonderer Wichtigkeit ist. Um eine ungefähre Vorstellung uber den Kreis von Sprechern zu gewinnen, die die Konstrukteure des MST als potentielle Benutzer des EKW ansehen, müssen wir uns mit den durftigen Hinweisen begnügen, die MEL'CUK in zwei seiner Arbeiten zum Verwendungszweck des Wörterbuchs gibt.

MEL'CUk stellt fest, daB sich Verstöße gegen die lexikalischen Normen der russichen Standardsprache sogar bei gebildeten Sprechern des Russischen (obrazovannye nositeli jazyka) beobachten lassen. Als Beleg fuihrt er folgende Beispiele aus Zeitungstexten an:

(3) "Polnym chodom rassirirjaetsja izbiratel'naja kampanija. (APRESJAN/ŽOLKOVSKIJ/MEL'CUK 1969d, 61) 
(4) "Naše otnošenie na rol' pečati (MEL'CUK 1974b, 132)

(5) "Nesbytočnye potugi (MEL'CUK 1974b, ebd.)

Die Abweichungen in diesen Sätzen bzw. Wortverbindungen erblickt MEL'CUK in Verstößen gegen den Bedeutungsumfang (3) bzw. die Kombinierbarkeit (4), (5) der unterstrichenen Lexeme. Mit Hilfe des EKW hätten seiner Meinung nach derartige Fehler vermieden werden können, da dieses seinen Benutzern sämtliche Informationen zur Verfügung stelle, die zur Bildung der im Vergleich zu (3) - (5) wohlgeformten Sätze bzw. Wortverbindungen $\left(3^{\prime}\right)-\left(5^{\prime}\right)$ erforderlich seien:

(3') Polnym chodom razvërtyvaetsja/rasprostranjaetsja izbiratel'naja kampanija.

(4') Nắe otnošenie k roli pečati

(5') Tšcetnye potugi

Sollen die potentiellen Benutzer des EKW zu derjenigen Gruppe von Sprechern gehören, die ein Lexikon befragen, um sich uber den Bedeutungsumfang bzw. die Kombinierbarkeit bestimmter Lexeme $z u$ informieren, so halten wir die Annahme für durchaus plausibel, daB diese potentiellen Adressaten des EKW uber ein gewisses Maß an Sprachreflexion bezüglich ihrer Muttersprache verfügen.

Unter dem Begriff der Sprachreflexion wollen wir die Neigung und die Fähigkeit von Sprechern verstehen, in Fällen, in denendie Intuition über die eigene Muttersprache sie verläßt, normative Grammatiken bzw. Wörterbücher zu benutzen und die muttersprachlichen Strukturbeschreibungen dieser Nachschlagewerke zu verstehen. Mit unserer Definition des Begriffs der Sprachreflexion haben wir versucht, aus MEL'CUKs Aussagen über die Verwendungszwecke des EKW, die implizit auch Eigenschaften der Adressatengruppe des EKW beinhalten, diejenigen Charakteristika der potentiellen Benutzer des Lexikons abzuleiten und zu explizieren, welche er unter den wenig präzisen Begriff des gebildeten Sprechers des Russischen (obrazovannyj nositel' jazyka) subsumiert.

Um das Kriterium der Verstehbarkeit der Sems einigermaßen steuerbar zu machen, müssen wir diese daher hinsichtlich der Adressatengruppe des EKW untersuchen. M.a.W., für un- 
seren Test dirfen wir uns nur auf solche Sprecher des Russischen stuitzen, die über ein gewisses MaB an Sprachrefle$x$ ion im oben genannten Sinn verfügen. Uns stehen keine empirischen Verfahren zur Verfügung, mit deren Hilfe wir die unterschiedlichen Grade von Sprachreflexion, die verschiedenen Sprechern einer Sprache zueigen sein können, ermitteln können. Um dennoch unseren Versuch mit Sprechern des Russischen durchführen zu können, die uber ein auch nur annähernd gleiches Maß an Sprachreflexion verfügen, sind wir gezwungen, weitere Festlegungen bei der Auswahl unserer Versuchspersonen zu treffen. Diese beziehen sich auf zwei unmittelbar beobachtbare Eigenschaften von Sprechern, nämlich das Alter und die Schulbildung. In Ermangelung geeigneter Kriterien korrelieren wir Alter und Schulbildung der Versuchspersonen mit der Ausprägung an Sprachreflexion, über die sie verfügen. Um zu erreichen, daB die Gruppe unserer Informanten bezüglich dieser fähigkeit auch nur annäherungsweise homogen ist, legen wir fest, daB für unseren Test nur solche Sprecher des Russischen herangezogen werden können, die erwachsen sind und die sowjetische zehnklassige polytechnische Schule (Srednjaja škola) abgeschlossen haben. Trotz aller Vorbehalte, die im Zusammenhang mit diesen Auswahlkriterien für unsere Versuchsperson geltend gemacht werden müssen, sehen wir unsere Entscheidung zugunsten von Alter und Schulbildung durch empirische Untersuchungen zur Semantik und Syntax, die andere Linguisten wie be ispielsweise FAUST (FAUST 1978, 382), KARAULOV (KARAULOV 1376, 96) und KOPYLENKO (KOPYLENKO 1973, 37) vorgelegt haben, zumindest aufgrund der linguistischen Beschreibungspraxis abgesichert. In den genannten Arbeiten wird ebenfalls versucht, mit Hilfe ähnlicher Anforderungen an Alter und Schulbildung Homogenität der Informanten bezuglich ihrer Sprachreflexion zu erzielen. 


\section{KONSTRUKTION DES TESTS}

Wenn es das Ziel unserer Bemuihungen sein soll, die Tauglichkeit der SemS des EKW für lexikographische Zwecke zu ermitteln, so durfen wir unser Testverfahren nicht ausschlieblich auf diese beschränken. Praktische lexikographische Bedurfnisse wollen auch andere einsprachige erklärende Wörterbücher des Russischen befriedigen, welche eine Definitionssprache verwenden, die sich von der SemS des EKW erheblich unterscheidet. Es ist daher notwendig, die Definitionssprache zumindest eines dieser Wörterbücher sozusagen als Vergleichsinstanz in unsere Betrachtung miteinzubeziehen. Diese muB ebenfalls unserem Test unterzogen und hinsichtlich ihrer Verstehbarkeit untersucht werden. Wir führen deshalb unseren Versuch auch mit der Definitionssprache des Wörterbuchs von OZEGOV (OZEGOV 1973) durch. Unsere Entscheidung zugunsten des Lexikons von OZEGOV gründet sich auf die umfassende Kritik, die die Konstrukteure des MST an Konzeption und Beschreibungspraxis dieses gängigen russischen Definitionswörterbuchs äuBern (vgl. beispielsweise APRESJAN 1969a). Erst wenn wir über die Ergebnisse beider Tests verfügen, können wir Aussagen uber die praktische lexikographische Verwendbarkeit der SemS des EKW machen. Wir gelangen zu unserer Beurteilung der SemS des EKW, indem wir die Resultate der beiden Versuchsreihen einander gegenüberstellen. Die Verwendbarkeit bzw. Untauglichkeit der SemS des EKW für lexikographische Zwecke wollen wir daher auch nicht als eine ihr innewohnende absolute Eigenschaft auffassen. Wir bewerten ihre Eignung fur die einsprachige Lexikographie des Russischen vielmehr relativ zur Definitionssprache des Wörterbuchs von OZ̈EGOV. 
5.1 Probleme der Ver.gleichbarkeit des erklärend-kombinatorischen Wörterbuchs im Modell "Smys $2 \rightarrow$ Tekst" und des Definitionswörterbuchs von OZEGOV

Beziehen wir die Definitionssprache des Wörterbuchs von OZEGOV in unseren Test mit ein, stellt sich natiurlich die Frage, ob beide Wörterbuicher uberhaupt vergleichbar sind. Wichtig für unser Vorhaben ist es, daß die Vergleichbarkeit beider Lexika unter den folgenden drei Gesichtspunkten gewährleistet ist:

a) Ist es zulässig, die Definitionssprache des Wörterbuchs von OZ̈EGOV dem gleichen Testverfahren wie die Sems des EKW zu unterziehen, obwohl wir es bei der ersteren mit einer Variante der natiurlichen Sprache, bei letzterer aber mit einer Kunstsprache zu tun haben?

b) Ist die Adressatengruppe, an die sich das Wörterbuch von OŽEGV wendet, vorausgesetzt, sie wird überhaupt definiert, mit dem Kreis der potentiellen Benutzer, den wir für das EKW rekonstruiert haben, vergleichbar? Dürfen wir also unsere Informanten, mit denen wir den Versuch durchfühen wollen, der die Definitionssprache des Wörterbuchs von OZEGOV zum Gegenstand hat, nach den gleichen Homogenitätskriterien aussuchen, die wir bei der Auswahl der Versuchspersonen für den Test anlegen, der auf die sems abzielt?

c) Können die Bedeutungsexplikationen, die wir dem EKW und dem Wörterbuch von OZZEGOV für unsere Versuche entnehmen, hinsichtlich ihres Aufbaus und Umfangs miteinander verglichen werden?

$\mathrm{Zu}$ a)

Zielsetzung unseres Tests ist es, Aussagen über die lexikographische Verwendbarkeit der SemS des EKW zu machen. Die Definitionssprache sowohl des EKW als auch des Wörterbuchs von OZZEGOV erheben gleichermaßen den Anspruch, praktische lexikographische Bedurfnisse $z u$ erfillen, genauer gesagt, ein geeignetes Instrumentarium für lexikographische Definitionen zu sein. Mit unserem Versuch wollen wir ausschlieb- 
lich diese Zweckbestimmung der Definitionssprachen beider Lexika uberprüfen. Die Fragestellung und das Testverfahren, die unserem Versuch zugrundeliegen, werden daher von der Tatsache, daß es sich um zwei Definitionssprachen mit unterschiedlichen strukturellen Eigenschaften handelt, in keiner Weise berüht.

Zu b)

Die Adressatengruppe, an die sich die Ausgabe des Wörterbuchs von OZEGOV wendet, die wir für unseren Test heranziehen wollen, wird von ihren Herausgebern in gleich unzureichender Weise charakterisiert, wie es auch die konstrukteure des MST für das EKW tun. Im Redaktionsvorwort zur 10. Ausgabe des Lexikons findet sich nur die lapidare Feststellung, dab es sich um ein

"... normatives, allgemeinverständliches Handbuch Ihandelt; K.H.l, das dazu bestimmt ist, einen Bejtrag zur Erhöhung der Sprachkultur der breiten Volksmassen (sirokie narodnye massy) zu leisten, und eine Anleitung zur richtigen Verwendung der Wörter. Formbildung, Aussprache und Schreibung darstellt." (OZEGOV 1973, 5)

Wir können getrost die Homogenitätskriterien, denen die Informanten genügen müssen, mit denen wir die SemS des EKW testen wollen, auch für die Auswahl der Versuchspersonen zugrundelegen, mit deren Hilfe wir die Definitionssprache des Wörterbuchs von OZZEGOV untersuchen wollen. Dabei ist es letztlich belanglos, ob unsere Anforderungen an Alter und Schulbildung der Versuchspersonen, die wir erheben, um annähernde Homogenität hinsichtlich ihrer Sprachreflexion zu erzielen, noch den kreis von Sprechern beschreibt, der unter den Begriff der breiten Volksmassen fällt, so wie ihn die Redaktion des Wörterbuchs von OZ̈EGOV versteht. Auch wenn die Gruppe unserer Informanten bezüglich der potentiellen Benutzer des Wörterbuchs von OZEGOV uberbestimmt sein sollte, wird die Verstehbarkeit seiner Definitionssprache, aufgrund derer wir unsere Aussagen über ihre lexikographische Verwendbarkeit machen wollen, zumindest nicht negativ beeinfluBt. 
5.1.1 Zur Vergleichbarkeit der Dekompositionsgrade konkurrierender lexikographischer Definitionen

$\mathrm{Zu}$ c)

Vergleichen wir die lexikographischen Definitionen des EKW mit denen des Wörterbuchs von OZEGOV, stellen wir auf Anhieb erhebliche strukturelle Unterschiede fest. Die Wörterbuchartikel von OZEGOV dekomponieren die Bedeutung des zu explizierenden Stichworts in weit geringerem Maße in Seme als die des EKW. M.a.W., die Bedeutungsexplikationen des EKW sind gegenüber denen des Wörterbuchs von OZ̈EGOV informativer, da sie mehr Angaben über die Struktur der zu explizierenden Lexembedeutung machen.

Um die unterschiedlichen Dekompositionsgrade zu illustrieren, mit der beide Lexika Lexembedeutungen beschreiben, greifen wir aus den zahlreichen Beispielen die lexikographischen Definitionen heraus, die das wörterbuch von OZEGOV (6) und das EKW (7) für eine der Bedeutungen des Stichworts učit'sja geben:

(6) $\underline{\text { ucit'sja }}$ = 'Usvaivat' kakie-n. znanija, navyki' (OZEGOV 1973,777$)^{37}$

(7) $X$ učitsja $Y-u=' x$, soznatel'no zatračivaja usilija, stremitsja priobresti znanija ili umenija $Y . '$

(MEL'CUK 1974b, 127)

Der niedrige Dekompositionsgrad, durch den sich die Bedeutungsexplikationen des Wörterbuchs von OZEGOV im Vergleich zu denen des EKW auszeichnen, ist von den Verfassern des Lexikons beabsichtigt. Im Vorwort des Wörterbuchs weist SVEDOVA darauf hin, daB sich die Definitionspraxis des Lexikons an den bereits von OZZEGOV aufgestellten lexikologischen Prinzipien der Kürze (kratkost') und der Verallgemeinerung (obobščënnost") orientieren. Diese Prinzipien reglementieren den Text der Bedeutungsexplikation so, daß ein, wie SVEDOVA ihn nennt,

"... dem Wörterbuch eigener einfacher und lakonischer Stil ..." (OZEGOV 1973,7)

der lexikographischen Definition entsteht. 
Man muB OZZEGOV selbst zu Worte kommen lassen, um in Erfahrung zu bringen, was er unter Kürze und Verallgemeinerung der Bedeutungsexplikation versteht. AufschluBreich in diesem Zusammenhang ist die von OZ̈EGOV selbst verfaßte und von den Herausgebern der 10. Auflage des Wörterbuchs nur unwesentlich veränderte Anleitung zur Benutzung des Lexikons. Unter dem Punkt der Anleitung "Die Explikation der Wortbedeutung" (OŽEGOV 1973, 11-12) beschreibt OZZEGOV die Eigenschaften seiner lexikographischen Definitionen:

"\$ 11. Im Wörterbuch wird die Wortbedeutung in einer $k u r z$ e $n$ Definition erläutert, die für das Verständnis des Wortes selbst und fur seine Verwendung im $h$ e $u t i$ $g$ e $n$ Sprachgebrauch ausreicht. Es versteht sich daher, daB man vom Wörterbuch keine Angaben fordern darf, die mit sämtlichen Aspekten des [durch das Stichwort bezeichneten; K.H.] Gegenstands selbst vertraut machen.

\$ 12. Die kurzen Definitionen für Wörter mit einer oder mit mehreren Bedeutungen erfassen nur diejenigen Bedeutungen der Wörter .... die in der standardsprache stabil sind und im $h$ e $u t i g$ e $n$ Sprachgebrauch vorkommen...

$\S 14$. Im allgemeinen wird die Explikation der Wörter in möglichst kurzer Form gegeben, da die Wörter, die in der Explikation stehen, ihrerseits erklärt sind und leicht aufgesucht werden können." (OZEGOV 1973, 11-12)

Aus OZEGOVs Worten wird deutlich, daß die Verallgemeinerung der lexikographischen Definition Idealisierungen des Beschreibungsgegenstands bezeichnen, die in der Regel in semantischen Deskriptionen wie z.B. auch dem MST gemacht werden (vgl. S. $111 \mathrm{ff}$ ). Hinter dem Prinzip der Küze der lexikographischen Definition verbirgt sich ein auf Beschreibungsökonomie abzielendes Explikationsverfahren, das auch im EKW Anwendung findet und dort als das erweiterte Wörterbuch bezeichnet wird (vgl. S. $20 \mathrm{ff}$.).

Es scheint daher möglich, die Bedeutungsexplikationen von OZZEGOV mit denen des EKW vergleichbar zu machen. Da nach OZZEGOVs Worten alle Wörter, die im Definiens einer lexikographischen Definition vorkommen, ihrerseits wieder im Wörterbuch expliziert werden, können wir diejenigen Bedeutungsexplikationen, die wir für unsere Zwecke dem Lexikon entnehmen, wieder expandieren, indem wir bestimmte Wörter des De- 
finiens durch ihre lexikographische Definition ersetzen (vgl. S. $170 \mathrm{ff}$ ). Wenn wir die lexikographischen Definitionen auf diese Weise entfalten, um sie mit den Bedeutungsexplikationen des EKW bezïglich ihres Dekompositionsgrades vergleichen zu können, so sind wir uns darüber im klaren, daB wir nur eine annähernde Vergleichbarkeit zwischen beiden herbeiführen. Hierin düfte auch ein potentieller Störfaktor liegen, der die Aussagekraft unseres Versuches schmälern könnte. Die Verstehbarkeit der SemS des EKW und der Definitionssprache des Wörterbuchs von OZEGOV, bezüglich derer wir inre jeweilige Verwendbarkeit für lexikographische Bedurfnisse beurteilen wollen, hängt u.a. von dem Grad ab, in dem eine gegebene Explikation die Bedeutung des Stichworts dekomponiert. Angesichts des heutigen Forschungsstandes der linguistischen Semantik bleibt uns nichts anderes ubrig, als diese potentielle Störquelle bei unserem Test in Kauf zu nehmen. Es wäre natürlich für unser Testverfahren ideal, mit intersubjektiven kriterien operieren zu können, um von zwei gegebenen lexikographischen Definitionen desselben Stichworts zu entscheiden, ob sie vergleichbare Dekompositionsgrade aufweisen. Solche kriterien stehen uns jedoch nicht zur Verfügung.

Im folgenden wollen wir die Problematik näher besprechen, die sich bei dem Versuch ergibt, derartige Kriterien festzulegen. Wir verfolgen mit unserer Diskussion das Ziel, anhand einer konkreten Fragestellung, die die Konstruktion unseres Tests aufwirft, zu demonstrieren, welche Schwierigkeiten entstehen, wenn konkurrierende semantische Beschreibungen auf intersubjektiv nachvollziehbare Weise bezuglich einer Eigenschaft wie beispielsweise ihres relativen Dekompositionsgrades bewertet werden sollen.

Dazu gehen wir von einer quantitativen Oberlegung aus. Diese besteht darin, daB wir den Dekompositionsgrad zweier gegebener lexikographischer Definitionen desselben Stichworts durch die Anzahl der Seme bestimmen, die in ihnen verwendet werden. In diesem Zusammenhang mussen wir entscheiden, ob zur Menge der Seme auch die Kasusrollen bzw. deren konkrete lexikalische Realisierungen, die in der Bedeutungsexplikation eines Stich- 
worts angesetzt werden, zu zählen sind oder nicht. Wir schlieBen uns der Auffassung APRESJANs an, der im Gegensatz zu den Bedeutungen der Lexeme, die die vorgesehenen Leerstellen für Kasusrollen auffüllen können, die Kasusrollen als solche als Komponenten der Bedeutung des Stichworts ansieht (APRESJAN 1974b, 142). In Bedeutungsexplikationen, die wie im Wörterbuch von OZEGOV Kasusrollen nicht uber Variable ausweisen, sondern konkrete lexikalische Besetzungen verwenden, können wir daher die jeweiligen Auffüllungen dieser Leerstellen als eine Möglichkeit des Ausdrucks der Kasusrollen oder - in der Terminologie APRESJANs - der semantischen Valenzen auffassen.

Oberpruifen wir die Vergleichbarkeit von Bedeutungsexplikationen desselben Stichworts im Hinblick auf ihren Dekompositionsgrad auf diese Weise, so müssen wir z.B. den lexikographischen Definitionen, die im EKW (8) und bei entsprechender Expandierung im Wörterbuch von OZ̈EGOV (9) fïr eine der Bedeutungen des Verbs udarjat, gegeben werden, sehr ähnliche Dekompositionsgrade und somit ein sehr hohes Maß an Vergleichbarkeit zuweisen, da sie aus 10 (EKW) bzw. 9 (OZEGOV) Bedeutungskomponenten bestehen (vgl. zur Expandierung der lexikographischen Definitionen des Wörterbuchs von OZEGOV S. $170 \mathrm{ff.}$ ):

(8) Učitel' udarjaet ukazkoj po stolu. =

'Ucitel' rezko i kratkovremenno privodit ukazku v kontakt so stolom.' (APRESJAN 1974b, 108)

$\underline{\text { udarjat' }}=$ 'Proizvodit' udar obo čto-n.'

(OŽEGOV 1973,758)

$\underline{u d a r}=$ 'Rezkij, sil'nyj tolčok, prikosnovenie k komu-n.l čemu-n. 8 siloj' (OŽEGOV 1973, ebd.)

(9) Učitel' udarjaet po stolu. = 'Proizvodja rezkij tolčok, ucitel' prikasaetsja s silojkstolu.'

Zwar kann man das Abzählen der Seme als solches in den beiden oben genannten Bedeutungsexplikationen sehr wohl als eine intersubjektiv nachvollziehbare Prozedur bezeichnen, denn was könnte "intersubjektiver" als Zählen sein. Eine adäquate Ope- 
rationalisierung des intuitiven Explikandums '(relativer) Dekompositionsgrad' stellt diese Prozedur jedoch nicht dar. Dem quantitativen Vergleich der Seme, aufgrund dessen von zwei gegebenen Bedeutungsexplikationen desselben Stichworts entschieden werden soll, ob sie bezüglich ihres Dekompositionsgrades vergleichbar sind, stehen die beiden folgenden oberlegungen entgegen:

1) Vergleichen wir zwei gegebene lexikographische Definitionen desselben Stichworts hinsichtlich der Anzahl ihrer Seme, um uber ihren relativen Dekompositionsgrad eine Aussage treffen zu können, so verkennen wir den Zeichencharakter, der die Seme, die in der linguistischen Semantik angesetzt werden, auszeichnet. Diese Eigenschaft kommt allen Semen zu, ganz gleichgültig, ob sie als Teile der naturlichen Sprache wie im Wörterbuch von OZEGOV oder als Teile einer Kunstsprache wie im EKW angesehen werden. Unsere Fragestellung zielt auf den Grad ab, mit dem zwei gegebene lexikographische Definitionen die Bedeutung desselben Stichworts in ihre feineren Bedeutungskomponenten zerlegen, oder, m.a.W., auf die Informativität, mit der die betrachteten lexikographischen Definitionen Angaben zur Struktur der explizierten Lexembedeutung machen. Wir interessieren uns also für eine Erscheinung, die auf einer Abstraktionsebene betrachtet werden muB, die wir als die Inhaltsseite des Definiens, genauer gesagt, der dort verwendeten Seme bezeichnen wollen. Untersuchen wir die Seme auf die oben genannte Weise quantitativ, so nehmen wir jedoch einen anderen Blickwinkel ein. Indem wir die Wörter des Texts der lexikographischen Definition abzählen, erfassen wir nämlich nicht, wie für einen Vergleich der Dekompositionsgrade von Bedeutungsexplikationen erforderlich, die Seminhalte, sondern nur deren Ausdrücke. Die linguistische Ebene, auf der wir uns bewegen, ist somit die Ausdrucksseite der Seme. Da der Dekompositionsgrad von Bedeutungsexplikationen desselben Stichworts ein Phänomen ist, das der Inhaltsebene der in ihnen benutzten Seme zuzuweisen ist und folglich dort beschrieben werden muB, dürfen wir die kriterien, mit deren Hilfe wir über diese Erscheinung Aussagen machen wollen, auch nur auf dieser Abstraktionsebene gewinnen. Soweit wir den 
heutigen Forschungsstand der linguistischen Semantik überblicken, erscheint es uns allerdings aussichtslos, zu solchen Kriterien zu gelangen. Für diese miBliche Lage muB die Nichttranszendierbarkeit der Sprache verantwortlich gemacht werden, die sich auf metasprachliche semantische Deskriptionen voll auswirkt (vgl. S. 38 f.). Solange die Inhalte von Semen, die in semantischen Beschreibungen Verwendung finden, nicht hinreichend spezifiziert werden, wird es daher für den Linguisten unmöglich sein, über die Vergleichbarkeit von lexikographischen Definitionen desselben Stichworts bezüglich des Grades, mit dem sie dessen Bedeutung dekomponieren, Aussagen zu machen, die intersubjektiv kontrollierbar sind, al so nicht auf der Introspektion des Analysators beruhen.

Am Beispiel der oben genannten Bedeutungsexplikationen von udarjats läßt sich die Problematik gut veranschaulichen, die introspektives Vorgehen für Ermittlung und Vergleich von Dekompositionsgraden semantischer Repräsentationen mit sich bringt. Betrachten wir die beiden lexikographischen Definitionen, so erscheint es uns aufgrund der Inhalte, die mit den Semen assoziiert werden können, intuitiv naheliegend, zwischen ihnen u.a. die folgenden (Sinn)Relationen anzusetzen:

1. 'Proizvodit' tolčok' (OŽEGOV) impliziert immer eine Berührung des Gegenstands, in dessen Richtung die Handlung des StoBens erfolgt. Der Ausdruck ist daher Hyponym von 'prikasat'sja k čemu-n.' (OŽEGOV) und von 'privodit' čto-libo $v$ kontakt $s$ čem-libo' (EKW)

2. Die Bedeutungskomponenten 'prikasat'sja $k$ čemu-Zibo' (OZEGOV) und 'privodit' čto-n. $v$ kontakt $s$ čem-n. (EKW) betrachten wir als Synonyme, obwohl sie sich zumindest qualitativ im Stellenplan der semantischen Valenzen unterscheiden, die OZEGOV bZw. das EKW ihnen zuweisen. Die Frage, wie Unterschiede in der Rollenstruktur von Bedeutungsexplikationen desselben Stichworts im Hinblick auf ihren relativen Dekompositionsgrad zu bewerten sind, wollen wir an dieser stelle sowie bei unseren oberlegungen unter 2) (s.u.) unberücksichtigt lassen. Das Dilemma, in dem die linguistische Semantik bei der "Auffindung" von Semen und Kasusrollen steckt, 
läßt eine befriedigende Lösung dieses Problems zur Zeit kaum erwarten ( $v g l$. S. 17). Es ist übrigens durchaus möglich, für die Seme 'prikasat'sja $k$ čemu-n.' (OZEGOV) und 'privodit' čto-n. $v$ kontakt s čem-n.' (EKW), die wir oben synonym gesetzt haben, die unterschiedlichen Rollenstrukturen quantitativ anzugleichen, da der Inhalt, den wir mit "prikasat'sjak čemu-n.' (OZZEGOV) assoziieren, auch eine Stelle für einen Mitspieler umfaßt, der die Rolle eines Instruments spielt; vgl. prikasat'sja $k$ stolu ladon'ju, rukoj, palkoj, rukavom.

3. 'Proizvodit' tolčok' (OŽEGOV) ist Synonym von 'kratkovremenno privodit' čto-n. v kontakt s čem-n. ' (EKW). Oie Handlung des Stoßens impliziert immer eine Berihrung, die nur kurze Zeit ('kratkovremenno') andauert. Dieses Sem ist fiur die Bedeutung von 'proizvodit' tolčok' (OŽEGOV) paradigmatisch, da bei der Handlung des Stoßens zwischen den beiden sich berührenden Gegenständen eine sofortige Trennung erfolgt.

4. '(... prikasaetsja) s siloj (k stolu)' (OZEGOV) ist Synonym von '(Učitel') rezko (privodit...) (EKW).

5. '(... proizvodit) rezkij (tolcook ...)' (OŽEGOV) ist Synonym von '(Učitel') rezko (privodit...)' (EKW).

6. Aus den unter 4. und 5. aufgeführten Aquivalenzen folgt, daB ' (... prikasaetsja) s siloj (kstolu)' (OŽEGOV) Synonym von ' (... proizvodit) rezkij (tolčok ....)' (OZ̈EGOV) is t.

Im Hinblick auf die oben genannten Relationen, die wir zwischen einzelnen Semen bzw. Semkomplexen der beiden betrachteten Bedeutungsexplikationen postulieren können, missen wir der lexikographischen Definition von OŽGOV einen weit geringeren Grad der Dekomposition der Bedeutung von udarjat' zuschreiben als der entsprechenden Definition des EKW, da erstere die redundanten Seme 'prikasat'sja $k$ stolu' und 's $8 i z o j '$ bzw. 'rezkij' enthält. Assoziieren wir jedoch mit der Bedeutungskomponente 'proizvodit' tolčok' (OZZEGOV) einen anderen Inhalt, so müssen wir zwischen den Semen der beiden lexikographischen Definitionen auch andere Relationen ansetzen. Es scheint intuitiv ebenso naheliegend zu sein, da $B$ 
'proizvodit' tolčok' nicht unbedingt eine Berührung des Gegenstands impliziert, in dessen Richtung die Handlung des Stoßens erfolgt. Weisen wir dem Sem diese semantische Interpretation zu, durfen wir weder eine Hyponymie- noch eine Synonymierelation zu den Bedeutungskomponenten 'prikasat'sja $k$ čemu-n.' (OZZEOV) bZw. 'kratkovremenno privodit' čto-n. v kontakt 8 čem-n.' (EKW) ansetzen. ${ }^{38}$ In diesem Fall sehen wir 'proizvodit' tolčok' (OZEGOV) in attributiver Verknüpfung mit 'prikasat'sja $k$ čemu-n.' (OŽEGOV) als eine Bedeutungskomponente an, die für die Bedeutung von udarjat' paradigmatisch ist. Unsere Interpretation der Bedeutungskomponente hat zur Folge, daB die oben erwähnte Semredundanz in der Bedeutungsexplikation von OZEGOV sich verringert, während der relative Dekompositionsgrad der OZEGOVschen Definition zur lexikographischen Definition des EKW wächst.

2) Gegen den oben skizzierten quantitativen Vergleich der Anzahl der Seme von Bedeutungsexplikationen desselben Stichworts muB ein weiterer Einwand erhoben werden, der mit 1) eng verbunden ist. Während sich unsere erste oberlegung auf bestimmte semiotische Eigenschaften der Seme als isolierter Einheiten oder "Wörter" des Texts der lexikographischen Definiton bezieht, betrifft unser zweiter Vorbehalt die Art der syntaktischen Relationen, mit denen die Seme der Bedeutungsexplikation untereinander verknüpt sind. Eine Gegenüberstellung der oben genannten wortsprachlichen semantischen Repräsentationen von udarjat' zeigt, daß sie syntaktisch verschieden strukturiert sind. Mit Hilfe des (Quasi) Formalismus, den APRESJAN als alternative Notation zur wortsprachlichen formulierung der SemS vorschlägt (vgl. S. 11 f.), lassen sich die syntaktischen Unterschiede zwischen den beiden Bedeutungsexplikationen gut darstellen:

(10) udarjat' (EKW)

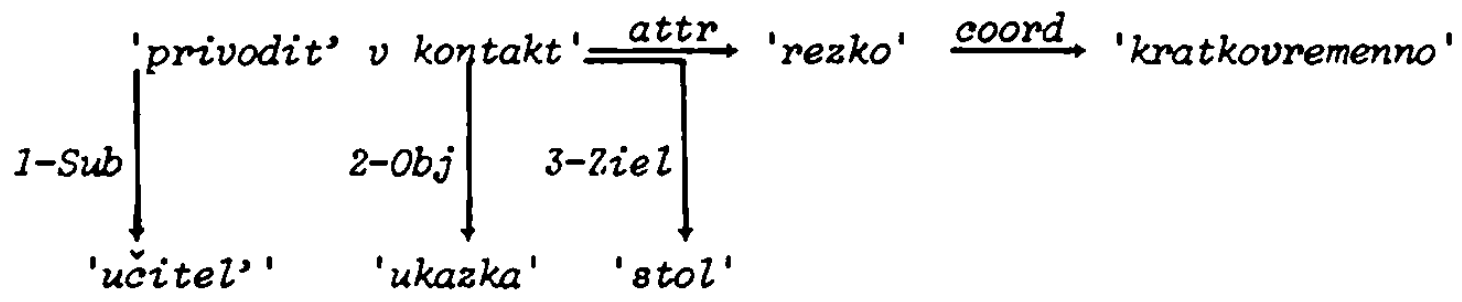


00050464

$-160-$

(11) udarjat' (OZEGOV)

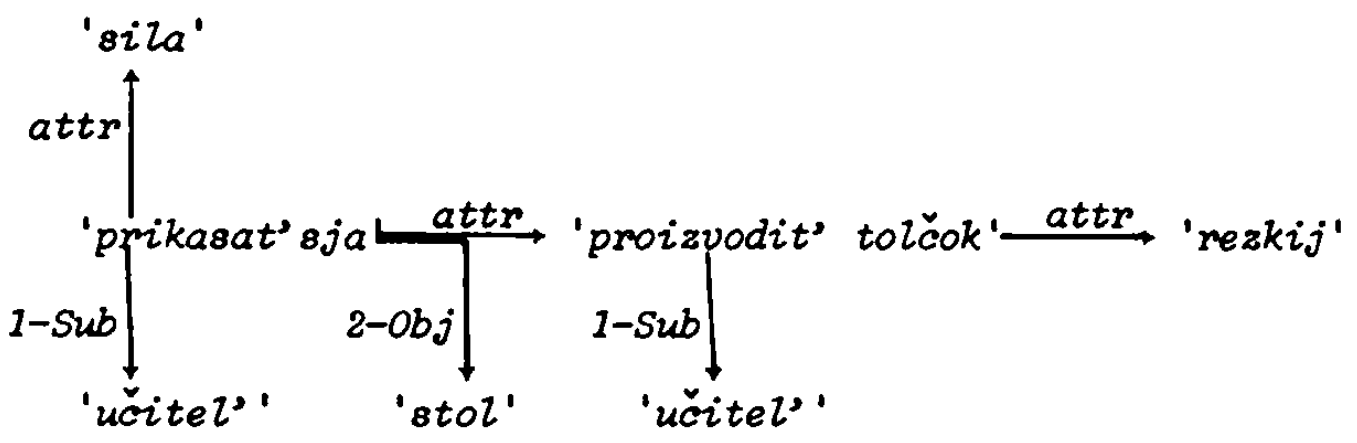

Die unterschiedlichen syntaktischen Strukturierungen, welche lexikographische Definitionen desselben Stichworts aufweisen können, lassen eine weitere Schwierigkeit zutage treten, die sich bei dem Versuch ergibt, die Vergleichbarkeit der Bedeutungsexplikationen hinsichtlich ihres Dekompositionsgrades auf andere als intuitive Art zu ermitteln. Wir können nicht intersubjektiv kontrollierbar feststellen, inwieweit syntaktische Relationen, die zwischen den Semen der zu vergleichenden lexikographischen Definitionen angesetzt werden, für den Grad verantwortlich zu machen sind, mit dem die semantischen Repräsentationen die Bedeutung des Stichworts dekomponieren. Es ist unmöglich, für das oben genannte Beispiel auf intersubjektive Weise zu beurteilen, ob die Bedeutungsexplikation (10) die Bedeutung von udarjat' stärker dekomponiert als die Bedeutungsexplikation (11).

Wollen wir den Versuch unternehmen, Aussagen über den Beitrag zu machen, den syntaktische Relationen zwischen den Semen von lexikographischen Definitionen für deren relativen Dekompositionsgrad leisten, so setzt das die Kenntnis der Inhalte der Seme voraus. Da es sich bei den Semen - wie bereits besprochen - per definitionem um axiomatische GröBen handelt und wir daher auch nicht auf Semexplikationen zuriuckgreifen können, sind wir, wie oben gezeigt, darauf angewiesen, mit Semen Inhalte rein intuitiv zu assozijeren. Den Beitrag der syntaktischen Relationen zwischen den Bedeutungskomponenten einer semantischen Repräsentation für deren Dekompositionsgrad betrachten wir folglich in Abhängigkeit von den Seminhalten. Interpretieren wir beispielsweise bestimmte Bedeutungskom-

Klaus Hartenstein - 9783954792764

Downloaded from PubFactory at 01/10/2019 05:16:44AM

via free access 
ponenten einer lexikographischen Definition als redundant (s.o.), düfen wir den syntaktischen Relationen, die die betreffenden Seme miteinander verknüpfen, keine Relevanz für den Dekompositionsgrad der Bedeutungsexplikation zuweisen. Interpretieren wir dagegen die Bedeutungskomponenten einer lexikographischen Definition so, daß keine Semredundanz anzusetzen ist, sind wir gezwungen, die "Leistung" zu bewerten, die die syntaktischen Relationen zwischen den Semen der Bedeutungsexplikation fur deren Dekompositionsgrad erbringen.

Anhand der syntaktischen Relationen Attribution und Koordination wollen wir demonstrieren, auf welche Weise das geschehen kann. In unserer Betrachtung der beiden Relationen beschränken wir uns dabei ganz bewuBt auf ihre Verwendung in (der lexikographischen Variante) der SemS des EKW. Diese bietet nämlich den Vorteil, ihre Syntax stark zu reglementieren, so daß im Vergleich zur Definitionssprache von OŽEGOV relativ wenig verschiedene Ausdrucksmittel benutzt werden, um das Vorhandensein einer Attributions-bzw. Koordinationsrelation zwischen den Semen ihrer Bedeutungsexplikationen zu signalisieren ( $v g l$. S. $60 \mathrm{f.})$. Ein weiteres Ziel unserer Untersuchung, das sich aus der Frage nach dem Beitrag von syntaktischen Relationen zwischen Semen für den relativen Dekompositionsgrad konkurrierender lexikographischer Definitionen zwangsläufig ergibt, besteht darin, zu präzisieren, wie die Konstrukteure des MST die Attributions- und die Koordinationsrelation zwischen Semen bei der semantischen Beschreibung einsetzen. Dies ist erforderlich, weil ihre Ausfihrungen zur Syntax der SemS des EKW leider nicht geeignet sind, daruber in befriedigender Weise AufschluB zu geben. Die Konstrukteure des MST beschränken sich nämlich, zumindest was die lexikographische Variante der Sems angeht, in ihren Publikationen darauf, das Inventar an Relationen der Syntax anzugeben, ohne auf deren Verwendung in der semantischen Deskription einzugehen (für die Verwendung der Attributions-und Koordinationsrelation in der Metasprache, die auf der Ebene der semantischen Repräsentation im MST benutzt wird, vgl. S.61).

Wir betrachten nun die lexikographische Definition (12), die 
das EKW für das Verb ubě̌dat' (in einer seiner Bedeutungen) gibt:

(12) $\frac{u b e \check{z} d a t^{\prime}}{x u b e \check{z} d a}$

$X$ ubeždaet $Y-a \vee 2-e=$ 'Privodja argumenty, $X$ pytaetsja kauzirovat' $Y$-a sčitat' $2 . '$ (APRESJAN 1974b, 83)

In dieser Bedeutungsexplikation wird zwischen den Semen 'privodit' argumenty' und 'pytat'sja' eine attributive Verknüpfung angesetzt. Wir müssen zumindest aufgrund der Beschreibungspraxis im MST das grammatische Mittel der präsentischen Gerundialkonstruktion als ein Ausdrucksmittel interpretieren, das in der wortsprachlichen (= lexikographischen) Notationsvariante der Sems ublicherweise verwendet wird, um eine Attributionsrelation zu signalisieren. Da wir den Beitrag erfassen wollen, den Attributions- und Koordinationsrelation für den relativen Dekompositionsgrad von Bedeutungsexplikationen desselben Stichworts leisten, verändern wir nun die lexikographische Definition (12), indem wir die Seme 'privodit' argumenty' und 'pytat'sja' mit Hilfe der Kopula ' $i$ ' verbinden, die in der lexikographischen Variante der sems als Ausdrucksmittel für eine koordinative Verknüpfung verwendet wird:

(13) $\frac{u b e \check{z} d a t^{\prime}}{x \text { ube } \check{z} d a e t}$

$X$ ubě̃daet $Y-a \vee 2-e=' X$ privodit argumenty $i$ pytaetsja kauzirovat' $y-a$ sčitat' 2 .'

Außerdem nehmen wir an, daß die Bedeutungskomponenten, die in (12) und (13) angesetzt werden, uns bekannt und invariant sind. Erst diese Voraussetzung erlaubt es uns, eventuell den Beitrag von Attributions - und Koordinationsrelation für den relativen Dekompositionsgrad der beiden konkurrierenden Bedeutungsexplikationen von ubeždat' zu erfassen. Es ist für unsere Zwecke völlig ausreichend, daß wir die Inhalte, die wir mit den Semen 'privodit' argumenty' und 'pytat'sja' assoziieren, als den zweier handlungsbezeichnender Prädikate spezifizieren. Die Frage, die wir im Hinblick auf die beiden lexikographischen Definitionen zu beantworten haben, besteht somit darin, herauszufinden, ob diese beiden Prädikate in 
Verbindung mit den anderen Bedeutungskomponenten in ihrer syntaktischen Anordnung in

(12) 'Privodit' argumenty' (X) $\stackrel{\text { attr }}{\longrightarrow}$ 'pytat'sja'(x, 'kauzirovat' $(X$, 'sčitat'' $(Y, Z)))$

die Bedeutung des Stichworts stärker dekomponieren als in ihrer syntaktischen Verknüpung in

(13) 'Privodit' argumenty' $(X) \wedge$ 'pytat'sja' (X, 'kauzirovat'. $(X$, 'scitat' $(Y, Z)))$.

Um diese Frage zu Iösen, müssen wir die Aufgaben kennen, die der Attributions- und der Koordinationsrelation zwischen Semen in Bedeutungsexplikationen zukommen. Sie werden jedoch, wie bereits erwähnt, von den Konstrukteuren des MST nicht genannt. Wir sind daher gezwungen, aus den lexikographischen Definitionen des EKW die Verwendungsweise der Attributionsund der Koordinationsrelation zu erschließen. Dabei sind wir wiederum auf introspektives Vorgehen angewiesen.

Bei unserer Untersuchung lassen wir uns von den Oberlegungen leiten, welche wir zum Status der SemS des EKW als einer Kunstsprache angestellt haben. Wir gehen davon aus, daB es sich bei der Sems um die in ihren syntaktischen und lexikalischen Ausdrucksmitteln stark reglementierte natürliche Sprache Russisch handelt, in der jedem syntaktischen Ausdrucksmittel genau eine invariante Verknüpfungsfunktion und jedem Wort genau ein invarianter Inhalt zukommt. M.a.W., wir beziehen uns, indem wir diese Voraussetzung machen, auf das Postulat der Konstrukteure des MST, daB zwischen den Ausdrucksmitteln der Sems und den durch sie signalisierten Inhalten die Relation der Ein-Eindeutigkeit zu bestehen habe. 39

Ausgehend von dieser Prämisse untersuchen wir nun, wie das präsentische Gerundium und die Kopula $\underline{i}$ in der naturlichen Sprache Russisch verwendet werden. Besonders im Hinblick auf die Kopula $\underline{i}$ ist es wichtig, daB wir ihre Verwendungsweise in der natürlichen Sprache innerhalb der gleichen Umgebung untersuchen, die sie auch in der sems hat. Für die Kopula $\underline{i}$ ist dies die kategoriale Umgebung VP_VP. Wir assoziieren nun mit 
den hier in Rede stehenden Ausdrucksmitteln, die in der Sems verwendet werden, um eine attributive bzw. eine koordinative Relation zwischen Semen zu signalisieren, diejenigen semantischen Funktionen, welche sie in der natijrlichen Sprache haben. Unter der semantischen Funktion der präsentischen Gerundialkonstruktion verstehen wir die Signalisierung einer Bedeutungsrelation, die $z$ wischen den beiden Handlungen besteht, welche durch das Gerundium und das Hauptverb des Satzes, in dem sie vorkommen, ausgedrückt werden.

Unter der semantischen Funktion der Kopula $\underline{i}$ verstehen wir die Signalisierung einer Bedeutungsrelation, die die Kopula zwischen den beiden Handlungen stiftet, welche durch die Verben bezeichnet werden, die sie miteinander verknupft. Wir betrachten nun die Sätze (14) - (17):

(14) Opirajas' na konduktora, Ženja vychodit iz avtobusa.

(15) Sidja v sadu, Larisa pišet Žene otkrytku.

(16) Larisa rasstilaet skatert' i nakryvaet na stol.

(17) Zenja kurit i smotrit televizor.

Aufgrund der semantischen Funktion, die wir mit den präsentischen Gerundialkonstruktionen in (14) und (15) assoziieren, interpretieren wir die durch sie signalisierten Bedeutungsrelationen zwischen den Handlungen opirat'sja (Zenja, konduktor) und vychodit' (Zenja, avtobus) bzw. sidet' (Larisa, sad) und pisat' (Larisa, Kenja, otkrytka) als Modifikationen oder Spezialisierungen, wobei die gerundial ausgedruckten Handlungen als modifizierende, die durch die finiten Verbformen ausgedrückten Handlungen als modifizierte aufzufassen sind. Aus der Bedeutungsrelation der Modifikation, in der die genannten Teile von (14) und (15) stehen, folgt, daB der Sprecher beide Handlungen jeweils als gleichzeitig ablaufend darstellt.

Weisen wir dem präsentischen Gerundium die semantische Funktion zu, zwischen zwei Teilsätzen die Bedeutungsrelation der Modifikation oder Spezialisierung zu stiften, so missen wir der Kopula $\underline{i}$ eine andere semantische Funktion zuschreiben. Hierzu sind wir deswegen gezwungen, weil wir, wie bereits erwähnt, bei unserer Untersuchung der Aufgabe, die den syntak- 
tischen Relationen der Attribution und der Koordination bei der semantischen Beschreibung zufallen, von dem Postulat der Konstrukteure des MST ausgehen, daB zwischen den Inhaltsund Ausdrucksseiten der Ausdrucksmittel der SemS des EKW als einer im besonderen Maße reglementierten Form des Russischen die Beziehung der gegenseitigen Eindeutigkeit zu bestehen habe (s.o.). Die semantische Funktion, die wir mit der Kopula $\underline{i}$ in (16) und (17) assoziieren, wollen wir daher - sozusagen ex negativo - als die Signalisierung einer Nichtmodifikations- oder Nichtspezialisierungsverknüpung angeben. In (16) und (17) werden Ablauf und Umstände der von nakryvat, na stol (Larisa) bzw. smotret' ('̌enja, televizor) bezeichneten Handlung durch rasstizat' (Larisa, skatert') bzw. kurit' (Zenja) nicht modifiziert, so daß auch die zeitliche Zuordnung der Handlungen im Gegensatz zur Modifikation unerheblich ist.

Nachdem wir, ausgehend von der Forderung der Konstrukteure des MST nach Ein-Eindeutigkeit der Zuordnung von Inhalt und Ausdruck in der SemS des EKW, unter Rekurs auf die natürliche Sprache herausgefunden haben, welche semantische Funktionen der präsentischen Gerundialkonstruktion und der Kopula $\underline{i}$ (in einer bestimmten Umgebung) als Ausdrucksmittel der wortsprachlichen Variante der Sems für eine Attributions-bzw. Koordinationsrelation zwischen Bedeutungskomponenten in einem auf diese Weise reglementierten Russisch zuzuschreiben sind, können wir nun versuchen, die Frage zu beantworten, wie der relative Dekompositionsgrad zweier konkurrierender lexikographischer Definitionen desselben Stichworts durch sie affiziert wird.

Dazu betrachten wir noch einmal die beiden oben genannten Bedeutungsexplikationen von ube żdat', die sich nur dadurch unterscheiden, daB zwischen den Semen 'privodit' argumenty' und 'pytat'sja' eine attributive bzw. eine koordinative syntaktische Relation angesetzt wird. Aufgrund der semantischen Funktion, die wir für die Attributionsrelation zwischen Semen ermittelt haben, müssen wir der lexikographischen Definition (12) einen relativ zu der lexikographischen Definition 
höheren Dekompositionsgrad zuweisen. Für die von ubeždat' bezeichnete Situation ist es paradigmatisch, daB der Mitspieler, dem die Agensrolle zukommt, versucht, beim Adressaten eine Meinungsänderung herbeizufiuhren, indem er gleichzeitig, um dieses Ziel zu erreichen, Argumente vorbringt. Um diesen Sachverhalt zu beschreiben, wird in der semantischen Repräsentation von uberádat' mit Hilfe der Attributionsrelation angegeben, daB die durch 'privodit' argumenty' $(X)$ ausgedrückte Handlung den durch 'pytat'sja' ( $x$, 'kauzirovat' $(x$, 'sčitat' $(y, z)))$ bezeichneten Handlungskomplex modifiziert.

Diese Bedeutungsrelation wird in der lexikographischen Definition (13) nicht abgebildet. Obwohl sie wie (12) alle für das Stichwort paradigmatischen Seme umfaBt, dekomponiert sie die Bedeutung des Stichworts in geringerem AusmaB, da sie eine Bedeutungsrelation zwischen diesen Semen, die eine wichtige Denotationsbedingung für ubězdat' darstellt, unberücksichtigt läBt und somit über die Struktur der zu explizierenden Lexembedeutung weniger Angaben macht.

Es erscheint uns erforderlich, im Zusammenhang mit den Problemen, auf die man bei der Ermittlung des relativen Dekompositionsgrades konkurrierender lexikographischer Definitionen stößt, kurz die Frage nach dem Verhältnis zwischen Dekompositionsgrad, d.h. der Menge an Informationen, die die Bedeutungsexplikationen uber die zu definierende Lexembedeutung geben, und deren Güte, d.h.der Vollständigkeit, mit der sie die Lexembedeutung explizieren, anzusprechen. Aus dem oben genannten Beispiel ubězdat' wird deutlich, daB eine Entscheidung zugunsten der semantischen Repräsentation (12) uns nicht nur eine Beschreibung liefert, die die Bedeutung des Stichworts relativ zu (13) stärker dekomponiert und daher informativer ist, sondern sie auch besser abbildet. Wir durfen hieraus jedoch keinesfalls ableiten, daB diejenige von zwei gegebenen lexikographischen Definitionen desselben Stichworts dessen Bedeutung adäquater beschreibt, die, vorausgesetzt, er läßt sich überhaupt auf befriedigende Weise bestimmen, den relativ höheren Dekompositionsgrad aufweist. Einer solchen Korrelierung von Gite und Dekompositionsgrad 
lexikographischer Definitionen widerspricht die Art und Weise unseres Vorgehens. Wir sind, um herauszufinden, welche Funktion der Attributions- bzw. der Koordinationsrelation bei der semantischen Beschreibung durch die SemS des EKW zufällt und welchen Beitrag diese Relationen demzufolge für den relativen Dekompositionsgrad der Bedeutungsexplikationen (12) bzw. (13) leisten, von der Prämisse ausgegangen, da $B$ uns in beiden Fällen die Inhalte der Seme bekannt sind und ihr Bestand invariant bleibt. AuBerdem muBten wir alle syntaktischen Relationen zwischen den Bedeutungskomponenten der lexikographischen Definitionen bis auf eine unverändert lassen. M.a.W., wir haben uns einen Idealfall konstruiert, indem wir mit einer Ausnahme von allen faktoren, die zur Tiefe der Dekomposition einer Bedeutungsexplikation beitragen, abstrahiert haben.

Kehren wir nun zum Ausgangspunkt unserer Oberlegungen zurück. Diese bestanden darin, daß wir zwischen den lexikographischen Definitionen, die das EXW bzw. das Wörterbuch von OZEGOV für dasselbe Stichwort (in einer seiner Bedeutungen) geben, nur annähernde Vergleichbarkeit herbeiführen können. Zwar läßt sich - wie oben gezeigt - nachweisen, daß der relative Dekompositionsgrad konkurrierender Bedeutungsexplikationen sowohl von den Inhalten, der den in ihnen verwendeten Semen zugeschrieben wird, als auch von der Art deren syntaktischer Anordnung abhängig ist. Es bleibt jedoch letztlich dem intuitiven Urteil eines jeden einzelnen Analysators überlassen, diese Abhängigkeiten zu bestimmen. Aus diesem Grund stehen uns auch keine intersubjektiv nachvollziehbaren Kriterien zur Verfügung, die es uns erlaubten, die Vergleichbarkeit von konkurrierenden Bedeutungsexplikationen bezüglich ihres relativen Dekompositionsgrads auf "objektive" Weise zu beurteilen.

Neben der prinzipiellen Schwierigkeit, die der Vergleich beliebiger semantischer Beschreibungen hinsichtlich des Grades, in dem sie die Bedeutung einer gegebenen sprachlichen Einheit dekomponieren, mit sich bringt, stoßen wir bei der Konstruktion unseres Tests noch auf ein technisches Problem, für das das Wörterbuch von OZEGOV verantwortlich zu machen ist. 
Wir haben bereits auf das lexikologische Prinzip der Küze der Bedeutungsexplikation (kratkost' tolkovanija) hingewiesen, dem OZZEGOV seine lexikographischen Definitionen aus Grijnden der Beschreibungsökonomie unterwirft. OZEGOV selbst betont jedoch, daB ohne weiteres die Möglichkeit bestehe, aufgrund der Struktur seiner Bedeutungsexplikationen die Folgen zu beheben, die aus der Anwendung dieses Prinzips resultierten. Es scheint, nimmt man OZZEGOV beim Wort (vgl. insbesondere $\S 14$ der "Explikation der Wortbedeutung" in OZEGOV $1973,8)$, daß der relativ zu den lexikographischen Definitionen des EKW geringere Dekompositionsgrad seiner Bedeutungsbeschreibungen ohne Schwierigkeiten zu erhöhen ist, indem man sie auf die bereits angedeutete Weise expandiert ( $v g l$. S. $153 \mathrm{f.}$.).

Betrachtet man die lexikographischen Definitionen des Wörterbuchs von OZEGOV, stellt man allerdings sogleich fest, daB es häufig unmöglich ist, die Definientes der Bedeutungsexplikationen auch nur ein einziges Mal zu expandieren, ohne daB sich Zirkularität in den Definitionen einstellt. Die folgenden Beispiele lexikographischer Definitionen, die OZEGOV für Wörter gibt, die auch das EKW expliziert, mögen dies verdeutlichen:

(18) prostit' (čto-n. komu-n.) = 'Ne postavit' v vinu čego-n., izvinit' '

$$
\text { (OZEGOV 1973, 572) }
$$

izvinit'

(19) udalit'sja

$\underline{0 t o j t i}$

(20) mokryj
= 'Prostit' '

(OZEGOV 1973, 221)

' 'otdalit'sja, otojti na

kakoe-n. rasstojanie'

(OZ̈EGOV 1973, 758)

='Pojti, udalit'sja ot kakogo-n. mesta'

(OZEGOV 1973, 435)

='syroj, viažnyj, soveršenno propitavšij vlagoj

(OZEGOV 1973, 328) 
syroj

$\underline{v \text { Lǎ̃nyj }}$

(21) Zovkij

snorovka
' 'Vlažnyj, ne suchoj'

(OZ̈EGOV 1973, 722)

'Propitannyj viagoj, mokryj, syroj'

(OZ̈EGOV 1973,80)

= 'Iskusnyj, obladajuščij

fizičeskoj snorovkoj'

(OŽEGOV 1973, 301)

= 'Lovkost', umenie $v$ ka-

kom-n. dele'

(OŽEGOV 1973,679$)^{40}$

Aus diesen Beispielen wird deutlich, daB im Hinblick auf tautologische Wörterbuchdefinitionen OZZEGOVs lexikographische Praxis dessen lexikologischen Erwägungen zur Struktur der Bedeutungsexplikation nicht nachkommt.

Besonders verwunderlich sind die Ausfuhrungen von SVEDOVA, die im Vorwort zum Wörterbuch von OZZEGOV u.a. die Frage tautologischer Zirkel anspricht. Sie behauptet, daB im Zuge einer gründlichen Oberarbeitung der 10. Ausgabe des Wörterbuchs Tautologien zwar nicht völlig vermieden, jedoch stark reduziert worden seien:

"Sog. 'zirkuläre Bedeutungsexplikationen', d.h. die Erklärung eines Wortes durch dasjenige Wort, welches seinerseits durch ersteres expliziert wird, sind überall dort, wo es erforderlich und möglich war, entfernt worden." (OZZEGOV 1973, 9)

Mit dieser Feststellung begibt sich SVEDOVA ganz offensichtlich in Widerspruch zur Beschreibungspraxis des Wörterbuchs. Interessant düfte es auch sein, in Erfahrung zu bringen, was sie unter einer tautologischen Definition versteht und unter welchen Umständen sie die Beseitigung von Tautologien für erforderlich und für möglich hält.

Zwar kann man nicht davon ausgehen, daß das Vorhandensein zirkulärer Bedeutungsexplikationen in einem nichtfachsprachlichen einsprachigen Definitionswörterbuch, sofern bestimmte von uns bereits besprochene Voraussetzungen erfiillt sind (vgl. S. $77 \mathrm{ff.}$, dessen praktischen Nutzwert für seine Be- 
nutzer unbedingt schmälern muß. Für die Konstruktion unseres Testmaterials und die Durchführung unseres Versuchs ergeben sich aus diesem Umstand jedoch negative Konsequenzen.

\subsection{Prinzipien der Konstruktion des Testmaterials}

Das Vorhandensein tautologischer Definitionen im Wörterbuch von OŽEOV hat Beschränkungen für den Umfang unseres Test zur Folge, weil wir nicht alle Bedeutungsexplikationen von Lexemen, die das EKW bisher zur Verfugung stellt, in unseren Versuch miteinbeziehen durfen. Wir können nur diejenigen lexikographischen Definitionen des EKW berücksichtigen, denen im Wörterbuch von OZEGOV Bedeutungsbeschreibungen entsprechen, die entfaltet werden können. Als Anforderung an die Expandierbarkeit der Bedeutungsexplikationen von OŽEGOV legen wir fest, $d a B$ diese sich mindestens einmal entfalten lassen muissen, ohne tautologisch (s.u.) zu werden.

Unter der Expandierung (oder Entfaltung) einer lexikographischen Definition verstehen wir eine Paraphrasierung ihres Definiens, die den folgenden formalen und inhaltichen Voraussetzungen genügen muß:

1. In formaler Hinsicht muB gewährleistet sein, daB ein Sem, das im Definiens einer gegebenen lexikographischen Definition verwendet wird, durch nicht weniger als zwei andere, von ihm ausdrucksverschiedene Bedeutungskomponenten substituiert werden kann.

2. Die Substitution muB auBerdem die inhaltliche Voraussetzung erfillen, daB die Bedeutung des substituierten Sems von den Semen, durch die es ersetzt worden ist, ohne Redundanz dekomponiert wird.

Am Beispiel der Bedeutungsexplikation, die das Wörterbuch von OŽEGOV für eine der Bedeutungen von arestovyvat' gibt, wollen wir die Expandierung einer lexikographischen Definition demonstrieren. OZEGOV definiert wie folgt:

(22) arestovyvat' = 'Podvergat' arestu kogo-n.' (OZEGOV 1973, 30) 
(23) $\underline{\text { arest }}=$ 'Zakijučenie pod stražu' (OZEGOV 1973, ebd.)

Paraphrasieren wir die Bedeutungsexplikation (22), indem wir die Bedeutungskomponente 'arest' durch die Seme 'zakzjučenie pod stražu' ersetzen, haben wir die lexikographische Definition des Stichworts entfaltet, da die Operation sowohl der formalen als auch der inhaltlichen Bedingung genügt, die erfüllt sein müssen, damit man von einer Expandierung der Bedeutungsexplikation sprechen kann. Bei dem Semkomplex 'zaključenie pod stražu' handelt es sich um nicht weniger als zwei, von dem Sem 'arest' ausdrucksverschiedene Bedeutungskomponenten. Sie dekomponieren dessen Bedeutung ohne Redundanz in die Teilbedeutungen 'zaključenie' und 'pod stražu', da der Begriff 'arest' eben diese Teilbegriffe ('Freiheitsberaubung' bzw. 'Festsetzung einer Person' und 'Oberwachung dieser Person') impliziert.

Im Gegensatz dazu läßt sich beispielsweise die lexikographische Definition, die OZZEOV für blagodarnyj gibt, nicht weiter entfalten, da die Paraphrasierung eine der Bedingungen für eine Expandierung nicht erfüllt. OZZEOV definiert wie folgt:

(24) $\underline{\text { blagodarnyjj }=~ ' C u v s t v u j u s ̌ c ̌ i j ~ i l i ~ v y r a z ̌ a j u s ̌ c ̌ i j ~ b l a g o-~}$ darnost" (OZEGOV 1973, S. 48)

(25) blagodarnost' $=$ 'Uuvstvo priznatel'nosti $k$ komu-n. za okazannoe dobro, vnimanie' (OZEGOV 1973, ebd.)

Zwar kann in der Bedeutungsexplikation (24) von blagodarnyj das Sem 'blagodarnost" ' durch die beiden, von diesem ausdrucksverschiedenen Bedeutungskomponenten 'cuvstvo priznatel'nosti' substituiert werden:

(26) blagodarnyj $=$ '̌̌uvstvujuščij ili vyražajuščij čuvstvo priznatel'nosti k komu-n. za okazannoe dobro, vnimanie'

Der Semkomplex 'čuvstvo priznatel'nosti' zerlegt die Bedeutung von blagodarnost' jedoch nicht ohne Redundanz, weil die Bedeutungen von 'čuvstvo' und 'priznatel'nost' ' in einer besonderen Inklusionsbeziehung stehen. 
Dies läBt sich in Anlehnung an Oberlegungen, die APRESJAN (a)lerdings unter einer anderen als der uns hier interessierenden Fragestellung) zur semantischen Beschreibung von Wortverbindungen anstellt, gut veranschaulichen (vgl. S. $135 \mathrm{f.}$ ) Sowie APRESJAN 1974b,79ff. und GAK 1972). Bei der Klasse der Individuen, auf die wir mit Hilfe der Bedeutungen der Lexeme čuvstvo und priznatel'nost' referieren können, handelt es sich einmal um eine bestimmte Art von Referenten, nämlich die Klasse der Gefühle ('čuvstva'), zum anderen um eine bestimmte Spezies von Referenten innerhalb dieser Klasse, nämlich diejenigen Gefühle, die Dankbarkeit ('priznatel'nost') ausdrücken. Unter Berucksichtigung der semantischen Interpretation, die wir den Einzellemen čuvstvo und priznatel'nost' zugewiesen haben, begeben wir uns nun, um das Syntagma čuvstvo priznatel'nosti, das sich aus je einer Wortform dieser beiden Lexeme zusammensetzt, semantisch zu beschreiben, auf die Abstraktionsebene der Wortformverbindungen. Auf dieser Ebene können wir die Bedeutung der zweiten Wortform des Syntagmas priznatez'nosti als Differentia specifica zu einem Genus proximum anzusehen, das durch die Bedeutung der ersten Wortform des Syntagmas čuvetvo angegeben wird. 41 Zwischen den Bedeutungen der beiden Wortformen ouvstvo und priznatel'nosti mussen wir daher die Sinnrelation der Hyponymie ansetzen, d.h. wir interpretieren das Lexem priznatel'nost' als Hyponym des Lexems čuvstvo. Wenn zwischen den Bedeutungen zweier Lexeme $A$ und $B$ eine $\mathrm{Hy}-$ ponymierelation besteht, also $B$ Hyponym zu $A$ ist, ist die Bedeutung von $A$ in dér von $B$ völlig inkludiert. Werden Wortformen der Lexeme $A$ und $B$ in direkter syntaktischer Verknupfung zu einem Syntagma zusammengestellt, ergibt sich aufgrund ihrer semantischen Interpretation zwangsläufig Semredundanz (LYONS 1972, 463ff.; 1977, 291ff.; BREKLE 1972, 95ff.). Beziehen wir unsere oberlegungen auf das Beispiel der lexikographischen Definition, die OZEGOV fïr blagodarnyj anfïhrt, so ergibt sich folgendes Bild:

Definieren wir die Lexeme čuvstvo und priznatel'nost' wie folgt: 
(27) čuvstvo $='{ }^{\prime}$ uvstvo',

(28) priznatel'nost' $=$ 'Cuvstvo priznatel'nosti'

und nehmen wir auBerdem an, daB priznatel'nost' Hyponym zu cuvstuo ist:

(29) priznatel'nost' $\supset$ čuvstvo,

so enthält eine Definition, die wie beispielsweise bei OŽEGOV blagodarnyj u.a. mit Hilfe der Wortverbindung 'čuvstvo priznatel'nosti' expliziert, redundante Bedeutungskomponenten; vgl. die Expandierung von (24) zu (30) bzw. (31):

(24) $\underline{\text { blagodarnyj }}=$ 'Cuvstvujuščij ili vyražajuščij blagodarnost" (OZEGOV 1973, 48)

(25) blagodarnost' $=$ 'Cuvstvo priznatel'nostik komu-n. za ckazannoe dobro, unimanie' (OZEGOV 1973, ebd.);

also ist

(30) $\underline{\text { blagodarnyj }}=$ 'Cuvstvujuščij ili vyražajuščij čuvstvo priznatel'nosti k komu-n. za okazannoe dobro, vnimanie'

Aufgrund der Hyponymierelation, die wir zwischen den Lexemen čuvstvo und priznatel'nost' angesetzt haben (s.o.), folgt aus (30):

(31) $\underline{\text { blagodarnyj }}=$ 'Cuvstvujuščij ili vyražajuščij čuvstvo cuvstva priznatel'nosti k komu-n. za okazannoe dobro, unimanie'

Die Semredundanz 'čuvstvo čuvstva' in der Explikation (31) zeigt, daB eine Paraphrasierung von (24) in (25) die inhaltliche Bedingung nicht erfillt, die wir an die Expandierung einer lexikographischen Definition aus dem Wörterbuch von OZEGOV stellen. Die Bedeutungskomponenten 'cuvstvo priznatel'nost $i^{\prime}$, durch die wir in der gegebenen lexikographischen Definition (24) das Sem 'blagodarnost" substituiert haben, sind nicht in der Lage, dessen Bedeutung ohne Redundanz zu dekomponieren.

Das Beispiel der OZEGOVschen Bedeutungsexplikation von 
blagodarnyj verdeutlicht, daß es erforderlich ist, als definitorisches Kriterium für die Expandierung einer semantischen Beschreibung aus dem Wörterbuch von OZZEGOV nicht nur eine formale Bedingung festzulegen. Hätten wir uns darauf geeinigt, bereits dann von der Entfaltung einer gegebenen lexikographischen Definition zu sprechen, wenn es möglich ist, eine Bedeutungskomponente, die in dieser Bedeutungsexplikation verwendet wird, durch mindestens zwei von ihr ausdrucksverschiedene Bedeutungskomponenten zu ersetzen, so wären wir gezwungen, die Paraphrase (26) bzw. (30) der Bedeutungsexplikation (24) als deren Expandierung anzusehen. Dieser Interpretation widerspricht ganz offensichtlich der Definitionszirkel

priznatel'nost' $=$ 'Cuvstvo blagodarnosti'

$$
\text { (OZEGOV 1973,543), }
$$

der auch im Wörterbuch von OZ̈EGOV bei der Definition von priznatel'nost' zutage tritt. 42

Durch unsere Festlegung, nur dann eine Paraphrase einer gegebenen Bedeutungsexplikation als deren Expandierung zu betrachten, wenn sie den oben genannten formalen und inhaltlichen Voraussetzungen genügt, legen wir, wie bereits erwähnt, der Auswahl unseres Testmaterials Restriktionen auf. Unsere Definition der Expandierung einer gegebenen lexikographischen Definition aus dem Wörterbuch von OZZEGOV bietet allerdings den Vorteil, das Problem der Vergleichbarkeit konkurrierender Bedeutungsexplikationen bezüglich ihres Dekompositionsgrades einer Lösung zuzuführen, die wir als hinreichend für Aufbau und Durchführung unseres Experiments ansehen. Wir legen fest, daB jede lexikographische Definition aus dem Wörterbuch von OZ̈EGOV, vorausgesetzt, sie läBt sich mindestens einmal unter den oben genannten Bedingungen expandieren, mit der entsprechenden lexikographischen Definition des EKW hinsichtlich ihres Dekompositionsgrades verglichen werden kann, da sich schon bei einmaliger Entfaltung der OZ̈EGOVschen Bedeutungsexplikationen eine groBe Ahnlichkeit zwischen diesen und denen des EKW beobachten IäBt. Aus den bereits besprochenen Gründen ( $v g l$. S. 152 ff.) läßt sich 
auf diese Weise selbstverständlich nur eine annähernde Vergleichbarkeit der lexikographischen Definitionen bezuglich inres relativen Dekompositionsgrades erzielen.

Eine weitere wichtige Eigenschaft unseres Lösungswegs besteht darin, daß er operational ist, d.h., wir fällen unsere Entscheidung unter Zuhilfenahme einer bestimmten Heuristik, die in ihren einzelnen Schritten intersubjektiv nachvollziehbar ist. Der operationale Charakter unseres Lösungswegs verhindert jedoch nicht, daB verschiedene Analysatoren zu unterschiedlichen Ergebnissen gelangen können, wenn sie uberprüfen sollen, ob die Paraphrase einer gegebenen lexikographischen Definition der von uns aufgestellten inhaltlichen Bedingung genügt.

Die Konstruktion und Durchfürung unseres Tests macht es erforderlich, bestimmte lexikographische Definitionen des Wörterbuchs von OZEGOV neben einer Expandierung einer zusätzlichen Tilgung zu unterziehen. Der Grund, aus dem wir in bestimmten Fällen ganze Semkomplexe aus den Bedeutungsexplikationen tilgen mussen, liegt in der geringen Reglementiertheit, die die Definitionssprache des Wörterbuchs von OZEGOV aufweist.

Wir wollen diese Eigenschaft der OZZEGOVschen Definitionssprache anhand der Bedeutungsexplikationen von udarjat und udar, die wir auch in unser Experiment miteinbeziehen, illustrieren. OŽEGOV definiert diese Lexeme (in einer ihrer Bedeutungen) wie folgt:

(32) $\underline{\text { udarjat' }}=$ 'Nanosit' udar komu-n., proizvodit' udar obo čto-n.' (OZ̈EGOV 1973, 758)

(33) $\underline{\text { udar }}=$ 'Rezkij, sil'nyj tolčok, prikosnovenie $k$ komul čemu-n. s siloj' (OŽEGOV 1973, ebd.)

Betrachten wir die Definitionen (32) und (33) unter dem Aspekt der Bedeutungsbeziehung, die das syntaktische Mittel Komma zwischen den rechts und den links von ihm stehenden Bedeutungskomponenten stiftet, so fällt auf, daB es sich in (32) um die Sinnrelation der Synonymie handelt. Im Wörterbuch von OZZEGOV werden sowohl rezkij als auch sil'nyj (in 
der hier betrachteten Bedeutung) über den Semkomplex 'bol'soj po size' expliziert:

(34) $\underline{r e z k i j}=$ 'Projavljajuščijsja s bol'šoj siloj' (OZEGOV 1973, 622)

(35) $\underline{s i z ' n y j}=$ 'Značitel'nyj po velečine, stepeni' (OZEGOV 1973, 660)

(36) $\underline{\text { značitel'nyj }}=$ 'Bol'šj po razmeru, sile' (OZ̈EGOV 1973, 315)

In (33) stiftet das Komma folglich eine Synonymierelation zwischen 'rezkij' und 'siz'nyj'. Es wird jedoch zusätzlich noch attributiv in der semantischen Funktion eines Modifikations- oder Spezialisierungsverknüpfers zwischen '...tolčok' und 'prikosnovenie...' verwendet (vgl. S. $162 \mathrm{ff.}$ ).43 Die geringe Reglementiertheit der Definitionssprache des Wörterbuchs von OZZEGOV zeigt sich darin, daB mit Hilfe desselben syntaktischen Mittels verschiedene Bedeutungsbeziehungen zwischen Teilen von lexikographischen Definitionen signalisiert werden.

Direkte Konsequenzen für die Durchfïhrung und Konstruktion unseres Tests ergeben sich aus diesem Umstand in all den Fällen, wo lexikographische Definitionen des Wörterbuchs von OZZGOV aufgrund der mangelnden Reglementiertheit seiner Definitionssprache redundante Bedeutungskomponenten enthalten. Wir gehen nämlich von der Annahme aus, daB redundante Bedeutungsexplikationen einen potentiellen Störfaktor für die Durchführung und Aussagekraft unseres Experimentes darstellen. Die Entscheidung für ein Testverfahren, das Informanten anweist, gegebene lexikographische Definitionen (in einer bestimmten Zeitspanne) einer Reduktionsparaphrasierung zu unterziehen, stellt uns vor die Notwendigkeit, das Testmaterial möglichst so zu konstruieren, daB die Aufmerksamkeit der Versuchspersonen nicht unnötig abgelenkt wird. Wir vermuten daher, daB die Fähigkeit der Informanten, Reduktionsparaphrasierungen durchzuführen, sinken wird, wenn in den Bedeutungsexplikationen, die wir in unseren Versuch einbringen, redundante Bedeutungskomponenten nicht getilgt 
werden. Die Notwendigkeit, redundante Seme zu dekodieren, durfte sich kaum positiv auf die Bemühungen der Testpersonen auswirken, die Versuchsinstruktionen zu vollziehen, sondern sie eher verwirren. Aus diesen Gründen eliminieren wir bei der Aufarbeitung des Testmaterials aus den Explikationen (32) und (33) die redundanten Seme 'nanosit' udar komu-n.' bzw. 'siz'nyj', bevor wir (32) durch (33) expandieren (vgl. S. 207 f.). 44

Während die Semredundanz, die bestimmte Bedeutungsexplikationen des wörterbuchs von OZEGOV enthalten, auf die geringe Reglementiertheit von dessen Definitionssprache zuruickzufüren ist, haben wir es in einigen der lexikographischen Definitionen, die wir in unser Experiment einbringen, mit einem anderen Typ von (Sem)Redundanz zu tun, der in der Konstruktion unseres Testmaterials begründet ist und sich sowohl in den Bedeutungsexplikationen des EKW als auch in denen des Wörterbuchs von OZ̈EGOV bemerkbar macht.

Wir wollen, wie bereits erwähnt (vgl. S. $143 \mathrm{f}$.$) , unseren$ Informanten die Bedeutungsexplikationen in einer form präsentieren, in der die Stellen der Kasusrollen (semantischen Valenzen) bzw. bestimmter syntaktischer Valenzen, die in der jeweils zu testenden lexikographischen Definition angesetzt werden bzw. anzusetzen sind, durch konkrete lexikalische Auffüllungen eingenommen werden. Wir halten diese Maßnahme für erforderlich, um die sprachliche Vorstellungskraft unserer Versuchspersonen zu mobilisieren und ihre Fähigkeit zu fördern, eine gegebene, immer noch relativ abstrakte Bedeutungsexplikation einer Reduktionsparaphrasierung zu unterziehen.

Neben der Notwendigkeit, für unseren Test annähernde Homogenität der Versuchspersonen bezüglich ihrer sprachlichen Vorstellungskraft zu erreichen, ist unser Vorgehen auch durch die lexikographische Praxis der gängigen russischen Definitionswörterbücher abgesichert. In der Regel enthalten deren wörterbuchartikel einen direkt auf die lexikographische Definition folgenden Illustrationsteil, in dem einige usuelle Wortverbindungen mit dem betreffenden Stichwort 
angefuhrt werden. So nennt beispielsweise das Wörterbuch von OŽEGOV im Wörterbuchartikel von učit'sja (für eine der Bedeutungen des Stichworts) die Wortverbindung

$$
\text { učit'sja matematike (OŽEGOV 1973, 777). }
$$

Das BAS vermerkt in Anlehnung an eine literarische Quelle den Satz

(38) Starucha moja, gramote srodu ne učilas'.

(BAS 1969, Bd. 16, 1164)

Ein sehr ausfiuhlicher Illustrationsteil findet sich auch in den Wörterbuchartikeln des EKW (vgl. S. 72).

Ein Vergleich der lexikographischen Definitionen des EKW und des Wörterbuchs von OZZEGOV hinsichtlich ihrer Valenzstruktur läßt auf Anhieb Unterschiede erkennen, in denen die lexikographischen Auswirkungen deutlich werden, die sich ihrerseits aus den unterschiedlichen lexikologischen Fundierungen der beiden Wörterbijcher ergeben.

Ein lexikologisches Prinzip im Wörterbuchkonzept der Konstrukteure des MST besteht darin, zwischen der lexikalischen Bedeutung, die einem Stichwort zugewiesen wird, und den Restriktionen seiner semantischen, lexikalischen und morphosyntaktischen. Kombinierbarkeit bezüglich der ihm zugeschriebenen Bedeutung streng zu unterscheiden. Die lexikographische Beschreibung durch das EKW folgt auch weitgehend diesem Prinzip. So enthält jeder bisher erstellte Wörterbuchartikel eine Zone, in der die Explikation der Bedeutung des Stichworts erfolgt, und eine weitere Zone, in der mit Hilfe des Rektionsmodells dessen kombinatorische Eigenschaften beschrieben werden. Dem Rektionsmodell fällt dabei die Aufgabe zu, den in der Bedeutungsexplikation angesetzten Kasusrollen (semantischen Valenzen) syntaktische Valenzen zuzuordnen ( $v g l$. S. $115 \mathrm{ff.}$ ).

Das Prinzip des EKW, die lexikalische Bedeutung und die Kombinierbarkeit des stichworts getrennt zu beschreiben, wurde von uns bereits besprochen ( $v g l$. S. $131 \mathrm{ff.}$ ). In diesem Zusammenhang haben wir einen gewissen Widerspruch konstatiert, in den sich die Konstrukteure des MST bei der semantischen 
Beschreibung begeben. Dieser Widerspruch besteht darin, daB sie einerseits behaupten, in all den zahlreichen Fällen, in denen eine semantische Eigenschaft eines Lexems mit dem gleichen MaB an Vollständigkeit und Widerspruchsfreiheit sowohl als Teil seiner lexikalischen Bedeutung als auch als Teil seines kombinatorischen Potentials aufgefaßt werden kann, aufgrund von Erwägungen zur Einfachheit der Beschreibung den "kombinatorischen" Lösungsweg dem "semantischen" vorzuziehen, während sie andererseits in bestimmte lexikographische Definitionen Bedeutungskomponenten aufnehmen, die eigentlich in Form von Restriktionen der Kombinierbarkeit des Stichworts aufgeführt werden müBten (vgl. S. $137 \mathrm{f.} .^{45}$ Besetzen wir nun, um unser Testmaterial für die geplante Informantenbefragung aufzubereiten, die in den Bedeutungsexplikationen des EKW vorgesehenen Variablen der Kasusrollen mit Wortformen konkreter Lexeme, so ergeben sich in solchen Fällen zwangsläufig Redundanzen. Eine entsprechende lexikalische Auffiillung beispielsweise der lexikographischen Definition (39) von udarjat' mit Wortformen der Lexeme učitel', ukazka und stol, aus der, wie wir bereits dargelegt haben, die Seme 'kompaktnyj predmet' und 'predmet' eigentlich eliminiert und in Kombinationsrestriktionen ubersetzt werden müBten, ergibt die partiell redundante Bedeutungsexplikation (40):

(39) $X \underline{\text { udarjaet }}$ po $Y-u \quad z-o m=' X$ rezko $i$ kratkovremenno privodit kompaktnyj predmet 2 $v$ kontakt 8 predmetom $Y . '$ (APRESJAN 1974b, 108)

(40) Učitel' udarjaet po stolu ukazkoj = 'Učitel' rezko i kratkovremenno privodit kompaktnyj predmet ukcizku $v$ kontakt o predmetom stolom.'

Da wir davon ausgehen, daB redundant kodierte lexikalische Information in den zu paraphrasierenden Bedeutungsexplikationen das sprachliche Vorstellungsvermögen unserer Versuchspersonen negativ beeinflussen könnte (s.o.), sind wir gezwun- 
gen, Redundanzen des oben genannten Typs bei der Konstruktion des Testmaterials zu vermeiden. Für die von uns gewählte Art der Aufbereitung der lexikographischen Definitionen des EKW, die wir in unseren Test einbringen wollen, ist es im Grunde genommen unerheblich, ob eine bestimmte Information in der Bedeutungsexplikation oder in der Beschreibung der Kombinierbarkeit eines Stichworts kodiert werden muB. In all den Fällen, wo die lexikalische Auffüllung von Variablen für Kasusrollen zu wie auch immer motivierten Redundanzen fuhrt, werden wir daher die erforderlichen Tilgungen vornehmen. Im Fall der Bedeutungsexplikation (39) von udarjat' eliminieren wir deshalb die Seme 'kompaktnyj predmet' und 'predmet', um Redundanzen bei der oben genannten lexikalischen Auffüllung der vorgesehenen Kasusrollen zu umgehen; vgl. die entsprechend modifizierte Bedeutungsexplikation (41):

(41) Učitel' udarjaet po stolu ukazkoj = 'Učitel' rezko $i$ kratkovremenno privodit ukazku v kontakt so stolom.

Im Gegensatz zum EKW fehlt im Wörterbuch von OZEGOV, wie auch in anderen Definitionswörterbüchern des Russischen, eine durchgängige und einheitliche Beschreibung der kombinatorischen Eigenschaften der Lexeme. Der Grund für diesen Mangel liegt darin, $d a B$ in der lexikographischen Beschreibung des russischen Wortschatzes nicht zwischen der lexikalischen Bedeutung, die man einem Stichwort zuweist, und den Restriktionen seiner semantischen, lexikalischen und morpho-syntaktischen Kombinierbarkeit mit anderen Lexemen bezijglich dieser Bedeutung unterschieden wird. Verantwortlich für diese Unzulänglichkeiten ist die unzureichende linguistisch-lexikologische Fundierung des Wörterbuchs von OZEGOV. Anhand der Beschreibung der morpho-syntaktischen Kombinierbarkeit und Wortbedeutung, die OZEGOV für arestovyvat', zavidovat' und učit'sja gibt, wollen wir das inkonsequente Vorgehen des Wörterbuchs illustrieren:

(42) arestovyvat' (kogo, čto) = 'Podvergat' kogo-n. arestu 


$$
\begin{aligned}
& \text { ili nakladyvat' arest na } \\
& \text { čto-n.' (OĽEGOV 1973, 30) }
\end{aligned}
$$

(43) zavidovat' (komu, ̌̌emu) = 'Ispytyvat' čuvstvo zavisti

k komu/čemu-n.'

(OZEGOV 1973, 184)

(44) ǔcit'sja (čemu ili neopr.) = 'Usvaivat' kakie-n. znanija, navyki' (OŽEGOV 1973, 777)

Das Wörterbuch von OZEGOV bedient sich sogenannter Kasusfragen (padežnye voprosy, OZEGOV 1973, 16), um Angaben zur morpho-syntaktischen Kombinierbarkeit des Stichworts zu machen. Beurteilen wir die lexikographischen Definitionen (42) - (44) aus der Sicht des valenzgrammatischen Ansatzes von HELBIG/ SCHENKEL (HELBIG/SCHENKEL 1973), so müssen wir feststellen, daß sie die syntaktische Valenzstruktur der Lexeme nur unzureichend abbilden.

In (42) wird mit Hilfe der Kasusfrage kogo/čto eine Leerstelle für die zweite syntaktische Valenz ausgewiesen, während die entsprechende Leerstelle für die dritte syntaktische Valenz nicht angesetzt wird. Ausschlaggebend für diese Valenzbeschreibung von arestovyvat' mag der Umstand sein, daB die zweite syntaktische Valenz im Sinne von HELBIG/SCHENKEL obligatorisch, die dritte syntaktische Valenz fakultativ ist (HELBIG/SCHENKEL 1973, 31-40).

Analog $z u(42)$ ist in der lexikographischen Definition von zavidovat, (43) für die zweite obligatorische syntaktische Valenz durch die Kasusfrage komu/čemu eine Leerstelle ausgewiesen, für die dritte fakultative syntaktische Valenz jedoch nicht. Im Unterschied zu (42) und (43), die nur Leerstellen für obligatorische syntaktische Valenzen enthalten, wird in der lexikographischen Definition von učit'sja (44) mit Hilfe der Kasusfrage čemu bzw. der Angabe neopr. (= neopredelënnaja forma) eine Leerstelle für eine fakultative syntaktische Valenz ausgewiesen.

AuBerdem fehlt in allen drei Bedeutungsexplikationen, wie übrigens in sämtlichen Wörterbuchartikeln des Lexikons, die Leerstelle für die syntaktische Subjektsvalenz. Diese Tat- 
sache muB nicht unbedingt als ein lexikographischer Mangel des Wörterbuchs angesehen werden. Die spezifischen Bedingungen, die das Russische für die Weglabbarkeit der lexikalischen Realisierungen dieser Valenz in den sogenannten unpersönlichen Konstruktionen bietet, sowie die geringen Variationsmöglichkeiten, denen die morpho-syntaktische Realisierung der syntaktischen Subjektsvalenz unterworfen ist, können ein derartiges Vorgehen in der lexikographischen Praxis rechtfertigen.

Aus lexikographischer Sicht nicht zu rechtfertigen ist dagegen das inkonsequente Vorgehen, mit der die morpho-syntaktische Kombinierbarkeit der Lexeme im Hinblick auf die übrigen Valenzen beschrieben wird. Das Wörterbuch von OZ̈EGOV ist nicht in der Lage, potentiellen Benutzern, die sich beispielsweise uber die Rektion eines bestimmten Stichworts informieren möchten, hinreichende Angaben zu machen. Die Tatsache, daB in seinen lexikographischen Definitionen häufig Leerstellen fir fakultative syntaktische valenzen fehlen, schmälert den lexikographischen Nutzwert des Wörterbuchs auBerordentlich, da es in diesen Fällen auch keine Hinweise auf Realisierungsmöglichkeiten solcher syntaktischen Valenzen gibt, die bei einem bestimmten Stichwort idiomatisch durch komplexe lexikalisch-grammatische Ausdrucksmittel realisiert werden können und gerade deswegen für potentielle Benutzer von grobem Interesse sein diuften; vgl. z.8. die Wortverbindungen arestovyvat' kogo-n. po obvineniju $v$ sanť̌e/za šantă und zavidovat' komu-n. za chorošie ocenkil iz-za chorosich ocenok.

Die unzureichende Trennung, die das Wörterbuch von OZ̈EGOV zwischen der Beschreibung der Wortbedeutung und den Angaben zur Kombinierbarkeit des Stichworts mit anderen Lexemen vornimmt, zeigt sich darin, daB die syntaktischen Valenzen, die dem Stichwort zugewiesen werden, in den lexikographischen Definitionen häufig als Leerstellen für Kasusrollen ausgewiesen werden. Aufgrund der luckenhaften Spezifizierung der syntaktischen Valenzen eines Stichworts kommt es daher oft vor, daB die OZZEGOVschen Bedeutungsexplikationen nicht allen Aktanten, die in der durch das Stichwort bezeichneten Situation anzusetzen sind, eine Leepustantein - $7 p p 954792764$ 
räumen; vgl. das Fehlen der Kasusrolle 'Motiv' in (43). 46

Andererseits zeichnen sich zahlreiche lexikographische Definitonen des Wörterbuchs von OZ̈EGOV dadurch aus, daB in ihnen jegliche Leerstellen für Kasusrollen fehlen, während Angaben zur morpho-syntaktischen Kombinierbarkeit des betreffenden Stichworts gemacht werden (vgl. S. $131 \mathrm{f.}$ ).

Die Bedeutungsexplikationen des Wörterbuchs von OŽEGOV geben auch sehr häufig Informationen zu Restriktionen, denen die semantische Kombinierbarkeit des Stichworts mit Wortformen anderer Lexeme unterliegt. In der Definition von zavidovat' (43) weist die Angabe $k$ komu/čemu-n. darauf $h i n$, daB die Lexeme, deren Wortformen an die Stelle der zweiten syntaktischen Valenz von zavidovat, treten können, belebte und/oder unbelebte Referenten bezeichnen können.

Die Spezifizierungen 'znanija, navyki' in der Explikation von učit'sja (44) geben an, daß die entsprechende Valenzstelle nur von Wortformen solcher Lexeme eingenommen werden kann, bei denen es sich um Bezeichnungen für Wissensgebiete und/oder Fertigkeiten ('znanija, navyki') handelt.

Die mangelnde Trennung zwischen der Bedeutungsexplikation und den Angaben zur Kombinierbarkeit des Stichworts, die charakteristisch für viele Artikel des Wörterbuchs von OZ̈EGOV ist, macht es erforderlich, um zu vermeiden, daB in (43) und (44) lexikalische Informationen redundant kodiert werden, die oben genannten Bedeutungskomponenten zu tilgen, wenn die Kasusrolle 'obj' in (43) und 'Content' in (44) be ispielsweise durch Wortformen der Lexeme brat bzw. matematika realisiert werden.

Konsequenzen für die Konstruktion unseres Testmaterials ergeben sich ebenfalls aus der unvollständigen Beschreibung der Valenz- und der Rollenstruktur von Lexemen im Wörterbuch von 0ZZEGOV. Wir düfen nur diejenigen Stellen für Kasusrollen mit Wortformen bestimmter Lexeme besetzen, die in den von uns expandierten lexikographischen Definitionen des Wörterbuchs von OZEGOV vorgesehen sind. Dieser Umstand führt dazu, daß die Bedeutungsexplikationen des Wörterbuchs von 
OZ̈EGOV und des EKW, die wir den Versuchspersonen zur Reduktionsparaphrasierung vorlegen, sich in der Anzahl der konkreten lexikalischen Auffüllungen ihrer Stellen für Kasusrollen unterscheiden können. Dies ist beispielsweise bei den Bedeutungsexplikationen der Fall, die das EKW und nach entsprechender Expandierung das Wörterbuch von OZ̈EGOV für udarjat' anfihren; vgl. die lexikographischen Definitionen (45) und (46):

(45) 'Učitel' rezko i kratkovremenno privodit ukazku $v$ kontakt so stolom.'

(46) 'Proizvodja rezkij tolčok, učitel' prikasaetsja s siloj k stolu.'

Wir sind gezwungen, bei der Aufarbeitung des Testmaterials unser Konstruktionsprinzip, nur diejenigen Kasusrollen in den von uns expandierten OZ̈EGOVschen lexikographischen Definitionen mit Wortformen bestimmter Lexeme zu besetzen, die in diesen ausgewiesen sind, in einigen fällen aufzugeben. Diese Notwendigkeit ergibt sich immer dann, wenn die Bedeutungsexplikationen des Wörterbuchs von OŽEGOV, die wir in unser Experiment miteinbeziehen, bestimmten Kasusrollen, deren syntaktische Realisierung bei einem gegebenen Stichwort obligatorisch ist, keine Leerstellen einräumen bzw. Leerstellen für Kasusrollen überhaupt nicht enthalten.

Die lexikographische Definition (47), die OŽEGOV beispielsweise dem Verb provocirovat' (in einer seiner Bedeutungen) zuweist, sieht für die Kasusrolle ' $O b j$ ' keine Leerstelle vor, obwohl ihre Realisierung bei diesem Stichwort obligatorisch ist:

$$
\begin{aligned}
\text { provocirovat' = } & \text { 'Vyzyvat' na kakie-n. dejstvija putëm } \\
& \text { provokacii' (OZZEGOV 1973, 558) }
\end{aligned}
$$

Wir müssen daher in (47) für die Kasusrolle 'Obj' eine Leerstelle ausweisen, um sie neben den beiden anderen Stellen für die Kasusrolle 'sub' und 'Dest' mit Wortformen bestimmter Lexeme wie beispielsweise gusar, sopernik und draka besetzen zu können. Führen wir die in (47) erforderlichen Tilgungen redundanter Bedeutungskomponenten durch, erhalten 
wir die lexikographische Definition

(48) 'Gusar vyzyvaet sopernika na draku putem provokacii.'

Um den Versuchspersonen die lexikographische Definition von provocirovat' zur Reduktionsparaphrasierung vorlegen zu können, müssen wir (48) nur noch expandieren, indem wir z.B. die Bedeutungskomponente 'provokaoii' entfalten (vgl. S. $203 \mathrm{f}$.)

Stellvertretend für die zahlreichen lexikographischen Definitionen im wörterbuch von OZEGOV, die überhaupt keine Leerstellen für Kaususrollen vorsehen, wollen wir die Explikation (49) von issledovat' (in einer seiner Bedeutungen) anführen:

(49) $\underline{\text { issledovat' }}$ = 'Podvergat' naučnomu izučeniju' (OZEGOV 1973, 236).

Wir müssen in (49) je eine Stelle für die Kasusrolle 'Sub' und ' $O b j$ ' ansetzen, da deren Realisierung bei issledovat' obligatorisch ist. Erst dann können wir diese mit Wortformen beispielsweise der Lexeme biolog und volk besetzen:

(50) 'Biolog podvergaet volkov naučnomu izučeniju.'

Entfalten wir in (50) z.B. die Bedeutungskomponente 'naučnomu', können wir den Versuchspersonen die lexikographische Definition von issledovat' zur Reduktionsparaphrasierung vorlegen ( $v g l$. S. $195 \mathrm{ff.}$ ).

Der Status der Definitionssprache des Wörterbuchs von OZEGOV zwingt uns, bei der Aufarbeitung des Testmaterials im Hinblick auf die OZEGOVschen Bedeutungsexplikationen, die wir in unser Experiment aufnehmen, ein weiteres Konstruktionsprinzip zu beachten. Wir müssen bei der Expandierung der Bedeutungsexplikationen des Wörterbuchs von OZEGOV stets dem Umstand Rechnung tragen, daB es sich bei seiner Definitionssprache um eine natürliche Sprache (= Russisch) handelt. M.a.W., wir sind gezwungen, peinlich darauf zu achten, daB wir zumindest die grammatische Wohlgeformtheit der lexikographischen Definitionen des Wörterbuchs von OZEGOV nicht verletzen. Es ist daher in einigen Fällen erforderlich, bestimmte Bedeutungskomponenten in OZEGOVschen lexikographi- 
schen Definitionen nach ihrer vorherigen Expandierung zu verändern, um die grammatische Wohlgeformtheit der Bedeutungsexplikationen zu erhalten. Da sich unsere Modifikationen ausschlieblich auf die kombinatorischen Eigenschaften bestimmter (russischer) Wörter, denen in den Explikationen Semstatus zukommt, beziehen düren, bleibt die Gesamtbedeutung der jeweiligen lexikographischen Definition selbstverständlich unverändert.

Expandieren wir beispielsweise die Explikation, die OŽEGOV fur nakazyvat' gibt

(51) $\underline{\text { nakazyvat' }}=$ 'Podvergat' nakazaniju' (OŽEGOV 1973, 347), indem wir das Sem 'nakazaniju' durch seine lexikographische Definition

(52) $\underline{\text { nakazanie }}=$ 'Mera vozdejstvija protiv soveršivšego prestuplenie, prostupok' (OŽEGOV 1973 , ebd.)

ersetzen, müssen wir Modifikationen vornehmen, die sich aus Beschränkungen ergeben, denen die lexikalische Kombinierbarkeit der Bedeutungskomponente 'mera' unterliegt. Um zu vermeiden, daB als Folge der Substitution von 'nakazaniju' in (51) durch die Bedeutungsexplikation (52) die nichtwohlgeformte Wortverbindung 'podvergat' mere' entsteht, ist in (51) das Verb 'podvergat' 'gegen das Verb 'prinimjat' ' auszutauschen (vgl. auch S. $198 \mathrm{f}$.). Als Ergebnis unserer Veränderungen erhalten wir die nunmehr grammatisch wohlgeformte lexikographische Definition

(53) $\underline{\text { nakazyvat' }}=$ 'Prinimat' meru vozdejstvija protiv soveršivšego prestuplenie, prostupok'

Da es sich bei der SemS des EWK um eine Kunstsprache handelt, der die Konstrukteure des MST U.a. aufgrund der Eigenschaft, daß in ihr bestimmte syntaktische und lexikalische Regeln der russischen Grammatik nicht beachtet werden (vgl. S. 140) eine effektive Verwendbarkeit für lexikographische Zwecke zusprechen, machen wir uns bei den Bedeutungsexplikationen des $E K W$, die wir in unseren Versuchen miteinbeziehen, über Fragen 
ihrer Wohlgeformtheit keine Gedanken. M.a.W., wir gehen in unserem Experiment von der ungeprüften Annahme aus, daB all die Auswirkungen der Reglementiertheit der SemS des EKW, die sich in Abweichungen von grammatisch-lexikalischen Normen des Russischen manifestieren, die Verstehbarkeit der Sems positiv beeinflussen. Um die Fähigkeit unserer Informanten, Reduktionsparaphrasierungen auszuführen, nicht unnötig durch komplizierte Erläuterungen zu bestimmten Bedeutungskomponenten der Sems vor Beginn des Experiments zu beeinträchtigen (vg). S. 47), ersetzen wir in den lexikographischen Definitionen des EKW lediglich das "Kunstwort" 'kauzirovat" durch 'pričinjat' '. Hierzu sind wir berechtigt, da die Konstrukteure des MST in einigen Bedeutungsexplikationen genauso verfahren (vgl. die lexikographische Definition von nakazyvat'), und sich eventuell aus dieser Substitution ergebende Verstöße gegen grammatisch-lexikalische Normen mit dem oben genannten Reglementierungsprinzip der Sems kompatibel sind. Nachdem wir die Prinzipien besprochen haben, von denen wir uns bei der Konstruktion unseres Testmaterials leiten lassen wollen, können wir jetzt dazu übergehen, die lexikographischen Definitionen der Lexeme, die Gegenstand unseres Experiments sein sollen, gemäß diesen Prinzipien zu bearbeiten und anzuordnen.

\section{3 Auswahl des Testmaterials}

Wir haben für unseren Versuch die lexikographischen Definitionen von 17 verschiedenen Stichwörtern ausgewählt, die im Wörterbuch von OŽEGOV und im EKW (in einer ihrer Bedeutungen) expliziert werden. Bei diesen Stichwörtern handelt es sich um 13 Verben und 4 Adjektive. Die Anzahl von 17 Stichwörtern ergibt sich aufgrund der Beschränkungen, denen die Auswahl unseres Testmaterials unterworfen ist. Ein hohes MaB an Restriktion wird, wie bereits erwähnt (vgl. S. $168 \mathrm{ff}$.$) , den$ uns zur Verfügung stehenden Daten durch die Zirkularität auferlegt, die sich in den lexikographischen Definitionen des Wörterbuchs von OŽEGOV einstellt, wenn diese auch nur ein 
einziges Mal expandiert werden.

Weitere Beschränkungen resultieren aus der unterschiedlichen Behandlung polysemer Stichwörter im Wörterbuch von OZEGOV und im EKW. In den Wörterbuchartikeln bestimmter polysemer Lexeme im Wörterbuch von OZEGOV werden die verschiedenen Bedeutungen, die dem Stichwort zugeschrieben werden, durch lexikographische Definitionen expliziert, von denen keine einzige der bzw. den Bedeutungen entspricht, die das EKW für das betreffende Stichwort expliziert. Anhand der lexikographischen Definition von beispielsweise opredeljat' im Wörterbuch von OŽEGOV und im EKW IäBt sich das unterschiedliche Vorgehen beider Wörterbücher illustrieren. Für eine der Bedeutungen des Verbs opredeljat, gibt das EKW die folgende Bedeutungsexplikation:

(54) B opredeljaet $X$ (naprimer, Vospitanie opredeljaet nravstvennyj oblik čeloveka) =

' $X$ zavisit ot $B$, $i$ zavisimost' $x-a$ ot $B-a$ bol'še, čem ot drugich vozmožnych faktorov.' (APRESJAN 1974b, 108-109)

Dieser lexikographischen Definition des polysemen Stichworts opredeljat, entspricht keine der Bedeutungen, die das wörterbuch von OZEGOV dem Verb zuweist und expliziert. OZEGOV definiert wie folgt:

(55) opredeljat' $=1$. 's točnost'ju vyjasnjat', ustanavlivat' ': opredeljat' bolezn'.

2. 'Raskryvat' slovami soderžanie čego-n. '; opredeljat' novoe naučnoe ponjatie.

3. To že, čto obuslavlivat' (vo 2 znắ.).

4. 'Naznačat', ustraivat' na kakuju-n. dolžnost' ili v kakoe-n. učebnoe zavedenie' (ustar. i prost.); opredeljat' na službu. (OŽEGOV 1973, 416)

(56) $\underline{\text { obuslavlivat' }}=2$. 'Javljat'sja pričinoj čego-n., vyzyvat' čto-n.'; Planomernyj trud obuslavlivaet uspech dela. (OZEGOV 1973, 399) 
Schließlich sei noch darauf hingewiesen, daß von den zahlreichen Wörterbuchartikeln, die die Konstrukteure des MST und deren Mitarbeiter in der Serie "Predvaritel'nye publikacii problemnoj gruppy po éksperimental'noj i prikladnoj lingvistike Instituta Russkogo Jazyka AN SSSR" veröffentlicht haben, uns nur die Ausgaben Nr. 37, 38 und 62 der "Materialy k tolkovo-kombinatornomu slovarju russkogo jazyka" zur Verfügung standen. Wir konnten uns daher nur auf insgesamt ca. 50 verschiedene lexikographische Definitionen des EKW stiutzen, die in den uns zugänglichen Publikationen der Konstrukteure des MST angeführt werden.

Bei den 17 Lexemen, deren lexikographische Definitionen das Material unseres Experiments bilden, handelt es sich um die folgenden (in kyrillisch alphabetischer Reihenfolge genannten) Verben und Adjektive:

arestovyvat', drobit', zaviset', nabljudat', nakazyvat', predlagat', prenebregat', provocirovat', razbivat', ubeždat' udarjat', učityvat', učit'sja; ostorožnyj, pročnyj, spravedlivyj, jasnyj.

Wir wollen nun die lexikographischen Definitionen der oben genannten Stichwörter, die das Wörterbuch von OZEGOV und das EKW ihnen (in je einer ihrer Bedeutungen) zuweisen, nacheinander betrachten und gemäß unseren Oberlegungen zur Konstruktion des Testmaterials anordnen. 


\section{Konstruktion des Testmaterials}

1. arestovyvat'

a) arestovyvat' (OZEGOV)

(1) arestovyvat' = 'Podvergat' kogo-n. arestu ili nakladyvat' arest na čto-n.' (OZEGOV 1973, 30)

In (1) muß der Semkomplex 'ili nakladyvat' arest na čto-n.' getilgt werden, da OZEGOV innerhalb derselben lexikographischen Definition zwei verschiedene Bedeutungen des polysemen Lexems arestovyvat, expliziert: ${ }^{47}$

(2) 'Podvergat'kogo-n. arestu'

Wir expandieren (2) in (4), indem wir die Bedeutungskomponente 'arestu' durch

(3) $\underline{\text { arest }}=$ 'Zaključenie pod stražu' (OZEGOV 1973, ebd.) ersetzen:

(4) 'Podvergat' kogo-n. zaključeniju pod stražu'

Die in (4) angesetzten bzw. anzusetzenden Kasusrollen 'sub' und ' $O b j '$ ', deren syntaktische Realisierung obligatorisch ist, besetzen wir mit Wortformen der Lexeme policija und Pëtr:

(5) 'Policija podvergaet Petra zaključeniju pod stražu.'

Wir legen den Versuchspersonen die lexikographische Definition von arestovyvat, in der unter (5) angeführten Form zur Reduktionsparaphrasierung vor.

b) arestovyvat' 1 (EKW)

(1) $X$ arestovyvaet $y-a z a z=$ 'oficial'nye organy $X v$ sootvetstuii s predvaritel'nym rasporjaženiem $l_{i-}$ sajut $y$-a suobody za ego 


\section{dejstvija 2.' (MEL'CUK $1973,116)$}

Wir besetzen die in (1) ausgewiesenen Kasusrollen ' $X$ ' ('sub'), ' $y$ ' ('obj') und ' $Z$ ' ('Motiv') mit Wortformeln der Lexeme policija, Pëtr und vorovstvo. Außerdem tilgen wir die Bedeutungskomponenten 'oficial'nye organy' und 'dejstvija', um Redundanzen zu vermeiden:

(2) 'Policija v sootvetstvii s predvaritel'nym rasporjaženiem lisăet Petra suobody za vorovstvo.

Wir legen den Versuchspersonen die lexikographische Definition von arestovyvat' in der unter (2) angeführten Form zur Reduktionsparaphrasierung vor.

2. drobit,

a) drobit' (OZEGOV)

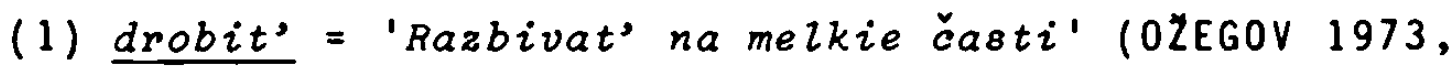
165)

Wir expandieren (1), indem wir die Bedeutungskomponente 'razbivat"' durch ihre Explikation ersetzen. Wir gelangen erst dann zu einer Substitution von 'razbivat' ' durch andere Bedeutungskomponenten, die den von uns aufgestellten Bedingungen für eine Expandierung einer lexikographischen Definition genügt, nachdem wir im Wörterbuch mehreren Verweisen gefolgt sind:

(2) $\underline{\text { razbivat' }}=$ Sm.bit' (OZEGOV 1973, 589)

(3) $\underline{\text { bit' }}$ = 'Lomat', razdrabljat' rasskalyvat' ' (OZEGOV 1973,48 )

(4) $\underline{\text { lomat' }}$ = 'Sgibaja ili udarjaja s siloj, razdeljat' nadvoe, na kuski, na časti, otdeljat' časti čego-n.' (OZEGOV 1973, 302)

In (4) tilgen wir die Bedeutungskomponenten 'nadvoe, na $k u s k i$, na časti' und 'otdeljat' časti čego-n.' um zu vermeiden, daß bei ihrer Einbettung in (1) Semredundanz ent- 
steht:

(5) 'sgibaja ili udarjaja s siloj, razdeljat' na melkie
casti'

Wir besetzen die in (5) anzusetzenden Kasusrollen 'sub' und ' $O b j$ ', deren syntaktische Realisierung obligatorisch ist, mit Wortformen der Lexeme Pëtr und $l^{\prime}$ dina:

(6) 'Sgibaja ili udarjaja s silojj, Pëtr razdeljaet l'dinu

Wir legen den Versuchspersonen die lexikographische Definition von drobit, in der unter (6) angefiuhten form zur Reduktionsparaphrasierung vor.

b) drobits (EKW)
(1) $A$ drobit $B X$-om = 'A delit tvërdyj i pročnyj $B$ udarom instrumenta $X . '$ (APRESJAN $1974 \mathrm{~b}$, 108)

Wir besetzen die in (1) ausgewiesenen Kasusrollen ' $A$ ' ('sub'), 'B' ('obj') und ' $X$ ' ('Instr') mit Wortformen der Lexeme Pëtr, $l^{\prime}$ dina und molot und tilgen den Semkomplex 'tvërdy $i$ pročnyj', um Redundanz zu vermeiden. AuBerdem streichen wir in (1) die Bedeutungskomponente 'instrumenta'.

Bei diesem Sem handelt es sich nicht um eine Angabe zur semantischen Kombinierbarkeit des Stichworts mit anderen Lexemen bezüglich bestimmter Realisierungsmöglichkeiten des Kasusrolle 'Instr', die inkonsequenterweise in die Bedeutungsbeschreibung von drobit, aufgenommen worden ist, sondern vielmehr analog zur Explikation von rubit' (APRESJAN 1974b, 63) um eine Komponente, die APRESJAN als paradigmatisch für die Bedeutung des Stichworts ansieht. Das Verb drobit, kann nicht ausschlieblich mit Lexemen verknüpft werden, die zur klasse der Bezeichnungen für Instrumente wie beispielsweise topor, molot, me č u.ä. gehören, sondern mit beliebigen Bezeichnungen für Realien, denen in der durch drobit' bezeichneten Situation die Funk- 
tion eines Instruments zugewiesen wird; vgl. beispielsweise die Wortverbindungen drobit' l'dinu kulakom, lokot'ju, doskoj, kam'ju.

Die Streichung des Sems 'instrumenta' müssen wir deshalb vornehmen, weil die Konstrukteure des MST für drobit' bisher noch keine Tilgungsregel entwickelt haben (vgl. S. $136 \mathrm{ff}$.$) . Diese Regel müßte angeben, daß bei Besetzung der$ Variablen ' $X$ ' durch Bezeichnungen für Instrumente die Bedeutungskomponente 'instrumenta' $z u$ tilgen ist, bei ihrer Besetzung durch Bezeichnungen für andere Realien, denen in der durch drobit' bezeichneten Situation nur die Funktion eines Instruments verliehen wird, dieses Sem jedoch in die Angabe 'ispol'zuja $X v$ kačestve instrumenta' umzuformen ist.

Als Ergebnis der oben genannten Modifikationen erhalten wir die Bedeutungsexplikation

(2) 'Pëtr delit l'dinu udarom molota.'

Aus Grïnden der Vergleichbarkeit zwischen der lexikographischen Definition, die das Wörterbuch von OZ̈EGOV für drobit' gibt, und der des EKW könnte erwogen werden, die Prinzipien, nach denen wir das Testmaterial konstruieren wollen, in diesem Fall außer kraft zu setzen und, um eine annähernde Ahnlichkeit $z$ wischen beiden Bedeutungsexplikationen herbeizuführen, das Sem 'udarom' in (2) durch die lexikographische Definition des EKW für udarjat' (vgl.

S. 209) zu ersetzen. Eine entsprechende Expandierung von (2) ergäbe die Bedeutungsexplikation

(3) 'Pëtr delit l'dinu, privodja rezko i kratkovremenno molot v kontakt 8 nej.'

Gegen diese Oberlegungen spricht jedoch die Tatsache, daB unseren Versuchspersonen im Laufe des Experiments auch die lexikographische Definition von udarjat' (EKW) vorgelegt wird. Es ist daher nicht ausgeschlossen, daß die Fähigkeit der Informanten, Reduktionsparaphrasierungen vorzunehmen, durch eine sogenannte homogene Hemmung aufgrund 
teilidentischen und somit nur schwach kontrastierten Testmaterials (JUHÁSZ 1970, 92ff.) negativ beeinfluBt wird. 48 Aus diesem Grund legen wir den Versuchspersonen die lexikographische Definition von drobit, in der unter (2) angefinrten Form zur Reduktionsparaphrasierung vor.

3. zaviset'

a) zavieet" (OZEGOV)

(1) $\underline{\text { zaviset' }}=$ 'Nachodit'sja v zavisimosti' (OŽEGOV 1973, 184)

Wir expandieren (1) in (3), indem wir die Bedeutungskomponente 'zavisimosti' durch

(2) $\underline{\text { zavieimost' }}=$ 'Podčinënnost' drugim, čužoj vole, čužoj vlasti pri otsutstvii samostojatel'nosti' (OZEGOV 1973, ebd.)

ersetzen:

(3) 'Nachodit'sja $v$ podčinënnosti drugim, čužoj vole, čuzoj vlasti pri otsutstvii samostojatel'nosti'

Die in (3) anzusetzenden Kasusrollen 'Sub' und 'Contrag', deren syntaktische Realisierung obligatorisch ist, besetzen wir mit Wortformen der Lexeme brigadir und načal'nik. Die lexikalische Auffüllung von (3) durch diese Lexeme macht es erforderlich, aus (3) die Bedeutungskomponenten 'drugim, čužoj vole, čužoj vlasti' zu streichen, um Semredundanzen auszuschalten:

(4) 'Brigadir nachoditsja $v$ podčinënnosti u načal'nika pri otsutstvii samostojatel'nosti.

Wir legen den Versuchspersonen die lexikographische Definition von zaviset, in der unter (4) angeführten form zur Reduktionsparaphrasierung vor. 
b) zaviset' (EKW)

(1) $\begin{aligned} X \text { zavisit ot } B-a= & \text { B možet kauzirovat' izmenenija } \\ & v \text { svojstvach, sostojanijach ili } \\ & \text { povedenii X-a. ' (APRESJAN 1974b, } \\ & 108 \text { ) }\end{aligned}$

Wir überführen (1) in (2), indem wir die in (1) ausgewiesenen Kasusrollen ' $B$ ' ('Sub') und ' $X$ ' ('obj') mit Wortformen der Lexeme načal' $n i k$ und brigadir besetzen und die Bedeutungskomponente 'kauzirovat' durch 'pričinjat' ' etikettieren ( $\mathrm{vgl}$. S. 187):
(2) 'Načal'nik možet pričinjat' izmenenija v svojstvach, sostojanijach $i l i$ povedenii brigadira.'

Wir legen den Versuchspersonen die lexikographische Definition von zaviset, in der unter (2) angefürten form zur Reduktionsparaphrasierung vor. Dabei erhalten die Informanten die zusätzliche Instruktion (3), die Bedeutungsexplikation (2), die bezüglich der verbgebundenen oberflächensyntaktischen Mitspieler von zaviset, eine Konversionsstruktur aufweist, so umzuformen, daB in der Reduktionsparaphrase der durch eine Wortform von brigadir besetzten Kasusrolle die syntaktische Funktion des Subjekts und der durch eine Wortform von načal'nik besetzten Kasusrolle die syntaktische Funktion eines Präpositionalobjekts zukommt :

(3) Zamečanie:

Upotrebite pri sokraščenii slovo "brigadir" v kačestue podležă̌čego i slovo "načal'nik" v kačestve predložnogo dopolnenija!

4. nabljudat'

a) nabljudat' (OZEGOV)

(1) $\underline{\text { nabljudat' }}=$ 'Izučat', issledovat' ' (OZEGOV 1973, 339) 
Wir tilgen in (1) die Bedeutungskomponente 'izučat'', da sie mit der Bedeutungskomponente 'issledovat' synonym ist. Wir expandieren (1), indem wir das Sem 'isszedovat'" durch seine Explikation

(2) $\underline{\text { issledovat' }}$ = 'Podvergat' naučnomu izučeniju' (OZEGOV 1973, 236)

ersetzen.

Im Fall der lexikographischen Definition von nabljudat' in der oben genannten Bedeutung haben wir es mit dem für das Wörterbuch von OZEGOV seltenen Fall zu tun, daB der Dekompositionsgrad einer Bedeutungsexplikation durch zwei weitere Expandierungen erhöht werden kann. Wir ersetzen in (2) das Sem 'naučnomu' durch

(3) $\underline{\text { naučnyj }}$ = 'Osnovannyj na principach nauki, otvečajuščij trebovanijam nauki' (OZEGOV 1973, 361)

und erhalten die Explikation

(4) 'Podvergat' izučeniju, osnovannomu na principach nauki, otvečajuščemu trebovanijam nauki'

In (4) tilgen wir den redundanten Semkomplex 'otvečajuščemu trebovanijam nauki' und ersetzen das Sem 'nauki' durch

(5) $\underline{\text { nauka }}=$ 'Sistema znanij o zakonomernostjach $v$ razvitii prirody, obščstua i myšlenija, a takže otdel'naja otrasl' takich znanij' (OZEGOV 1973, ebd.)

Als Ergebnis unserer Modifikationen erhalten wir

(6) 'Podvergat' izučeniju, osnovannomu na principach sistemy znanij o zakonomernostjach v razvitii prirody, obščestua $i$ myšlenija, a taǩ̃e otdel'noj otracli takich znanij'

Da die lexikographische Definition (5), die OZEGOV nauka zuweist, zugleich zwei Bedeutungen des Stichworts expli- 
ziert, tilgen wir in (6) den Semkomplex ', a taǩze otdel'noj otrasli takich znanij', weil er die speziellere Bedeutung von nauka beschreibt:

(7) 'Podvergat' izučeniju, osnovannomu na principach sistemy znanij o zakonomernostjach $v$ razvitii prirody, obščestva i myšlenija'

Wir besetzen die in (7) anzusetzenden Kasusrollen 'Sub' und ' $O b j$ ', deren syntaktische Realisierung obligatorisch ist, mit Wortformen der Lexeme biolog und volk. Aufgrund der durch nabljudat' typischerweise bezeichneten auBersprachlichen Situationen nehmen wir auBerdem als gesichertes Weltwissen an, daB bei dieser lexikalischen Auffüllung der Kasusrollen des Stichworts die Seme ' $i$ my:s Lenija' nicht aktualisiert werden, wenn Sprecher entsprechende Sätze mit nabljudat' dekodieren:

(8) 'Biolog podvergaet volkov izučeniju, osnovannomu na principach sistemy znanij o zakonomernostjach $v$ razvitii prirody, objècestva.

Wir legen den Versuchspersonen die lexikographische Definition von nabljudat' in der unter (8) angeführten Form zur Reduktionsparaphrasierung vor.

b) $n a b l j u d a t$, (EKH)

(1) A nabljudaet $X=$ 'A sledit za povedeniem $X-a$ s cel' $j u$ uznat' svojstva $x$-a.' (APRESJAN 1974b, 108)

Wir formen (1) in (2) um, indem wir die ausgewiesenen kasusrollen ' $A$ ' ('sub') und ' $X$ ' ('obj') durch Wortformen der Lexeme biolog und volk sowie durch das (anaphorische) Possessivpronomen ich besetzen:

(2) 'Biolog sledit za povedeniem volkov s cel'ju uznat' ich svojstva. '

In der unter (2) angefiuhten Form legen wir den Versuchspersonen die lexikographische Definition von nabljudat' 
zur Reduktionsparaphrasierung vor.

\section{5. nakazyvat'}

a) nakazyvat' (OŽEGOV)

(1) $\underline{\text { nakazyvat' }}=$ 'Podvergat' nakazaniju' (OŽEGOV 1973, 347 )

Wir expandieren (1) in (3), indem wir die Bedeutungskomponente 'nakazaniju' durch

(2) $\underline{\text { nakazanie }}=$ 'Mera vozdejstvija protiv soveršivšego prestuplenie, prostupok' (OZEGOV 1973, ebd.)

ersetzen:

(3) " 'Podvergat' mere vozdejstvija protiv soveršivšego prestuplenie, prostupok'

Wir besetzen die in (3) anzusetzenden Kasusrollen 'sub' und ' $O b j$ ', deren syntaktische Realisierung obligatorisch ist, mit Wortformen der Lexeme tovarišć Ly̌̌nikov und tovarǐsc Ivanov:

(4) "Tovarǐsc Ly̌̃nikov podvergaet tovarišča Ivanova mere vozdejstvija protiv soveršivšego prestuplenie, prostupok.'

Die Expandierung von (1) in (3) bzw. (4) führt zu einem nichtwohlgeformten Satz, weil in dem Syntagma "podvergat' mere (vozdejstvija)' mit dem Verb 'podvergat' ' ein falsches lexikalisches Ausdrucksmittel für eine syntaktische Verknüpfung ausgewählt worden ist, die den Aktanten, der die Agensrolle in der durch mera (vozdejstvija) bezeichneten Situation spielt, in der Funktion des syntaktischen Subjekts mit dem entsprechenden Aktanten in Objektsrolle in der Funktion des ersten syntaktischen objekts und der Bezeichnung der Situation in der Funktion des zweiten syntaktischen objekts verbindet. Ausgedrijckt in den Termini der MEL'CUKschen lexikalischen Funktionen (LF) ist fur das 
Stichwort mera der Wert der LF Labor ${ }_{12}$, der die lexikalischen Realisierungsmöglichkeiten der oben beschriebenen syntaktischen Verknüpfung angibt, nicht das Verb podvergat'. Da für das Stichwort mera eine LF Labor ${ }_{12}$ im Russischen nicht definiert ist, müssen wir, um die grammatische Wohlgeformtheit von (3) bzw. (4) wiederherzustellen, eine andere syntaktische Verknüpfung zwischen den lexikalischen Auffüllungen der beiden oben genannten Kasusrollen und mera auswählen.

Diese liefert uns die LF Oper $_{1}$, die das Verb angibt, das den ersten Aktanten in der durch mera bezeichneten Situation in der Funktion des syntaktischen Subjekts mit der Bezeichnung der situation in der Funktion des ersten syntaktischen 0bjekts verknüpft; $v g l$. Oper ${ }_{1}$ (mera) = prinimat (MEL'CUK 1974b, 92-95). Bei den LF Labor und Oper handelt es sich um sogenannte semantikentleerte, rein syntaktische Verknüpfungen. Aus diesem Grund können wir in (4) 'podvergat' ' durch 'prinimat'...protiv' substituieren, ohne die Gesamtbedeutung der lexikographischen Definition zu verändern:

(5) Tovarišc̆ Lyzñikov prinimaet meru vozdejstvija protiv tovariǒča Ivanova, soveršivšego prestuplenie, prostupok.'

Wir legen den Versuchspersonen die lexikographische Definition von nakazyvat, in der unter (5) geführten form zur Reduktionsparaphrasierung vor.

b) nakazyvat' (EKW)

(1) A nakazyvaet $B$ za C nekotorym $D=$ 'A pričinjaet $B$, soveršiv̌̌emu prostupok $C, z l 0 D$ s cel'ju ispravit' $B$ ili podobnych emu iic.' (MEL'ČK $1974 b, 134)$ 
Wir besetzen die in (1) ausgewiesenen Kasusrollen ' $A$ ' ('sub'), 'B' ('obj'), ' $C^{\prime}$ ('Motiv') und ' $D$ ' ('Mod') mit Wortformen der Lexeme tovarǐ̌c Ly̌̃nikov, tovarǐšc Ivanor, kontrabanda und lišenie zarplaty sowie dem anaphorischen Personalpronomen ego. In (1) tilgen wir den Semkomplex ' $i z_{i}$ podobnych emu $z_{i c}$ '. Hierzu sind wir berechtigt, da dieser Teil der Explikation - entsprechende Hinweise geben die Konstrukteure des MST leider nicht - wahrscheinlich auf den Abschreckungscharakter der Bestrafung abhebt und bei der von uns gewählten lexikalischen Auffuillung der Kasusrollen nicht aktualisiert wird. AuBerdem tilgen wir in (1) die Bedeutungskomponenten 'prostupok' und ' $z 20$ ', um Redundanzen zu vermeiden:

(1) 'Tovarišc Ly̌̌nikov pričinjaet tovarišcu Ivanovu, soveršiv̌̌emu kontrabandu, lišenie zarplaty s cel'ju ispravit' ego.'

In der unter (2) angeführten Form legen wir den Versuchspersonen die lexikographische Definition von nakazyvat' zur Reduktionsparaphrasierung vor.

\section{6. predlagat'}

a) predlagat" (OZEGOV)

(1) predlagat' = 'Predstavljat' na obsuždenie kak izvestnuju vozmožnost' ' (OZEGOV 1973, 532)

Wir expandieren (1) in (3), indem wir die Bedeutungskomponente 'izvestnuju' durch

(2) $\underline{i z v e s t n y j}=$ 'Takoj, o kotorom znajut, imejut svedenija' (OZEGOV 1973, 221)

ersetzen:

(3) 'Predstavijat' na obsuždenie kak takuju vozmožnost', o kotoroj znajut, imejut svedenija'

Wir besetzen die in (3) anzusetzenden Kasusrollen 'sub', 'Adr' und 'Content', deren syntaktische Realisierung obli- 
gatorisch ist, mit Wortformen der Lexeme Pëtr und Natǎ̌a sowie dem Syntagma pojti na sobranie und dem anaphorischen Personalpronomen ona. AuBerdem tilgen wir in (3) die redundanten Seme 'takuju' und 'znajut':

(4) 'Pëtr predstavljaet Nataše na obsuždenie kak vozmožnost', o kotoroj ona imeet suedenija, pojti na sobranie.'

In der unter (4) angeführten Form legen wir den Versuchspersonen die lexikographische Definition von predlagat' zur Reduktionsparaphrasierung vor.

b) predlagat' (EKW)

(1) X predlagaet $Y Z-u=' X$ soobščaet $2-u$, ̌́to, esli $z$ chočet proizvodit' dejstvija $Y$, vozmožnost' čego zavisit ot $X-a$, to $X$ kauziruet itu vozmožnost'.' (VOL'F 1973,4)

Die in (1) ausgewiesenen Kasusrollen ' $X$ ' ('sub'), ' $Y$ ' ('Content') und ' $Z$ ' ('Adr') besetzen wir mit Wortformen der Lexeme Pëtr und Nataša, dem Syntagma pojti na sobranie und den anaphorischen Personalpronomen ona, nego und on. In (1) tilgen wir die Bedeutungskomponenten 'proizvodit' dejstvija', um Redundanz zu vermeiden. AuBerdem substituieren wir in (1) die Bedeutungskomponente 'kauziruet' durch die Bedeutungskomponente 'pričinit' (vgl. S. 187):

(2) 'Pëtr soobsčăet Nataše, èto, esli ona chočet pojti na sobranie, vozmožnost' čego zavisit ot nego, to on pričinit estu vozmožnost'.

In der unter (2) angeführten Form legen wir den Versuchspersonen die lexikographische Definition von predlagat' zur Reduktionsparaphrasierung vor. 
7. prenebregat'

a) prenebregat' (OZEGOV)

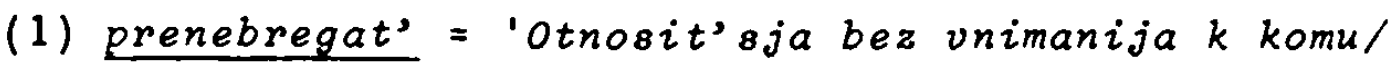
čemu-n., ne sčitat'sja s kem/ćem-n.' (OZEGOV 1973, 536)

Wir tilgen in (1) den redundanten Semkomplex 'ne sčitat'sja $s \mathrm{kem} / \check{c ̆ e m}^{-n}$. und expandieren (1) in (3), indem wir die Bedeutungskomponente 'unimanija' durch

(2) $\underline{\text { unimanie }}=$ 'Sosredotočenie myslej ili zrenija, slucha na kom/čëm-n.' (OZEGOV 1973, 81)

ersetzen:

(3) 'Otnosit'sja k komu/čemu-n. bez sosredotočenija myslej ili zrenija, slucha na kom/čëm-n.'

In (3) tilgen wir aufgrund der Referenzidentität von ' $k$ komu/čemu-n.' und 'na kom/čëm-n.' die Angabe 'na kom/ čëm-n.'. Wir besetzen die in (3) anzusetzenden Kasusrollen 'sub' und ' $O b j$ ', deren syntaktische Realisierung obligatorisch ist, mit Wortformen der Lexeme Larisa und zdorov'e:

(4) 'Larisa otnositsja bez sosredotočenija myslej ili zrenija, slucha $k$ zdorov'ju.'

In (4) tilgen wir den Semkomplex 'ili zrenija, slucha'. Wir sehen es als gesichertes Weltwissen an, daB diese Bedeutungskomponenten nicht aktualisiert werden, wenn die Kasusrolle 'obj' bei prenebregat' durch Wortformen von Lexemen wie beispielsweise zdorov'e, rabota, povedenie u.ä. eingenommen wird:

(5) 'Larisa otnositsja bez sosredotočenija myslej k zdoro$v^{\prime} j u .^{\prime}$

In der unter (5) angeführten form legen wir den Versuchspersonen die lexikographische Definition von prenebregat' zur Reduktionsparaphrasierung vor. 
b) prenebregat' (EKW)

(1) $y$ prenebregaet $X-o m=' Y$ dejstvujet, sčitaja, čto $X$ nesušcestvenen dija dejatel'nosti $\mathrm{Y}-a$.' (APRESJAN 1974b, 107)

Wir besetzen die in (1) ausgewiesenen Kasusrollen ' $y$ ' ('sub') und ' $X^{\prime}$ ('obj') mit Wortformen der Lexeme Larisa und zdorov'e sowie dem (anaphorischen) Possessivpronomen svoej:

(2) 'Larisa dejstvujet, sicitaja, čto zdorov'e nesuščestveno dija svoej dejatel'nosti.'

In der unter (2) angeführten form legen wir den Versuchspersonen die lexikographische Definition von prenebregat, zur Reduktionsparaphrasierung vor.

\section{8. provocirovat'}

a) provocirovat' (OZEGOV)

(1) provocirovat' 'Vyzyvat' na kakie-n. dejstvija putëm provokacii' (OZEGOV 1973, 558)

Wir expandieren ( 1 ) in (3), indem wir die Bedeutungskomponente 'provokacii' durch

(2) provokacija = 'Predatel'skoe povedenie, podstrekatel'stvo kogo-n. k takim dejstvijam, kotorye mogut poviě́, za soboj tja$\check{z} \ddot{e} l y e$ dlja nego posledstvija' (OZEGOV 1973, ebd.)

ersetzen:

(3) 'Vyzyvat' na kakie-n. dejstvija putëm predatel'skogo povedenija, podstrekatel'stva kogo-n. k takim

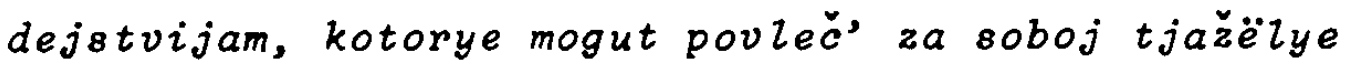
dija nego posledstuija'

Wir besetzen die in (3) anzusetzenden Kasusrollen 'sub' und ' $O b j$ ', deren syntaktische Realisierung obligatorisch 
ist, mit Wortformen der Lexeme gusar und sopernik:

(4) 'Gusar vyzyvaet sopernika na kakie-n. dejstvija putëm predatel'skogo povedenija, podstrekatel'stva sopernika $k$ takim dejstvijam, kotorye mogut povieč' za soboj tjažëlye dlja nego posledstvija.'

Von den beiden referenzidentischen Angaben in (4) 'na $k a k i e-n$. dejstvija' und ' $k$ takim dejstvijam' tilgen wir die letztere völlig und aus der ersteren die Bedeutungskomponente 'kakie-n.'. AuBerdem stellen wir den Semkomplex 'putëm predatel'skogo povedenija, podstrekatel'stva sopernika' an das Ende der Bedeutungsexplikation, um eventuelle Schwierigkeiten auszuräumen, die unsere Versuchspersonen bei der Reduktionsparaphrasierung der Definition aufgrund von deren geringer Kohärenz sonst haben könnten:

(5) 'Gusar vyzyvaet sopernika na dejstvija, kotorye mogut povieč' za soboj tjažëlye dija nego posledstvija,

putëm predatel'skogo povedenija, podstrekatel'stva sopernika.'

In der unter (5) angeführten Form legen wir den Versuchspersonen die lexikographische Definition von provocirovat' zur Reduktionsparaphrasierung vor.

\section{b) provocirovat' (EKW)}

(1) $X$ provociruet $Y-a$ na $Z$ = 'Imeja cel'ju kauzirovat' ušcerb $y-u, X$ pytaetsja kauzirovat' takoj prostupok 2 so storony $y-a$, kotoryj možet poslužit' opravdanijem dija realizacii étoj celi.' (APRESJAN 1974b, 107)

Wir besetzen die in (1) ausgewiesenen Kasusrollen ' $X$ ' ('sub'), ' $y$ ' ('obj') und ' $z$ ' ('Dest') mit Wortformen der Lexeme gusar, sopernik, draka und dem Possessivpronomen ego. AuBerdem substituieren wir in (1) die Bedeutungskomponente 'kauzirovat' durch 'pričinit' ' bzw. 
'pričinjat"' (vgl. S. 187) und tilgen den Semkomplex 'takoj prostupok', um Redundanz zu vermeiden:

(2) 'Imeja cel'ju pričinit' soperniku uščerb, gusar pytaetsja pričinjat' draku s ego storony, kotoraja možet poslužit' opravdanijem dlja realizacii étoj celi.'

In der unter (2) angeführten form legen wir den Versuchspersonen die lexikographische Definition von provocirovat' zur Reduktionsparaphrasierung vor.

9. razbivat'

a) razbivat' (OZEGOV)

(1) $\underline{\text { razbivat' }}=$ Sm. bit' (OZEGOV 1973, 589)

(2) $\underline{\text { bit' }}$ = 'Lomat', razdrobljat', raskalyvat' (OZEGOV $1973,48)$

Wir expandieren (2) in (4), indem wir die Bedeutungskomponente 'Zomat'" durch

(3) $\underline{\text { lomat' }}$ = 'sgibaja ili udarjaja s siloj, razdeljat' nadvoe, na časti, otdeljat' časti čego-n.' (OZEGOV 1973, 302)

ersetzen:

(4) 'Sgibaja ili udarjaja s siloj, razdeljat' nadvoe, na časti, otdeljat' časti čego-n., razdrabljat', raskalyvat" "

In (4) beseitigen wir die partiell redundanten Seme 'nadvoe' (Hyponym zu 'na časti'), 'otdeljat' časti čego-n.' (Hyponym zu 'razdeljat' na časti') und 'razdrabljat', raskalyvat' (Hyponym zu 'razdeljat' na časti'). Die in (4) anzusetzenden Kasusrollen 'sub' und ' $O b j$ ', deren syntaktische Realisierung obligatorisch ist, besetzen wir mit Wortformen der Lexeme Pëtr und zerkalo:

(5) 'Sgibaja ili udarjaja s siloj, Pëtr razdeljaet zerkalo na časti.' 
In der unter (5). angeführten form legen wir den Versuchspersonen die lexikographische Definition von razbivat, zur Reduktionsparaphrasierung vor.

b) razbivat' (EKW)

(1) A razbivaet $X=$ 'Udarjaja $x$-om ili po $x-u$, A delit tvërdyj $x . '$ (APRESJAN 1974b, 108)

In (1) tilgen wir, um Redundanz auszuschalten, die Bedeutungskomponente 'tvërdyj'. Die in (1) ausgewiesenen Kasusrollen ' $A$ ' ('Sub') und ' $X$ ' $(' O b j$ ') besetzen wir mit Wortformen der Lexeme Pëtr und zerkalo sowie dem anaphorischen Personalpronomen ego:

(2) 'Udarjaja zerkalom ili po zerkalu, Pëtr delit ego.'

In der unter (2) angeführten Form legen wir den Versuchspersonen die lexikographische Definition von razbivat' zur Reduktionsparaphrasierung vor.

10. $\underline{u b e \check{z} d a t^{\prime}}$

a) ubě̌dat’ (OŽEGOV)

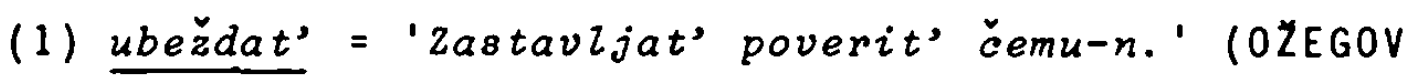
$1973,753)$

Wir expandieren (1) in (3), indem wir die Bedeutungskomponente 'zastavljat"' durch

(2) $\underline{\text { zastavljat' }}$ = 'Stavit' $v$ neobchodimost' delat' čto-n., prinudit" (OZEGOV 1973, 204)

ersetzen:

(3) 'Stavit' $v$ neobchodimost' delat' čto-n., prinudit' poverit' čemu-n.'

In (3) tilgen wir die redundante Bedeutungskomponente 'prinudit' . AuBerdem ersetzen wir den Semkomplex 'delat' čto-n.' durch 'poverit' čemu-n.', da 'dezat' in (2) bzw. (3) in der Funktion eines Proverbs verwendet wird und bei der Einbettung von (2) in (1) 'poverit' cemu-n.' Hyponym zu 
'dezat' čto-n.' ist:

(4) 'stavit' $v$ neobchodimost' poverit' čemu-n.'

Die in (4) anzusetzenden bzw. angesetzten Kasusrollen 'sub', 'obj' und 'Content', deren syntaktische Real isierung obligatorisch ist, besetzen wir mit Wortformen der Lexeme Pëtr und Natăsa sowie mit dem Syntagma čto on ne vinovat:

(5) 'Pëtr stavit Natašu v neobchodimost' poverit', čto on ne vinovat.'

In der unter (5) angefürten form legen wir den Versuchspersonen die lexikographische Definition von ubě̌dat' zur Reduktionsparaphrasierung vor.

(b) ubezdat' (EKW)

(1) A ubě̌daet $x-a v y-e=$ 'Privodja argumenty, $A$ pytaetsja kauzirovat' $X-a$ sčitat' Y.' (APRESJAN 1974b, 84)

Wir substituieren in (1) die Bedeutungskomponente 'kauzirovat" durch 'pričinjat"' (vgl. S. 187) und besetzen die ausgewiesenen Kasusrollen ' $A$ ' ('sub'), ' $X$ ' ('obj') und ' $y$ ' ('Content') mit Wortformen der Lexeme Pëtr, Nataša und dem Syntagma čto on ne vinovat:

(2) 'Privodja argumenty, Pëtr pytaetsja pričinjat' Nată̌u sčitat', čto on ne vinovat.'

In der unter (2) angefiurten Form legen wir den Versuchspersonen die lexikographische Definition von ubězdat' zur Reduktionsparaphrasierung vor.

11. udarjat'

a) udarjat' (OZZEGOV)

(1) $\underline{\text { udarjat' }}=$ 'Nanosit' udar komu-n., proizvodit' udar obo čto-n.' (OZEGOV 1973, 758)

Wir expandieren (1) in (3), indem wir die Bedeutungskom- 
ponente 'udar' durch

(2) $\underline{\text { udar }}=$ 'Rezkij, sil'nyj tolčok, prikosnovenie k komul čemu-n. s siloj' (OZZEGOV 1973, ebd.)

ersetzen. AuBerdem beseitigen wir in (1) den redundanten Semkomplex 'nanosit' udar komu-n.', da er mit 'proizvodit' udar obo čto-n.' synonym ist: ${ }^{49}$

(3) 'Proizvodit' reakij, sil'nyj tolčok, prikosnovenie $k$ komu/čemu-n. s siloj'

In (3) tilgen wir von den beiden redundanten Bedeutungskomponenten 'rezkij' und 'siz'nyj' die letztere. Die syntaktische Verknüpfung der Semkomplexe 'proizvodit' rezkij tolcook' und 'prikosnovenie k komu/čemu-n. s siloj', die in der OZEGOVschen lexikographischen Definition durch ein Komma ausgedrückt wird, interpretieren wir als eine attributive Verknuipfung. Aufgrund der durch 'udarjat' typischerweise bezeichneten Situation sehen wir es für die Bedeutung des Verbs als paradigmatisch an, daB die Beruhrung des Gegenstands, in dessen Richtung der Schlag erfolgt, unter gleichzeitiger Ausführung eines kräftigen StoBes mit demselben Ziel vor sich geht. Um diese Bedeutungsrelation zwischen beiden oben genannten Semkomplexen auszudrücken, schreiben wir (3) in eine präsentische Gerundialkonstruktion um (vgl. S. $161 \mathrm{ff.}$ ):

(4) 'Proizvodja rezkij tolčok, prikasat'sja k komulčemu-n. 8 si $20 j$ '

Die in (4) anzusetzenden Kasusrollen 'sub' und 'Obj', deren syntaktische Realisierung obligatorisch ist, besetzen wir mit Wortformen der Lexeme učitel' und stol:

\section{(5) 'Proizvodja rezkij tolčok, učitel' prikasaetsja s $8 i \operatorname{lojk}$ stolu.}

In der unter (5) angeführten form legen wir den Versuchspersonen die lexikographische Definition von udarjat' zur Reduktionsparaphrasierung vor. 
b) udarjat' (EKW)

(1) A $\underline{\text { udarjaet }}$ po $B-u$ C-om = 'A rezko $i$ kratkovremenno privodit kompaktnyj predmet $C v$ kontakt $s$ predmetom $B . '$ (APRESJAN 1974b, 108)

Wir besetzen die in (1) ausgewiesenen Kasusrollen ' $A$ ' ('sub'), ' $B$ ' ('Obj') und ' $C$ ' ('Instr') mit Wortformen der Lexeme ucitel', stol und ukazka. AuBerdem tilgen wir in (1) die Bedeutungskomponenten 'kompaktnyj predmet' und 'predmetom', um Redundanzen zu vermeiden:

(2) 'Učitel' rezko i kratkovremenno privodit ukazku $\underline{v}$ kontakt so stolom.'

In der unter (2) angeführten form legen wir den Versuchspersonen die lexikographische Definition von udarjat' zur Reduktionsparaphrasierung vor.

12. učityvat"

a) učityvat’ (OZEGOV)

(1) $\underline{\text { učityvat' }}$ = 'Prinimat' vo vnimanie' (OZZEGOV 1973, 777) Wir expandieren ( 1 ) in ( 3 ), indem wir die Bedeutungskomponente 'unimanie' durch

(2) $\underline{\text { unimanie }}=$ 'Sosredotočenie myslej ili zrenija, slucha na kom/čëm-n.' (OZEGOV 1973, 81)

ersetzen:

(3) "Prinimat' vo sosredotočenie myslej ili zrenija, slucha na kom/čëm-n.'

Die Substitution der Bedeutungskomponente 'unimanie' durch (2) ergibt die nichtwohlgeformte lexikographische Definition (3). Wir verändern (3), indem wir die Bedeutungskomponente 'prinimat' vo (sosredotočenie)' durch das Sem 'napraviajat' (sosredotočenie) na' ersetzen. Unsere Modifikation läßt die Gesamtbedeutung der lexikographischen Definition (3) unverändert, sie berichtigt nur die in ihr 
enthaltenen Verstöße gegen Normen der Kombinierbarkeit russischer Lexeme. Ausgedrückt in den Termini der MEL'CUKschen lexikalischen Funktionen zielen unsere Veränderungen lediglich darauf ab, anstelle von 'prinimat' den für sosredotočenie richtigen Wert der lexikalischen Funktion oper, ${ }_{1}$ (sosredotočenie) = napravijat' in (3) einzusetzen:

(4) 'Napravljat' sosredotočenie myslej ili zrenija, slucha na kogo/čto-n.'

Die in (4) anzusetzenden Kasusrollen 'sub' und 'Obj', deren syntaktische Realisierung obligatorisch ist, besetzen wir mit einer Wortform des Lexems chimik und dem Syntagma vyvody kolleg. Wir nehmen auBerdem als gesichertes Weltwissen an, daß bei dieser lexikalischen Auffüllung der Kasusrollen 'sub' und ' $O b j$ ' in der durch učityvat' typischerweise bezeichneten Situation die Bedeutungskomponente 'slucha' nicht aktualisiert wird und daher getilgt werden kann. M.a.W., wir gehen davon aus, daB vyvod in dem hier vorgegebenen kontext nicht münlich vorgetragene SchluBfolgerungen bezeichnen kann:

(5) 'Chimik napravljaet sosredotočenie myslej ili zrenija na vyvody kolleg.

In der unter (5) angeführten form legen wir den Versuchspersonen die lexikographische Definition von ucityvat, zur Reduktionsparaphrasierung vor.

b) ǔ́ityvat' (EKW)

(1) y učityvaet $x=' y$ dejstvuet, sčitaja, čto $x$ suščestvenen dlja dejatel'noeti $Y-a$.' (APRESJAN 1974b, 109)

Die in (1) ausgewiesenen Kasusrollen ' $y$ ' ('sub') und ' $x$ ' ('obj') besetzen wir mit einer Wortform des Lexems chimik, dem Syntagma vyvody kolzeg und dem Possessivpronomen svoej:

(2) 'Chimik dejstvuet, sčitaja, čto vyvody kolleg suščestveny dija svoej dejatel'nosti. 
In der unter (2) angeführten form legen wir den Versuchspersonen die lexikographische Definition vun ucityvat, zur Reduktionsparaphrasierung vor.

13. učit'sja

a)

(1) $\underline{\text { učit'sja }}$ = 'Usvaivat' kakie-n. znanija, navyki' (OŽEGOV 1973, 777)

Wir expandieren (1) in (3), indem wir die Bedeutungskomponente 'usvaivat'" durch

(2) $\underline{\text { usvaivat' }}$ = 'Ponjav, zapominat' kak sleduet' (OZEGOV 1973, 770)

ersetzen:

(3) 'Ponjav, zapominat' kak sleduet kakie-n. znanija, navyki'

Im Fall von učit'sja ermöglicht das Wöterbuch von OZEGOV noch eine weitere Entfaltung der Bedeutungsexplikation. Wir können das Sem 'zapominat' in (3) durch

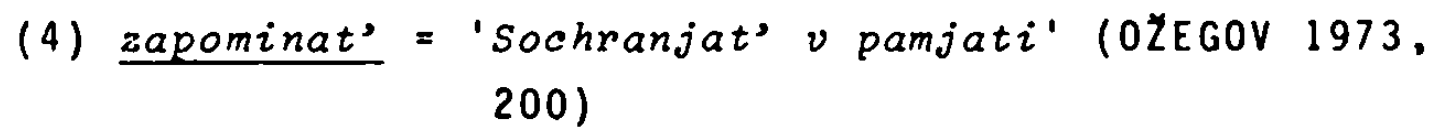
ersetzen:

(5) 'Ponjav, sochranjat' $v$ pamjati kak sleduet kakie-n. znanija, navyki'

Die in (5) anzusetzenden Kasusrollen ' $s u b^{\prime}$ und ' $O b j$ ', deren syntaktische Realisierung obligatorisch ist, besetzen wir mit Wortformen der Lexeme Nataša und matematika. In (5) tilgen wir die Bedeutungskomponenten 'kakie-n. znanija, navyki', um Redundanzen auszuschalten:

(6) 'Ponjav, Nataša sochranjaet matematiku v pamjati kak sleduet.'

In der unter (6) angefürten form legen wir den Versuchspersonen die lexikographische Definition von učit'sja zur 
Reduktionsparaphrasierung vor.

b) $u \dot{c} i t^{\prime} s j a$ (EKW)

(1) $x$ učitsja $y-u=' x$, soznatel'no zatračivaja usilija, stremitsja priobresti znanija $i i_{i}$ umenija Y.' (MEL'CUK 1974b, 137)

Wir besetzen die in (1) ausgewiesenen Kasusrollen ' $X$ ' ('sub') und ' $Y$ ' ('Content') mit Wortformen der Lexeme Nataša und matematika. GemäB der im EKW für die Gruppe der Verben des Lehrens und Lernens angegebenen Tilgungsregel streichen wir in (1) den Semkomplex 'ili umenija', da es sich bei dem Lexem matematika um eine Bezeichnung für ein Wissensgebiet ('znanija') handelt (APRESJAN 1974b, 84-85):

(2) 'Natắa, soznatel'no zatračivaja usilija, stremitsja priobresti matematiku.'

In der unter (2) angefihrten Form legen wir den Versuchspersonen die lexikographische Definition von učit'sja zur Reduktionsparaphrasierung vor.

\section{4. ostorožnyj}

a) ostorožnyj (OŽEGOV)

(1) ostorǒ̌nyj = 'Predusmatrivat' vozmožnuju opasnost', ne oprometčivyj' (OZEGOV 1973, 424)

Wir expandieren (1) zunächst in (6), indem wir die Bedeutungskomponente 'opasnost' ' in mehreren Schritten entfalten:

(2) opasnost' $^{\prime}=S m$. opasnyj (OZEGOV 1973, 413)

(3) opasnyj = 'Sposobnyj vyzvat', pričinit' kakoj-n. vred, nesčast'e' (OŽEGOV 1973, ebd.)

Wir expandieren (3) in (5), indem wir die Bedeutungskomponente 'nesčast'e durch 


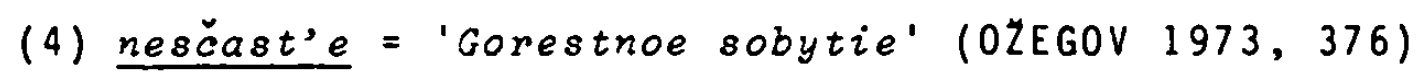

ersetzen und in (3) das redundante Sem 'vizuat" tilgen:

(5) 'Sposobnyj pričinit' kakoj-r. ired, gorestnoe sobytie'

Wir ersetzen in (1) die Bedeutungskomponente 'opasnost" durch (5). Da wir bei der Expandierung von 'opasnost" im Wörterbuch von OZEGOV dem Verweis opasnost' $=\mathrm{sm}$. opasnyj folgen mußten, fügen wir in (1) das Sem 'situacija' ein. Mit dieser Maßnahme verändern wir die Gesamtbedeutung der lexikographischen Definition von opasnyj (3), jedoch nur im Sinne einer Reduzierung des Bedeutungsumfangs und somit des Referenzpotentials von opasnyj, weil wir die Klasse der möglichen Argumente des Prädikats opasnyj auf die der Bezeichnungen für Situationen einengen. Es ist erforderlich, in (1) das Sem 'situacija' einzufügen, um sicherzustellen, daß die lexikographische Definition nach entsprechender Expandierung durch (5) wohlgeformt bleibt. M.a.W., wir beheben durch diese Modifikation lediglich die Folgen, die der Wortklassenwechsel in OZEGOVs Verweis, zur Explikation der Bedeutung von opasnost' die lexikographische Definition von opasnyj aufzusuchen, für die Konstruktion unseres Materials nach sich zieht:

(6) 'Predusmatrivat' vozmožnuju situaciju, sposobnuju pričinit' kakoj-n. vred, gorestnoe sobytie'

Im Falle der lexikographischen Definition von ostorožnyj bietet das Wörterbuch von OZZEGOV die Möglichkeit, noch eine weitere Bedeutungskomponente zu expandieren. Wir entfalten (6) in (8), indem wir das Sem 'predusmatrivat"' durch

(7) predusmatrivat' $=$ 'Predvidja, prigotovijat'sja $\mathrm{k}$ čemu-n.' (OZEGOV 1973, 534)

ersetzen:

(8) 'Predvidja, prigotovljat'sja k vozmožnoj situacii, sposobnoj pričinit, kakoj-n. vred, gorestnoe sobytie' 
Die in (8) anzusetzende Kasusrolle 'sub', deren syntaktische Realisierung obligatorisch ist, besetzen wir mit einer Wortform des Lexems Tanja:

(9) 'Predvidja, Tanja prigotovijaetsja k vozmožnoj situacii, sposobnoj pričinit' kakoj-n. vred, gorestnoe sobytie.'

In der unter (9) angefühten Form legen wir den Versuchspersonen die lexikographische Definition von ostorožnyj zur Reduktionsparaphrasierung vor.

b) ostorožnyj (EKW)

(1) $X$ ostorožen $=$ ' $X-u$ svojstvenno želanie izbegat' situacij, kotorye mogut byt' opasnymi ili neprijatnymi dija $x-a$, i poétomu $X$ staraetsja dejstvovat' s uöëtom vsech tech faktorov, kotorye, po ego mneniju, mogut kauzirovat' takuju situaciju.' (APRESJAN 1974b, 107 )

Wir besetzen die in (1) ausgewiesene Kasusrolle ' $X$ ' ('sub') mit einer Wortform des Lexems Tanja, den anaphorischen Personalpronomen neë, ona und $e \ddot{e}$ und substituieren die Bedeutungskomponente 'kauzirovat' durch das Sem 'pričinit'" (vgl. S. 187):

(2) 'Tane svojstvenno želanie izbegat' situacij, kotorye mogut byt' opasnymi $i z_{i}$ neprijatnymi dlja ne $\ddot{e}, i$ poétomu ona staraetsja dejstvovat' s učëtom vsech tech faktorov, kotorye, po ë̈mneniju, mogut pričinit' takuju situaciju.'

In der unter (2) angeführten Form legen wir den Versuchspersonen die lexikographische Definition von ostorožnyj zur Reduktionsparaphrasierung vor.

\section{Prő̌nyj}

a) pročnyj (OZEGOV)

(1) pročnyj $=' K r e p k i j$, trudno razrušajuščijsja (OZ̈EGOV 1973, 578) 
Wir expandieren (1) in (3), indem wir die Bedeutungskomponente 'razruisajuščijsja' durch

(2) $\underline{\text { razrušat' }}=$ 'Presvraščat' v razvaliny' (OZEGOV 1973, 602)

ersetzen:

(3) 'Krepkij, trudno prevraščajuščijsja v razvaliny'

In (3) tilgen wir die redundante Bedeutungskomponente

'krepkij'. AuBerdem besetzen wir die in (1) anzusetzende Kasusrolle 'Sub', deren syntaktische Realisierung obligatorisch ist, mit einer Wortform des Lexems kolonna:

(4) 'Kolonna trudno prevraščajuščajasja v razvaliny.

In der unter (4) angeführten form legen wir den Versuchspersonen die lexikographische Definition von pročnyj zur Reduktionsparaphrasierung vor.

b) pročnyj (EKW)

(1) $x$ prōenen $=$ 'x takoj, kotoryj trudno razrušaetsja mechaničeskoj siloj.' (APRESJAN 1974, 109)

Wir besetzen die in (1) angesetzte Kasusrolle 'Sub', deren syntaktische Realisierung obligatorisch ist, mit einer Wortform des Lexems kozonna:

(2) 'Kolonna takaja, kotoraja trudno razrušaetsja mechaničeskoj siloj.'

In der unter (2) angeführten form legen wir den Versuchspersonen die lexikographische Definition von pročnyj zur Reduktionsparaphrasierung vor.

16. spravedlivyj

a) spravedlivyj (OZEGOV)

(1) spravedlivyj $=$ 'Dejstvujuščij bespristrastno $v$ sootvetstuii s istinoj' (OZEGOV 1973, 697) 
Wir expandieren (1) in (3), indem wir die Bedeutungskomponente 'istinoj' durch

(2) $\underline{\text { istina }}=$ 'To, čto sušcestvuet v dejstvitel'nosti, otražaet dejstvitel'nost', pravda' (OŽEGOV 1973,236 )

ersetzen:

(3) 'Dejstvujušcijj bespristrastno, v sootvetstvii s tem, čto suřçsstvuet $v$ dejstvitel'nosti, otražaet dejstvitel'nost', pravdu'

In (3) tilgen wir die redundanten Bedeutungskomponenten ',otražaet dejstvitel'nost', pravdu. 'Die in (3) anzusetzende Kasusrolle 'Sub', deren syntaktische Realisierung obligatorisch ist, besetzen wir mit einer Wortform des Lexems Tanja:

(4) 'Tanja - dejstvujuščaja bespristrastno, v sootvetstviis tem, čto sušcestvuet $v$ dejstvitel'nosti. '

In der unter (4) angeführten form legen wir den Versuchspersonen die lexikographische Definition von spravedizivj zur Reduktionsparaphrasierung vor.

b) spravedlivyj (EKW)

(1) $X$ spravedlivyj $=$ ' $X$ sposobnyj pravil'no ocenit', kak sleduet dejstvovat', čtoby nikakim ljudjam ne bylo kauzirovano nezaslužennoe zlo.' (APRESJAN 1974b, 107)

Wir substituieren in (1) die Bedeutungskomponente

'kauzirovano' durch 'pričineno' (vgl. S. 187). AuBerdem besetzen wir in (1) die Kasusrolle ' $X$ ' ('sub') mit einer Wortform des Lexems Tanja:

(2) 'Tanja sposobnaja pravil'no ocenit', kak sleduet dejstvovat', čtoby nikakim ljudjam ne bylo pričineno nezaslužennoe zlo. 
In der unter (2) angeführten Form legen wir den Versuchspersonen die lexikographische Definition von spravediivyj zur Reduktionsparaphrasierung vor.

17. $\underline{\text { jasnyj }}$

a) jasnyj (OZ̈EGOV)

(1) $\underline{j a s n y j}=$ 'Svetlyj, ničem ne zatemnënnyj' (OŽEGOV 1973, 842)

Wir expandieren (1) in (3), indem wir die Bedeutungskomponente 'zatemnënny $j$ ' durch

(2) $\underline{\text { zatemnjat' }}=$ 'Delat' tëmnym ili zakryvat' ̌̌em-n. tëmnym, ne propuskajuščim sveta' (OZEGOV 1973, 206)

ersetzen:

(3) 'Svetlyj, ničem ne sdelannyj tëmnym ili nǐcem tëmnym ne zakrytyj, ne propuskajuščim sveta'

In (3) tilgen wir die redundante Bedeutungskomponente 'suetzyj'. AuBerdem besetzen wir die in (3) anzusetzende Kasusrolle ('sub'), deren syntaktische Realisierung obligatorisch ist, mit einer Wortform des Lexems nebo:

(4) 'Nebo ničem ne sdelano tëmnym ili ničem tëmnym ne zakryto, ne propuskajušcim sveta.

In der unter (4) angefühten Form legen wir den Versuchspersonen die lexikographische Definition von jasnyj zur Reduktionsparaphrasierung vor.

b) jasnyj (EKW)

(1) $j a s n y j x=' x$ - charakterizujuščijsja otsutstviem $v$ atmosfere častic (naprimer/= oblaka, pyl', tuman, dym ...), prepjatstvujuščich vosprijatiju sveta.' (KOSSEK 1973, 38)

Wir besetzen die in (1) angesetzte Kasusrolle ' $X$ ' ('sub') mit einer Wortform des Lexems nebo: 
(2) 'Nebo - charakterizujuščeesja otsutstviem v atmosfere castic loblaka, pyl', tuman, dym ....), prepjatstvujušcich vosprijatiju sveta. '

In der unter (2) angeführten form legen wir den Versuchspersonen die lexikographische Definition von jasnyj zur Reduktionsparaphrasierung vor. 


\section{7um Verlauf des EXPERiments}

\subsection{Prinzipien der Durchfïhrung des Tests}

Wir haben unser Experiment mit insgesamt 12 Versuchspersonen durchgeführt, die wir in zwei Gruppen zu je 6 Informanten aufgeteilt haben. Der einen Gruppe legten wir die lexikographischen Definitionen des EKW, der anderen Gruppe die lexikographischen Definitionen des Wörterbuchs von OZEGOV zur Reduktionsparaphrasierung vo.

Die Ergebnisse der Befragung von 12 Informanten können selbstverständich nicht als hinreichende Stichprobe angesehen werden, die eine Grundlage für statistische Auswertungen böte. Neben den bereits genannten Gesichtspunkten der Signifikanzund Validitätsproblematik (vgl. u.a. S. 141, Anm. 34, S. $146 \mathrm{ff.}, \mathrm{S} .152 \mathrm{ff.}$ ) liegt in der geringen Datenbasis unserer Erhebung ein weiterer Grund, unseren Versuch lediglich als eine Vorausanalyse zu betrachten, die mögliche Verfahrensweisen für die zukünftige Forschung in der linguistischen Semantik aufzeigen soll. 50

Bei der Auswahl der Versuchspersonen haben wir uns, wie bereits erwähnt, an den Homogenitätskriterien Alter und Schulbildung orientiert. Wir haben festgelegt, nur solche Sprecher des Russischen zu befragen, die mindestens 18 Jahre alt sind und das sowjetische Abitur (attestat zrezosti), d.h. den AbschluB der zehnklassigen srednjaja : : kola nachweisen konnten. Die spezifischen geographischen Bedingungen, unter denen wir unseren Versuch durchgeführt haben, machten es nötig, noch eine weitere Forderung zu erheben, der die Informanten genügen muBten. Wir haben nur solche Sprecher des Russischen zu unserem Experiment zugelassen, die glaubhaft machen konnten, das Russische als Muttersprache erworben zu haben und es heute auch noch als solche zu verwenden. Alle 12 Informanten, mit denen wir unseren Versuch durchgeführt haben, genügten den drei von uns aufgestellten Homogenitätskriterien. Es handelte sich im einzelnen um 8 Frauen und 4 Männer im Alter 
Zwischen 19 und 37 Jahren, die alle das sowjetische Abitur abgelegt hatten. Obwohl sie teils ständig im deutschen Sprachraum lebten, gaben alle Versuchspersonen an, sich ausschlieblich des Russischen zu bedienen, um ihre familiären und gesellschaftlichen Kontakte aufrechtzuerhalten, und das Deutsche nur in Situationen wie z.B. im beruflichen Umfeld zu verwenden, in denen es unbedingt erforderlich sei. 51

Bei der Durchfihrung unseres Versuchs haben wir uns bemint, die sprachliche Vorstellungskraft und Aufmerksamkeit der Versuchspersonen so gut wie möglich auf den Gegenstand unseres Experiments, die SemS des EKW und die Definitionssprache des Wörterbuchs von OZEGOV, zu lenken. Um dies zu erreichen, haben wir mehrere Maßnahmen ergriffen, die auf die Konstruktion. des Testmaterials, seine Anordnung und Präsentation während der Durchfihrung des Versuchs und die Testanweisung abzielten.

Wir haben, wie bereits besprochen (vgl. S. 142), das Versuchsmaterial so konstruiert, daB redundante Bedeutungskomponenten in den zu testenden lexikographischen Definitionen eliminiert worden sind. Neben ihrer Absicherung durch lexikologische Prinzipien, die sowohl das EKW als auch das Wörterbuch von OZEGOV für ihre lexikographische Praxis geltend machen ( $v g l$. S. 152 f.), halten wir diese Maßnahme für erforderlich, da wir vermuten, daB redundante Bedeutungskomponenten die Aufmerksamkeit der Informanten zu stark ablenken, ihre sprachliche Vorstellungskraft negativ beeinflussen und somit ihre Fähigkeit, Reduktionsparaphrasierungen zu vollziehen, beeinträchtigen (vgl. S. $176 \mathrm{f.}$ ).

Eine zusätzliche, auf die Konstruktion des Testmaterials abzielende Maßnahme bestand darin, daß wir die Kasusrollen in den Bedeutungsexplikationen des EKW und des Wörterbuchs von OZEGOV mit konkreten Wortformen bestimmter Lexeme aufgefiilt haben (vgl. S.

Bei der Durchfiuhrung unseres Tests ordneten wir das Versuchsmaterial so, daB den Informanten die lexikographischen Definitionen zur Reduktionsparaphrasierung in einer Reihenfolge 
vorgelegt wurden, die möglichst große zeitliche Abstände $z w i-$ schen Bedeutungsexplikationen ergab, die zum Teil identische Bedeutungskomponenten enthielten. Auf diese Weise wollten wir verhindern, daB die Versuchspersonen durch dicht aufeinanderfolgende, sehr ähnliche lexikographische Definitionen zu Interferenzleistungen verleitet oder ganz allgemein verwirrt wiurden.

Wir legten unseren Informanten die zu paraphrasierenden Bedeutungsexplikationen auf Karteikarten abgedruckt vor. Diejenigen Bedeutungskomponenten, die auf ein Verb bzw. ein Adjektiv des Russischen reduziert werden sollten, haben wir durch Unterstreichen visuell besonders hervorgehoben (vgl. S. 144).

Zu Beginn unseres Experiments gaben wir den Versuchspersonen eine zweiteilige Testanweisung. Ihr erster Teil beinhaltete die Instruktion in abstrakter Form, der zweite illustrierte anhand je eines Beispiels für die Bedeutungsexplikation eines Verbs und eines Adjektivs, welche Operation mit dem Testmaterial durchzuführen war. Im ersten Teil der Testanweisung gaben wir der Informantengruppe, die lexikographische Definitionen des EKW Zu paraphrasieren hatte, noch die zusätzliche Erläuterung, daB eventuell mit grammatischen Fehlern in den Bedeutungsexplikationen zu rechnen sei (vgl. S. 140).

Die Testanweisung hatte den folgenden Wortlaut:

1. Predvaritel'nye zamečanija

Mne chotelos' by provesti s Vami dva éksperimenta. Ja dam Vam predloženija na russkom jazyke. Prošu Vas preobrazovat' et $i$ predloženija po sledujuščim instrukcijam. (Nekotorye iz predloženij mogut soderžat' grammatičeskie ošibki, kotorye, odnako, dlja našich celej, nevažny. ${ }^{52}$

\subsection{Instrukcii $k$ pervomu éksperimentu}

Sokratite sledujuščie predloženija, zamenjaja sovokupnost' podčërknutych slov ili vyraženij odnim glagolom russkogo jazyka i sochranjaja vse ostal'nye. Vremja dija razmyšlenija - dve minuty. Napišite predloženie s iskomym glagolom na kartočke. 


\subsection{Obrazec}

Ischodnoe predloženie:

Student peremešcaetsja iz instituta $v$ stolovuju, perestupaja nogami $i$ ni $v$ kakoj moment ne utračivaja polnost'ju kontakta s poverchnost' $j u$, po kotoroj student peremě̌čaetsja.

Resul'tat sokrašcenija:

Student idët iz instituta $v$ stolovuju.

Iskomyj glagol - glagol "idti"

\subsection{Instrukcii $k$ vtoromu èksperimentu}

Sokratite sledujušcie predloźenija, zamenjaja sovokupnost" podčërknutych slov ili vyraženij odnim prizagatel'nym russkogo jazyka i sochranjaja vse ostal'nye. Vremja dija razmy̌̆lenija - dve minuty. Napišite predloženie 8 iskomym prilagatel'nym na kartočke.

\section{2 obrazec}

Ischodnoe predloženie:

Al'pinist obladaet sposobnost'ju bystro $i$ bez truda koordinirovat' dvizenija svoego tela pri vypolnenii fizičeskich dejstvij.

Resul'tat sokraščnija:

Al'pinist lovkij.

Iskomoe prilagatel'noe - prilagatel'noe "lovkij".

Wir druckten die Testanweisung entsprechend der oben genannten Gliederung auf éinzelnen Karteikarten ab. Zu Beginn unseres Versuchs legten wir den Informanten die Instruktion in der Reihenfolge 1., 2.1,2.2, 3.1 und 3.2 vor. Die Versuchspersonen hatten auBerdem während der gesamten Dauer des Experiments die Möglichkeit, die Testanweisung nochmals einzusehen.

Bei der Aufgliederung der Testanweisung in zwei Teile haben wir ihren ersten Teil, der die Instruktion in abstrakter form enthält, bewuBt so abgefaBt, daB er im Hinblick auf die sprachlichen Operationen, die die Versuchspersonen durchführen sollen, unterbestimmt ist. In 2.1 und 3.1 fehlt die Teilinstruk- 
tion, die syntaktische Funktion von Wortformen derjenigen Lexeme, die bei der Reduktionsparaphrasierung invariant bleiben sollen, nicht zu verändern. Außerdem haben wir darauf verzichtet, die Versuchspersonen anzuweisen, im Falle von wiederholtem Vorkommen gleicher oder verschiedener Wortformen desselben Lexems, die von der Umformung nicht affiziert werden sollten, diese in der Reduktionsparaphrase nur einmal zu nennen ( $v g l$. S. 229 razbivat').

Indem wir diese Informationen den Versuchspersonen in expliziter Form vorenthalten haben, wollten wir vermeiden, die Testanweisung für die Informanten in unnötiger Weise zu komplizieren. Aus diesem Grund haben wir die Instruktion mit dem illustrativen Teil versehen, der den Versuchspersonen die oben genannten Informationen in impliziter form zur verfügung stellt.

Es sei in diesem Zusammenhang noch angemerkt, daB es für eine Reduktionsparaphrasierung als solche unerheblich ist, ob sich die syntaktischen Funktionen von Wortformen derjenigen Lexeme, die durch die Umformung nicht affiziert werden sollen, ändern oder erhalten bleiben. Im folgenden Beispiel ist (2) mit gleichem Recht wie ( $\left.2^{\prime}\right)$ als eine Reduktionsparaphrase von (1) anzusehen:

(1) 'Policija v sootvetetvii s predvaritel'nym rasporjaženiem lišila Petra svobody za vorovstuo.

(2) Pëtr popal $v$ ruki policii za vorovstvo.

(2') Policija arestovala Petra za vorovetvo.

Für unsere Zwecke ist es jedoch äuBerst wichtig, daß die Wortformen derjenigen Lexeme, die von der Paraphrasierung nicht betroffen worden sind, hinsichtlich ihrer syntaktischen Funktionen invariant bleiben. Wir wollen unsere Aussagen uber die Verstehbarkeit der Definitionssprache des Wörterbuchs von OZEGOV und der SemS des EKW in Abhängigkeit davon treffen, ob die Bedeutungsrelation, die zwischen bestimmten Sätzen mit dem gesuchten Stichwort und den jeweils von den Versuchspersonen genannten Reduktionsparaphrasen besteht, als Synonymie 
oder Nichtsynonymie beurteilt werden kann (vgl. S. 144). Da es sehr.wahrscheinlich ist, daB im Laufe des Versuchs auch solche Reduktionsparaphrasen genannt werden, die ein anderes verb bzw. Adjektiv als das gesuchte Stichwort enthalten, das jedoch mit diesem in dem gegebenen Kontext bedeutungsgleich ist, muissen wir bei der Anlage unseres Experiments unbedingt darauf achten, $d a B$ die im Test zu erwartenden Reduktionsparaphrasen auch bezijglich dieser Bedeutungsrelation klassifiziert werden können. Zu diesem Zweck wollen wir uns des Urteils eines russischen Muttersprachlers als Informanten bedienen (vgl. S. $232 \mathrm{ff}$ ). Wir halten es daher für erforderlich, keine Veränderung der syntaktischen Funktionen von Wortformen derjenigen Lexeme zuzulassen, die nicht unter die Umformung fallen. Durch diese Auflage an die Versuchspersonen schlieBen wir Reduktionsparaphrasen wie beispielsweise (2) aus, die als Konversion von (1) syntaktische Umarrangierungen der Ausdruicke enthält, die für die durch arestovyvat' bezeichnete Situation paradigmatische Aktanten realisieren. Indem wir die Reduktionsparaphrasierungen der Versuchspersonen auf reine Substitutionen beschränken, schaffen wir eine geeignete Grundlage, um in all den Fällen, in denen in einer Reduktionsparaphrase ein anderes als das gesuchte Stichwort genannt wurde, die Testergebnisse durch unseren Informanten hinsichtlich der Bedeutungsrelation, die zwischen dieser Reduktionsparaphrase und dem entsprechenden Satz mit dem gesuchten Stichwort besteht, beurteilen zu lassen. Wir haben durch unsere MaBnahme den potentiellen Störfaktor ausgeschaltet, daB unterschiedliche syntaktische Funktionen von Wortformen bestimmter Lexeme in den Satzparen, die wir dem Informanten zur Bewertung vorlegen, dessen Urteil uber das Vorhandensein von Synonymie bzw. Nichtsynonymie zwischen den Sätzen negativ beeinflussen könnten.

Um die Durchfïhrung unserer Experimente zeitlich zu begrenzen, setzten wir die Bedenkzeit der Versuchspersonen für jede einzelne zu paraphrasierende lexikographische Definition auf maximal 2 Minuten fest. Diese Zeit wurde jedoch von keinem der Informanten auch nur annähernd in Anspruch genommen. 
Wir führten unseren Versuch jeweils mit einer Versuchsperson durch. Es wurde im allgemeinen "getaktet" gearbeitet; m.a.W., die Informanten hatten nicht alle Karten gleichzeitig zur Verfügung. Allerdings wurde, falls gewünscht, die Bearbeitung bestimmter Karten solange zurückgestellt, bis die anderen, leichter zu bearbeitenden Karten abgetestet worden waren.

\subsection{Ergebnisse des Tests}

Wir wollen nun die Ergebnisse unseres Experiments betrachten. Im folgenden haben wir die Antworten der Versuchspersonen zu jeder einzelnen lexikographischen Definition aufgeführt. Der Informantengruppe $A$ sind die Bedeutungsexplikationen aus dem Wörterbuch von OZEGOV, der Informantengruppe $B$ die aus dem EKW vorgelegt worden. Die in Klammern stehende $\mathrm{Zahl}$ hinter den verschiedenen Verben bzw. Adjektiven bezeichnet die Häufigkeit, mit der die Versuchspersonen aus der jeweils betrachteten Gruppe Wortformen des betreffenden Lexems in ihren Reduktionsparaphrasen verwendet haben.

\section{Gruppe A}

1. arestovyvat'

Policija podvergaet Petra zaključeniju pod stražu. arestovyvat' (4)

sá̃at' (2)

2. drobit'

Sgibaja ili udarjaja s siloj, Pëtr razdoljaet l'dinu na melkie časti. $\rightarrow$

razbivat'

razdrablivat'

zavieet'

Brigadir nachoditsja v podéinënnosti u načal'nika pri otsutstvii samostojatel'nosti. podăinjat'sja 
4. nabljudat'

Biolog podvergaet volkov izučeniju, osnovannomu na principach sistemy znanij o zakonomernostjach $v$ razvitii

prirody, obščestua. $\rightarrow$

izučat' (3)

nabljudat' (2)

éksperimentirovat'

5. nakazyvat'

Tovarĭ̌c̆ Lyžnikov prinimaet meru vozdejstvija protiv

tovarï̌ča Ivanova, soveršivsego prestuplenie, prostupok. -

nakazyvat' (2)

vystupat' protiv (2)

ugrožat' (1)

predupreždat' (1)

6. predlagat'

Pëtr predstavljaet Nată̌e na obsuždenie kak vozmožnost',

o kotoroj ona imeet suedenija, pojti na sobranie. $\rightarrow$

priglašat'

ne otkazyvat'

obsuždat’ (2)

7. prenebregat'

Larisa otnositsja bez sosredotočenija myslej na zdorov'e.

ne dumat' o (4)

vredit' (2)

8. provocirovat'

Gusar vyzyvaet sopernika na dejstvija, kotorye mogut

povlěc' za soboj tjažëlye dlja nego posledstvija, putëm

predatel'skogo povedenija, podstrekatel'stva. $\rightarrow$

ubivat' (4)

drat'sja (1)

provocirovat' (1)

9. razbivat'

Sgibaja ili udarjaja s siloj, Pëtr razdeljaet zerkalo na ̌asti. $\rightarrow$ 
razbivat'

10. ubeżdat'

Pëtr stavit Natašu v neobchodimost' poverit', 'cto on ne vinovat.

dokazyvat' (3)

ubě̌dat' (2)

opravdyvat'sja pered (1)

11. udarjat'

Proizvodja rezkij tolčok, učitel' prikasaetsjas siloj k stolu. $\rightarrow$

udarjat' (3)

stučat' (2)

prikasat'sjak

12. učityvat,

Chimik napravljaet sosredotočenie myslej ili zrenija na vyvody kolleg.

slüsat' (4)

izučat' (2)

13. $u \check{c} i t^{\prime} s j a$

Ponjav, Nataša sochranjaet matematiku v pamjati kak

sleduet.

zapominat' (5)

razbirat'sjav (1)

14. ostorožnyj

Predvidja, Tanja prigotovijaetsja k vozmožnoj situacii, sposobnoj vyzvat' kakoj-n. vred, gorestnoe sobytie. $\rightarrow$ cutkij (2)

mnitel'nyj (2)

cuvstvitel'nyj (1)

rasčëtlivyj (1)

15. pročnyj

Kolonna trudno prevraščajuščajasja v razvaliny. $\rightarrow$

krepkij (4)

nerazbivaemyj 
16. spravedlivyj

Tanja - dejstvujušcaja bespristrastno, $v$ sootvetstviis

tem, ¿́to suščstunet $v$ dejstvitel'nosti.

ob-ektivnyj (3)

gumannyj (2)

spravediivyj (1)

17. jasnyj

Nebo ničem ne sdelano tëmnym ili ničem tëmnym ne zakryto,

ne propuskajušcim sueta.

jasnyj (5)

cistyj (1)

Gruppe B

1. arestovyvat'

Policija v sootvetstvii s predvaritel'nym rasporjaže-

niem lišaet Petra svobody za vorovstuo.

arestovyvat' (5)

sažat' (1)

2. drobit'

Pëtr delit l'dinu udarom molota.

razbivat' (5)

razdrablivat' (1)

3. zaviset'

Načal'nik možet pričinjat' izmenenija v svojstvach, so-

stojanijach ili povedenii brigadira.

vospityvat'

(3)

Die zusätzliche Anweisung, bei der Reduktionsparaphrasierung die syntaktischen Funktionen von 'načal'nik' und

'brigadira' zu verändern (vgl. S. 195), wurde von den Versuchspersonen nur im Fall von 
podčinjat'sja (2)

stojat' podviijaniem (1) beachtet.

4. nabljudat'

Biolog sledit za povedeniem volkov s cel'ju uznat' ich svojstva.

nabljudat' (3)

izučat' (2)

issledovat'

5. nakazyvat'

Tovarǐ̌c Ly̌̃nikov pričinjaet tovarišcu Ivanovu, soveršivšemu kontrabandu, lišsenie zarplaty s cel'ju ispravit' ego.

nakazyvat' (6)

6. predlagat'.

Pëtr soob̌̌scaet Nată̌e, čto, esli ona chočet pojti na sobranie, vozmožnost' čego zavisit ot nego, to on pričinit ètu vozmožnost'. $\rightarrow$

priglašat'

predlagat'

7. prenebregat'

Larisa dejstvuet, sčitaja, čto zdorov'e nesuščestveno dija svoej dejatel'nosti.

prenebregat' (3)

ne berečs (2)

vredit' (1)

8. provocirovat'

Imeja cel'ju pričinit' soperniku uščcerb, gusar pytaetsja pričinjat' draku s ego storony, kotoraja možet posiužit' opravdaniem dlja realizacii ètoj celi.

provocirovat"

0

9. razbivat'

Udarjaja zerkalom ili po zerkalu, Pëtr delit ego. - 
razbivat,

bit' (1)

10. $\underline{u b e \check{z} \text { dat’ }^{\prime}}$

Privodja argumenty, Pëtr pytaetsja pričinjat' Natašu š́itat', čto on ne vinovat.

ubeždat' (4)

dokazyvat' (1)

zaverjat' (1)

11. udarjat'

Učitel' rezko i kratkovremenno privodit ukazku v kontakt

so storom.

stucat' (3)

udarjat, (2)

bit' (1)

12. učityvat'

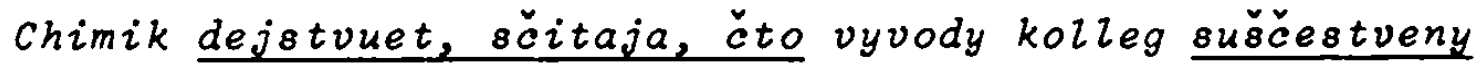
dija svoej dejatel'nosti. -

primenjat' (2)

ispol'zovat' (2)

učityvat' (2)

13. ućit'sja

Natắa, soznatel'no zatračivaja usilija, stremitsja priobresti matematiku.

zanimat'sja (2)

izučat' (2)

ǘit' (2)

14. ostorožnyj

Tane svojstvenno želanie izbegat' situacij, cotorye mogut

byt' opasnymi ili neprijatnymi dija ne $\ddot{e}_{2}$ i poètomu ona staraetsja dejstvovat' s učëtom vsech tech faktorov, kotorye, po ë̈ mneniju, mogut pričinit' takuju situaciju. ostorožnyj (5)

predusmotritel'nyj (1) 
15. pročnyj

Lampa takaja, kotoraja trudno razrušaetsja mechaničeskoj

siloj.

pročnyj

krepkij

(2)

nerazbivaemyj

16. spravedlivyj

Tanja sposobnaja pravil'no ocenit', kak sleduet dejstvovat', čtoby nikakim ljudjam ne bylo pričineno nezaslu-

žennoe zlo.

spravedlivyj (6)

17. jasnyj

Nebo - charakterizujuščeesja otsutstviem v atmosfere častic (naprimer $=0$ oblaka, pyl', dym, tuman ...', prepjatstvujušcic) vosprijatiju sveta.

pustoj (5)

cistyj (1) 


\section{AUSWERTUNG DES TESTS}

\subsection{Prinzipien der Auswertung der Testergebnisse}

Wir haben bereits festgelegt, daB wir unsere Aussagen iber die Verstehbarkeit der Definitionssprache des Wörterbuchs von OZEGOV und der SemS des EKW im Hinblick auf die Bedeutungsrelation vornehmen wollen, die zwischen einem Satz, der das Stichwort enthält, dessen lexikographische Definition wir in unseren Versuch eingebracht haben, und der entsprechenden Reduktionsparaphrase anzusetzen ist (vgl. S. $223 \mathrm{f}$. ). Es ist daher erforderlich, sofern das in einer Reduktionsparaphrase genannte Verb bzw. Adjektiv nicht mit dem gesuchten Stichwort übereinstimmt, in einer weiteren Informantenbefragung die vorliegenden Testergebnisse im einzelnen daraufhin zu untersuchen, ob zwischen beiden Sätzen eine Synonymierelation besteht.

Zu diesem Zweck befragten wir einen russischen Muttersprachler, dem wir die Sätze mit den gesuchten Stichwörtern und zu jedem dieser Sätze diejenigen Reduktionsparaphrasen vorlegten, in denen die Versuchspersonen Lexeme verwendet hatten, die mit den gesuchten Stichwörtern nicht identisch waren. 53 Wir wiesen unseren Informanten an, für jedes einzelne Satzpaar zu entscheiden, ob es sich um synonymische oder nichtsynonymische Sätze handelt, wobei stilistische Besonderheiten des Wortgebrauchs unberücksichtigt gelassen werden sollten. Jedes Satzpar, das dem Informanten zur Beurteilung vorgelegt wurde, bildet für das jeweils gesuchte Stichwort bzw. die Lexeme, auf die seine lexikographische Definition von den Versuchspersonen reduziert worden war, einen invarianten Kontext, da wir für die lexikalische Auffiullung ihrer syntaktischen Umgebungen die gleichen Lexeme auswählten, die wir auch bei der Konstruktion unseres Testmaterials verwendet hatten; vgl. beispielsweise die folgenden Satzpaare, in denen es sich bei (3) um die erwartete Reduktionsparaphrase der lexikographischen Definition von učityvat' (EKW) und bei (3') bzw. (3") um die im Test genannten Reduktionsparaphra- 
sen handelt, in denen Verben verwendet wurden, die mit dem gesuchten stichwort nicht identisch sind:

(3) Chimik učityvaet vyvody kolleg. $\rightarrow$

(3') Chimik primenjaet vyvody kolleg.

(3") Chimik izpol'zuet vyvody kolleg.

Wir hielten es für erforderlich, unseren Informanten die gesuchten Stichwörter und die entsprechenden Lexeme, die in den Reduktionsparaphrasen genannt worden waren, hinsichtlich inrer Bedeutungsrelation in den oben genannten invarianten Kontexten klassifizieren zu lassen, um die Möglichkeit auszuschließen, daß unterschiedliche lexikalische Umgebungen den Informanten veranlassen, sein Urteil über das Vorhandensein von Synonymie bzw. Nichtsynonymie zwischen zwei gegebenen Sätzen von den kombinatorischen Eiqenschaften der betrachteten Lexeme abhängig zu machen.

Unsere Entscheidung, nicht auf Synonymiewörterbijcher als Hilfsmittel zurückzugreifen, um die Bedeutungsrelation zwischen den Teilsätzen der Satzpaare festzustellen, sondern eine Informantenbefragung vorzunehmen, liegt in erster Linie in den Schwierigkeiten begründet, die der Versuch mit sich bringt, den Synonymiebegriff zu definieren. Die lexikographische Praxis der gängigen russischen Synonymiewörterbücher besteht darin, zu einem Ausgangswort u.a. eine sogenannte synonymische Reihe oder eine synonymische Gruppe (sinonimičeski, rjad, sinonimičeskaja gruppa) von Lexemen anzuführen und die Bedeutungsunterschiede zu spezifizieren, durch die sich diese Lexeme untereinander und relativ zum Ausgangswort auszeichnen (EVGEN'EVA 1970, 15ff.; 1975, 6ff.). Es ist leicht einzusehen, daß es für uns äuBerst schwierig ist, in Hinsicht auf Lexeme synonymischer Reihen zu entscheiden, ob es ihre relativen Bedeutungsunterschiede überhaupt noch zulassen, sie als Synonyme im engeren oder weiteren Sinne zu bezeichnen. ${ }^{54}$ Die Problematik des Synonymiebegriffs besteht eben darin, daB er eine Relation 2 wischen den Bedeutungen sprachlicher Einheiten bezeichnet, deren Beurteilung individuell von Sprecher zu Sprecher variiert und dementsprechend in unterschiedlichen 
Ausprägungen angesetzt werden kann (MEL'CuK 1974b, 10, 46 sowie S. $72 \mathrm{ff}$. der vorliegenden Arbeit).

Unsere Schwierigkeiten lassen sich auch nicht dadurch lösen, daB wir uns auf das Konzept lexikalischer Synonymie berufen, das die Konstrukteure des MST entwickeln. Gemäß dieser Auffassung lassen sich Lexeme, zwischen denen die Sinnrelation der Synonymie anzusetzen ist, in Synonyme im strengen Sinne (kurz: Synonyme) oder Synonyme im weiteren Sinne (kurz: Quasisynonyme) einteilen. Zwei gegebene Lexeme werden im MST als Synonyme angesehen, wenn sie den folgenden beiden Bedingungen genügen:

"... die Wörter $X$ und $Y$ heiBen Synonyme, wenn 1$) X$ und $Y$ synonymisch sind, d.h. ' $X{ }^{\prime}={ }^{\prime} y$ ' ist, und 2 ) bei $X$ und $Y$ die syntaktischen klassen ( $\approx$ Wortartzugehörigkeit) sowie ihre syntaktischen Valenzen identisch sind ..."

Im Gegensatz dazu definieren die Konstrukteure des MST zwei gegebene Lexeme als Quasisynonyme, wenn deren Bedeutungsexplikationen in wesentlichen Teilen identisch sind:

"Ausdruicke mit Bedeutungen, die noch so nahe (verwandt, teilwe ise deckungsgleich), aber dennoch nicht identisch sind, diurfen nicht als synonymisch im strengen Sinne des Terminus angesehen werden ... Wenn wir zwischen den Bezeichneten zweier sprachlicher Einheiten auch nur den geringsten Bedeutungsunterschied aufzeigen können, so sind diese Einheiten nicht synonymisch ... Solche Paare (oder Reihen) bezeichnen wir als Q u a $s$ i s y $n$ o $n$ y m e (engl. near-synonyms) ..." (MEL'CUK 1974b, 47)

"Quasisynonyme unterscheiden sich von genauen Synonymen hinsichtlich des ersten Merkmals: Ihre Explikationen haben einen groBen ... gemeinsamen Teil, sie sind jedoch nicht völlig kongruent." (APRESJAN 1974b, 235)

Die Auffassung von lexikalischer Synonymie, die die Konstrukteure des MST vertreten, bringt den unbestreitbaren Vorteil mit sich, bei der semantischen Beschreibung lexikalischer Einheiten auf die Sinnrelation der Synonymie nicht weitgehend verzichten zu müssen, da sich im Wortschatz einer Sprache 
kaum zahlreiche Lexeme finden lassen dürten, die als Synonyme (im engeren Sinne) angesehen werden können. AuBerdem macht sie den wenig präzisen Begriff der Bedeutungsnuance (smyslovoj ottenok, ottenok značenija) iberfliussig, der in der Lexikographie sehr häufig in Verbindung mit dem strengen Synonymiebegriff verwendet wird. Dabei führt die mangelnde Klarheit des Begriffs der Bedeutungsnuance dazu, den strengen Synonymiebegriff völlig auszuhöhlen, weil unter Bedeutungsnuance entweder Eigenschaften von Lexemen verstanden werden, die deren lexikalischer Bedeutung bzw. Konnotation oder deren Kombinierbarkeit mit anderen Lexemen zugewiesen werden können (APRESJAN 1974b, 243-248).

Dem Vorteil, den die Konzeption lexikalischer Synonymie im MST für die semantische Beschreibung bietet, steht das Problem der Abgrenzung von Synonymen und Quasisynonymen gegenuber. Es bleibt der Intuition des Analysators uber die Bedeutungen zweier gegebener Lexeme vorbehalten, ob er zwischen ihnen die Relation der Synonymie im engeren bzw. im weiteren Sinne oder uberhaupt keine Synonymierelation ansetzt.

Vor den gleichen Abgrenzungsschwierigkeiten stünden wir bzw. unser Informant, wenn wir zur Evaluierung unserer Testergebnisse die Auffassung von lexikalischer Synonymie im MST heranziehen wïrden. Aus diesem Grunde stellten wir unserem Informanten lediglich eine binäre Bewertungsmöglichkeit zur Verfiugung, um die Bedeutungsrelation zwischen den Sätzen mit dem jeweils gesuchten Stichwort und den Reduktionsparaphrasen, in denen unsere Versuchspersonen Verben bzw. Adjektive genannt hatten, die nicht mit dem jeweils gesuchten Lexem ibereinstimmen, zu beurteilen. 
8.2 Bewertung der Reduktionsparaphrasen hinsichtlich ihrer Synonymie

Wir wollen nun betrachten, wie unser Informant die Testergebnisse, die er zu beurteilen hatte, klassifiziert hat. Dazu führen wir parweise die Sätze mit den gesuchten Stichwörtern und die entsprechenden Reduktionsparaphrasen an. Ein $(+)$ hinter einem Satzpaar gibt an, daß unser Informant die betrachteten Sätze als synonymisch beurteilt hat, ein (-) bedeutet, daB er sie als nichtsynonymisch eingestuft hat. Unter $A$. ist die Bewertung der Testergebnisse für die lexikographischen Definitionen aus dem Wörterbuch von OZ̈EGOV aufgeführt, unter B. die der Testergebnisse für die lexikographischen Definitionen aus dem EKW.

A.

1. arestovyvat'

(1) Policija arestovyvaet Petra.

(1') Policija sažaet Petra. (+)

2. drobit'
(1) Pëtr drobit l'dinu.
(1') Pëtr razdrablivaet l'dinu. (+)
(1") Pëtr razbivaet $l^{\prime}$ dinu. (-)

3. nabljudat'
(1) Biolog nabljudaet volkov. $\rightarrow$
(1') Biolog izuçaet volkov. (+)
(1") Biolog èksperimentiruet s volkami. (-)

4. nakazyvat'
(1) Tovarǐ̌č Ly̌̌nikov nakazyvaet tovarišča Ivanova. $\rightarrow$
(1') Tovarišč Ly̌̌nikov vystupaet protiv tovarišča Ivanova. (-)
(1") Tovarišc̆ Lyžnikov ugrö̌aet tovarišču Ivanovu. (-)
(1"') Tovarišč Lyžnikov predupreždaet tovarišča Ivanova. (1) 
5. predlagat'

(1) Pëtr predlagaet Nataše pojti na sobranie. $\rightarrow$

(1') Pëtr ne otkazyvaet Nataše pojti na sobranie. (-)

(1") Pëtr obsuždaet s Natašej pojti na sobranie. (-)

(1"') Pëtr priglašaet Natašu pojti na sobranie. (+)

6. prenebregat'

(1) Larisa prenebregaet zdorov'em.

(1') Larisa ne dumaet o zdorov'e. (+)

(1") Larisa vredit zdorov'ju. (+)

7. provocirovat'

(1) Gusar provociruet sopernika. $\rightarrow$

(1') Gusar ubivaet sopernika. (-)

(1") Gusar derëtsja s sopernikom.(-)

8. ubezdat'

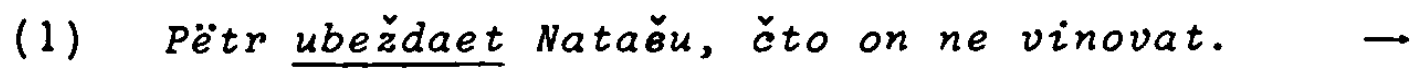

(1') Pëtr dokazyvaet Nataše, čto on ne vinovat. (-)

(1") Pëtr opravdyvaetsja pered Natašej, čto on ne vinovat. $(-)$

9. udarjat'

(1) Učitel' udarjaet po stolu. $\rightarrow$

(1') Učitel' prikasaetsja k stolu. (-)

(1") Učitel' stučit po stolu. (+)

10. učityvat'

(1) Chimik učityvaet vyvody kolleg.

(1') Chimik izučaet vyvody kolleg. (-)

(1") Chimik slušaet vyvody kolleg. (-)

11. $\frac{u c i t ' s j a}{(1)}$

(1) Nataša učitsja matematike. $\rightarrow$

(1') Nataša zapominaet matematiku. (-)

(1") Nată̌a razbiraetsja $v$ matematike. (-)

12. zaviset'

(1) Brigadir zavisit ot načal'nika. $\rightarrow$

(1') Brigadir podčinjaetsja načal'niku. (-) 
13. ostorǒ̌nyj

(1) Tanja ostorožnaja. $\rightarrow$

(1') Tanja čutkaja. (-)

(1") Tanja mnitel'naja. (-)

(1"') Tanja rašcëtlivaja. (-)

(1" ") Tanja čuvstvitel'naja. (-)

14. pročnyj

(1) Kozonna pročnaja. $\rightarrow$

(1') KoZonna krepkaja. (+)

(1") KoZonna nerazbivaemaja. (+)

15. spravedlivyj

(1) Tanja spravedlivaja.

(1') Tanja gumannaja. (-)

(1") Tanja ob-ektivnaja. (-)

16. jasnyj

(1) Nebo jasnoe. $\rightarrow$

(1') Nebo ćistoe. (+)

B.

1. arestovyvat'

(1) Policija arestovyvaet Petra za vorovstvo. $\rightarrow$

(1') Policija sažaet Petra za vorovstvo. (+)

2. drobit'

(1) Pëtr drobit l'dinu. $\rightarrow$

(1') Pëtr razdrablivaet 2'dinu. (+)

(1") Pëtr razbivaet 2 'dinu. (-)

3. zaviset'

(1) Brigadir zavisit ot načal'nika. $\rightarrow$

(1') Brigadir podčinjaetsja načal'niku. (-)

(1") Brigadir stoit pod vlijaniem načal'nika. (-)

(1"') Načal'nik vospityvaet brigadira. (-) 
4. nabljudat'

(1) Biolog nabljudaet volkov. $\rightarrow$

(1') Biolog izučaet volkov. (+)

(1") Biolog issleduet volkov. (+)

5. predlagat'

(1) Pëtr predlagaet Nataše pojti na sobranie. -

(1') Pëtr priglašaet Natašu pojti na sobranie. (+)

6. prenebregat'

(1) Larisa prenebregaet zdorov'em. $\rightarrow$

(1') Larisa ne berežët zdorov'e. (+)

(1") Larisa vredit zdorov'ju. (+)

7. razbivat'

(1) Pëtr razbivaet zerkalo. $\rightarrow$

(1') Pëtr b’ët zerkalo. (+)

8. $u b e \check{z} d a t^{\prime}$

(1) Pëtr ubě̌daet Natašu, čto on ne vinovat. $\rightarrow$

(1') Pëtr dokazyvaet Nataše, čto on ne vinovat. (-)

(1") Pëtr zaverjaet Nataše, čto on ne vinovat. (-)

9. udarjat'

(1) Učitel' udarjaet ukazkoj po stolu. $\rightarrow$

(1') Učicel' stučit ukazkoj po stolu. (+)

(1") Učitel' b'ët ukazkoj po stolu. (+)

10. učityvat'

(1) Chimik učityvaet vyvody kolleg. $\rightarrow$

(1') Chimik primenjaet vyvody kolleg. (+)

(1") Chimik ispol'zuet vyvody kolleg. $(+)$

11. $\underline{\text { ucit'sja }}$

(1) Nată̌a učitsja matematike. $\rightarrow$

(1') Nataša zanimaetsja matematikoj. (+)

(1") Natǎ̌a izučaet matematiku. (+)

(1"') Nataša učit matematiku. (+) 
12. ostorožnyj
(1) Tanja ostorožnaja.
(1') Tanja predusmotritel'naja. (+)

12. pročnyj
(1) Kolonna pročnaja.
(1') Kolonna krepkaja. (+)
(1") Kolonna nerazbivaemaja. (+)

14. jasnyj
(1) Nebo jasnoe.
(1') Nebo pustoe. (-)
(1") Nebo cistoe. (+)
8.2.1 Besprechung der Synonymierelation zwischen einigen aus- gewählten Reduktionsparaphrasen unter stilistischen, morpho-syntaktischen und semantischen Aspekten

AufschluBreich sowohl für die Durchführung unseres Experiments als auch für die semantische Beschreibung einiger der oben genannten Lexeme sind bestimmte Kommentare, die unser Informant während der Befragung zu mehreren seiner Klassifikationsentscheidungen abgab.

Er bezeichnete beispielsweise im Hinblick auf die unterschiedlichen stilistischen Werte der Lexeme, die in den Reduktionsparaphrasen genannt worden waren, lediglich das Verb sažat' (vgl. S. 225, 228) als ein Lexem, das nicht einer standardsprachlichen Stilnorm entspreche. Die große Homogenität, die die in den Reduktionparaphrasen verwendeten Lexeme bezüglich ihres stilistischen Wertes aufweisen, legt die Vermutung nahe, daß die Versuchspersonen, obwohl sie zweifellos auch über nichtstandardsprachliche lexikalische Entsprechungen verfügten, wahrscheinlich durch Situation und Umstände des Experiments dazu veranlaßt wurden, in den Reduktionsparaphrasen nur standardsprachliche Stilregister zu verwenden.

In zwei fällen bezweifelte unser Informant die morpho-syntaktische Wohlgeformtheit von Sätzen, die er zu klassifizieren 
hatte. Die Satzpare

(1) Biolog nabljudaet volkov.

(1') Biolog izuçaet / issleduet volkov. (vgl. S. 236, 239)

stufte er als zumindest stilistisch nicht wohlgeformt ein.

Er verbesserte die Sätze durch beispielsweise

(2) Biolog nabljudaet (izuçaet, issleduet) $\check{i} i z n^{\prime}$ volkov.

Das Urteil unseres Informanten wird zumindest im Hinblick auf issledovat" ebenfalls durch das "Učebnyj slovar' sočetaemosti slov russkogo jazyka" gestijtzt, da auch dort keine Wendung mit kogo angegeben wird (Učebnyj slovar' 1978, 213).

Den Satz

(3) Pëtr opravdyvaetsja pered Natašej, čto on ne vinovat.

(vgl. S. 237) bezeichnete er als grammatisch nichtwohlgeformt, da das verb opravdyvat'sja nicht mit einem Komplementsatz verwendet werden durfe.

Die Kommentierung, die unser Informant zur stilistischen (-grammatischen) Wohlgeformtheit von (1) und (I') gab, wirft ein interessantes Licht auf die Problematik, die linguistische Beurteilungen von Wohlgeformtheit bzw. Normverletzungen mit sich bringen. 55 Es ist nicht ausgeschlossen, daß die Selektionsrestriktionen, denen die Realisierungsmöglichkeiten der objektphrase bei nabljudat', izučat' und issledovat' (in der hier betrachteten Bedeutung) unterliegen, von Sprechern individuell verschieden behandelt werden. Sollten weitere Untersuchungen diese Vermutung erhärten, so müBte eine angemessene semantische Beschreibung der Verben diesem Umstand Rechnung tragen, indem sie die Beschränkungen der semantisch-lexikalischen Kombinierbarkeit der Lexeme näher spezifiziert. Sie müBte die Bedingungen angeben, unter denen bei nabljudat', izučat' und issledovat' (in der hier betrachteten Bedeutung) die Objektphrase durch Bezeichnungen für Lebewesen und/oder durch Bezeichnungen für Eigenschaften von Lebewesen realisiert werden können.

Im Hinblick auf die Durchführung unseres Experiments scheint das Urteil, das unser Informant uber die Wohlgeformtheit von 
(1) und (1') abgegeben hat, allerdings keine hinreichende Berechtigung für die Vermutung zu sein, daB wir das Testmaterial fehlerhaft, d.h. Zwangsläufig zu grammatischen Abweichungen führend, konstruiert haben. Die Tatsache, daB alle 12 Versuchspersonen die Wortverbindungen nabljudat' (izucat', issledovat') volkov in ihren Reduktionsparaphrasen verwendet haben, spricht dagegen. Die Aussagen, die unser Informant zur Wohlgeformtheit von (3) gemacht hat, können wir weder hinsichtlich der Durchführung unseres Versuchs noch bezüglich anderer linguistischer Fragestellungen bewerten, da die Wortverbindung opravdyvat'sja pered kem-n., ćto ... in nur einer einzigen Reduktionsparaphrase benutzt worden ist.

Interessant unter dem Gesichtspunkt der semantischen Beschreibung von predlagat' und priglasat, (in der hier betrachteten Bedeutung) sind die Vorbehalte, unter denen unser Informant die Synonymierelation zwischen

(4) Pëtr predlagaet Natăse pojti na sobranie.

und

(4') Pëtr priglašaet Natašu pojti na sobranie.

(vgl. S. 237, 239) gelten lassen wollte. Er begründete sein Urteil, beide Sätze als synonymisch anzusehen, mit dem Vorhandensein der Wortverbindung na sobranie in den durch pojti eingeleiteten Komplementsätzen bei predlagat, und priglašat'. Eine Substitution des Syntagmas na sobranie durch Wortverbindungen wie beispielsweise $v$ kino, $v$ teatr, na tancy, $v$ restoran, na stadion, popit' kofe u.ä. würde dagegen unseren Informanten dazu veranlassen, die Sätze als nichtsynonymisch einzustufen. Den Bedeutungsunterschied, der beispielsweise zwischen

(5) Pëtr predlagaet Natăse pojti v kino.

und

(5') Pëtr priglǎsaet Nată̌u pojti v kino.

anzusetzen ist, erblickte unser Informant in der Tatsache, daß bei der Verwendung von priglašat' der Mitspieler in Agensfunktion sich verpflichtet, materielle Aufwendungen auf sich zu nehmen, die sowohl für inn als auchlaudententigt 9783954792764 
spieler in Adressatenfunktion entstehen, wenn beide bestimmte Handlungen, wie sie beispielsweise durch pojti $v$ kino bezeichnet werden, gemeinsam ausführen. Bei der Verwendung von predlagat' geht der Mitspieler in Agensfunktion eine derartige 0 bligation nicht ein. 56

Diese Feststellung unseres Informanten ist äußerst aufschluBreich im Hinblick auf die lexikographischen Definitionen, die das EKW und das wörterbuch von OZEGOV den oben genannten Verben zuweisen; vgl. die Bedeutungsexplikationen (6) $-(9)$ :

(6) predlagat' (EKW):

la) $X$ predlagaet $Y z-u=' X$ soobščaet $Z-u$, čto, esli $Z$ chočet, čtoby $X$ proizvodil dejstvie $Y$, to $X$ budet proizvodit' dejstvie $Y$.'

1b) $X$ predlagaet $Y z-u=' X$ soobščaet $z-u$, čto, esli $z$ chočet proizvodit' dejstvie $Y$, vozmožnost' čego zavisit ot $x-a$, to $x$ kauziruet ètu vozmožnost'.'

Chozjain predložil gostju ostat'sja perenočevat'. (VOL'F 19:

2a) X predlagaet $Y z-u=' X$ soob ščaet $Z-u$, čto X sčitaet, čto budet chorošo, esli budet imet' mesto situacija $Y$, osuščestulenie kotoroj a avisit ot $z-a .^{\prime}$

Predlagaju, čtoby nă̌a junaja gost'ja rasskazala nam interesnuju skazku. (VOL'F 1973, 5)

2b) $X$ predlagaet $Y z-u=' X$ predlagaet $2 a$ Z-u, čtoby 2 vypolnil trebovanie $2 a x-a$ sdelat'y.'

Predlagaju Vam javit'sja $v$ sud. (VOL'F 1973,7 ) 
(3) $X$ predlagaet $y z-u=' X$ sozdal informacionnyj ob-ekt $y$ i soobščaet ob $Y-e$ Z-u, kotoryj možet ispol'zovat' $Y$.'

Professor Charris predlagaet nam novyj metod postanovki éksperimenta. (VOL'F 1973, 8)

(7) predlagat' (OZEGOV):

predlagat' $=1$. 'Predstavljat' na obsuždenie kak izvestnuju vozmožnost" "

Predlagat' postroit' dom

2. 'Sprašivat', zadavat'"

Predlagat' vopros

3. 'Predostavljat' $v \check{c}$ ' $\ddot{e}-n$. rasporjaženie' Predlagat' svoi uslugi

4. 'Trebovat', predpisyvat' čto-n. sdelat"' Predlagat' zakončit' rabotu $v$ nedel'nyj srok (OZEGOV 1973,532)

(8) priglašat’ (EKW):

$X$ priglašaet $y-a$ delat' $Z(v W)=$ ' $X$ prosit 1 ili predlagaet $2 Y-u$, ¿́toby $Y$ proizvodil dejstvie 2 (v W, čto svjazano $s$ peremeščeniem $y-a), i$ $X$ soobščaet $y-u$, čto kauziruet vozmožnost' togo, čtoby y delaz z.'

Chozjaeva priglašajut nas na obed, v restoran. (VOL'F 1973,17-19)

(9) priglašsat' (OŽEGOV):

priglašat' $=1$. 'Prosit' prijti' Priglasat' na zasedanie

2. 'Prosit' vypolnjat' kakuju-n. rabotu, 


\section{poručenie' \\ Priglašat’ na službu (OŽEGOV 1973, 541)}

Betrachten wir die oben genannten lexikographischen Definitionen, so zeigt sich, daB weder das Wörterbuch von OZEGOV noch das EKW die semantische Besonderheit vermerken, durch die sich nach der Auffassung unseres Informanten priglašat' und predlagat, in bestimmten Kontexten unterscheiden. 57 Die verschiedenen Bedeutungsexplikationen, die das EKW dem polysemen Verb predlagat' und dem monosemen Verb priglašat' zuweist, enthalten zwar partiell Teilexplikationen, in denen angegeben wird, daß bei diesen Stichwörtern der Mitspieler in Agensfunktion im Hinblick auf den Mitspieler in Adressatenfunktion die Obligation zu einer Handlung eingeht. Diese sind jedoch nicht hinreichend bezüglich des oben genannten Bedeutungsunterschieds zwischen predzagat' und priglašat' in bestimmten Kontexten differenziert.

Es bleibt der zukünftigen Forschung in der linguistischen Semantik überlassen, herauszufinden, ob der Bedeutungsunterschied, den unser Informant zwischen den beiden Lexemen angesetzt hat, aufrechterhalten werden kann. Von der Lösung dieser Frage wird die Entscheidung abhängen, ob die spezifischen Verwendungsbedingungen von priglašat, als Ambiguitäts-oder als Vagheitsphänomene behandelt werden müssen (vgl. S. $54 \mathrm{f.}$ ). Eine Bewertung der Güte der lexikographischen Definitionen, die das EKW fiur predlagat' und priglasat' gibt, muB ebenfalls von der Beantwortung dieser Frage abhängig gemacht werden.

\section{3 Auswertung der Testergebnisse}

Wir können nun dazu übergehen, Aussagen über die lexikographische Verwendbarkeit der SemS des EKW zu machen. Zu diesem Zweck metrisieren wir die vorliegenden Testergebnisse bezüglich des Vorhandenseins einer Synonymie- bzw. einer Nichtsynonymierelation $z$ wischen den verschiedenen Reduk- 
tionsparaphrasen, die die Versuchspersonen in unserem Experiment genannt haben, und den entsprechenden Sätzen mit dem jeweils gesuchten Stichwort. Wir bedienen uns dabei einer binären Bewertungsskala, indem wir synonymischen Satzpaaren einen Punkt und nichtsynonymischen Satzpaaren Null Punkte zuweisen. Da wir zu jeder der 17 lexikographischen Definitionen des Wörterbuchs von OZEGOV und des EKW, jeweils 6 Versuchspersonen befragt haben, konnten im Idealfall $17 \times 6=102$ Punkte erzielt werden.

Um festzustellen, inwieweit im Vergleich zum Idealfall totaler Verstehbarkeit der beiden getesteten Definitionssprachen diese Eigenschaft in unseren Testergebnissen faktisch ausgeprägt ist, beziehen wir die Punktzahlen, die die beiden Informantengruppen bei der Befragung zu den lexikographischen Definitionen des Wörterbuchs von OZZEGOV und denen des EKW erreicht haben auf den Maximalwert von 102 Punkten. Bezogen auf die uns zur Verfugung stehende Datenbasis gelangen wir auf diese Weise zu einer quantitativen Spezifizierung der tatsächlichen Verstehbarkeit derjenigen Ausschnitte der Definitionssprachen des Wörterbuchs von OZEGOV und des EKW, die wir in unserem Versuch untersucht haben. Oie Metrisierung erlaubt es uns, empirisch abgesicherte und somit nicht nur rein subjektiv begruindete Vermutungen iber die lexikographische Verwendbarkeit der Sems des EKW relativ zur Definitionssprache des Wörterbuchs von oZEGOV anzustellen.

Wir wollen nun unsere Testergebnisse gemä3 dem oben genannten Punktesystem metrisieren. Dazu fihren wir die Stichwörter an, deren lexikographische Definition Gegenstand unseres Experiments waren, sowie die entsprechenden Lexeme, die die Versuchspersonen in ihren Reduktionsparaphrasen verwendet haben. Hinter den Lexemen wird in runden Klammern die Häufigkeit angegeben, mit der die Wörter in den Reduktionsparaphrasen genannt worden sind. In Schrägstrichen werden zu jedem einzelnen Lexem diejenigen Punkte angefuhrt, die je nachdem, ob Synonymie oder Nichtsynonymie mit dem gesuchten Stichwort besteht, erzielt worden sind. Oiese Punk- 
te werden für jedes Stichwort addiert.

Gruppe A. (OZEGOV):

1. arestovyvat' arestovyvat' sažat'

2. drobit' razbivat'

razdrablivat'

3. zaviset' podčinjat'sja

4. nabljudat'

izučat'

iss ledovat'

eksperimentirovat,

5. nakazyvat'

nakazyvat'

vystupat' protiv

ugrožat"

predupreždat'

6. predlagat'

ne otkazyvat'

obsuždat'

7. prenebregat'

ne dumat' 0

vredit'

8. provocirovat"

ubivat'

drat'sja

provocirovat'
(3) $/ 3 /$

(4) /4/

(2) $1 \frac{21}{6}$

(4) $/ 0 /$

(2) $1 \frac{21}{2}$

(6) $10 /$

(2) $12 /$

(1) $1 \frac{0}{5}$

(2) $/ 21$

(2) $10 /$

(1) $10 /$

(1) $10 /$

(2) 121

(2) $10 /$

(2) $10 /$

(4) $/ 4 /$

(2) $1 \frac{21}{6}$

(4) $10 /$

(1) $10 /$

(1) $/ \frac{1}{1}$
9. razbivat'

(6) $/ \frac{6 /}{6}$

10. ubeždat'

dokazyvat' (3) $/ 0 /$

ubeždat'

(2) $/ 21$

opravdyvat'sja

pered

(1) $10 /$

11. $\frac{\text { udarjat' }}{\text { udarjat' }}$

(3) $/ 3 /$

stučat'

(2) 121

prikasat'sja $k$

(1) $1 \frac{0 /}{5}$

12. $\frac{u c ̌ i t y v a t ’}{\text { slušat' }}$

izučat'

(4) $10 /$

(2) $10 /$

13. $u \check{c} i t^{\prime} s j a$

zapominat' (5) $/ 0 /$

razbirat'sja v

(1) $10 /$

14. ostorožnyj
čutkij
(2) $10 /$
mnitel'nyj
(2) $10 /$
čuvstvitel'nyj
(1) $10 /$
rasčëtlivyj
(1) $1 \frac{10 /}{0}$

15. pročnyj

krepkij (4) /4/

nerazbivaemyj

(2) $1 \frac{21}{6}$

16. spravedlivyj

ob-ektivnyj (3) /0/

gumannyj

(2) $10 /$

spravedlivyj 
17. jasnyj
jasny $j$
(5) $/ 5 /$
čistyj
(1) $/ \frac{1}{6}$

Gesamtzahl der erreichten Punkte: 50

Gruppe B. (EKW):

1. arestovyvat' arestovyvat, sažat,

2. drobit'

razbivat'

razdrablivat"

3. zaviset'

vospityvat'

podčinjat'sja

stojat' pod

vijianiem

4. nabljudat'

nabljudat'

izučat,

issledovat'

5. nakazyvat'

nakazyvat'

6. predlagat'

prediagat"

priglašat,

7. prenebregat'

prenebregat'

ne berečs

vredit'
(5) $/ 5 /$

(1) $/ \frac{1}{6}$

(5) $10 /$

(1) $/ \frac{1}{1}$

(3) $10 /$

(2) $10 /$

(1) $/ \frac{1}{I}$

(3) $/ 3 /$

(2) $/ 2 /$

(1) $/ \frac{1}{6} /$

(6) $1 \frac{6}{6}$

(4) $14 /$

(2) $1 \frac{21}{6}$

(3) $/ 3 /$

(2) 121

(1) $/ \frac{1}{6}$
8. provocirovat'

provocirovat'

(5) $/ 5 /$

$\emptyset$

(1) $\frac{10 /}{5}$

9. razbivat,

razbivat,

bit

(5) $/ 5 /$

(1) $/ \frac{1}{6}$

10. $\frac{u b e \check{z} d a t^{\prime}}{u b e \check{z} d a t^{\prime}}$

dokazyvat'

(4) $14 /$

zaverjat"

(1) $10 /$

(1) $1 \frac{1}{4}$

11. udarjat'

(3) $/ 3 /$

udarjat,

(2) $/ 21$

bit'

(1) $/ \frac{1}{6}$

12. ucityvat

primenjat, (2) /2/

ispol'zovat' (2) /2/

učityvat.

(2) $1 \frac{21}{6}$

13. ucit'sja

zanimat'sja (2) /2/

izučat'

(2) 121

učit'

(2) $\frac{121}{6}$

14. ostorožnyj

ostorožnyj

(5) $/ 5 /$

predusmotritels

nyj

(1) $/ \frac{1}{6}$ 
15. pročnyj

pročnyj

krepkij

nerazbivaemyj
(3) $/ 3 /$

(2) 121

(1) $/ \frac{1}{6}$
17. jasnyj

pustoj

čistyj
(5) $10 /$

(1) $/ 1 /$

16. spravediivyj

spravedlivyj

(6) $1 \frac{6}{6}$

Gesamtzahl der erreichten Punkte: 84

Beziehen wir die Punktzahlen, die unsere beiden Informantengruppen erzielt haben, auf den Maximalwert von 102 Punkten, die in unserem Versuch im Idealfall totaler (= 100 \%iger) Verstehbarkeit der von uns getesteten Fragmente beider Definitionssprachen hätte erreicht werden können, so ergibt sich für den Ausschnitt aus der Definitionssprache des Wörterbuchs von OZEGOV eine Verstehbarkeit von $49,02 \%$ und für den Ausschnitt aus der SemS des EKW eine Verstehbarkeit von 82,36\%. Die beiden Werte zeigen, daß sich die Definitionssprache des Wörterbuchs von OZEGOV und die SemS des EKW in dem AusmaB, wie beide Gegenstand unseres Experiments waren, hinsichtlich der Eigenschaft ihrer Verstehbarkeit (für Wörterbuchbenutzer) unterscheiden. Da wir unser Experiment lediglich als eine Vorausanalyse verstehen (vgl. S. 219), können wir nicht sagen, ob es sich dabei um einen signifikanten Unterschied handelt. Allerdings geben die Ergebnisse unseres Tests $z u$ der begründeten Vermutung $A n l a B$, daB die SemS des EKW bezuglich ihrer Eignung für Bedeutungsexplikationen in einem erklärenden Wörterbuch mit der Definitionssprache des Wörterbuchs von OZEGOV durchaus konkurrieren kann. Unsere positive Beurteilung der lexikographischen Eignung der SemS des EKW steht selbstverständlich unter dem Vorbehalt, daB die zukünftige linguistische Forschung durch empirische Untersuchungen, die über den bescheidenen Rahmen unserer Vorfelduntersuchung hinausgehen, noch zu zeigen hat, inwieweit unsere Einschätzung aufrechterhalten werden kann. 
8.3.1 Interpretation einiger ausgewählter Testergebnisse unter deskriptivem Aspekt

Bevor wir eine abschlieBende Bewertung der SemS des EKW im Hinblick auf den Anspruch vornehmen, den ihre konstrukteure für sie geltend machen, wollen wir einige der oben genannten Testergebnisse näher untersuchen, da sie für die lexikographische Beschreibung der entsprechenden Stichwörter, aber auch prinzipiell fur die semantische Deskription aufschluBreich sind.

Bei den lexikographischen Definitionen des EKW, zu denen wir die Informanten in unserem Experiment befragt haben, fallen die Explikationen bestimmter Stichwörter durch die geringere Punktzahl, die die Versuchspersonen bei ihrer Reduktionsparaphrasierung erzielten, deutlich auf. Es handelt sich um die lexikographischen Definitionen der Lexeme drobit' (1 Punkt), zaviset' (1 Punkt) und jasnyj (1 Punkt). Wir wollen die Bedeutungsexplikationen dieser Stichwörter nacheinander betrachten und Annahmen über die Gründe für ihr schlechtes Abschneiden im Test formulieren.

Dem Verb drobit' weist das EKW die lexikographische Definition (1) zu:

(1) $A$ drobit $B X$-om $=$ 'A delit tvërdyj $i$ pročnyj $B$ udarom instrumenta $X . .^{\prime}$ (APRESJAN 1974b, 105)

Analog zur Bedeutungsexplikation von rubit' (APRESJAN 1974b, 63) erachtet APRESJAN die Bedeutungskomponente 'udarom instrumenta' als paradigmatisch für die lexikalische Bedeutung von drobit'. Mit dieser semantischen Beschreibung stellt er sich in Gegensatz zur Beschreibung des Wörterbuchs von OZEGOV. Dort wird als paradigmatisch fü die lexikalische Bedeutung von drobit, die Tatsache angesehen, daB die Zerteilung des Mitspielers, dem in der durch drobit, bezeichneten Situation die objektrolle zukommt, im Gegensatz zu kolot' und rubit' in kleine ('melkie') Teile erfolgt; vgl. die lexikographische Definition (2): 
(2) $\underline{\text { drobit' }}=$ 'Razbivat' na melkie časti' (OZEGOV 1973, 165)

Unsere Testergebnisse (drobit, (EKW) 1 Punkt, drobit, (OZEGOV) 2 Punkte) legen die Vermutung nahe, daB eine Explikation des Lexems, die jeweils nur eine der beiden Bedeutungskomponenten 'udarom instrumenta' bzw. 'na melkie časti' als paradigmatisch für seine Bedeutung ansieht, unangemessen ist. Es scheint vielmehr der fall zu sein, daß beide seme in eine lexikographische Definition von drobit, aufgenommen werden müssen, um eine im Vergleich zu den oben genannten Explikationen angemessenere Beschreibung dessen lexikalischer Bedeutung zu erzielen.

Während wir für das schlechte Testergebnis der Bedeutungsexplikation, die das EKW und das Wörterbuch von OZEGOV für drobit' geben, wahrscheinlich das Fehlen bestimmter Bedeutungskomponenten in den Definitionen verantwortlich machen können, scheint die geringe Punktzahl, welche unsere Versuchspersonen bei der lexikographischen Definition von zaviset, (EKW) erreicht haben, eher auf bestimmte strukturelle Eigenschaften der Explikation zurückzuführen sein. Dem Verb zaviset' weist das EKW die Bedeutungsexplikation (3) zu:

(3) $X \underline{\text { zavisit }}$ ot $B=$ 'B možet kauzirovat' izmenenija $v$ svojstvach, sostojanijach ili povedenii $x$-a.' (APRESJAN 1974b, 108)

Die lexikographische Definition (3) stellt im Hinblick auf den Kontext, in dem das Stichwort expliziert wird, eine Konversion dar. Unsere Testergebnisse lassen vermuten, daB der Grund für die geringe Verstehbarkeit dieser Bedeutungsexplikation in der doppelten gedanklich-begrifflichen Operation zu suchen ist, die unsere Versuchspersonen vollziehen mußten (vgl. S. 195). Neben der Reduktion der unterstrichenen Bedeutungskomponenten auf ein Verb muBten sie die durch Wortformen der Lexeme načal'nik und brigadir realisierten Mitspieler der durch zaviset, bezeichneten Situation umstellen; vgl. das Satzpaar (4) - (5): 
(4) Načal'nik možet pričinjat' izmenenija $v$ svojstvach, sostojanijach ili povedenii brigadira. $\rightarrow$

(5) Brigadir zavisit ot načal'nika.

Es scheint daher angebracht, zur weiteren Steigerung seines lexikographischen Nutzwertes die Bedeutungsexplikationen des EKW nicht so zu konstruieren, daB die lexikographischen Definitionen bezüglich des Kontextes, in dem das Stichwort expliziert wird, eine Konversionsstruktur aufweisen. 58

Im Gegensatz zur lexikographischen Definition von zaviset' scheint die geringe Verstehbarkeit, die wir aufgrund unserer Testergebnisse der Bedeutungsexplikation von jasnyj zuweisen müssen, nicht auf strukturelle Besonderheiten der Explikation zurückzuführen sein. Die Tatsache, daß 5 Versuchspersonen bei der Reduktionsparaphrasierung der lexikographischen Definition von jasnyj (in der hier betrachteten Bedeutung) eine Wortform des Lexems pustoj verwendeten, deutet eher darauf hin, daß eine ganz bestimmte Bedeutungskomponente der Explikation nicht richtig verstanden wurde; vgl. die Reduktionsparaphrasierung von (6) in (7):

(6) Nebo-charakterizujuščesja otsutstviem $v$ atmosfere častic (naprimerl= oblaka, pyl', dym, tuman ...), prepjaststvujušcich vosprijatiju sveta.

(7) Nebo pustoe.

Augenscheinlich haben fünf der sechs Versuchspersonen die Bedeutungskomponenten 'otsutstviem častic' nicht dekodieren können, obwohl sie durch den in klammern stehenden Semkomplex illustriert bzw. extensional definiert werden (vgl. S. $109 \mathrm{f.}$.). Die schlechten Testergebnisse, die in unserem Experiment bei der lexikographischen Definition von jasnyj erzielt wurden, scheinen unsere Auffassung zu bestätigen, daB sich im EKW entgegen der lexikologischen Prinzipien seiner Konstrukteure Definitionen finden, die alles andere als das naive Weltbild des Durchschnittssprechers explizieren. Die Verwendung von Termini aus der Fachsprache von Physik und Meteorologie wie 'atmosfera, častica, vosprijatie sveta' 
in der oben genannten lexikographischen Definition, die genauso gut aus einem (populär)wissenschaftlichen Nachschlagewerk zur meteorologischen Terminologie stammen könnten, führt dazu, daB die Konstrukteure des EKW ihrer Intention untreu werden und einen (quasi)wissenschaftlichen Begriff von jasnyj explizieren ( $v g l$. hierzu auch S. $94 \mathrm{ff.}$ ).

Stellt man die Bedeutungsexplikation (6), die das EKW jasnyj zuweist, der OZEGOVschen Explikation (8) gegenüber, die in unserem Versuch von allen Informanten richtig dekodiert wurde, so scheint unsere Vermutung bestätigt:

(8) Nebo jasnoe = 'Nebo ničem ne sdelano tëmnym ili ničem tëmnym ne zakryto, ne propuskajuščim sveta.' (OZEGOV 1973, 842)

Die lexikographische Definition (8) verwendet weder fachsprachliche Bezeichnungen für Bedeutungskomponenten, noch expliziert sie einen (quasi)wissenschaftlichen Begriff von jasnyj.

Die Testergebnisse für die lexikographischen Definitionen des EKW sind nicht nur geeignet, korrektive Funktionen auf bestimmte Bedeutungsexplikationen einzelner Lexeme auszuuben, sie werfen auch ein interessantes Licht auf die Kompetenz-Performanz-Problematik in der Linguistik. Die lexikographischen Definitionen des EKW, die wir in unseren Versuch miteinbezogen haben, weisen unterschiedliche Längen auf. Geht man davon aus, daß die Länge einer Bedeutungsexplikation ungefähr dem Grad ihrer Verästelung oder syntaktischen Komplexität entspricht, die sich beispielsweise mit Hilfe einer Dependenzgrammatik ermitteln lieBe, so bieten die Ergebnisse unseres Experiments keinen Beleg dafür, daB ein hohes MaB an syntaktischer Komplexität sich auch erschwerend auf die Dekodierung der entsprechenden lexikographischen Definitionen auswirkt. Die guten Resultate, die die relativ zu den Explikationen von beispielsweise drobit', zaviset, und jasnyj syntaktisch viel komplexeren lexikographischen Definitionen von ostorožnyj und provocirovat' er$z i e l t e n, z e i g e n$ deutlich, daB zwischen systemlinguistisch 
spezifizierter Komplexität syntaktischer Strukturen und Performanzerscheinungen, zu denen u.a. die Dekodierung dieser Strukturen zu rechnen ist, keine Isomorphie angesetzt werden darf (HORMANN 1978, 51ff.; LEUNINGER 1974, 55ff.).

\subsection{Zusammenfassung}

Die Fähigkeit, mit der Definitionssprache des Wörterbuchs von OZEGOV zu konkurrieren, die der SemS des EKW aufgrund unserer Testergebnisse zuzumessen ist, wirft ein positives Licht auf den Anspruch, den die Konstrukteure des EKW mit ihrem Wörterbuch für die Verbesserung der lexikographischen Beschreibungspraxis in den gängigen russischen Definitionslexika erheben. Unser Experiment relativiert erheblich die Skepsis und die vielen, oft mit überzogener und polemischer Kritik vorgetragenen Zweifel, die von verschiedenen Autoren an der Tauglichkeit der Sems für lexikographische Bedirfnisse angemeldet worden sind. Die uns zur verfügung stehenden Testergebnisse erlauben es nicht, wie beispielsweise KOTELOVA behauptet, der SemS des EKW pauschal jegliche Eignung für die Erstellung von Definitionslexika abzusprechen, nur weil es sich um eine künstliche Definitionssprache handelt. Die starke Reglementiertheit, der die Ausdrucksmittel der SemS des EKW unterliegen, führt zwar dazu, daB in der SemS abgefaßte lexikographische Definitionen im Vergleich zu solchen, die sich einer natürlichen Definitionssprache bedienen, of stilistische und/oder grammatische Abweichungen aufweisen, die Resultate unseres Versuchs geben jedoch keinerlei Anhaltspunkte dafü, daB diese spezifischen Eigenschaften der SemS des EKW sich negativ auf ihre Effektivität für die Explikation von Wortbedeutungen auswirkt.

Die Ergebnisse unseres Experiments legen die Fortfiurung des zur Zeit leider gestoppten Vorhabens der Konstrukteure des MST nahe, zunächst ein einsprachiges EKW des Russischen und $z u$ einem späteren Zeitpunkt zweisprachige (z.B. russ.engl., russ.-franz. und russ.-dt.) EKW's zu erstellen. 59 
Unser Experiment läßt vermuten, daß eine Weiterführung des Projekts, dessen theoretischer Beitrag für die Semantik und Lexikologie wohl nie ernsthaft in $Z$ weifel gezogen werden kann, auch von praktischem Nutzwert für die Lexikographie sein wird. 


\section{AnMERKungen}

Im folgenden bezeichnen wir die drei genannten Forscher kurz als die Konstrukteure des MST. Ober die unterschiedlichen Anteile, die die drei Sprachwissenschaftler an der Ausarbeitung des Modells hatten, vgl. REUTHER 1978, 35. In der vorliegenden Arbeit haben wir mit Ausnahme von IORDANSKAJA/MEL'CUK 1980 und SCHMIDT 1981 nur einschlägige Veröffentlichungen der Konstrukteure des MST berücksichtigt, die bis zum Jahre 1975 erschienen sind. Spätere Publikationen, in denen zum Teil Modifikationen am MST vorgeschlagen werden, die jedoch die Grundkonzeption des erklärend-kombinatorischen wörterbuchs nicht verändern, haben wir in das Literaturverzeichnis aufgenommen, um bibliographische Vollständigkeit zu erzielen. Die wichtigsten Neuerungen am MST, die in der nach 1975 erschienenen Literatur gemacht werden, sind 1) Aufgabe der Kasusrollen in der formalen Notationsvariante der semantischen Sprache des erklärend-kombinatorischen wörterbuchs (APRESJAN 1980, 10, 98; MEL'CUK 1974C I, 41), 2) Untergliederung der Ebene der semantischen Repräsentation in die Ebenen einer Tiefen- und einer Oberflächensemantik (APRESJAN 1980, 10-14) und 3) Präzisierungen des Begriffs der Wohlgeformtheit von Texten u.a. im Zusammenhang mit den Selektionsrestriktionen des Rektionsmodells (APRESJAN 1978).

${ }^{2}$ vgl. hierzu auch HORMANN $1978,33-59$.

3 Im Zusammenhang mit der Darstellung des MST muB die Arbeit APRESJAN $1974 \mathrm{~b}$ kritisch erwähnt werden. Einerseits bespricht der Autor das Modell in seiner ersten Fassung (APRESJAN 1974b, 36-56), andererseits geht er in seinen Oberlegungen zur semantisch-lexikalischen Beschreibung im MST von der zweiten Fassung des Modells aus. Diese Mängel dürften nicht zuletzt auf die Tatsache zurückzuführen sein, daB es sich bei APRESJAN $1974 \mathrm{~b}$ u.a. Um eine schlecht zusammengestellte, partielle Kompilation bereits früher veröffentlichter Arbeiten handelt. 
${ }^{4}$ Eine Klassifikation der verschiedenen Faktoren, die die Konstrukteure des MST implizit für Textsynonymie verantwortlich machen, findet sich in SCHMIOT 1981.

${ }^{5}$ In der ersten Fassung des MST erfolgt die synonymische Paraphrasierung von einer lexikalisch-syntaktischen Basisstruktur aus (ZOLKOVSKIJ/MEL'CUK 1967, 11), in seiner zweiten Fassung von mehreren (MEL'ČK 1974b, 148-149).

${ }^{6}$ Beim EKW handelt es sich nicht um ein fertiges Wörterbuch. Die Konstrukteure des MST und ihre Mitarbeiter haben bisher über verschiedene Publikationen verstreut lediglich ein Fragment des Lexikons vorgelegt, das komplette Wörterbuchartikel und einzelne lexikographische Definitionen verschiedener russischer Lexeme umfaßt. Wir verwenden den Terminus EKW im folgenden, um uns sowohl auf dieses Wörterbuchfragment als auch auf die ihm zugrundeliegenden lexikologischen Prinzipien zu beziehen.

7 In zwei ihrer Veröffentlichungen erheben die Konstrukteure des MST auch den Anspruch, das EKW für Zwecke der Sprachvermittlung einsetzen zu können (APRESJAN/ZOLKOVSKIJ/MEL'CUK 1969d; MEL'CUK 1974b, 107).

${ }^{8} \mathrm{Vgl}$. in diesem Zusammenhang allerdings auch APRESJAN 1968 , 35. Dort äuBert der Autor die Vermutung, daB die erforderliche Anzahl der "Wörter" der SemS des EKW ungefähr dreihundert umfassen dürfte.

9 In partieller Anlehnung an die Praxis der Konstrukteure des MST bedienen wir uns der folgenden Notation: Objektsprachliche Einheiten, zu explizierende objektsprachliche Einheiten und Einheiten der SemS werden kursiv gedruckt. Zu explizierende objektsprachliche Einheiten werden zusätzlich unterstrichen, Z.B. razbivat' $=$ '..', Einheiten der SemS werden zusätzlich in ' gesetzt z.B. 'kauzirovat', 'cel'', ' $A$ '. AuBerdem werden alle transliterierten russischen Aus- 
drücke (mit Ausnahme von Literaturangaben und Autorennamen) kursiv gedruckt.

10 Das Prinzip des erweiterten Lexikons findet auch in gängigen Definitionswörterbüchern Verwendung und wird dort wie beispielsweise im Definitionswörterbuch von OZZEGOV (OZEGOV 1973, 11-12) als lexikologisches Prinzip der Kürze der Explikation (kratkost' tolkovanija) bezeichnet (vgl.S. 152 f.)

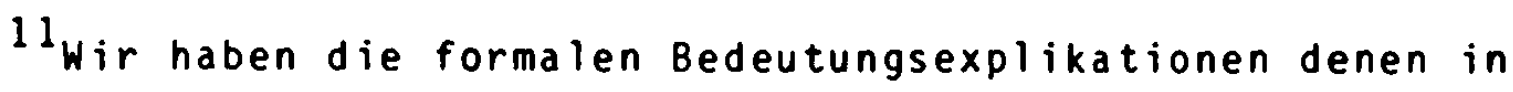
APRESJAN $1974 b$ angeglichen, indem wir die Valenzen der Prädikate numeriert und die Kopula ' $i$ ' als eine koordinative Dependenzrelation dargestellt haben (APRESJAN $1974 b, 78,205)$.

${ }^{12}$ Sollte sich REUTHERs Hinweis auf das Kunstwort 'kauzirovat' ' beziehen, das als einzige Einheit in die Sems eingeht, die in Anlehnung an das Lateinische gebildet ist, jedoch gelegentlich auch durch 'pričinjat' ' bezeichnet wird, so verallgemeinert er auf jeden Fall zu stark.

$13 \mathrm{Vgl}$. S. $146 \mathrm{ff}$. zum Versuch, den potentiellen Benutzerkreis des EKW zu bestimmen.

14 Es ist unverständlich, daB nur in APRESJAN 1969 c bestimmte Ausdrücke der Sems auf diese Weise spezifiziert werden.

${ }^{15}$ Dies gilt zumindest für die wortsprachliche (= lexikographische) Variante der Sems. Eine Spezifizierung der Bedeutung von 'kauzirovat' als 'delat' tak, čtoby' findet sich - allerdings nicht im Hinblick auf dessen Verwendung in der Sems der EKW - bei MEL'CUK (MEL'CUK 1974b, 67).

${ }^{16}$ Da die Grenze zwischen lexikalischer Homonymie und lexikalischer Polysemie fließend ist und nur willkürlich gezogen werden kann, entscheiden wir uns für den Terminus 
"Homonymie". Mit ihm bezeichnen wir all diejenigen Fälle, in denen einer Bedeutungskomponente der SemS mehrere, wie auch immer zueinander in Beziehung stehende Inhalte zugewiesen werden können. M.a.W., die Unterscheidung zwischen (lexikalischer) Homonymie und (lexikalischer) Polysemie bildet für uns keine Dichotomie.

${ }^{17}$ Die kanonische Form der lexikographischen Definition des EKW besteht darin, daB sowohl im Definiens als auch im Definiendum Gegenstandsvariable vermerkt sind, die entsprechend den Regeln der russischen Grammatik verändert werden. Zahlreiche Definitionen im EKW sind jedoch hinsichtlich der Behandlung der Gegenstandsvariablen ziemlich nachlässig formuliert worden: in Bedeutungsexplikationen von Adjektiven und Substantiven finden sich gelegentlich überhaupt keine Bezeichnungen für Gegenstandsvariable, manchmal werden die entsprechenden Bezeichnungen vertauscht, oft werden sie nur teilweise oder gar nicht entsprechend den Kongruenzregeln der russischen Grammatik verändert. Wir werden im folgenden, sofern es erforderlich ist, die von uns zitierten lexikographischen Definitionen des EKW an ihre kanonische Form anpassen.

18 In Anlehnung an FILLMOREs Definition des Tiefenkasus 'Agentive' (FILLMORE 1968, 24) verstehen wir die Agensrolle als eine semantische Relation, in die ausschlieblich belebte, d.h. menschliche Mitspieler als Anstifter einer Handlung zu anderen Mitspielern treten können. In unseren Oberlegungen zu den kausativen Verben entspricht der Agensrolle, die ein Mitspieler einnehmen kann, daher stets die einer menschlichen Kausalinstanz. Unser Verständnis der Agensrolle unterscheidet sich somit vom Begriff der Subjektrolle APRESJANS (APRESJAN 1974b, 127).

${ }^{19}$ Die eindeutig nichtintentionale Lesart von (23') kann mit gleichem Recht auch aus dem Wissen hergeleitet werden, daB es sich um einen gestiefelten und gespornten Husaren han- 
delt, der sich unsicher (naošǒup') zu seinem Ziel bewegt.

${ }^{20}$ Ein lexikalisches Aquivalent für das intentionale Verb des Deutschen ermorden existiert im Russischen nicht, da ubivat, sowohl eine intentionale als auch eine nichtintentionale Tötung bezeichnen kann.

21In den Bedeutungsexplikationen des EKW werden Gerundialkonstruktionen - augenscheinlich unterschiedslos - in antepositionaler (vgl. ubezdat' S. 207) und postpositionaler Stellung (vgl. upuskat' iz vidu) verwendet.

${ }^{22}$ Da es sich bei der Merkmalsanalyse um ein mengentheoretisches Modell handelt, sind die semantischen Merkmale in dessen Beschreibungen beliebig kommutierbar.

${ }^{23}$ Aus rein linguistischer sicht, in der das EKW als "... ein grundlegendes theoretisches Werk zur linguistischen Semantik, das den gleichen wissenschaftlichen Status wie die theoretische Grammatik hat ..." (APRESJAN 1969a, 11) angesehen werden kann, besteht selbstverstandlich kein AnlaB $z u$ bezweifeln, ob es sinnvoll ist, Bedeutungsexplikationen in das Lexikon aufzunehmen.

24 Ist es einerseits problematisch, ein potentielles Bedurf$n$ is an Wörterbuchdefinitionen bei Sprechern ins Feld $z u$ füren, um die Erstellung eines nichtfachsprachlichen Definitionslexikons zu rechtfertigen, so lassen sich andererseits entsprechende lexikographische Bemühungen immer noch durch das - von den Konstrukteuren des MST allerdings nicht erwähnte - Interesse begrüden, die lexikalischen Bedeutungen der Wörter einer Sprache zu katalogisieren und, falls beabsichtigt, zu normieren.

${ }^{25}$ Leider versäumt es MEL'CuK, zu sagen, ob er das verb zabyvat, als polysem betrachtet oder nur eine seiner Bedeutungen beschreibt. Die Verwendung von zabyvat", wie sie beispielsweise in den Wortverbindungen zabyvat' 
buterbrody und zabyvat' zakryt' dver' vorliegt, erfaßt die oben genannte Bedeutungsexplikation nicht.

${ }^{26}$ Nach einer persönlichen Mitteilung von REUTHER soll der Semkomplex 's cel' $j u$ ispravit' $3-a$ ' notwendig sein, um nakazyvat, von mstit' semantisch unterscheiden zu können. Dieser Einwand scheint uns nicht haltbar, da es für die Bedeutung von mstit, paradigmatisch ist, daB der Mitspieler in der Objektrolle nicht ein Vergehen mit beliebigen Folgen begangen hat, sondern genau ein solches, dessen Auswirkungen den Mitspieler in der Agensrolle treffen.

${ }^{27}$ Ein Beispiel dafür, daß die Unbestimmtheit der Bedeutungen sprachlicher Zeichen auch für solche Lebensbereiche Folge haben kann, in denen Menschen mit Hilfe sprachlicher Regelungen bestimmte Verhaltensweisen verabreden bzw. rechtlich verbindlich festlegen, schildert DUBISCHAR anhand des sogenannten Fensterfalls (DUBISCHAR 1974, 87ff.).

${ }^{28}$ REUTHER zufolge gibt die lexikographische Definition von kofe übrigens auch das "naive" Weltbild des deutschen Durchschnittssprechers wieder, da er sie in seinem ersten Versuch zu einem russisch-deutschen EKW einfach übersetzt und dem deutschen Wort Kaffee zuweist.

${ }^{29}$ Im Gegensatz zum Lexem osël 1 , das nur in der Bedeutung 1b. ('Samec osza la.') iuber eine lexikalische Konnotation verfügt, halten es IORDANSKAJA/MEL'CUK für evident (estestvennym), daB im Russischen die hier genannte Konnotation dem hinsichtlich Geschlechtszugehörigkeit unspezifizierten Lexem ǐsak 1a. zukommt (IORDANSKAJA/MEL'CUK $1980,196)$.

${ }^{30}$ IORDANSKAJA/MEL'CUK räumen ein, daB der oben genannte definitorische Zusammenhang zwischen beiden Begriffen nicht für alle Erscheinungen, die (im Russischen) unter den Begriff der lexikalischen Konnotation subsumiert werden, gilt: "... wir kennen einen Fall, in dem das Vorhan- 
densein einer bestimmten Konnotation bei einem Lexem L mehr oder weniger evident ist, aber dennoch ein Lexem L' (genauer: eine lexikalische Einheit $L^{\prime}$ ) in der gegebenen Sprache nicht existiert. Das Lexem tëšča = 'Mat' ženy' hat zwei Konnotationen: 1. 'Predupreditel'naja zabot $i$ vost' ', 2. 'Razdražajuščaja pomecha v ličnoj žizni'. Der Ausdruck mat' ženy, der mit dem Wort tëšča absolut synonym ist, hat diese Konnotation nicht. Dabei handelt es sich bei dem Lexem tëšca weder um ein Lexem, das zu diesem in Polysemierelation steht und die Bedeutung 'razdražajuščaja pomecha' hat noch um ein abgeleitetes Lexem mit einer annähernd gleichen Bedeutung ... Da der Status von Konnotationen dieses Typs uns unklar ist, haben wir es uns erlaubt, von ihnen in unserer Darlegung ... völlig zu abstrahieren." (IORDANSKAJA/MEL'CUK 1980, $208-209)$

${ }^{31}$ Ein erster Versuch, die lexikalischen Funktionen des EKW zur Konstruktion von Wortschatzübungen im Russischunterricht für englische Muttersprachler einzusetzen, findet sich in LEED/NAHKIMOSKY 1979.

${ }^{32}$ MEL'CUK deutet an gleicher stelle an, daB es auch möglich sei, lexikalische Funktionen bei zwei und mehr Argumenten ( = Syntagmen) zuzulassen, so daß der einschränkende Klammerzusatz entfallen kann; vgl. die beiden folgenden Notierungen der lexikalischen Funktion Magn bei krasnyj (ot moroza) und krasnyj (ot styda):

Magn (krasnyj, ruki, ot moroza) = kak gusinye lapy Magn (krasnyj, $l_{i c o}$, ot styda) = kak varenyj rak (MEL'CUK $1974 b$, ebd.)

33 In KOTELOVA 1975 werden darüber hinaus noch das Bedeutungskonzept und die Beschreibung der Kombinierbarkeit von Lexemen im EKW behandelt. Neben der Kritik am EKW finden sich innerhalb der sowjetischen Linguistik noch kritische Stellungnahmen zum MST als einer Sprachtheorie oder Gram- 
matikmodell. Insbesondere sind hier die Arbeiten von ZVEGINCEV (ZVEGINCEV 1973, 163ff.; 1976, 205ff.) zu nennen.

${ }^{34}$ Wir betrachten unser Experiment als eine Vorausanalyse, die mögliche Wege für die zukünftige forschung in der linguistischen Semantik aufzeigen soll. U.a. aufgrund der Signifikanzproblematik kann unser Versuch nur den Status einer Vorausanalyse beanspruchen, weil wir nur einen Ausschnitt der SemS des EKW in das Experiment miteinbeziehen. AuBerdem haben wir den Test nicht in einem bestimmten Zeitraum unter gleichen Versuchsbedingungen wiederholt. Wir können daher nicht mit Sicherheit behaupten, daß die Streuung der Testergebnisse nicht zufallsbestimmt und das einmalige, von uns ermittelte Testverhalten der Informanten generalisierbar ist. $\mathrm{Vgl}$. S. $147 \mathrm{f} ., 167 \mathrm{f}$. für weitere Umstände, die potentielle Störfaktoren für die Aussagekraft unseres Experiments darstellen.

${ }^{35_{M i t}}$ dem Terminus "Reduktionsparaphrasierung" bezeichnen wir die Operation der Umformung, ihr Resultat mit dem Terminus "Reduktionsparaphrase".

${ }^{36} \mathrm{Vgl}$. Zur Testanweisung $\mathrm{S} .221 \mathrm{ff}$. Theoretisch erwartbar und in diesem Kontext mit udaljat'sja äquivalent ist auch eine Wortform des Verbs otplyvat'.

${ }^{37}$ Um die lexikographischen Definitionen des Wörterbuchs von OZEGOV, in denen Verben in der Regel im perfektiven Aspekt expliziert werden, den Bedeutungsexplikationen des EKW, die Verben stets im imperfektiven Aspekt definieren, anzugleichen, formen wir im folgenden die OZEGOVschen Bedeutungsexplikationen bezüglich der Aspektzugehörigkeit der in ihnen verwendeten Verben um. Eine derartige Veränderung unseres Testmaterials ist immer dann zulässig, wenn die Gesamtbedeutung der Definition nicht affiziert wird; $v g l$. in diesem Zusammenhang die Aspektveränderung der Ex- 
plikation von usvoit' S. 211. Unser Vorgehen entspricht dem der Konstrukteure des MST in ihren kritischen Analysen der lexikographischen Definitionen gängiger russischer Wörterbücher (APRESJAN 1969a, 13, Anm. 6).

${ }^{38}$ Unsere Oberlegungen werden im übrigen durch die Befragung eines russischen Informanten zu der potentiellen Ambiguität von 'proizvodit' tolcok' im Hinblick auf die Implikation bzw. Nichtimplikation von 'prikasat'sja' bzw. 'privodit' kratkovremenno $v$ kontakt gestützt.

${ }^{39}$ Die partielle Inkonsequenz, mit der die Konstrukteure des MST dieses Postulat in der lexikographischen Beschreibungspraxis einlösen (vgl. S. 46 ff.), beeinträchtigt unsere Prämisse nicht.

${ }^{40}$ Die unter (21) angeführte Definition findet sich in $z$ irkulärer Form auch im BAS; vgl. Lovkij = 'Obladajuščij fizičeskoj snorovkoj' (BAS 1969, Bd. 6, 311) und snorovka = 'Lovkost'' (BAS 1969, Bd. 13, 1506).

4l In der APRESJANschen Terminologie ist die Bedeutung des Lexems čuvitvo als rodovoe značenie, die des Lexems priznatel'nost' als vidovoe značenie zu bezeichnen (APRESJAN 1974b, 89).

42 Zirkularität weisen auch die Explikationen im BAS auf; vgl. priznatel'nost' $=$ 'Cuvstvo blagodarnosti za uslugu' (BAS 1969, Bd. 11, 487) und blagodarnost' = 'Cuvstvo priznatel'nosti' (BAS 1969, Bd. 1, 477).

${ }^{43}$ Aus diesem Grund entfalten wir die lexikographische Definition, die OZEGOV udarjat' zuweist, in $\underline{\text { udarjat' }}=$ 'Proizvodja rezkij tolčok, prikasat'sja s siloj $k$ komu/čemu-n.' (vgl. S. 207 f.).

Die geringe Reglementiertheit der Definitionssprache des wörterbuchs von OZ̈EGOV, die, wie oben gezeigt, zu redun- 
danten lexikographischen Definitionen führen kann, steht naturlich im Widerspruch zu OŽEGOVs lexikologischem Prinzip der Kürze der Bedeutungsexplikation (vgl. S. 152 f.).

${ }^{44}$ Bei unserer Vermutung, daß Semredundanzen einen potentiellen Störfaktor für unser Experiment darstellen, handelt es sich selbstverständlich um eine Annahme, die wir im Hinblick darauf, wie durch sie die Validität unseres Tests beeinflußt wird, nicht ubberpruft haben.

${ }^{45}$ Die Argumente, die sich zur Beschreibung der Kombinierbarkeit von Lexemen in APRESJAN 1978 finden, präzisieren 2 war die Behandlung von Sinnanomalien im MST bZW. im EKW, sind aber für die hier in Rede stehende Problematik besonders im Hinblick auf die bei APRESJAN $1974 \mathrm{~b}$ und MEL'CUK $1974 \mathrm{~b}$ geubte Beschreibungspraxis nicht erhellend.

45 Im folgenden greifen wir für die Bezeichnung der Kasusrollen, die in den lexikographischen Definitionen des EKW und des Wörterbuchs von OZEGOV angesetzt werden, auf das Inventar an Kasusrollen zurück, das APRESJAN zur semantischen Beschreibung in APRESJAN $1974 \mathrm{~b}, 125 \mathrm{ff}$. benutzt.

${ }^{47}$ Dem in (1) eliminierten Semkomplex entspricht im EKW ein spezieller Wörterbuchartikel; vgl. arestovyvat' 2:

$X$ arestovyvaet $W Y-a=$ 'Oficial'nye organy $X$ lis̆ajut $Y-a$ vozmožnosti rasporjažat'eja $W$ kak sobstvennost'ju.'

Vlasti arestovali ves' tiraž večernej gazety. (MEL'CUK 1974b, 117)

Ein weiterer Unterschied zum Wörterbuch von OZ̈EGOV besteht darin, daB MEL'CUK die Verwendung von arestovyvat' in dieser Bedeutung als archaisch (ustarezoe) bezeichnet (MEL'CUK $1974 b$, ebd.).

${ }^{48}$ Im Falle der Bedeutungsexplikationen von prenebregat, (EKW) und seinem Antonym učityvat' (EKW) dürfte das Auftreten einer homogenen Hemmung ausgeschlossen sein, da die Komponente 
'ne-' in 'nesuščestvenen' gegenüber 'suščestvenen' (vgl. S. 203, 210) kraft ihrer Bedeutung beide Bedeutungsexplikationen in einen starken Kontrast zueinander stellt.

${ }^{49}$ Es handelt sich bei den Verben 'nanosit' ' und 'proizvodit'" um verschiedene phraseologisch gebundene Ausdrucksmittel bezijglich udar, die je nach Besetzung der Kasusrolle 'Obj' durch Wortformen von belebten bzw. unbelebten Substantiven ausgewählt werden muissen.

${ }^{50}$ Die Schwierigkeiten, auf die wir gestoßen sind, um allein die oben genannte Anzahl an Versuchspersonen zusammenzubekommen, waren beträchtlich, zumal uns zunächst angebotene Hilfe durch die diplomatische Vertretung der Sowjetunion in der Bundesrepublik unerwartet und keineswegs einsichtig begriundet verweigert wurde.

${ }^{51}$ Bei sieben Versuchspersonen handelte es sich um deutschstämmige Aussiedler aus der Sowjetunion, die zum Teil länger als drei Jahre in der Bundesrepublik wohnten. Trotz der potentiellen Beeinträchtigung, die dieser Umstand für ihre russische Sprachbeherrschung und zwangsläufig auch für die Homogenität der Gruppe unserer Versuchspersonen darstellt, haben wir diese Informanten zu unserem Experiment zugelassen.

${ }^{52}$ Diese zusätzliche Erläuterung erhielt nur die Informantengruppe, der wir die lexikographischen Definitionen des EKW vorgelegt haben. Da wir trotz aller Bemijhungen, Fehler zu vermeiden, bei der Aufarbeitung des Testmaterials aus dem Wörterbuch von OZEGOV nicht mit letzter Sicherheit ausschließen konnten, stilistische bzw. eventuell sogar grammatische Abweichungen zugelassen zu haben, gaben wir - sozusagen vorbeugend - den Versuchspersonen, die diese lexikographischen Definitionen zu paraphrasieren hatten, einen entsprechenden mindlichen $H$ inweis. 
${ }^{53}$ Bei dem Informanten handelte es sich um einen sowjetischen Lektor, der ïber keine Deutschkenntnisse verfügte.

${ }^{54}$ Wenn wir in diesem Zusammenhang von Bedeutungsunterschieden sprechen, so machen wir uns die begriffskritischen Oberlegungen in APRESJAN 1974a und 1974b, 243-248 zueigen und setzen uns bewuBt von der Terminologie in EVGEN'EVA 1970 , 17 und 1975, 8 ab, wo der Terminus "Bedeutungsnuance" (smyslovoj ottenok) verwendet wird (s.u.).

${ }^{55} \mathrm{Vgl}$. hierzu auch APRESJAN 1971a, 32; CHOMSKY 1973, $102-$ 107 ; WELTE 1974, 198-202.

${ }^{56}$ Bei dieser Verpflichtung handelt es sich wohl gemerkt nicht um eine (Sprechhandlungs) Obligation im Sinne WUNDERLICHS (WUNDERLICH 1972, $20 \mathrm{ff.}$ ).

${ }^{57}$ Von den vier verschiedenen Bedeutungsexplikationen, die OZEGOV predZagat' zuweist, sind nur drei Explikationen im eigentlichen Sinne, da unter 2. lediglich Angaben zur Kombinierbarkeit der Stichworts mit anderen Lexemen gemacht werden, die mit Hilfe einer lexikalischen Funktion beschrieben werden können; $v g l$. Oper 1 (vopros) = zadvat'.

${ }^{58}$ Eventuell muB unsere SchluBfolgerung durch einen potentiellen Störfaktor relativiert werden, der in der lexikalischen Auffïllung der Kasusrollen von zaviset' durch Wortformen der Lexeme načal'nik und brigadir gesehen werden kann. Innerhalb einer Abhängigkeitshierarchie bezeichnen beide Lexeme verhältnismäßig hohe Positionen, die nur wenig voneinander entfernt sind. Unter diesem Aspekt hätte die Ersetzung eines der Wörter durch beispielsweise eine Wortform des Lexems rabočij eventuell zu besseren Testergebnissen geführt.

${ }^{59}$ Ansätze für diese drei Wörterbuchtypen finden sich in APRESJAN/MEL'CUK/ZOLKOVSKIJ 1973C/ZOLKOVSKIJ/MEL'CUK 1971 und REUTHER 1978. 
10. LITERATURVERZEICHNIS

Alperin, V.A./Apresjan, Ju.D./Mel'čuk, I.A.

1975 "Materialy $k$ tolkovo-kombinatornomu slovarju russkogo jazyka: 3 slovarnye stat'i", Predvaritel'nye publikacii problemnoj gruppy po éksperimental'noj $i$ prikladnoj lingristike Instituta Russkogo Jazyka AN SSSR, vyp. 62 (Moskva), 18-24

Apresjan, Ju.D.

1963 "Sovremennye metody izučenija značenij $i$ nekotorye problemy strukturnoj lingvistiki", in: Problemy strukturnoj lingvistiki (Moskva), 102-150

1966 Idei $i$ metody sovremennoj strukturnoj lingvistiki (Moskva)

1967 Éksperimental'noe issledovanie semantiki russkogo glagola (Moskva)

1968 "Ob éksperimental'nom slovare russkogo jazyka", Voprosy jazykoznanija 5, 34-39

1969a "Tolkovanie leksičeskich značenij kak problema teoretičeskoj semantiki", Izvestija AN SSSR, Serija lit. i jazyka $28, \mathrm{Nr} .1,11-23$

Apresjan, Ju.D./Zolkovskij, A.K./Mel'čuk, I.A.

1969b "Semantics and Lexicography: Towards a New Type of Unilingual Dictionary", in: Kiefer, F. (ed.) Studies in Syntax and Semantics (Dodrecht), 1-33

Apresjan, Ju.D.

1969 c "O jazyke dlja tolkovanija značenij slov", Izvestija AN SSSR, Serija lit. i jazyka 28, Nr. 5, 415-428

Apresjan, Ju.D./Zolkovskij, A.K./Mel'čuk, I.A.

$1969 d$ "Ob odnom sposobe izučenija sočetaemosti slov", Russkij jazyk $v$ nacional'noj škole $6,61-72$ 
Apresjan, Ju.D.

$1969 e$ "Sinonimija i sinonimy", Voprosy jazykoznanija 4, $75-91$

1971 a "O nekotorych diskussionnych voprosach teorii semantiki", Voprosy jazykoznanija 1, 23-36

$1971 b$ "O reguljarnoj mnogoznačnosti", Izvestija AN SSSR, Serija lit. i jazyka 30, Nr. 6, 509-523

Apresjan, Ju.D./Mel'čuk, I.A./Salapina, Z.M.

1973a "Materialy $k$ tolkovo-kombinatornomu slovarju russkogo jazyka: 2 slovarnye stat'i", Predvaritel'nye publikacij problemnoj gruppy po éksperimental'noj i prikladnoj lingvistike Instituta Russkogo Jazyka AN SSSR, vyp. 38 (Moskva), 3-18

Apresjan, Ju.D./Mel'čuk, I.A./Percov, N.P.

$1973 b$ "Materialy k tolkovo-kombinatornomu slovarju russkogo jazyka: E slovarnych statej", Predvaritel'nye publikacii problemnoj gruppy po éksperimental'noj i prikladnoj lingvistike Instituta Russkoga Jazyka AN SSSR, vyp. 38 (Moskva), 19-43

Apresjan, Ju.D./Mel'čuk, I.A./Zolkovskij, A.K.

1973c "Materials for an Explanatory and Combinatory Dictionary of Modern Russian", in: Kiefer, F. (ed.) Trends in Soviet Theoretical Linguistics (Dodrecht), $411-438$

Apresjan, Ju.D.

$1974 a$ "Značenie slova i ottenok značenija", Izvestija AN SSSR, Serija lit. i jazyka 33, Nr. 4, 320-330

$1974 b$ Leksičeskaja semantika - Sinonimičeskie sredstra jazyka (Moskva) 
Apresjan, Ju.D./Zolkovskij, A.K./Mel'čuk, I.A.

1975 "Materialy $k$ tolkovo-kombinatornomu slovarju russkogo jazyka: 10 slovarnych statej", Predvaritel'nye publikacii problemnoj gruppy po éksperimental'noj i prikladnoj lingvistike Instituta Russkogo Jazyka AN SSSR, vyp. 62 (Moskva), 25-47

1978 "Jazykovaja anomalija i logičeskoe protivorečie", in: Mayenowa, M.R. (ed.) Tekst. Jęzrk. Poetrka. (Wroclaw), 129-151

1980 Tipy informacii dlja poverchnostno-semantičeskogo komponenta modeli smysl $\rightarrow$ tekst (Wien), Wiener Slawistischer Almanach, Sonderband 1

Arbatskij, D.I.

1974 "O suščnosti tavtologi $v$ semantičeskich opredelenijach", Voprosy jazykoznanija 2, 56-63

BAS

1965 Slovar' sovremennogo russkogo literaturnogo jazyka $\checkmark 17$ tomach (Bol'šoj akademičeskij slovar'), 19481965 (Moskva).

Biedermann, J. (ed.)

1976 I.A. Mel'čuk - Das Wort zwischen Inhalt und Ausdruck (München)

Bierwisch, M.

1970 "On Classifying Semantic Features", in: Bierwisch, M./Heidolph, K.E. (eds.) Progress in Linguistics (The Hague), 27-50

Bogdanova, E.Ju./Nikitina, S.E./Mel'čuk, I.A.

1975 "Materialy $k$ tolkovo-kombinatornomu slovarju russkogo jazyka: 3 slovarnye stat'i", Predvaritel'nye publikacii problemnoj gruppy po éksperimental'noj i prikladnoj lingvistike Instituta Russkogo Jazyka AN SSSR, vyp. 62 (Moskva), 3-17 
Brekle, H.E.

1972 Semantik (München)

Chafe, W.L.

1971 "Directionality and Paraphrase", Language 47, 1-26

Chomsky, N.

1971 "Deep Structure, Surface Structure and Semantic Interpretation", in: Steinberg, 0.D./Jakobovits, A. (eds.) Semantics: An Interdisciplinary Reader in Philosophy, Linguistics and Psychology (Cambridge), $183-216$

1973 Aspekte der Syntaxtheorie (Frankfurt)

Dubischar, R.

1974 Vorstudium zur Rechtswissenschaft (Stuttgart)

Evgen'eva, A.P.

1970 Slovar' sinonimov russkogo jazyka $\vee 2$ tomach (Leningrad)

1975 Slovar' sinonimov (Leningrad)

Faust, M.

1978 "Wortfeldstruktur und Wortverwendung", Wirkendes Wort 6, 365-401

Fillmore, Ch.

1968 "The Case for Case", in: Bach, E./Harms, R.T. (eds.) Universals in Linguistic Theory (New York), $1-88$

1969 "Types of Lexical Information", in: Kiefer, F. (ed.) Studies in Syntax and Semantics (Oodrecht), 109-137

Funk-kolleg Sprache

1973 Funk-Kolleg Sprache Bd. II (Frankfurt) 
Gak, V.G.

1972 "K probleme semantičeskoj sintagmatiki", in:

Saumjan, S.K. (ed.) Problemy strukturnoj

lingvistiki 1971 (Moskva), 367-395

Helbig, G.

1974 Geschichte der neueren Sprachwissenschaft (Hamburg)

Helbig, G./Schenkel, W.

1973 Wörterbuch zur Valenz und Distribution deutscher Verben (Leipzig ${ }^{3}$ )

Hörmann, H.

1978 Meinen und Verstehen - Grundzüge einer psychologischen Semantik (Frankfurt)

Immler, $M$.

1974 Generative Syntax - Generative Semantik (München)

Iordanskaja, L.N.

1970 "Popytka leksikografičeskogo tolkovanija gruppy

russkich slov so značeniem čuvstra", Mă̌inny

Perevod i Prikladnaja Lingvistika 13, 3-26

Iordanskaja, L.N./Mel'čuk, I.A.

1980 "Konnotacija v lingvističeskoj semantike", Wiener Slawistischer Almanach 6, 191-210

Isačenko, A.V.

1972 "Figurative meaning, derivation and semantic features", in: Worth, D. (ed.) The Slavic Word (The Hague), 76-91

Juhász, J.

1970 Probleme der Interferenz (München) 
Karaulov, Ju.N.

1976 Obščaja i russkaja ideografija (Moskva)

Katz, J.J.

1971 Philosophie der Sprache (Frankfurt)

Kiselevskij, L.I.

1973 "O semantičeskich metajazykach tolkovych slovarej

i jazykach opisanija énciklopedij", in: Romanogermanskaja i slavjanskaja leksikografija - Sbornik teoretičeskich statej (Minsk), 62-89

1977 Jazyki i metajazyki enciklopedij $i$ tolkovych slovarej (Minsk)

Kopylenko, M.M.

1973 Sočetaemost' leksem v russkom jazyke (Moskva)

Kossek, N.B.

1973 "Materialy k tolkovo-kombinatornomu slovarju russkogo jazyka: 5 slovarnych statej", Predvaritel'nye publikacii problemnoj gruppy po éksperimental'noj i prikladnoj lingvistike Instituta Russkogo Jazyka AN SSSR, vyp. 37 (Moskva), 38-46

Kotelova, N.Z.

1974 "Iskusstvennyj semantičeskij jazyk (teoretičeskie predposylki)", Voprosy jazykoznanija 5, 48-63

1975 Značenie slova i ego sočetaemost' (Leningrad)

1977 "Leksičeskaja sočetaemost" slova v slovare", in: Sovremennaja russkaja leksikografija 1976 (Leningrad), $36-53$

Krysin, L.

1978 "Sovremennaja rusistika: leksikologija $i$ leksičeskaja semantika - Obzor rabot za 1970 1973 99.", Wiener Slavistischer Almanach 2, $183-208$ 
1980 "Sovremennaja rusistika: leksikologija i

leksičeskaja semantika - Obzor rabot za 1974 -

1977 gg." Wiener Slavistischer Almanach 5,

$243-263$

Kutschera, F.V.

1971 Sprachphilosophie (Minchen')

Leed, R.L./Nakhimovsky, A.0.

1979 "Lexical Functions and Language Learning", Slavic and East European Journal 23, 104-113

Leuninger, H. (et al.)

1972 Psycholinguistik - Ein Forschungsbericht (Frankfurt)

Lorenz, W./Wojtak, G.

1977 Zum Verhältnis von Abbild und Bedeutung (Berlin)

Lyons, J.

1972 Einführung in die Linguistik (München²)

1977 Semantics Bd. I (Cambridge)

Mel'čuk, I.A.

1961 "O nekotorych tipach jazykovych značenij", in:

Achmanova, 0.S. et al. (eds.) 0 točnych metodach issledovanija jazyka (Moskva), 33-39

1966 "K voprosu o vešnich različitel'nych èlementach: semantičeskie parametry i opisanie leksičeskoj sočetaemosti", in: To Honor Roman Jakobson, Essays on the Occasion of his Seventieth Birthday 11 October 1966, Vol. II (The Hague-Paris), $1340-1361$

1970 "K principam opisanija označaemych (o lingvističeskoj semantike)", in: Jazyk i čelovek - Sbornik 
statej v pamjati P.S. Kuzncevora (Moskva), $201-217$

1973 "Towards a Linguistic 'Meaning $\leftrightarrow$ Text'-Model", in: Kiefer, F. (ed.) Trends in Soviet Linguistics (Dodrecht), 33-57

1974 a "Ob odnoj lingvističeskoj modeli tipa'Smysl $\leftrightarrow$ Tekst', Izvestija AN SSSR, Serija lit. i jazyka 33, $\mathrm{Nr} .5,436-447$

1974b Opyt teorii lingvističeskich modelej'Smysl $\leftrightarrow$ Tekst' (Moskva)

$1974 \mathrm{C}$ "Ob odnoj modeli ponimanija tekstor na estestrenI/II nom jazyke (semantičeskaja teorija R. Senka)", Naučno-techničeskaja informacija, Serija 2, Nr. 6, $35-46, N r .8,33-44$

$1974 d$ "Grammatical meanings in interlinguas for automatic translation and the concept of grammatical meaning", in: Kiefer, F. (ed.) Machine Translation and Applied Linguistics Bd. 1 (Frankfurt), 95-113

Ožegov, S.I.

1973 Slovar' russkogo jazyka (Moskva10)

Padučeva, E.V.

1974 O semantike sintaksisa (Moskva)

Reuther, $T$.

1978 "Plädoyer für das Wörterbuch", Linguistische Berichte $27,25-48$

Schmidt, P.

1981 "Lexical Semantics in the 'Meaning $\leftrightarrow$ Text' Approach", Russian Linguistics (im Druck)

Schwarze, Ch.

1975 Einführung in die Sprachwissenschaft (Kronberg ${ }^{2}$ ) 
Sredova, N.Ju.

1970 "Nekotorye zamečanija po povodu stat'i Ju. D. Apresjana 'Sinonimija i sinonimy'", Voprosy jazykoznanija 3, 36-44

Ubin, N.I.

1969 "Vyraženie parametra Magn v russkom jazyke", Mašinny perevod i prikladnaja lingvistika 11 , 60-65

Učebnyj slovar' sočetaemosti

1978 Učebnyj slovar' sočetaemosti slov russkogo jazyka (Moskva)

Uluchanov, I.S.

1977 Slovoobrazovatel'naja semantika $v$ russkom jazyke (Moskva)

Vol'f, E.M./Iordanskaja, L.N./Telija, V.N.

1973 "Materialy $k$ tolkovo-kombinatornomu slovarju russkogo jazyka: 5 slovarnych statej", Predvaritel'nye publikacii problemnoj gruppy po éksperimental'noj i prikladnoj lingvistike Instituta Russkogo Jazyka AN SSSR, vyp. 37 (Moskva), 3-23

Weinreich, $U$.

1967 "Lexicographic Definitions in Descriptive Semantics", in: Householder, F./Saporta, S. (eds.) Problems in Lexicography (Bloomington) 25-43

Welte, $H$.

1974 moderne linguistik: terminologie/bibliographie Bd. 1 (München) 
Wierzbicka, A.

1972 Semantic Primitives (Frankfurt)

1975 "Why 'Kill' Does not Mean 'Cause to Die': the Semantics of Action Sentences", Foundations of Language 13, 491-528

Wunderlich, $D$.

1972 "Zur Konventionalität von Sprechhandlungen", in: Wunderlich, D. (ed.) Linguistische Pragmatik (Frankfurt), 11-58

Žolkovskij, A.K.

1964 "Predislovie", Mašinnyj perevod i prikladnaja lingristika $8,3-16$

Zolkovskij, A.K./Mel'čuk, I.A.

1967 "O semantičeskom sinteze", Problemy kibernetiki $19,177-238$

1969 "K postroeniju dejstrujuščej modeli jazyka 'Smys 1

$\leftrightarrow$ Tekst'", Mašinnyj perevod i prikladnaja lingvistika $11,5-35$

1971 "Sur la synthèse sémantique", in: T.A. Informations $2 / 1970$ (Paris)

Zvegincev, V.A.

1973 Jazyk i lingvističeskaja teorija (Moskva)

1976 Predloženie $v$ ego otnošenii $k$ jazyku $i$ reči (Moskva) 
00050464 


\section{SLAVISTISCHE BEITR A GE}

105. Pogacnik, J.: Von der Dekoration zur Narration. Zur Entstehungsgeschichte der slovenischen Literatur. 1977. $165 \mathrm{~S}$.

106. Bojić, V.: Jacob Grimm und Vuk Karadzić. Ein Vergleich ihrer Sprachauffassungen und ihre Zusammenarbeit auf dem Gebiet der serbischen Grammatik. 1977. $257 \mathrm{~S}$.

107. Vintr, J.: Die ältesten Cechischen Evangeliare. Edition, Textund Sprachanalyse der ersten Redaktion. 1977. $367 \mathrm{~S}$.

108. Lohff, U. M.: Die Bildlichkeit in den Romanen Ivan AleksandroviC Goncarovs (1812-1891). 1977. XVI, $244 \mathrm{~S}$.

109. Regier, Ph. R.: A Learner's Guide to the Old Church Slavic Language. Part 1: Grammar with Exercises. 1977. XLIV, 368 S.

110. Worth, D. S.: On the Structure and History of Russian. Selected Essays. With a Preface by Henrik Birnbaum. 1977. X, $276 \mathrm{~s}$.

111. Schulte, B.: Untersuchungen zur poetischen Struktur der Lyrik von Sima Pandurović. Posmrtne pocasti.1977. $345 \mathrm{~S}$.

112. Albert, H.: Zur Metaphorik in den Epen Zivana, Medvjed Brundo, Utva und Ahasver des kroatischen Dichters Vladimir Nazor. 1977. $171 \mathrm{~s}$.

113. Slavistische Linguistik 1976. Referate des II. Konstanzer Slavistischen Arbeitstreffens $(5 .-7.10 .1976)$. Herausgegeben von W. Girke und H. Jachnow. 1977. 261 S.

114. Matuschek,H.: Einwortlexeme und Wortgruppenlexeme in der technischen Terminologie des Polnischen. 1977. VIII, $417 \mathrm{~s}$.

115. Schreier, H.: Gogol's religiöses Weltbild und sein literarisches Werk. Zur Antagonie zwischen Kunst und Tendenz. 1977. $123 \mathrm{~s}$.

116. Beiträge und Skizzen zum Werk Ivan Turgenevs. 1977. $142 \mathrm{~S}$.

117. Neureiter, F.: Geschichte der kaschubischen Literatur. Versuch einer zusammenfassenden Darstellung. 1978. $281 \mathrm{~S}$.

118. Russel, M.: Untersuchungen zur Theorie und Praxis der Typisierung bei I. A. Goncarov. 1978. $401 \mathrm{~S}$.

119. Referate und Beiträge zum VIII. Internationalen slavistenkongreB Zagreb 1978. 1978. $451 \mathrm{~S}$.

120. Slavistische Linguistik 1977. Referate des III. Konstanzer Slavistischen Arbeitstreffens Bochum. 27.9.77 - 29.9.77. Herausgegeben von W. Girke und H. Jachnow. 1978. $260 \mathrm{~S}$.

121. Müller, V.: Der Poetismus. Das Programm und die Hauptverfahren der tschechischen literarischen Avantgarde der zwanziger Jahre. 1978. VI, 215 S.

122. Pailer, $W .:$ Die frühen Dramen $M$. Gor'kijs in ihrem Verhältnis zum dramatischen Schaffen A. P. Cechovs. 1978. VIII, 210 S.

123. Thomas, G.: Middle Low German Loanwords in Russian. 1978. 269 S.

124. Lehfeldt, w.: Formenbildung des russischen Verbs. Versuch einer analytisch-synthetisch-funktionellen Beschreibung der Präsens- und der Präteritumflexion. 1978. $114 \mathrm{~S}$.

125. Schön, L.: Die dichterische Symbolik V. M. Garšins. 1978. VI, $203 \mathrm{~S}$.

126. Berg, R.: Die Abstrakta auf -nie/-tie, -ka/-ok, -ost', -stvo/ -stvie, -ie/-be in den "Pis'ma 1 Bumagi" Peters des GroBen. 1978. IV, $352 \mathrm{~S}$.

127. Stricker, G.: Stilistische und verbalsyntaktische Untersuchungen zum Moskovitischen Prunkstil des 16. Jahrhunderts. 1979. XIV, 678 S., 3 Tabellen. 
128. Heim, M. H.: The Russian Journey of xarel Havlícek Borovský. 1979. XII, 194 S.

129. Malingoudis, J.: Die Handwerkerbezeichnungen im Alttschechischen. 1979. IV, $221 \mathrm{~S}$.

130. Roth, J.: Die indirekten Erlebnisformen im Bulgarischen. Eine Untersuchung $z u$ ihrem Gebrauch in der Umgangssprache. 1979. VIII, $186 \mathrm{~s}$.

131. Nitsch, E.: Thema und Anweisungsstruktur im Text. Mit einer Analyse des ersten Abschnittes aus "Noc s Hamletem" von Viadimir Holan. 1979. VIII, $178 \mathrm{~S}$.

132. Höck, Ch.: Zur syntaktischen und kommunikativen struktur slavischer Partizipial- und Gerundialkonstruktionen. 1979. X, $283 \mathrm{~S}$.

133. Slavistische Linguistik 1978. Referate des IV. Konstanzer Slavistischen Arbeitstreffens Tübingen 26.-29. Sept. 1978 . Herausgegeben von Jochen Raecke und Christian Sappok. 1979. 276 S.

134. Breitschuh, w.: Die Feoptija V. K. Trediakovskijs. Ein physikotheologisches Lehrgedicht im RuBland des 18. Jahrhunderts. 1979..

135. Gallant, J.: Russian Verbal Prefixation and Semantic Features : an Analysis of the Prefix B3-. 1979.460 S.

136. Jachnow, H. (u.a.): Zur Erklärung und Modellierung diachroner Wortbildungsprozesse (anhand russischer substantivischer Neologismen). 1980. IV, $230 \mathrm{~S}$.

137. Breu, W.: Semantische Untersuchungen zum Verbalaspekt im Russischen. 1980. X, $231 \mathrm{~S}$.

138. Slavistische Linguistik 1979. Referate des V. Konstanzer Slavi-. stischen Arbeitstreffens Zürich 25. - 27. Sept. 1979. Herausije-. geben von Daniel Weiss. 1980. $259 \mathrm{~S}$.

139. Franz, N.: Groteske Strukturen in der Prosa Zamjatins. Syntaktische, semantische und pragmatische Aspekte. 1980. $312 \mathrm{~S}$.

140. Baer, J. T.: Arthur Schopenhauer und die russische Literatur dess späten 19. und frühen 20. Jahrhunderts. 1980. VIII, $194 \mathrm{~S}$.

141. Lempp, A.: Das zusammengesetzte Verbalprädikat mit "da" im Neubulgarischen. 1981. IV, $102 \mathrm{~S}$.

142. Stephan, H.: "Lef" and the Left Front of the Arts. 1981. XIV, $242 \mathrm{~S}$.

143. Kempgen, S.: "Wortarten" als klassifikatorisches Problem der deskriptiven Grammatik. Historische und systematische Untersuchungen am Beispiel des Russischen. 1981. X, $309 \mathrm{~S}$.

144. Peters, J.: Farbe und Licht. Symbolik bei Aleksandr Blok. 1981. VIII, $315 \mathrm{~s}$.

145. Ebding, J.: Tendenzen der Entwicklung des sowjetischen satirischen Romans (1919-1931). 1981. VI, $294 \mathrm{~S}$.

146. Schreiber, J.: Jerzy Andrzejewskis Roman "Ciemnosci kryja zieniie" und die Darstellung der Spanischen Inquisition in Werken der fiktionalen Literatur. 1981. VIII, $308 \mathrm{~s}$.

147. Slavistische Linguistik 1980. Referate des VI. Konstanzer Slayilstischen Arbeitstreffens Hamburg 23. - 25. Sept. 1980. Herausie?geben von Peter Hill und Volkmar Lehmann. 1981. $243 \mathrm{~S}$.

148. Hartenste in, K.: Das erklärend-kombinatorische worterbuch in "Smysl $\rightarrow$ Tekst"-Modell. Studien zu den lexikologischen Grurid al $_{1}-$ gen der Bedeutungsexplikation und ihrer lexikographischen V6rwendbarkeit. 1981. VIII, 277s

\begin{tabular}{|c|c|}
\hline $\begin{array}{l}\text { Baye:lsche } \\
\text { Staatsbibllothek } \\
\text { München }\end{array}$ & $\begin{array}{r}\text { Klaus Hartenstein - 9783954792764 } \\
\text { ownloaded from PubFactory at 01/10/2019 05:16:44AM } \\
\text { via free access }\end{array}$ \\
\hline
\end{tabular}

